\title{
Beyond the tension of hypertension : focus on non- antihypertensive aspects of antihypertensive treatment
}

Citation for published version (APA):

van Bortel, L. M. A. B. (1993). Beyond the tension of hypertension : focus on non-antihypertensive aspects of antihypertensive treatment. [Doctoral Thesis, Maastricht University]. Rijksuniversiteit Limburg. https://doi.org/10.26481/dis.19931118lb

Document status and date:

Published: 01/01/1993

DOI:

10.26481/dis.19931118lb

Document Version:

Publisher's PDF, also known as Version of record

Please check the document version of this publication:

- A submitted manuscript is the version of the article upon submission and before peer-review. There can be important differences between the submitted version and the official published version of record.

People interested in the research are advised to contact the author for the final version of the publication, or visit the DOI to the publisher's website.

- The final author version and the galley proof are versions of the publication after peer review.

- The final published version features the final layout of the paper including the volume, issue and page numbers.

Link to publication

\footnotetext{
General rights rights.

- You may freely distribute the URL identifying the publication in the public portal. please follow below link for the End User Agreement:

www.umlib.nl/taverne-license

Take down policy

If you believe that this document breaches copyright please contact us at:

repository@maastrichtuniversity.nl

providing details and we will investigate your claim.
}

Copyright and moral rights for the publications made accessible in the public portal are retained by the authors and/or other copyright owners and it is a condition of accessing publications that users recognise and abide by the legal requirements associated with these

- Users may download and print one copy of any publication from the public portal for the purpose of private study or research.

- You may not further distribute the material or use it for any profit-making activity or commercial gain

If the publication is distributed under the terms of Article $25 \mathrm{fa}$ of the Dutch Copyright Act, indicated by the "Taverne" license above, 


\section{BEYOND THE TENSION OF HYPERTENSION}

focus on non-antihypertensive aspects of antihypertensive treatment 


 क्र}

\section{CIP-DATA KONINKLJJKE BIBLIOTHEEK, DEN HAAG}

Bortel, Lucas Maria Aloysius Barbara Van

Beyond the tension of hypertension: focus on non-antihypertensive aspects of antihypertensive treatment / Lucas Maria Alyosius Barbara Van Bortel. [S.I. : s.n.] Thesis Maastricht. - With ref. - With a summary in Dutch. ISBN 90-9006590-3

NUGI 741

Subject headings: hypertension / quality of life / artery compliance.

Publication of this thesis was supported by the Janssen Research Foundation. 


\section{BEYOND THE TENSION OF HYPERTENSION}

\section{focus on non-antihypertensive aspects of antihypertensive treatment}

\section{Proefschrift}

ter verkrijging van de graad van doctor aan de Rijksuniversiteit Limburg te Maastricht, op gezag van de Rector Magnificus, Prof. dr. H. Philipsen, volgens het besluit van het College van Dekanen, in het openbaar te verdedigen op donderdag, 18 november 1993 om 14.00 uur

\section{door}

Lucas Maria Aloysius Barbara Van Bortel geboren te Kalmthout in 1949 
Beoordelingscommissic Prof. dr. R.S. Reneman, voorzitter Prof. dr. J. van Ree

Prof. dr. M. Safar, Universite P\&M Curie, Paris Prof. dr. Th. Thien, Katholicke Universiteit, Nijmegen Prof. dr. J. Urquhart 
$\pi \alpha \nu \tau \alpha, \rho \epsilon \iota$ (alles verandert; Herakleitos, $\pm 500 \mathrm{~A} . \mathrm{C}$.)

aan mijn ouders

aan Marleen, Tine, Jeroen en Stijn 
the

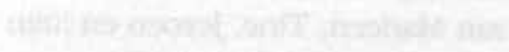




\section{CONTENTS}

\section{PART I}

\section{INTRODUCTION}

chapter 1. pathophysiology and epidemiology of hypertension I1

1. blood pressure and blood pressure control II

2. epidemiology of hypertension 12

3. ill effects of hypertension $\quad 13$

chapter 2. functional and structural changes of the cardionascular system in hypertension and their influence on morbidity and mortality 14

1. changes resulting in an increased mean arterial pressure 14

2. changes resulting in an increased pulse pressure $\quad 15$

3. left ventricular hypertroplyy $\quad 15$

3.1. cardiac remodeling in hypertension $\quad 15$

3.2. effect of cardiac hypertrophy on heart function $\quad 16$

3.3. risk of cardiac bypertrophy - 16

4. (coronary) atherosclerotic disease $\quad 17$

4.1. Onset of atheroselerosis $\quad 17$

4.2. progression to atherosclerotic disease _......... 18

5. stroke ................................ 19

6. renal failure $\quad 20$

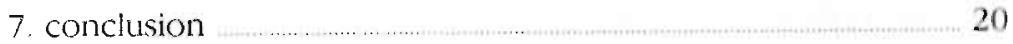

chapter 3. management of hypertension $\quad 21$

1. reduction of high blood pressure $\quad 21$

1.1. nonpharmacological management _......... 21

1.2. pharmacological management _..... 22 .

1.2.1. history ...................... 22

1.2.2. most important drugs currently used _._._._._... 23

1.2.3. results of pharmacological treatment of
hypertension

1.2.4. drug adherence

2. prevention of ill effects of hypertension $\quad 26$

2.1. treatment and prevention of cardiac hypertrophy $\quad 26$

2.2. prevention of ischacmic cardiovascular events $\quad 27$

2.2.1. prevention of atherogenesis _...27

2.2.2. prevention of plaque rupture _......... 28

2.2.3. prevention of thrombosis and vasospasm _........... 28

2.2.4. influence on risk factors for atherosclesosis _....... 29

2.3. prevention of stroke _... 31

2.4. prevention of renal failure _..._. 31

this thesis $\quad 33$ 
PART II

INFIUENCE OF PHYSICAI. FACTORS ON THE VESSEL WALL

chapter 1. physical factors on large arteries and antihypertensive therapy

1. pulsatile stress in the arterial wall $\quad . . . .35$

1.1. vessel wall propertics of large arteries _....... 36

1.2. pulse wave reflections $\quad 37$

1.3. other determinants of pulsatile stress ... $\quad 37$

2. shear stress $\quad 38$

5. conclusion $\quad 39$

chapter 2. the effect of verapamil on carotid artery distensibility and cross-sectional compliance in hypertensive patients 40

chapter 3. effect of nebivolol on distensibility and compliance of the common carotid artery

PAR'T III

QI ALITYY OT LIFE: PERCEPTION DIIRING

ANTIIIYPLRTLNSIVI: TREATMENT

introduction

chapter 1. effect of placebo and antihypertensive treatment with nebivolol on hamodynamics and quality of life measured with the Inventory of Subjective Health.

chapter 2. 1)o ACE-inhibitors preserve quality of life better than other antihypertensive drugs? A comparative study between cnalapril and bisoprolol

chapter 3. exercise tolerance during antilypertensive treatment 
PART IV

GENERAL DISCUSSION : CURRENT ANTIHYPERTENSIVE THERAPY

AND ISSUES FOR FURTHER RESEARCII

1. which blood pressure to treat?

2. how far should blood pressure be lowered?

3. what antihypertensive drug to choose?

3.1. rationale 88

3.1. I. effect on risk factors as discriminating criterion? 88

3.1.2. major criteria to make a choice 89

3.2. effect of antihypertensive drugs on criteria for drug choice 90

3.2.1. diuretics 90

3.2.2. ACE-inhibitors 90

3.2.3. calcium-antagonists 91

3.2.4. $\alpha$-blockers 92

3.2.5. B-blockers 92

3.3. influence of concomitant diseases or conditions

on the choice of antihypertensive drugs 93

4. monotherapy or low-dose combination therapy?

5. a personal view on current treatment of hypertension 94

6. individualizing therapy 95

7. issues for future research $\quad 95$

7.1. evaluation of current antihypertensive therapy _..... 95

7.2. prevention of ill effects ......................... 95

7.3. reversal of ill effects $\quad 96$

7.4. around the clock protection $\quad 96$

7.5. identification of patients at risk $\quad 97$

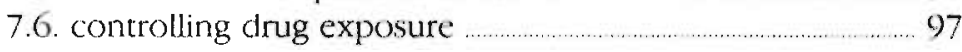

ACKNOWLEDGEMENTS $\quad 99$

REFERENCES

101

SUMMARY

SAMLNVATTING

131

CLIRRICIILUM VITAE 


\section{PART I \\ INTRODUCTION}

\section{chapter 1. pathophysiology and epidemiology of hypertension}

\section{1. blood pressure and blood pressure control}

From a physiological point of view, systolic and diastolic blood pressure are determined by the mean arterial pressure (MAP) and the pulse pressure ( $\Delta P$ ) oscillating around this mean arterial pressure. Determinants of blood pressure are shown in Table 1.1.

Table 1.1. Determinants of blood pressure

\begin{tabular}{|l|l|l|}
\hline components & mean arterial pressurc & pulse pressure \\
\hline nature & static & dynamic \\
\hline $\begin{array}{c}\text { main determinant vessels } \\
\text { resistance vessels : } \\
- \text { small arteries } \\
\text { - arterioles } \\
\text { - capillaries }\end{array}$ & $\begin{array}{c}\text { capacitance vessels: } \\
- \text { small arteries } \\
\text { - large arteries }\end{array}$ \\
\hline
\end{tabular}

Mean arterial pressure - the static component of blood pressure - is determined by cardiac output (CO) and systemic vascular resistance (SVR): MAP $=$ CO $x$ SVR. The cardiac output is determined by the heart rate (HR) and stroke volume (SV): $\mathrm{CO}=\mathrm{HR} \times \mathrm{SV}$

The systemic vascular resistance is a function of blood viscosity $(\eta)$ and the resistance determined by small resistance vessels, which are predominantly arterioles and capillaries. This is described in more detail in chapter 2.

For a given stroke volume (SV), pulse pressure - the dynamic part of the blood pressure - is determined by the compliance (C) of arterial capacitance vessels, which are predominantly large arteries (1):
$\Delta \mathrm{P}=\mathrm{SV} / \mathrm{C}$

Compliance is a quantitative measure of the buffering capacity of vessels. However, this formula of $\Delta \mathrm{P}$ is incomplete since, apart from compliance of large vessels, not only stroke volume, but also the velocity of ejection of the blood from the heart and reflected pulse waves can influence pulse pressure [see part II chapter 1] (1).

In summary: systolic and diastolic blood pressure are a result of the cardiac pump function and the static and dynamic resistance (impedance) of the arterial tree. For a given heart function, arterioles and capillaries largely account for the static component of the blood pressure (MAP), while large arteries determine predominantly the dynamic part $(\Delta P)(1)$.

Short-term variability (over a 30 -minute period) in arterial pressure of adult man is smaller than $10 \%$ of the mean pressure. This is due to blood pressure control systems. The body has many systems for controlling the pressure, which has been extensively described $(4,5)$. Examples of them are shown in Table 1.2. 
Table 1.2. Examples of blood pressure comtrollers (4)

\begin{tabular}{|l|l|l|}
\hline short-term & intermediate & long-term \\
\hline baroreceptor reflex & renin-angiotensin system & kidney-fluid control \\
\hline CNS ischaemic reflex & stress relaxation & \\
\hline chemorceeptor reflex & capilary fluid shift & \\
\hline & aldestetone & \\
\hline
\end{tabular}

Controllers based on the nervous system act within seconds and are thus shortterm controllers. Intermediate pressure controllers function a few minutes after activation of the neural pressure controllers $(6,4)$. Long-term control is achieved by the kidney-fluid system, which takes usually 2 to 4 days before pressure equilibrium has been reaclicel (4).

\section{2. epidemiology of hypertension}

Hypertension, defined as a resting diastolic blood pressure over $95 \mathrm{mmHg}$ is a very common condition in westernised countries. 15 to $20 \%$ of American adults, as evidenced by a National Health Survey, have hypertension ( 7 ); if hypertension is defined from a level of more than $90 \mathrm{mmHg}$, about one third of the population is affected (8). About $1 \%$ of hypertensive patients develop malignant hypertension (9), characterixed by an extremely high blood pressure accompanied by neuroretinopathy with papillocelema and exudates with haemorrhages (10).

Hypertension can be secondary to different diseases and may be drug related (11). Examples of secondary hypertension are shown in Table 1.3. Ferguson reported that secondary hypertension was most frequently oral anticonceptive-induced $(40 \%) ; 30 \%$ of secondary hypertension was due to renovascular disease, about $20 \%$ due to chronic renal disease and about $2 \%$ due to Conn's syndrome (12). In 90 to $95 \%$ of cases the actiology of hypertension is still largely unknown $(9,12)$. This means that $>90 \%$ suffer from primary or essential hypertension.

Table 1.3. Some examples of secondary bypertension

\begin{tabular}{|l|l|l|l|}
\hline renal discase & adrenal discasc & drugs & other \\
\hline glomerulonephritis & Cishing's syndrome & contraceptive pill & pre-eclampsia \\
\hline polycystic disease & Conn's syndrome & liquorice & coarctation of aorta \\
\hline pyelonephritis & phacochromocytoma & NAO-inhibitors & $\begin{array}{l}\text { raised intracranial } \\
\text { tension }\end{array}$ \\
\hline renovascular disease & & corticoids & \\
\hline renin secreting itumor & & sympathomimetics & \\
\hline
\end{tabular}

Essential hyperiension is a multifactorial disease (13). Genetic as well as environmental factors and excessive psychological stress - can increase the risk for essential hypertension (14-16). High blood pressure before the age of 55 occurs 3.8 times more often among persons with a strong positive family history of high blood pressure (17). Williams believes that most familiat aggregations of high blood pressure is due to genetic rather than due to shared familial environment (18), with 
about $12 \%$ of hypertensives suffering from familial dyslipidaemic hypertension. Different environmental risk factors relate to modern lifestyle, i.e. high salt intake, high dietary sodium/potassium ratio, caloric imbalance with resultant obesity, and high alcohol intake (19). An overview of published observations suggests that both genetic predisposition and environment work together to produce hypertension in most persons (18). For example, hyperinsulinaemia and insulin-resistance might be a genetic and/or environmental important risk factor for hypertension. The linkage between obesity and hyperinsulinaemia due to insulin resistance is well known (20). Hyperinsulinaemia could lead to hypertension through stimulation of 4 intermediate factors: renal sodium reabsorption and sodium retention (21). plasma norepinephrine concentration, vascular hypertrophy and intracellular calcium concentration (22). In addition, Reaven described a syndrome $X$, characterized by [1] resistance to insulin-stimulated glucose uptake, [2] ghucose intolerance, [3] hyperinsulinaemia, [4] increased concentrations of very low density lipoprotein triglyceride, $[5]$ decreased concentration of high density lipoprotein cholesterol and [6] hypertension (23). Reaven's syndrome X is thought to be linked with an increased risk for coronary artery disease. In family studies it occurred in 12 percent of all persons with essential hypertension, but in $25 \%$ of those with hypertension beginning before the age of 60 (24). Syndrome $X$ can also exist in persons who are otherwise thought as healthy (25). Reaven's syndrome $X$ should be distinguished from another recently described Syndrome $X$, also of unknown aetiology, which includes typical angina, a positive exercise test, a normal coronary angiogram, and a normal resting cardiac function but a reduced coronary vasodilatory capacity (26).

\section{Ill effects of hypertension}

Malignant hypertension has a grave prognosis: $50 \%$ of untreated parients die within 3 months and very few survive more than 1 ycar (10). Death is usually due to renal failure, often accompanied by heart failure and stroke. Cumulative death from malignant hypertension is positively related to the height of the blood pressure (27). Due to active treatment the morbidity and mortality risk of hypertension has been changed from malignant hypertension to hypertension-induced progressive atherosclerosis and its chief sequelac (28). The major target organs of hypertension are the blood vessels, heart, brain and kidneys (29). Major ill effects of hypertension are stroke, those due to cardiac hypertrophy and atherosclerosis - coronary atherosclerosis in particular - and to a lesser extent renal failure (30). In the Framingham study the annual incidence of coronary disease was $306 / 10,000$ in hypertensive men versus $66 / 10,000$ for stroke (31). Thus, the risk of coronary heart discase was 5 times more frequent than the risk of stroke. 


\section{chapter 2. functional and structural changes of the cardiovascular system in hypertension and their influence on morbidity and mortality}

\section{1. changes resulting in an increased mean arterial pressure}

In most cases of essential hypertension mean arterial pressure is increased. Mean arterial pressure is determined by cardiac output and peripheral resistance. In early hypertension, an increase in cardiac output might be the reason for the elevated blood pressure (15) with or without an increase in peripheral resistance. In established hypertension peripheral resistance is nearly always increased. This increase in peripheral resistance plays a key role in the onset and maintenance of essential hypertension. The factors that determine vascular resistance are the number of blood vessels $[N]$, the length of the vessels [L], the blood viscosity [ $\eta$ ], and the raditis of the vessels $[\mathrm{r} \mid$. The quantitative relationship between these variables and total resistance $(\mathrm{R})$ is given by Poiscuille's law:

formula: $\mathrm{R}=8 \eta \mathrm{L} / \mathrm{N} \pi \mathrm{r}^{-1}$

Although this is a relatively simple formula to describe, the actual physiology of resistance control and changes in hypertension is complex. This complexity is caused by the architecture of the vascular system, its heterogeneity, both within a given vascular bed and berween different vascular beds, and the many factors influencing vascular resistance (Table 1.4).

Three mechanisms have been proposed to underlic the overall vascular resistance increase in hypertension: [a] rarefaction of arterioles and capillaries; [b] decreased internal diameter of the arterioles and small arteries; and $[\mathrm{c}]$ increase in arterial and arteriolar wall mass (32-36).

Traditionally, the decrease in internal diameter was regarded as the most important cause of resistance increase in hypertension. Modern views regard internal diameter as an important short-term controller of vascular resistance, whereas the number of small vessels and vascular mass are important in the long-term control of vascular resistance $(32-33,35-38)$.

Table 1.4. Factors influencing vascular resistance

\begin{tabular}{|c|c|c|}
\hline blood viscosity & smooth muscle activity & structural factors \\
\hline hacmatocrit & myogenic response & $\begin{array}{l}\text { wall/lumen ratio: } \\
\text {-structural diameter } \\
\text {-wall thickness } \\
\text {-distensibility }\end{array}$ \\
\hline velocity & electrolytes & $\begin{array}{l}\text { network characteristics: } \\
\text {-number } \\
\text {-length } \\
\text {-branching pattern }\end{array}$ \\
\hline cell aggregation & nerves & \\
\hline cell deformation & $\begin{array}{l}\text { metabolites } \\
\text { endothetium-derived substances }\end{array}$ & \\
\hline
\end{tabular}

I Part of this ctupter is based on: Struyker Boudier HAJ. Van Bortel LMAB, De Mey JGR. Remodeling of the vascular tree in hypertension: drug effects. Trends Pharmacol Sci 1990, 11: 240-245. 
Microvascular rarefaction can be observed as a feature of several tissues in carly stages of development of hypertension. The nature of the mediators of microvascular rarefaction in hypertension is still unknown. One possibility is that it represents an adaptive response to increased microvascular pressure or flow $(33,39)$. Alternatively, rarefaction may be the result of a decreased capacity of the hypertensive animal to form new blood vessels (angiogenesis).

Folkow has proposed that an increase in contractile mass is an important feature of the arterial and arteriolar vessel wall in hypertension (32). Increased vessel wall mass is part of a general process to remodel blood vessels in response to a variety of stimuli. The mediators of the increase in vessel wall mass are still largely unknown. However, there is increasing evidence that the endothelum plays a crucial role in controlling growth in the vessel wall. By sensing changes in local haemodynamics and by acting as an important source of molecules affecting growth, the endothelium is a unique controller of both vascular smooth muscle tone and vessel wall structure $(40)$.

\section{2. changes resulting in an increased pulse pressure}

Ventricular ejection and large artery visco-elastic properties influence the level of pulse pressure (1). In essential hypertension, the increase in pulse pressure is predominantly due to stiffening of the large arteries. This is described in detail in part II, chapter 1. Different factors may contribute to the stiffening of large arteries, for example, a decreased connective tissue elasticity, atherosclerosis and a decrease in smooth muscle relaxation (41).

Systolic blood pressure is more closely related to pulse pressure, while diastolic pressure better reflects mean arterial pressure. The Framingham study and also other epidemiological studies have shown that the ill effects of hypertension are better correlated with the systolic than with the diastolic blood pressure (42-44), indicating an important role of the pulsatile stress (pulse pressure).

Increased pulse pressure is most pronounced in elderly hypertensive patients. It results in an increase in systolic pressure, while diastolic pressure tends to decrease in relation to the mean arterial pressure (1). Apart from combined systolodiastolic hypertension, isolated systolic hypertension (ISH) occurs relatively often in elderly patients. The prevalence of ISH increases with age. It ranges from $7 \%$ in individuals aged 60 to 69 years to more than $20 \%$ in those aged 80 years and more (45-46). In isolated systolic hypertension mean arterial pressure and vascular resistance may be normal or only slightly elevated, while pulse pressure is increased (1).

\section{3. left ventricular hypertrophy}

Left ventricular hypertrophy, as detected by echocardiography, is present in approximately $20-50 \%$ of hypertensive patients ( 47 ).

\section{1. cardiac remodeling in hypertension}

Mechanical overload increases wall stress of the left ventricle, which is considered the main pathogenic stimulus for cardiac hypertrophy (48). The true load for the heart is the impedance of the ascending aorta, being to the pulsatile pressure and flow what resistance is to continuous flow. In hypertensive heart disease, it depends on the arteriolar resistance, compliance of the large vessels (predominanty 
the: aorta), blood inertia and also on the reflected pressure waves (49). Thus, the compliance of large arteries plays an important role both in the onset and maintenance of cardiac hypertrophy in hypertension (50-51). The importance of mechanical factors is discussed in more detail in part II.

Mechanical overload is known to influence several (neuro)hormonal systems, including the sympathetic nervous system and the renin-angiotensinaldosterone system. Overactivity of these systems seems to be important in driving the early asymptomatic remodeling process (52). Apart from the sympathetic nervous system and the renin-angiotensin-aldosterone system, other undefined cytokines and peptides afe probably also involved (53). Cardiac hypertrophy is characterized by quantivite and qualitative changes in the myocardium and coronary arterioles: [1] myocyte hypertrophy; [2] slowing the maximal shortening velocity $\left(V_{\max }\right)$ of the myocytes due 10 an increased proportion of the 18 isoform of myosin, which can totally replace the $\alpha$ isoform (54); [3] possibly alteted calcium homeostasis in the myoeyte; $\mid$ f hypertension-induced coronary artery hypertrophy resulting in a smalker compliance and higher coronary resistance; [5] increase in collagen content in the interstitium of the heart (55). Collagen may be produced in response to fibroblast stretch (56), and by activation of trophic factors including angiotensin II and aldasterone (57), in patients with hypertension, left ventricular mass is strongly correlated with plasma aldosterone levels (58). Recently, a role of aldosterone in the development of left ventricular fibrosis, associated with left ventricular hypertrophy, has been suggested (59). These findings suggest that the increased afterload for the heart in hypertension is the drive for (reversible) cardiac hypertrophy, while the elevated angiotensin II and aldosterone levels not only maintain hypertension by salt and water retention, but further impair cardiac function by (hardly reversible) tibrosis.

\section{2. effect of cardiac hypertrophy on heart function.}

Diastolic function is abnormal in hypertension. This abnomality is seen at a stage before left ventricular hypertrophy is obvious $(60)$. It reflects chamber stiffness or relaxation abnormalities (61). Systolic function is, in general, nomal in hypertensive patients with left ventricular hypertrophy $(61)$. However, with progressive hypertrophy, systolic function may become impaired and may result in an initially asymptomatic state with decreased ejection fraction, which can further deteriorate to oven heart failure (62).

\section{3. risk of cardiac hypertrophy}

In bypertension, an increase in left ventricular mass provides the best prediction of (fatal and nonfatal) cardiovascular clinical events (63). Possible reasons why left yentricular hypertrophy might be such a lethal condition are: cardiac hypertrophy leads to [1] a mismatch between blood supply and non-vascular tissue resulting in a relatively 'starved' subendocardial region; [2] an increased basal myocardial oxygen demand due to increased mass and wall stress; [3] a markedly reduced coronary flow reserve (ability to clilate coronary aneries) making ischacmia more likely under stressful conditions (64); [ 4 ] an increase in ventricular arrhythmias and sudden death (65-66). This might be due to an excess of fibrous tissue, interference with coronary flow or disturbances in the ventricular pump function irself $(62,64)$. The association of left ventricular hypertrophy and arrhythmia might also be due 
to (impending) heart failure. This is supported by the observation that, in patients with heart failure, antihypertensive treatment with the ACE-inhibitor enalapril reduced mortality especially from progressive heart failure, while there was no effect on mortality from arrhythmia without previous worsening congestive heart failure $(67-68)$.

In conclusion, cardiac hypertrophy is common in hypertension and is a lethal condition. Therefore, measurement of left ventricular mass might be a better tool for the evaluation of antihypertensive treatment.

\section{4. (coronary) atherosclerotic disease}

\subsection{Onset of atherosclerosis.}

Vascular injury is a key event in the response-to-injury hypothesis of atherogenesis (69). The definition of endothelial injury has been problematic, since there may be a spectrum of changes in injured endothelium and many of these may have no morphologic manifestations. Fuster proposed 3 types of vascular injuries (Table 1.5), which might be involved in onset and progression of atherosclerosis ( 70 ).

\section{Table 1.5. Types of endotbelial injury}

\begin{tabular}{|l|l|}
\hline type & nature \\
\hline type I & functional alterations of endothelial cells without morphologic changes \\
\hline type II & endothelial denudation and intimal damage with intact internal clastic lamina \\
\hline type III & endothelial denudation with damage of both intima and nedia \\
\hline
\end{tabular}

Endothelial injury (type I and II) may result in release of growth factors from one or more of the following cells : the endothelium, monocytes and platelets. Growth factors induce smooth-muscle cell migration and proliferation and possibly autogenous growth factor release by stimulated smooth-muscle cells.

All four principal cells involved in atherosclerosis - endothelium, smooth muscle cells, platelets, and monocyte/macrophages - cither contain (c.g., platelets) or can synthesize and release chemo-attractants and growth factors (69). These interactions could then lead to fibrous-plaque formation and further lesion progression. In spontancous atherosclerosis, the tenet is that chronic minimal injury to the arterial endothelium is caused mainly by a disturbance in the pattern of blood flow in certain parts of the arterial tree, especially at bending points and areas near branching vessels $(69,71-72)$. The influence of physical factors is discussed below. In experiments in animals, chronic mild endothelial injury may also be potentiated by hypercholesterolaemia, circulating vasoactive amines, immunocomplexes, infection, and chemical irritants in tobacco smoke (73).

Atherosclerosis is of multifactorial origin. Its genesis is influenced by dyslipoproteinaemia, thrombotic events, haemodynamic/mechanical and endogenous chemical injury of the arterial intima (or media), as well as inflammatory and/or immune reactions of the arterial wall (74). Risk factors for atherosclerosis are: hypertension, cigarette smoking, glucose intolerance, insulin resistance, obesity, hypercholesterolaemia, hypertriglyceridaemia [?], and personality type (31). 
Table 1.6. Possible biologic mecbanisms of risk faciors in atberogenesis.

\begin{tabular}{|c|c|c|c|c|c|c|}
\hline & Hi. & IR & $\mathrm{HT}$ & SM & ES & GE \\
\hline vascular injury (type l) & + & + & + & + & + & 一 \\
\hline lipid deporsition & ++ & ++ & - & - & - & ++ \\
\hline monexyte/platelet deposition & 4 & + & - & - & - & - \\
\hline Dibromtimal prosliferation & + & ++ & + & + & + & + \\
\hline plaçue disnoption(type [I//II) & ++ & ++ & ++ & ++ & ++ & - \\
\hline Thrombosis & + & + & - & ++ & + & + \\
\hline
\end{tabular}

Adapted from Ip ct al (73), HL: hyperlipiblacmia; IR: insulin resistance; HT: hypertension:

SM: smoking; IS: cmotional stress; GE: genetics; + mild; + + : severe; - - no effect.

Athough atherosclerosis is a separate disease that has a natural history independent of hypertension, it is exacerbated by hypertension (29). Hypertension may initiate and perpetuate vascular injury, or both, and may enhance the resultant fibrocellular proliferative responses (29). However, there is some experimental evidence that hypertension by itself may not be a necessary or adequate atherogenic factor (75). But more importanty, hypertension can play a role in triggering acute plaque rupture caused by physical stress on the arterial wall.

\section{2. progression to atherosclerotic disease.}

In most growing lesions progression is probably rapid. Energing evidence from autopsy studies $(70)$ suggests that it follows recurrent minor fissures of the most fatty or atheromatous plaques with subsequent thrombus formation and fibrotic organisation. Most of the fissures probably reseal and incorporate thrombus at the same time but do not produce clinical symptoms. However, a deep plaque fissure or ulceration may lead to thrombotic occlusion and acute coronary syndrones. Increased shear forces in the area of stenosis, sudden changes in intraluminal coronary pressure or tone (77-78) and bending and twisting of an artery during each heart contraction may contribute to the disruption of these plaques. Computer modelling also showed that the distribution of circumferential tensile stress across the intima was radically altered by atherosclerotic plaques. Regions of high circumferential stress cortelated well with the site of intimal tears found at necropsy (79). Clearly, the role of mechanical or physical stresses in plaque disruption requires further intensive investigation $(70)$. In comparison with other large arteries, coronary arteries undergo an additional stress exerted by the contraction of the heant itself. It seems logical that both heart rate and contractility might influence this additional stress. This view is supported by the observation that ischaemic episades $(80)$, myocardial infarction $(81,82)$ and sudden death $(83,84)$ have a peak occurrence in the morning waking hours, which correspond closely with a peak in heart rate and blood pressure (85) and also in indices of coagulation (86-90) such as increased platelet and decreased fibrinolytic activity.

The relative prevalence of this process of plaque disruption with mural thrombus formation and organization, in comparison with the more progressive myointimal proliferative process due to chronic endothelial injury postulated by Ross is unknown. However, several groups $(76,79,91-94)$ have clearly shown that thrombus formation, usually due to atherosclerotic plaque rupture plays a fundamental 
role in the development of the acute coronary syndromes. The disrupted plaques are the so-called complicated plaques (70).

Recent evidence from a prospective angiographic study (95) suggests that severe stenoses tend to progress to total occlusion about 3 times more frequently than do less severe lesions, but this process only infrequently results in myocardial infarction. In contrast, $85 \%$ of infarct-related lesions were not hacmodynamically important (stenosis of less than $75 \%$ of the diameter). In addition, other authors have found that about two third of the lesions responsible for myocardial infaretion had a stenosis less than 50-70\% (96-99). Unlike small plaques, which may be lipid-rich and prone to disruption, severely stenotic plaques tend to be very fibrotic and stable (100). These findings support the concept that disruption of small plaques is important in the pathogenesis of acute myocardial infarction, whereas longstanding severe stenoses more commonly resuit in total vessel occlusion, with a small or silent infarction or no infarction at all, perhaps because of the presence of well-developed collateral vessels (101-103).

Although a substantial proportion of episodes of unstable angina and acute myocardial infarction is caused by plaque fissuring or rupture with superimposed thrombosis, other mechanisms that alter the balance between myocardial oxygen supply and demand also need to be considered (104). Vasospasm was found to be an important contributor to intermittent coronary artery occlusion in patients with acute myocardial infarction who were treated with streptokinase (105). Animal experiments showed that platelets contribute to a reduction in blood flow, either by transiently aggregating and occluding the vessel, by releasing vasoconstrictor substances like serotonin and thromboxane $A_{2}$ (106-109), or both (94). In addition, atherosclerotic arteries have an abnormal vasodiator function, perhaps related to a deficiency in the production of endothelium-clerived relaxing factor $(110)$.

\section{5. stroke.}

The prevalence of stroke is estimated to be $50-80$ per 10000 population over the age of 25 years (111). Stroke is the third leading cause of death and an importan cause of hospital admission and long-term disability in most industrialiscd countric's. It accounts for 10-12\% of all deaths in industrialised countries (112). In addition, the cumulative risk of recurrence over 5 years is high, ranging from about a third to almost a half of people who have stroke (112). Risk factors for stroke are shown in Table 1.7.

Table 1.7. Factors that predict future occurrence of stroke.

\begin{tabular}{|l|l|l|}
\hline physiological & behaviour & other \\
\hline blood pressure & diet & social class \\
\hline serum cholesterol & smoking & psychosociaf factors \\
\hline fibringgen & alcohol & ethnic groups \\
\hline body mass index & oral contraceptives* & age \\
\hline blood sugar & & \\
\hline
\end{tabular}

* only data on older oral contraceptives available 
In patients with blood pressure $\geqq 160 / 95 \mathrm{mmiHg}$, the relative risk of stroke was 2.7 for men and 2.3 for women compared with normotensive men and women (113). Also in isolated systolic hypertension the risk of stroke was increased at least twofold (114). In addition, about $88 \%$ of the deaths attributed to stroke are among people over 65 years (112). Stroke incidence rises exponentially with increasing age, with a hundred-fold increase in rates from about 3 per 10000 population per year in the third and fourth decades to almost 300 in the eight and ninth decades (115). There are 3 main classes of stroke: ischaemic stroke, intracerebral haemorrhage and subarachnoid hacmorrhage (116). Raised serum cholesterol is strongly related to death from non-haemorrhagic stroke, but not from intracerebral and subarachnoid haemorrhage. The major risk factor for stroke, whether haemorrhagic or not is high blood pressure (1 16).

\section{6. renal failure.}

Renal dysfunction is almost always demonstrable in hypertensive patients, even in those with minimally elevated pressures. This often asymptomatic involvement may be detected by increased microalbuminuria, reflecting intraglomentar hypertension (117). It results from an increased preglomerular arteriolar flow and decreased resistance. This leads to glomerular hypertension, glomerular hyperper fusion and glomerular injury and sclerosis, referred to as nephrosclerosis (1 18-119). The loss of renal function is positively related to the blood pressure (120), but only a minority of hypertensives develop terminal renal failure. Renal failure represents either the effects of the malignant phase of hypertension caused by fibrinoid arterial necrosis or the progression of some primary renal disease unidentified in the search for a specific cause. Very rarely the kidneys may fail due to other forms of arterial disease, particularly atheroma of the larger vessels and nephrosclerosis (121). The elevated serum uric acid level present in one-third of untreated hypertensives possibly reflects nephrosclerosis (122). Hypertension accounts for 20 to $25 \%$ of all causes of renal failure in the United States (123). The relative risk for hypertensive end-stage renal disease is about $5 \%(124)$. It is more common in blacks than in caucasians.

\section{7. conclusion}

Except for isolated systolic hypertension, the onset and maintenance of hypertension is mainly based on functional and structural changes in arterioles and capillaries. The ill effects are not only due to the elevated pressure (i.e. stroke), but even more importantly to acceleration of atherosclerosis and cardiac hypertrophy. In conclusion, the onset and maintenance of hypertension is predominantly due to changes in the microcirculation, while the majority of ill effects are due to pathological changes in the macrocirculation.

In isolated systolic hypertension both onset and ill effects are predominantly due to changes in the macrocirculation. 


\section{chapter 3. management of hypertension}

\section{1. reduction of high blood pressure \\ 1.1. nonpharmacological management}

Various nonpharmacological therapies are being widely advocated as initial therapy - at least for the first 3-6 months after recognition of hypertension - for most patients with a slightly elevated blood pressure (125-126). Different nondrug therapies have been proposed. Recently, their long-term effectiveness has been reviewed by Kaplan (127) and is shown in Table 1.8.

Table 1.8. Long-term effectiveness of proposed nonpbarmacological treatment.

\begin{tabular}{|l|l|l|}
\hline effective & minimat, if any & no evidence at all \\
\hline overweight reduction & potassium & coffec reduction \\
\hline salt restriction & magnesium & smoking reduction \\
\hline physical exercise' & calcium & onions and garlic \\
\hline less alcohol: & less fat intake & \\
\hline relaxation therapy' & & \\
\hline
\end{tabular}

${ }^{1}$ conflicting evidence; 2 less than 3 glasses per day.

There is large evidence that obesity is linked with a higher prevalence of hypertension (128-129). MacMahon et al (130) estimared that approximately $30 \%$ of arterial hypertension can be attributed to obesity. Numerous studies of medical weight reduction have confirmed that weight loss is associated with a fall in blood pressure in severely obese hypertensive patients (131-133).

Before the introduction of thiazides in the late 1950s, it was known that salt restriction lowers blood pressure (134). Data from 11 adequately controlled studies showed that the average effect of $100 \mathrm{mmol} / \mathrm{day}$ reduction in sodium intake which was about half the usual intake - induced a $5.4 / 6.5 \mathrm{mmHg}$ fall in systolic and diastolic blood pressure, respectively (135). The higher the initial blood pressure (136) and the more rigid the salt restriction (137), the larger the fall in blood pressure. In contrast to the poor patient adherence to very rigid salt restriction, the acceptability and efficacy of moderate salt restriction $(60-100 \mathrm{mmol} / \mathrm{day})$ is good (138-139). However, such a modest salt restriction will induce only a significant fall in blood pressure in those patients, who are more sodium sensitive (127).

The effect of physical training on casual blood pressure has been extensively investigated. It has been recognized that blood pressure acutely decreases after physical exercise (140). It is not so clear whether exercise training may induce a more prolonged decrease in blood pressure. In a consensus statement by the World Hypertension League a small average decline of $4 \mathrm{mmHg}$ for systolic as well as diastolic blood pressure is assumed (141). However, the majority of controlled longitudinal studies did not show a decrease in blood pressure at rest (142-143). Recently 3 studies have been published on the influence of exercise training on ambulatory blood pressure. Two authors did not find any influence neither on casual nor on ambulatory blood pressure (144-145), whereas the 3rd author 
described a small decrease in diastolic blood pressure alone (146). Even if the effect of exercise training on blood pressure is small, physical exercise might play a role in the prevention of ill effects of hypertension. Indeed, exercise training decreases vascular resistance (144), which might be due to an increment in the size of the capillary bed of the skeletal muscle in combination with changes in regulatory effects of arterioles, i.e. decrease in sympathetic tone (147-149). This decrease in vascular resistance often did not result in a decrease in blood pressure because of a slight rise in cardiac index at rest (144,150-151). Regular exercise may also reduce the incidence of hypertension. Blair et al calculated that the relative risk for development of hypertension was 1.52 greater in men and women with low levels of physical fitness than in those with higher levels (152).

Most large-scale surveys find no higher blood pressures among those who consume 1-2 drinks per day. The consumption of more alcohol/day is associated with a higher prevalence of hypertension (153-155). One or two glasses of alcohol per day have been clearly associated with a lower mortality and morbidity rate from coronary heart disease than abstinence or higher amounts of alcohol (127). Therefore, for those who consume fewer than 3 drinks per day, no change in alcohol consumption seems necessary.

A variety of relaxation therapies such as yoga, psychotherapy, biofeedback, and transcendental meditation have shown to reduce blood pressure of hypertensive patients at least tansiently (127). A longterm antihypertensive effect was found in a study by Patel et al (156), but not in another study (157).

The INTERSALT study suggested a beneficial effect of the combination of different nondrug therapies. It was calculated that a reduction of daily sodium intake of $100 \mathrm{mmol}$, from current UK levels of 170 to 70 , an increase in potassium of $30 \mathrm{mmol}$, a reduction of body mass index of 5 , and a reduction of alcohol in heavy drinkers to less than 3 drinks a day would correspond to $11 \mathrm{mmHg}$ lower systolic blood pressure (158). Even if it were possible only to achieve half of these changes, mean blood pressure of the whole population would be lowered by $5 \mathrm{mmHg}$. which would correspond to an approximately $10 \%$ lower mortality from CHD and stroke (112). However, evidence for risk reduction from hypertension might never be established for some nondrug therapies, since efficacy of nondrug therapy is in general less effective than that of antihypertensive drugs and since they are also more difficult to monitor (127).

\section{2. pharmacological management}

\subsection{1. history}

Before the 1940s there was no effective way to lower raised blood pressure. Late in the 1940 s ganglion blocking agents were introduced (159). These drugs were useful in the treatment of malignant hypertension, but in non-malignant hypertension, the decrease in supine blood pressure was small. These drugs blocked sympathetic as well as parasympathetic nerves, thereby inducing numerous side effects. In 1957 thiazide diuretics were introduced and the use of reserpine became also popular. These 2 drugs could be given together, resulting in a fall in diastolic blood pressure of $10-20 \mathrm{mmHg}$ and, compared to older antihypertensive agents, they had relatively acceptable side effects. In 1959 the first adrenergic neuron blocker guanethidine was introduced. This drug was free of parasympathetic blockade. It acted through inhibiting the release of noradrenaline from nerve 
endings, and was - like the earlier ganglion blocking agents - not very effective in lowering supine blood pressure. In the early 1960s methyldopa was introduced, like reserpine a centrally acting drug, but more effective than either reserpine and thiazides.

The use of B-blockers as antihypertensive drugs was firstly reported by Prichard in 1964. Since that time their popularity has grown. In the 1980 s B-blockers were the most popular antihypertensive drugs in several European countries (160). In the last decade also calcium-antagonists and ACE-inhibitors became firstline antihypertensive agents (125).

\subsection{2. most important antihypertensive drugs currently used}

Most important antihypertensive drugs are shown in Table 1.9.

Table 1.9. Most important antibypertensive drugs currently used

\begin{tabular}{|c|c|}
\hline class & subclass \\
\hline B-blockers & $\begin{array}{l}\text { nonselective } \\
\mathbb{B}_{1} \text {-selective } \\
\text { with intrinsic sympathomimetic activity }\end{array}$ \\
\hline calcium-antagonists & $\begin{array}{l}\text { phenylalkylamines } \\
\text { benzothiazepines } \\
\text { dihydropyridines }\end{array}$ \\
\hline cliuretics & $\begin{array}{l}\text { thazides } \\
\text { thiazides + potassium sparing diuretics } \\
\text { aldosterone antagonists } \\
\text { loop diuretics }\end{array}$ \\
\hline \multicolumn{2}{|l|}{ ACE-inhibitors } \\
\hline$\alpha$-blockers & $\alpha_{1}$-blockers \\
\hline \multicolumn{2}{|l|}{ central $\alpha$-agonists } \\
\hline direct vasodilators & $\begin{array}{l}\text { hydralazine group } \\
\text { minoxidil } \\
\text { nitrates }\end{array}$ \\
\hline multiple action drugs & $\begin{array}{l}\text { S-blockade + vasodilation } \\
5 \mathrm{HT}_{2} \text {-antagonism }+\alpha \text {-blockade } \\
\text { central } 5 \mathrm{HT}_{1} \text {-agonism }+\alpha \text {-blockade } \\
\text { central } \alpha_{2} \text {-agonism }+ \text { imidazoline preferring receptor }\end{array}$ \\
\hline
\end{tabular}

Thiazide diuretios are the oldest antihypertensive drugs currently used. Initially, the increase in urinary salt and water excretion result in a reduced plasma volume and smaller preload for the heart. This reduction in preload results in a smaller cardiac output. However, after 8 weeks cardiac output is back to normal and intravascular volume is minimally reduced by $5 \%$ or Jess, but systemic vascular resistance is decreased (161). To prevent hypokalaemia, thiazide diuretics are often combined with potassium sparing diuretics. The loop diuretics such as furosemide are less effective in controlling blood pressure compared to an equivalent cose of hydrochlorothiazide (162).

Although the antihypertensive mechanism of B-blockers is still not fully understood, the effect is mainly due to $B_{1}$-blockade. B-blockers with vasodilating properties might be of interest since they do not increase vascular resistance (163). 
Three groups of calcium-antagonists are currently used: the phenylalkylamines (verapamil), the benzothiazepines (diltiazem) and the dihydropyridine calciumantagonists. The 3 groups differ in vascular selectivity. Verapamil is as (non)selective for the heart as for the vessels; diltiazem is 7 times more selective for the vessels, but still has a marked effect on the heart. The dihydropyridines are more selective for the vessels, but within this group vessel-heart selectivity ratio differs largely: e.g. 14 with nifedipine and 118 with felodipine (164).

The short-term antihypertensive effect of ACE-inhibitors may result from vasodilation by inhibition of the vasoconstrictor angiotensin II, by higher levels of the vasodilator bradykinin and by the loss of facilitated sympathetic nerve action (165). A lot of attention is paid to the long-term effects of ACE-inhibitors as inhibitors of the growth factor angiotensin II. This particular property might be important in cardiac and vascular remodeling and atherogenesis during hypertension (160).

Alpha-blockers have the theoretical advantage of improving the lipid profile in the plasma. All lipid subfractions and also the apolipoproteins are favorably influenced $(161,167)$. The main disadvantage of alpha-blockers is the first dose hypotension and syncope, but also during chronic therapy orthostatic hypotension may occur. Orthostatic hypotension is reported in up to $20 \%$ of patients, while syncope occurs in less than $2 \%(168)$. Orthostatic side effects are less frequent in second generation $\alpha$-blockers like doxazosin and terazosin than during prazosin (168).

Other antihypertensive drugs are less frequently used. Most of the centrally acting antihypertensive drugs have sedative side effects. Direct vasodilators like hydralazine and minoxidil cannot be used in monotherapy due to reflex tachycardia and salt and water retention (161). Nitrates predominanty dilate the smaller arteries (169), resulting in a smaller pulse pressure. Nitrates might be of interest especially in patients with isolated systolic hypertension since they reduce systolic pressure, without substantial decrease in diastolic pressure (170). The preservation of the diastolic pressure might be important for a good coronary circulation in these patients (171).

In the last few years also some new drugs with combined action were introduced like urapidil and ketanserin. Due to serotonin 2 antagonism ketanserin could inhibit platelet aggregation (172) and might be protective against coronary events (173). The 1988 Joint National Committee on Detection, Evaluation and Treatment of High blood Pressure proposed 4 classes of anthypertensive drugs as firstline drugs: diuretics, fs-blockers, calcium-antagonists and ACE-inhibitors. Duc to its beneficial effect on lipids, authors such as Kaplan judge selective $\alpha_{1}$-blockers as suitable for initial therapy as the 4 aforementioned classes (168).

\subsection{3. results of pharmacological treatment of hypertension}

In contrast to the old antihypertensive drugs, modern antihypertensive drugs lower blood pressure effectively. Most antihypertensive agents of the different therapeutic classes exhibit similar response rates, approximately 30 to $60 \%$ (174-175). Using the approach of sequential monotherapy and testing 2 or even 3 types of drugs consecutively, a higher number of patients, possibly reaching $70-80 \%$ of the hypertensive population, could be treated by monotherapy alone (175).

The benefits of effective treatment in malignant hypertension rapidly became clear $(30,176)$. In non-malignant hypertension results were not so pronounced. From 
a meta-analysis of 14 placebo-controlled, randomized trials in subjects with nonmalignant hypertension pharmacological treatment induced a $42 \%$ reduction in the incidence of stroke and a $14 \%$ reduction in coronary events (177). The reduction in stroke is better linked to the reduction in blood pressure than atherosclerosis (30). Recent data from patients with isolated systolic hypertension suggest a reduction by $36 \%$ in stroke and of $27 \%$ in coronary events $(41)$.

A further reduction especially in coronary events would be desirable not only because of the small risk reduction obtained but also because the incidence of coronary heart disease (Table 1.10) is $2-5$ times higher than that of stroke $(30,31)$.

Table 1.10. Mortality in treated bypertensive patients (30)

\begin{tabular}{|l|l|}
\hline ill effect & mortaliry (all causes) \\
\hline ischaemic heart discase & $36 \%$ \\
\hline stroke & $18 \%$ \\
\hline other cardiovascular discase & $8 \%$ \\
\hline renal failure & $3 \%$ \\
\hline
\end{tabular}

Different reasons for the rather poor effect of therapy on morbidity and mortality have been suggested such as poor blood pressure control and the adverse effects of the anthypertensive treatment itself promoting ill effects.

\subsection{4. drug adherence}

Faulty therapeutic compliance is probably the main cause of poor blood pressure control in the community (178). In the USA in the 1970s, the rule of the halves applied: half the hypertensives were unknown; of those that were known, only half were on treatment; of those on treatment only half had blood pressure under control. In other words, about $12 \%$ of hypertensives were treated satisfactorily (116). By 1985 , more than $75 \%$ of those with high blood pressure were aware of it and $79 \%$ were taking some action to control it (116). Other authors estimated that of those patients who continue to attend clinics for hypertension, one half to two thirds take enough pills to lower their blood pressure to normal (179-180). Compliance with an antihypertensive drug regimen is related to frequency of dosing and was markedly better on a once daily regimen than on a three times daily regimen (181-182). Compliance appears to be better in rural family practices than in urban clinics. In an urban clinic only $38 \%$ of patients took more than $75 \%$ of their pills (183), whereas this was $79 \%$ in a rural setting (184). Although there is some evidence that the intensity with which the physician prescribes medication correlates with the level of blood pressure control, it is also clear that as more pills are prescribed, a lower portion is taken (185). Compliance has been improved by different approaches such as a reduction of the number of pills, simplification of the drug regimen, maximum use of nondrug therapies, increased family involvement, home monitoring, self-help groups and patient rewards (180). Since patients with mild-to-moderate hypertension are often asymptomatic (186), it is very important that, in order to assure good patient compliance, antihypertensive drugs should not impair the quality of life. Better tolerated antihypertensive drugs with fewer side effects should therefore be helpful (187). 


\section{2. prevention of ill effects of hypertension}

\subsection{Treatment and prevention of cardiac hypertrophy.}

In general, ventricular arrhythmias have been shown to diminish in parallel with the reversal of left ventricular mass (188-189). Preliminary data on 166 hypertensives followed up for 5 years showed that cardiovascular events occurred in only $6 \%$ of patients whose left ventricular mass decreased or was unchanged from baseline in contrast to $16 \%(p<0.05)$ of parients whose left ventricular mass increased from baseline (190). It was concluded from this study that reduction of left ventricular mass during treatment of essential hypertension is associated with improved prognosis, and that measurement of left ventricular mass index may provide a better indication of the efficacy of treament than the level of blood pressure.

In a meta-analysis of 109 treatment studies Dahlof et al showed that all 4 classes of firstline anthypertensive drugs can reverse left ventricular hypertrophy in hypertensive patients (191). The average reduction in left ventricular mass was more pronounced with ACE-inhibitors compared to diuretics, calcium-antagonists and I - blockers (Table 1.11). In this study no difference has been made between the B-blocker subclasses. This study also includes B-blockers with ISA, which are thought not to cause regression of left ventricular hypertrophy (62). As a consequence the average effect of the other subclasses of is-blocking agents might be better. Also other antibypertensive drugs like $\alpha$-blockers and methyldopa can reverse left ventricular hypertrophy while vasodilators like minoxidil and hydralazine did not (64).

Table 1.11. Elfect of antitypertensive drugs on cardiac bypertrophy (191)

\begin{tabular}{|l|c|}
\hline drug & change in left ventricular mass \\
\hline diuretics & $-21.4(-490-6)$ \\
\hline Calcimm-antagonists & $-26.9(-420-12)$ \\
\hline B-blockers & $-22.8(-3860-8)$ \\
\hline ACE-inhibitos & $-44.7(-660-23)$ \\
\hline
\end{tabular}

- mean and $95 \%$ contidence intervals expressed in gram.

Recent survival trials on left ventricular dysfunction, such as the Veteran Administration-Heart Failure Trial (V-HeFT) I and II (192-193) have clearly shown that vasodilator therapy can prolong life expectancy in patients with severe left ventricular dysfunction. However, the V-HeFT II study showed that the ACEinhibitor enalapril did increase survival much more than the combination of hydralazine-isosorbide dinitrate. In addition, enalapril provided a proportionately greater benefit in patients with dilated cardiomyopahy than in those with underlying ischaemic heart disease (192). This might indicate a predonninant action of ACEinhibitors on the remodeled heart. The benefical effect of ACE-inhibitors on mortality rates in patients with cardiac dysfunction has also been shown in the Cooperative North Scandinavian Enalapril Survival Study (CONSENSUS I; 67), the Study On Left Ventricular Dysfunction, using enalapril (SOLVD; 68), and in the Survival and Ventricular Enlargement Trial (SAVE; 194), using captopril. 
Large survival trials have investigated the influence of drugs on reversal of cardiac hypertrophy. However, little is known on the effect of antihypertensive drugs in preventing cardiac hypertrophy. Mostly cardiac hypertrophy develops more slowly in hypertension than after myocardial infarction, but the process might be very similar. In 2 studies - CONSENSUS $I 1$ (195) and SMILE (Survival of Myocardial Infarction Long-term Evaluation; 196) trials - which, initiated ACE-inhibitor therapy within 24 hours after myocardial infarction and registered the outcome for only 6 to 12 months, no beneficial effect on mortality was seen. Since cardiac remodeling takes some time (months) to appear, the lack of a positive outcome in these 2. studies might be due to the relatively short follow up time. This is supported by the observation that the differences in mortality rates in the SAVE trial only becane clear after 1 year of treatment. In addition, in rats ACE-inhibitors showed a deleterious effect if given early after myocardial infarction, with a strong suggestion that the healing process of the hear after infarction was altered by ACE-inhibition (197). This could be an alternative explanation for the lack of a positive outcome in the CONSENSUS II and SMIIE trials.

In conclusion, all firstline (and also some other) antihypertensive drugs con reverse cardiac hypertrophy , but ACE-inhibitors are more powerful in this respeci. Survival studies in patients with cardiac dysfunction have shown a beneficial effect of ACE-inhibiors. However, no data are available on the effect of olher drugs on survival in this condition. Prevention of cardiac hypertrophy is more difficult to study and no good' data are available. However, primary prevention mighi be more important, sinee it is not clear whether fibrosis of the heart can be reversed.

\section{2. prevention of ischaemic cardiovascular events}

Three strategies can be imagined to prevent ischaemic cardiovascular events: (1) prevention of atherogenesis, (2) prevention of plaque rupture and (3) prevention of occlusive thrombosis and vasospasm.

2.2.1. Prevention of atherogenesis is complex for many reasons such as: [1] the origin of vascular injury is multifactorial; [2] many different cells and growth factors are involved, and [3] since carly lesions already initiate at puberty, prevention should start preferably early in life. Nowadays prevention of atherogenesis regards mainly the prevention of risk factors like smoking, hypertension, hypercholesterolaemia, insulin resistance, emotional stress, and obesity. Influences on risk factors are discussed in section 2.2.4.

Antiproliferative properties of different substances like heparin (198-200), fish oil (201) and suramin (202) are being studied. Also of potential therapeutic value are other proliferative inhibitors like angiotensin II inhibitors (converting enzyme inhibitors and angiotensin II receptor antagonists). Altough some ACE-inhibitors might prevent intimal thickening in atherogenic animal models, their value in prevention of atherogenesis in man is not established, yet (203).

Another target can be to influence the role of lipids in atherogenesis. In this respect, the importance of lipoprotein modifications, especially oxidation, has renewed interest in antioxidants (204-205). In this context, probucol has been demonstrated to slow the progression of atherosclerosis in hyperlipidaemic rabbits (206). 
Almost all classes of antihypertensive drugs tested have shown some favorable antiatherogenic effect on some experimental model of hypercholesterolaemia (203). Of all antihypertensive drugs calcium-antagonists are best studied for their possible anti-atherogenic potency. They retard the progression of atherosclerosis (207). The mechanisms of action by which calcium-antagonists exert their anti-atherosclerotic effect have not been completely clucidated (208). In experimental models calciumantagonists can beneficially modify many factors in the atherosclerotic process, such as endothelial damage and necrosis, platelet aggregation, release of plateletderived growth factor, migration from media to intima, proliferation of smooth muscle cells, protein collagen synthesis, binding of lipoproteins to glycosaminoglycans (209). Recent reports indicate that nifedipine, verapamil and diltiazem exert antiperoxidative effects on membrane lipids, preventing oxidation of LDL, a modification thought to confer atherogenic properties to the lipoprotein (208). It is possible that the major anti-atherogenic effect of calcium-antagonists is related to their antiperoxidative properties.

\subsubsection{Prevention of playue rupture}

Prevention of plaque rupture could be realized by stabilization of a weak plaque or by decreasing stresses at the plaque and the vascular wall. Since weak lipid-rich plagues are more rupture-prone, stabilization could be achieved by decreasing cholesterol levels. This hypothesis is supported by the results of studies on the effect of lipid lowering. In contrast to the small regression in atherosclerotic plaques on angiography, the reduction in the number of cardiac events was quite considerable in (wo of these studies (210-211). Therefore, it was suggested that the small increase in luminal diameter measured by means of angiography is more likely a marker of more important regression of the smaller, lipid-rich plaques. These smaller lipid-rich plaques may be undetectable angiographically, but represent the main clinical contributors to the acute coronary events (212).

The role of physical stress on plaque disruption needs further investigation. The major stresses on the vessel wall (circumferential and shear stress) are discussed in part II. As already mentioned, coronary arteries additionally suffer from stress of the pumping heart. Agents that lower heart rate and/or contractility might decrease coronary stress. B-Adrenoceptor antagonists can provide secondary (213-214) and probably also primaty (215) prevention of myocardial infarction. This favorable effect has been ascribed to a decrease of the stress on plaques and the risk of plaque rupture in the coronary arteries (216). Also non-dihydropyridine calciumantagonists like verapamil (217) and dilriazem (218) might have their secondary prevention effect on the basis of the inhibition of plaque disruption.

2.2.3. The third strategy is the prevention of thrombosis and vasospasm. Since. thrombus formation seems to be an important factor in the progression of coronary disease and in the conversion of chronic to acute events after plaque disruption, a promising approach is the prevention of these processes with the use of antithrombotic therapy. Antiplatelet agents might offer some promise in preventing the progression of small coronary atherosclerotic plaques (219), but the most beneficial effect of antiplatelet and anticoagulant agents has, been observed in the prevention of acute coronary events (220-223). The best-studied, least toxic, and most widely used antithrombotic agent is aspirin. Aspirin interferes with platelet 
activation. On the other hand anticoagulant agents interfere only partially with the coagulation system and are ineffective against platelet activation. In coronary artery disease, the overall antithrombotic effectiveness of aspirin and anticoagulant agents is clinically similar (224), Combination therapy with low-dose aspirin and anticoagulant agents may have additive effects (212).

Inhibition of coronary vasospasm can be achieved by different vasodilating drugs. Nitrates and calcium-antagonists are the most currently used. They have a direct vasodilating effect on smooth muscle cells of the vessel wall. In addition, calciumantagonists can abolish endothelin-induced vasoconstriction (225). Also ACEinhibitors can attenuate vasoconstriction in patients with coronary ancty disease by increasing levels of vasodilating substances like prostacyclin and bradykinin, by decreasing levels of the vasoconstricting angiotensin 11 (226), and by attenuating sympathetic coronary vasoconstriction (227).

\subsection{4. influence on risk factors for atherosclerosis.}

Recently the influence of different antihypertensive drugs on insulin resistance have been shown. B-Blockers like metoprolol and atenolol and the diuretic hydrochlorothiazide increase insulin resistance. Calcium channel antagonists verapamil and diltiazem are metabolically neutral, whereas nifedipine has a negative effect. Insulin resistance improves with the $\alpha$-blocker prazosin and with the ACE-inhibitor captopril (228). In addition, dilevalol, a B-blocker with vasodilating properties, also has a beneficial effect on insulin resistance (229). It has been suggested (230) that the beneficial effect of captopril on insulin resistance might be due to the increased bradykinin, which promotes the insulin-induced glucose uptake into skeletal muscle.

Two studies have shown that the B-blockers atenolol and propranolol can convert type 'A' behaviour in a mellower type ' $B$ ' behaviour (231-232). The potential value of such a conversion can be deduced from the results of the Recurrent Coronary Prevention Project. This study showed that after a 4.5 years follow up period in postmyocardial patients, cardiac events and deaths were reduced by $39 \%$ in those patients, whose type 'A' behaviour was modified towards a type 'B' pattern (233). There is a continuous and graded relation between serum cholesterol levels and coronary heart disease death, with a 4 to 6-fold increase in risk from levels of $160 \mathrm{mg} / \mathrm{dl}$ to the highest cholesterol values (234-235). Lowering cholesterol in patients with hypercholesterolaemia decreases the risk of atherosclerotic complications and reduces mortality from coronary artery disease (210-21 1, 236-239). However, in a meta-analysis of 18 observational studies Jacobs et al showed the existence of a U-shaped relation between serum cholesterol and total mortality in men, and a flat relation in women (Table 1.12). This $\mathrm{U}$ shape resulted largely from a positive relation of total cholesterol with coronary heart disease death and an inverse relation with deaths caused by some cancers (e.g., lung but not colon), respiratory disease, digestive disease, trauma and residual deaths. One of the explanations might be that total cholesterol is lowered by some disease conditions themselves, such as wasting in cancer and pulmonary disease (240). But also the pattern of stroke is changed in patients with low serum cholesterol. Serum cholesterol is strongly related to death from non-haemorrhagic stroke. But men in the lowest category of serum cholesterol $(<4.14 \mathrm{mmol} / 1 ; 160 \mathrm{mg} / \mathrm{dl}$ ) had higher death rates from both subarachnoid and intracerebral hacmorrhage than other 
men (116). The association between low cholesterol and intracerebral haemorrhage has also been observed in Japan (241-2.42), where the data pointed to a Ushaped relation.

Table 1.12. Risk ratios for deaths occurring 5 years after study baseline. (240)

\begin{tabular}{|l|l|l|l|l|}
\hline $\begin{array}{l}\text { blood } \\
\text { cholesterol } \\
(\mathbf{m g} / \mathrm{d})\end{array}$ & all causes & $\begin{array}{l}\text { total } \\
\text { cardiovascular }\end{array}$ & $\begin{array}{l}\text { total } \\
\text { noncardiovascular }\end{array}$ & $\begin{array}{l}\text { total } \\
\text { noncardiovascular } \\
\text { noncancer }\end{array}$ \\
\hline$\leqq 160$ & $1.17(3.6)$ & $1.04(0.8)$ & $1.18(2.3)$ & $1.32(5.3)$ \\
\hline $16 !-199$ & 1.00 & 1.00 & 1.00 & 1.00 \\
\hline $200-239$ & $1.02(0.8)$ & $1.16(3.6)$ & $0.95(-1.8)$ & $0.89(-2.5)$ \\
\hline$\geq 240$ & $1.14(5.1)$ & $1.48(9.5)$ & $0.95(-1.2)$ & $0.87(-3.6)$ \\
\hline
\end{tabular}

pooled data of 18 studies; data of men; ( ) number of standard deviations that the estimated hazard ratio departs from 0 .

In a review of six randomised controlled primary prevention trials, Muldoon et al (243) found that lowering of raised serum cholesterol in middle-aged subjects by diel, drugs, or both was also associated with a significant decrease in the number of eleath from coronary heart discase but not in total deaths. When the World Health Organisation Study (244) results were excluded, there was no association between cholesterol reduction and cancer (243). In the World Health Organisation Study clofibrate was used. The use of this drug might be related to cancer (244). However, there was a significant increase (nearly $100 \%$ ) in mortality due to suicides or violence: compared with control subjects, treated groups had 28 fewer deaths per 100000 from coronary heart disease and 29 excess deaths from suicides, homicides and accidents. The increase in deaths due to violence was present in all 6 trials suggesting that these findings are not due to chance alone. As with the large observational trials and the primary prevention trials, noncardiovascular deaths increased significantly in a meta-analysis of 7 secondary prevention trials (239). In conclusion: Randomised controlled trials have demonstrated the benefit of cholesterol reduction as primary prevention to reduce coronary heart disease in midile-aged men. There is no clinical trial evidence that primary prevention will redice overall motality (245). The protection provided against coronary heart disease through cholesterol reduction may be the greatest in patierts with the highest serum cholesterol concentrations before treament (as was noted in the Worki Health Organisation study). If so, promotion of long-term survival might be best achieved by targeting intervention efforts primarily at those people who are at particularly high risk for dcath from caronary heart disease: those with exceptionally high serum cholesterol concentrations, history of coronary disease, or several risk factors in addition to hypercholesterolaemia (243). This view is also supported by a cost-effectiveness analysis (245). Further fong-term studies and siudies in women and clderly patients are needed. 
Some antihypertensive drugs negatively influence the lipid profile, but they may exert some favorable effect on some experimental models of hypercholesterolaemia (203). For example fi-blockers may decrease the serum level of the protective HDL cholesterol and increase triglycerides, but they show an anti-atherogenic effect in animal models (246). What could be the reason for this surprising observation? Clinical and experimental data suggest that B-blockers might increase the size of the LDL particles, Large LDL particles are less atherogenic (247). In addition. B-blockers decrease LDL-binding to arterial proteoglycans, resulting in less accumulation of LDL in the arterial wall (247), an anti-atherogenic effect. Due to the increase in LDL size, the triglyceride core of the LDL particle increases. As the larger LDL is less atherogenic, one might speculate that B-blocker-induced increase in triglycerides might actually signal a beneficial effect rather than a negative one (215). This example also suggests that drug-induced presence of a risk factor might not have the same meaning as the spontaneous presence of that risk factor.

\section{3. prevention of stroke.}

About $40 \%$ of strokes can be attributed to systolic blood pressure of more than $140 \mathrm{mmHg}$ (44), A high risk approach that sought to detect and treat those with casual systolic blood pressure of greater than or equal to $170 \mathrm{mmHg}$ would potentially save $35 \%$ of the excess deaths. However, most of the deaths atuributable to a raised blood pressure come from the majority of the population with modest. increases in pressure. Therefore, only an approach that lowered average population blood pressures would reduce the incidence of stroke associated deaths attributable to raised blood pressure (116). In the large treatment trials, people over 65 derive benefit from treatment (248); there are no grounds for assuming that the rise of blood pressure with age is "normal" and requires less attention in the elderly (116).

The majority of strokes are ischaemic strokes (249). Therefore, risk factors for stroke are about the same as the risk for atherosclerosis and serious cardiac events (249-250). Approaches to prevention of atherosclerotic disease have been discussed previously and might also be beneficial in preventing ischaemic stroke. Secondary prevention of ischaemic stroke can be made by antiplatelet drugs. No antiplatelet drug is clearly better, cheaper and less toxic than aspirin (249). Thromboxane $A_{2}$ antagonists and/or synthetase inhibitors can be prescribed for patients who cannot tolerate aspirin. The recommended close of aspirin is $300 \mathrm{mg}$ for almost instant inhibition of platelet aggregation, followed by $75.150 \mathrm{mg}$ a day, presumably indefinitely (249). Studies on the value of aspirin in the cerebral condition are being established (251).

The priority of stroke prevention has to be on the one hand control of high blood pressure in hypertensive patients and on the other hand reduction of blood pressure in the whole population. Also the reduction of risk for atherosclerosis like reduction of smoking, reduction of excess alcohol to less than 3 glasses per day and dietary measures may likewise have an impact on stroke (116).

\section{4. prevention of renal failure.}

The reported mortality from hypertensive renal disease has declined dramatically over the past 20 years in both sexes (252). Diuretics have no direct effects on renal haemodynamics. Renal blood flow and glomerular filtration rate are not affected 
by $\alpha$-blockers, but tend to be reduced by B-blockers. With selective $\beta_{1}$-blockers, this effect decreases with time. In general, these effects are not a problem, except for hypertensives with increased peripheral resistance and declining cardiac output. In these patients renal function may be altered. Calcium-antagonists and ACEinhibitors decrease renal vascular resistance. ACE-inhibitors and calcium-antagonists might diminish renal injury in experimental models (124). Long-term benefits of these agents in humans still need to be proven.

Lessons of the past 20 years have taught us that lowering blood pressure by any means (pharmacological and non-pharmacological) helps in reducing renal damage (124). 


\section{this thesis}

It can be concluded with the 1988 Joint National Committee on Detection, Evaluation and Treatment of High blood Pressure, that it is not enough to lower blood pressure in hypertensive patients; the goal of treatment should be to improve longevity, reduce minor complications, and improve the quality of life (125). Longevity can be improved by prevention of the ill effects of hypertension. The most important ill effects are stroke, (coronary) atherosclerotic disease, and cardiac hypertrophy leading to arrhythmia and/or heart failure.

The prevention of ill effects and the preservation of quality of life go beyond the reduction of the blood pressure in the restricted sense. Therefore, prevention of ill effects and preservation of quality of life are called non-antihypertensive aspects of antihypertensive treatment. Nevertheless, these aspects can be substantially influenced by the reduction in blood pressure.

The ill effects and their management have been introduced in the previous chapters. Most research in this regard has focussed on prevention of atherosclerotic disease. Studies have been made on the effects of reduction of hyperlipidaemia and of antithrombotic treatment. However, there are also arguments for an important role of physical factors in the ill effects of hypertension. For example, the incidence of stroke decreases with decreasing blood pressure (114); systolic ventricular wall stress is thought to stimulate cardiac hypertrophy (48); in the response-to-injury hypothesis, physical factors might induce intimal lesions of the vessel wall, which might - together with other promoting factors - lead to the formation of an atherosclerotic plaque $(69,71-72)$. Even more important in atherosclerotic disease might be the physical stress on the arterial wall, which might induce plaque rupture leading to rapid progression of plaques or to acute thrombotic occlusion of the vessel (77-78). Part II of this thesis will deal with these physical factors and the influence of antihypertensive drugs in this respect. Chapter 1 reviews the physical factors on the vessel wall and the influence of antihypertensive therapy in this regard. Subsequently, non-invasive investigation of the effect of two antihypertensive drugs on the vessel wall properties of the common carotid antery is presented: chapter 2 investigates the effect of the non-selective calcium-antagonist verapamil and chapter 3 the effect of nebivolol, a selective $B_{1}$-adrenoceptor antagonist with vasodilating properties.

Preservation of quality of life is an important aim of medicine in general and an important tool to improve drug adherence. Perceived quality of life is the balance between positive and negative influences of a treatment. Positive influences on quality of life are mostly the disappearance of symptoms of the disease. The negative influences are in general side effects of the drug. Since mild-to-moderate hypertension is in general asymptonatic, side effects of drugs may easily reduce quality of tife. Therefore, the influence of antihypertensive therapy on quality of life is very important for the hypertensive patient and also for a successful antihypertensive treatment. This non-antihypertensive aspect of antihypertensive treatment is discussed in part III. Firstly, the Inventory of Subjective Health (ISH), a general health profle is introduced. In chapter I the effect of nebivolol on quality of life as measured with the ISH questionnaire is compared with the effect on a 5-point perceived health scale and with the influence on side effect diaries. 
Chapter 2 compares the quality of life during antihypertensive therapy with the highly selective $B_{1}$-adrenoceptor antagonist bisoprolol with the quality of life during treatment with the ACE-inhibitor enalapril. Chapter 3 compares the influence on exercise tolerance of two B-blocking drugs (atenolol and nebivolol) and reviews the effect of antihypertensive therapy on exercise tolerance. Finally part IV discusses different aspects of current antihypertensive treatment and formulates issues for future research. 


\section{PART II. \\ INFLUENCE OF PHYSICAL FACTORS ON THE VESSEL WALL}

In part I evidence was discussed that physical stress at large arteries may be important in the development of cardiac hypertrophy, progression of atherosclerosis, occurrence of acute coronary events and stroke. Morbidity $(42-45)$ and mortaliry (44) from hypertension are better correlated with systolic than with diastolic blood pressure, indicating a major role for the pulsatile stress in the ill effects of hypertension. The pulsatile stress is a circumferential stress which induces degeneration of the vascular media (253). Pulsatile stress in the vessel wall is a physical factor determined by a number of variables, which are discussed in chapter 1. Shear stress is the tangential stress at the vessel wall and acts predominanty on endothelial cells. Shear stress can be subdivided into a meari and a pulsatile shear stress. Mean shear stress is determined by variables like blood viscosity, mean blood velocity and the diameter of the vessel. Pulsatile shear stress is also influenced by vessel wall properties.

Chapter 1 reviews how antihypertensive drugs - in addition to their blood pressure lowering effect - influence physical factors, which seem to determine risks associated with hypertension. Chapter 2 and 3 describe experiments on the noninvasive measurement of vessel wall properties of the common carotid artery and the effect of 2 antihypertensive drugs (verapamil and nebivolol, respectively) on these large artery properties.

\section{chapter 1. physical factors on large arteries and antihypertensive therapy ${ }^{1}$}

\section{1. pulsatile stress in the arterial wall}

Pulsatile stress can be compared with forces inducing material fatigue (253). Important stress factors are peak force [systolic blood pressure], change in force [pulse pressure], velocity of this change, and frequency [heart rate] (253). Suress factors and their determinants are shown in Table 2.1.

Table 2.1. Determinants of pulsatile circumferential stress

\begin{tabular}{|l|l|}
\hline stress factor & determinants \\
\hline $\begin{array}{l}\text { peak stress } \\
(=\text { systolic pressure) }\end{array}$ & $\begin{array}{l}\text { mean arterial pressure } \\
\text { pulse pressure }\end{array}$ \\
\hline $\begin{array}{l}\text { change in stress } \\
\text { : pulse pressure) }\end{array}$ & $\begin{array}{l}\text { vessel wall propertics } \\
\text { stroke volume } \\
\text { (pulse wave reflections) }\end{array}$ \\
\hline $\begin{array}{l}\text { velocity of change } \\
(=\mathrm{dp} / \mathrm{dt})\end{array}$ & $\begin{array}{l}\text { vesse! wall properties } \\
\text { cardiac inotropy }\end{array}$ \\
\hline stress frequency ( = heart ratc) & cardiac chronotropy \\
\hline
\end{tabular}

${ }^{1}$ based on: LM Van Bortel, AP Hoeks, MJ Kool, HA Struykei Boudier. Introduction to large artery properties as a target for risk reduction by antihypertensive therapy. I Hypertens 1992, 10(suppl 6): $123-126$. 
Vessel wall properties of large arteries are important determinants of this pulsatile stress, since a decrease in compliance will affect 3 stress factors : peak force, change in force, and velocity of this change.

\subsection{Vessel wall properties of large arteries}

Vessel wall distensibility and arterial compliance are importan vessel wall properties. Distensibility is a two-dimensional entity defined by the relative change in cross-sectional area of the vessel per change in pressure. Distensibility is an important determinant of the pulsatile stress on the vessel wall. Compliance is a threedimensional entity, defined by the change in volume per change in pressure. Compliance reflects the buffering capacity of the large vessels and is therefore an important determinant of the afterload for the heart. In the literature, the term compliance is often used for compliance as well as distensibility. This confusion remained until a few years ago, since no means were available to measure distensibility as well as compliance accurately. A few years ago, in Maastricht as well as in Lausanne, non-invasive echo-Doppler techniques were developed, which can measure vessel wall properties of large arteries accurately. With the technique developed by Dr Hoeks and co-workers in Mastricht, large artery diameter and change in diameter during the heart cycle of different large arteries can now be measured. From these data and the pulse pressure, both distensibility and compliance can be calculated. Chapters 2 and 3 describe two studies, as examples of the results obtained with this (first generation) non-invasive technique.

\section{Table 2.2. Effect of firstline antibyperlensive drugs on stress determinams}

\begin{tabular}{|c|c|c|c|c|c|}
\hline determinant & MAP & $M:$ & PWR & $\mathrm{CI}$ & IIR \\
\hline $\begin{array}{l}\text { calciumantagonists } \\
\text { - dihydropyridine } \\
\text { - non-dihyctropyridine }\end{array}$ & : & : & (") & $\cdot$ & • \\
\hline $\begin{array}{l}\text { B-blockers } \\
\text { - non-selective } \\
\text { - selective } \beta_{1} \\
\text { - vasodilating" }\end{array}$ & ; & $\dot{.}$ & - & $\dot{*}:$ & $:$ \\
\hline ACE-inhibitors & - & - & • & & \\
\hline diuretics & * & & & $(*)$ & \\
\hline
\end{tabular}

" B-blockers with vasodilating properties without ISA MAP is mean arterial pressure; vessel walt properties are arterial compliance ( $A$ C) including the effect on pulse wave reflections (PWR); $C I$ is cardiac inompy: HR is heart rate. " is a benefictal effect decreasing pulsatile stress: $(*)$ a beneficial effect might be present.

In hypertensive (254) and aging subjects (255-256) arteries become less elastic leading to a decrease in distensibility and compliance. Large artery compliance can be increased by antihypertensive drugs such as calcium-antagonisis (257-261), ACE-inhibitors (261-263), B-blocking agents with vasodilating properties (264-268) and nitrates $(261,269)$. Apart from metoprolol $(270)$, all $B_{1}$-blockers $(266,271)$ also improve compliance. At higher doses $\AA_{1}$-blockers loose selectivity. This might be an explanation for the difference between metoprolol (100 $\mathrm{mg}$ twice daily) and 
other selective $B_{1}$-blockers on large artery compliance. Since a higher blood pressure, by itself, decreases arterial distensibility (169), the beneficial effect of some antihypertensive drugs on large artery compliance might, in part, be due to the decrease in blood pressure. Despite a marked fall in blood pressure, the nonselective B-blocker propranolol (264), diuretics (272), vasodilators of the hydralazine group (273) and the centrally acting alpha agonist clonidine (274) do not increase arterial compliance. The effect of alpha-blocking agents is not clear. On the one hand ketanserin $\left(\alpha+\right.$ serotonin $_{2}$-antagonist) improves arterial compliance $(270)$, while urapidil ( $\alpha$-adrenoceptor antagonist + central serotonin $1 \mathrm{~A}^{-}$ agonist) does not (268).

It is clear that the effect of different antihypertensive drugs on large artery compliance is not uniform. Is this effect also dependent on the condition of the vessel wall? In a recent study, described in chapter 2 , this question was addressed using the calcium-antagonist verapamil (258). The results of this study suggest that verapamil given for 1 month is less effective on arterial compliance when siructural changes are present. All studies concerning the effect of antihypertensive drugs on large artery compliance mentioned are acute or short-term chronic studies (up to 3 months of therapy). Consequenty, effects on arterial compliance in these studies are likely to be due to functional rather than to structural changes. It is not clear whether a decrease in compliance due to structural changes can be improved. Prospective long-term studies are not available.

\section{2. pulse wave reflections}

In conditions with substantially decreased arterial distensibility, as in hypertensives and older subjects, pulse wave velocity increases and early wave reflections, originating from artery transitions with different impedance, can boost the systolic pressure in the ascending aorta (169), resulting in a substantially higher systolic stress on the heart and central arteries (169). Recent studies have shown that this distorted wave reflection can be reduced or almost abolished by some antihypertensive drugs. Beneficial effects in this respect have been described for nitrates (275-276), calcium-antagonists and ACE-inhibitors (169). Also B-blocking agents with vasodilating properties like dilevalol might decrease wave reflections (260). Nitrates have a particular property. Low-dose nitrates selectively dilate smaller arteries with almost no effect on arteriolar tone (169). This results in a decrease in systolic stress and pulse pressure in central large arteries without substantial decrease in mean and diastolic pressure $(170,269)$. These drugs might be useful in the treatment of isolated systolic hypertension, since - apart from a reduction in the harmful systolic pressure - during nitrate treatment diastolic pressure is preserved or may rise, resulting in a better perfusion of the heart.

\section{3. other determinants of pulsatile stress}

Determinants of pulsatile stress factors are shown in Table 2.1. Mean arterial pressure can be influenced by decreasing arteriolar tone (systemic peripheral resistance) or by decreasing cardiac output. This decrease in mean arterial pressure is achieved by classical antihypertensive therapy. A lower stroke volume decreases pulse pressure. Velocity of change in pulse pressure can be decreased by a slower cardiac contraction. Stress frequency is coupled to heart rate. Consequently (Table 2.2), beta-blocking drugs, verapamil and diltiazem might have a beneficial effect on 
large arteries by decreasing heart rate (stress frequency) and cardiac inotropy (stroke volume and velocity of change in pulse pressure).

\section{2. shear stress}

Plaque formation correlates best with low mean shear stress and with oscillations in shear stress direction, and not with relatively high levels of wall shear stress where flow remains laminar and unidirectional (72). At arterial bifurcations, shear stress is relatively high in the region of the flow divider, while preferential sites of plaque formation are the lateral walls opposite to the central how divider, regions of low shear stress (72).

With low shear stress particle residence time is increased. This means that the duration of exposure of the endothelial surface to circulating atherogenic agents or to conditions favoring transendothelial diffusion of atherogenic particles is increased in low shear regions (277). In this respect accumulation of monocytes, thrombocytes, and granulocytes on endothelial lumen surfaces may also be increased by a low wall shear stress (278-279). Endothelial cells exposed to high shear stress are mechanically stiffer than control cells. The increase in stiffness might strengthen endothelial integrity, which may protect against atherosclerosis (280). In addition, microfilament bundles may modulate endothelial permeability and prevent influx of nacromolecules such as low density lipoproteins or cellular components like monocytes. In regions of low-shear endothelial microfilament bundle formation is attenuated and makes the endothelial junctions more leaky. The higher residence time of (atherogenic) particles toghether with the more leaky endothelial junctions at regions of low shear stress makes them more prone for initiation of atherosclerosis, especially in atherogenic conditions such as hypercholesterolaemia (280). In general, the coronary arteries may be extensively involved by atherosclerosis, while arteries with less complex unidirectional flow pattern, such as the renal and mesenteric vessels, tend to be spared in regions beyond their ostia (281-282). If cyclic reversal in flow direction favors plaque formation and such oscillations occur mainly during systole - coronary arteries should be expected to be especially vulnerable, for in the course of the cardiac cycle, coronary arteries are subjected to two prominent changes in flow velocity during systole (283), compared with a single oscillation for other major visceral arteries. Individual differences in heart rate would therefore be expected to exert a long-term selective effect on the coronary arteries by doubling the systolic exposure to oscillations in flow direction. In addition, at a higher heart rate, diastolic period - during which flow is unidirectional and relatively increased - is shortened while systole is relatively uninfluenced by the heart rate (72). As in the case of pulsatile circumferential stress, heart rate might also be an important determinant of shear stress. The importance of heart rate in coronary artery disease has been emphasized recently. Perski et al described an association between minimum heart rate and both the extent of coronary atherosclerosis and its progression in patients after myocardial infarction (284). High shear forces induce platelet aggregation in vitro as well as in animal models of coronary artery thrombosis (285). In man, iears in the vascular wall can occur at sites with high shear, especially adjacent to ectatic calcified lesions (79).

From these studies it can be hypothesized that, with an intact endothelium, high shear promotes endothelial integrity and function and protects the vessel against atherogenesis. However, if the endothelium is impaired or lost, high shear stress 
might be harmful and could promote atherogenesis or acute coronary syndromes. Endothelial dysfunction, like disturbed endothelial-dependent vasodilation, has been shown in different conditions such as atheromatosis, hypercholesterolaemia, ischaemia, and hypertension (286).

In hypertension shear stress is decreased (287), which might make the endothelium more prone to atherogenesis. Data on pharmacological modulation of shear stress are scarce. The B-blocker atenolol does not change shear stress whereas carteolol increases shear stress (267).

It seems too early to get a clear understanding of the impact of changes in shear stress on ill effects of hypertension. Nowadays shear stress cannot be measured accurately in man. Much progress in the understanding of the effect of shear stress on the vessel wail might be made, when new techniques, which can measure shear stress more accurately, will be available. These techniques are being developed.

\section{3. conclusion}

Pulsatile stress plays an important role in the genesis of the complications of hypertension. Vessel wall properties have a central fole as determinanis of pulsatile stress factors and can be improved by several (but not all) classes of antihypeitensive drugs. Improvement of vessel wall properties of large arteries seems a logical strategy in treatment of hypertension. However, long term prospective studies are needed correlating different aspects of vessel wall stress with morbidity and mortality in hypertension. Other important issues for future research are reversal of structural vessel wall changes, effects on local wall tension, determination of the relative impact of different stress factors of the pulsatile stress and impact of shear stress. 


\section{chapter 2. the effect of verapamil on carotid artery distensibility and cross-sectional compliance in hypertensive patients ${ }^{2}$}

\section{introduction}

Distensibility and compliance are vessel wall properties of large arteries, which are decreased in hypertension. Distensibility reflects the elasticity of the artery and is a determinant of the stress on the vessel wall. Compliance reflects the buffering capacity of large arteries and is an important determinant of the afterload on the heart. This and also the effect of anthypertensive therapy have been reviewed in the previous chapter.

The aim of the present prospective study was to investigate the effect of oral, chronic verapamil treatment on the distensibility and cross-sectional compliance of the common carotid artery in hypertensive subjects following a double-blind, crossover protocol. A calcium-blocker was chosen because this type of antihypertensive drug has a relaxing effect on smooth muscle cells (288). The common carotid artery was used in this study because it is easily accessible to ultrasound, while it is known to be more distensible (average relative diameter increase in systole of $9.6 \%$ (255) to $14.2 \%$ (289)) than, for instance, the femoral artery (relative diameter increase in systole of about $3 \%(290)$ ). The relative diameter changes of the common carotid antery during the cardiac cycle were recorded on-line with a high resolution multigate pulsed Doppler system (291). This system also allows the on-line recording of velocity profiles $(255,292-294)$. From the width of these profiles, absolute internal diameters can be determined. Arterial pulse pressure was estimated from brachial artery cuff blood pressure measurements and arterial distensibility and cross-sectional compliance were calculated as previously described in detail (295).

\section{materials and methods}

The study was performed on 19 patients with essential hypertension (10 males and 9 temales) with diastolic blood pressures $>90 \mathrm{mmHg}$, but $<120 \mathrm{mmHg}$. Nine patients were younger than 40 years and 10 were 60 years and older. After a washout period of at least 4 weeks, in which all antihypertensive drugs and diuretics were withdrawn, the patients entered a double-blind, randomized, placebocontrolled, crossover study. Patients were given verapamil tablets (120 mg) or apparently identical placebo tablets three times daily for 4 weeks. This period was followed by another 4 -week crossover period. In the 4 th week of each doubleblind period, blood pressure and heart rate were measured, and ultrasound investigation of the common carotid artery was performed. The age of the patients varied from 21 to 73 years (mean of 49 years) and their weight averaged $82 \mathrm{~kg}$ (range of $52-103 \mathrm{~kg}$ ). They all had normal serum protein and albumin levels, and normal liver function tests. Serum creatinine levels were $<134 \mu \mathrm{mol} / /$. None of them suffered from symptomatic atherosclerotic disease.

2 based on: T Van Merode, L Van Bortel, FAM Smeets, R Böhm, J Mooij, KH Rahn and RS Reneman. The effect of verapamil on carotid artery distensibility and cross-sectional compliance in hypertensive patients. J Cardiovasc Pharmacol 1990, 15:109-113. 
The ultrasound investigations were performed 2 hours after the last dose. The subjects, were in the supine position with the head tilted slightly to the contralateral side. Both the right and left common carotid artery were examined. The relative diameter changes $(\Delta \mathrm{d} / \mathrm{d} \times 100 \%)$ of the common carotid artery during the cardiac cycle were recorded on-line with a multigate pulsed Doppler system (Fig 2.1), the characteristics of which have been described in detail before $(255,291-292)$. The diameter changes were recorded in the plane of the carotid artery bifurcation. The assessment of vessel wall displacement is based upon the processing of low frequency Doppler signals, originating from the sample volumes coinciding with the anterior and posterior walls. The Doppler signals originating from the walls are 30-100 times higher in amplitude than the signals originating from the slowly moving blood cells close to the vessel wall and, hence, mask the signals induced by these cells completely. The small size of the sample volume $\left(1.2 \mathrm{~mm}^{4}\right.$ at a depth of $15 \mathrm{~mm}$ ) excludes contamination with other slowly moving structures. To insure that the initial relative change at the beginning of the cardiac cycles is constant, it is rese to zero by a trigger derived from the $\mathrm{R}$ wave of a standard lead of the ECG. The relative diameter changes can be detemined with an absolute accuracy of $0.5 \%$ (291), comparing favorably to the peak excursions observed. This means that, for a relative excursion of, for instance, $7.0 \%$, a relative change in diameter between 6.5 and $7.5 \%$ can be measured. The multigate pulsed Doppler system also allows the on-line recording of velocity profiles in arteries, i.e., the velocity distribution of the cross-section area of the vessed, at discrete time intervals during the cardiac cycle (292-294). From the width of the velocity profiles, the systolic diameter of the artery can be assessed (Fig 2.1) tather accurately (205) with an error of about $0.7 \mathrm{~mm}$. Since the diameter, as determined in this way, is depend. ent on the angle of interrogation, the values measured were corrected for this angle, i.e. $60^{\circ}$ in the present study.

During the ultrasound measurements, systolic and diastolic blood pressure and heart rate were measured in the supine position with a semi-automated device (Dinamap). With this technique, the arterial pulse pressure (systolic minus diastolic blood pressure) can be measured adequately (296). The systolic and diastolic pressures were recorded at least 6 times during the Doppler investigation, and the average value of the last 4 recordings was taken as the patient's reading. The pulse pressure was assessed on the right arm and the left arm, and the average value of these two readings was used for further calculations. This was allowed because no significant difference between the average blood pressure values of left and right arm was found.

The peak systolic value of $\Delta \mathrm{d} / \mathrm{d} \times 100 \%$, the absolute diastolic diameter (d) and the pulse pressure $(\Delta p)$ were used to calculate the distensibility coefficient (DC) and the cross-sectional compliance (CC) as previously described (295) with the use of the following equations:

$\mathrm{DC}=2 \Delta \mathrm{d} / \mathrm{d} / \Delta \mathrm{p}$

$\mathrm{CC}=\Delta \mathrm{d} / \mathrm{d} / 2 \Delta \mathrm{p} \times \pi \mathrm{d}^{2}$

Differences between the values in the placebo and verapamil periods were evaluated for statistical significance according to the non parametric Koch procedure for crossover design (297). In this procedure, the possible effects of treatment, interaction, and order of administration were tested for significance.

A p-value of $<0.05$ was considered to indicate a statistically significant difference. 


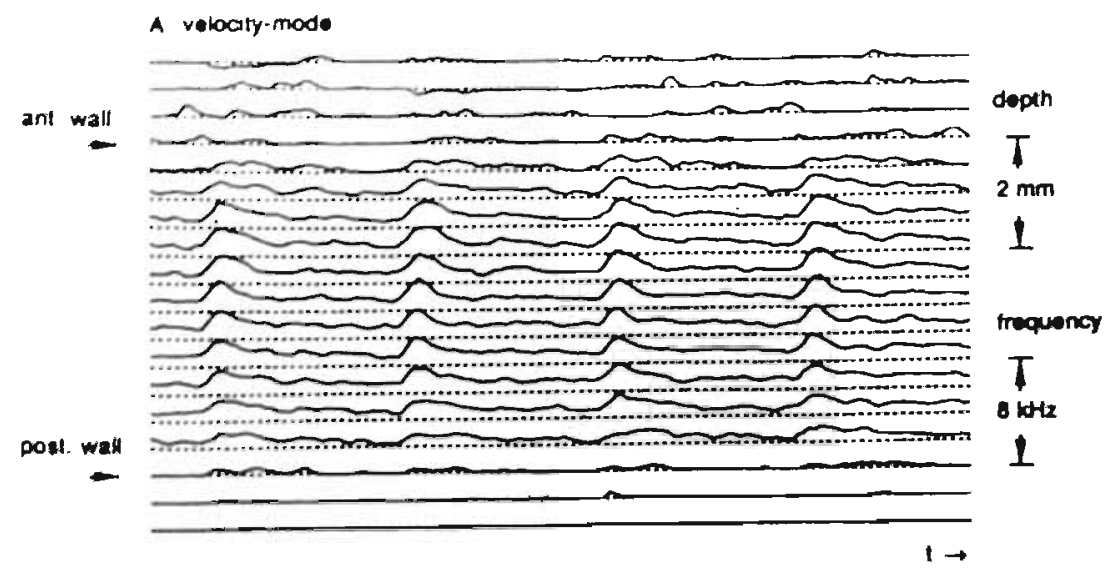

B relative voseel wall movemem

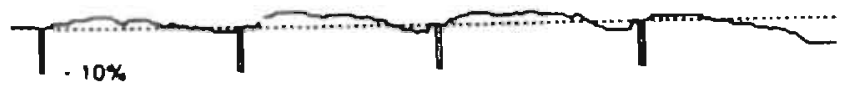

C prolite-made

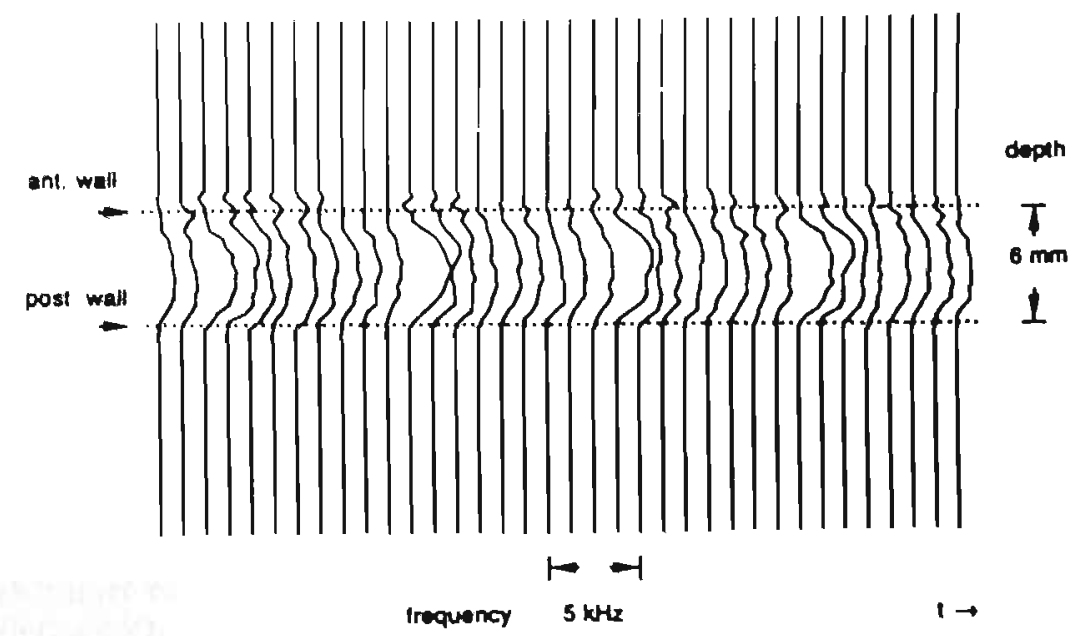

Fig. 2. 1 The instantaneous mean velocity wave forms (velocity mode: $A$ ), relative vessel wall movements (B), and axial velocity profiles (profile mode, $C$ ), as recorded' in the common carotid artery of a patient aged 48 years. Only the first three relative diameter macings were taken to assess the patients' reading. The last tracing is an artifact due to transducer movement. The dotted lines indicate the width of the profiles during systole from which the arterial diameter was estimated. 


\section{results}

On average, mean systolic and diastolic blood pressures were higher during placebo than during verapamil treatment (153/95 vs. $147 / 92 \mathrm{mmHg}$ ). There were no significant effects of interaction and order of administration. The results of treatment are depicted in Table 2.3.

Table 2.3. Arterial diameter (d), relative diameter changes $(\Delta d / d)$. distensibility $(D C)$. cross-sectional compliance $(C C)$, and pulse pressure $(\Delta P)$ for the right $(R)$ and left (L) common carotid artery during terapamil and placebo periods

\begin{tabular}{|c|c|c|c|}
\hline & & Placebo & Verapamil \\
\hline \multirow[t]{2}{*}{ Diameter (mm) } & $k$ & $7.2 \pm 0.2$ & $7.5 \pm 0.2$ \\
\hline & L. & $7.3 \pm 0.2$ & $7.2 \pm 0.2$ \\
\hline \multirow[t]{2}{*}{$\Delta \mathrm{d} / \mathrm{d}(\%)$} & $\mathrm{R}$ & $4.5 \pm 0.5$ & $5.3 \pm 0.4^{4}$ \\
\hline & L. & $4.4 \pm 0.4$ & $54 \pm 0+4$ \\
\hline \multirow[t]{2}{*}{$\operatorname{DC}\left(10^{-3} / \mathrm{kPa}\right)$} & R & $10.7 \pm 1.1$ & $13.1+1.0^{3}$ \\
\hline & L. & $10.5 \pm 1.0$ & $13.3 \pm 1.07$ \\
\hline \multirow[t]{2}{*}{$\mathrm{CC}\left(\mathrm{mm}^{2} / \mathrm{kPa}\right)$} & $\mathrm{R}$ & $0.34 \pm 0.04$ & $0.43 \pm 0.034$ \\
\hline & $\mathrm{I}$ & $0.34 \pm 0.04$ & $0.40 \pm 0.03$ \\
\hline$\Delta p(\mathrm{mml} / \mathrm{g})$ & & $64 \pm 2$ & $61 \pm 2$ \\
\hline
\end{tabular}

"statistically significant difference between placebo and verapamil; data are mean \pm SEM.

The relative change in artery diameter during the cardiac cycle was significantly higher during verapamil treatment than during the placebo period, for both the left and the right common carotid artery $(p<0.02$ and $p<0.05$, respectively). The distensibility coefficient (DC) was significantly increased during verapamil treatment, as compared to the placebo period for both left and right common carotid artery $(p<0.01$ and $p<0.05$, respectively). The cross-sectional compliance (CC) was increased during verapamil treatment, but reached the level of significance. only for the right common carotid artery $(\mathrm{p}<0.05)$. The p-value for the left common carotid artery was 0.16 . The common carotid artery diameter and the arterial pulse pressure were not significantly different during verapamil and placebo treatment.

Changes in blood pressure, heart rate and vessel wall properties were also examined in the subgroups of young (21-40 years) and elderly (60-73 years) patients (Table 2.4). In elderly patients, blood pressure was higher and vessel wall properties lower. During verapamil the decrease in blood pressure was more pronounced in elderly patients, while the increase in distensibility and compliance was higher in young patients. 
Table 2.4. Haemodynamic effects of verapamil after 4 weeks $3 x 120 \mathrm{mg}$ per day in young and elderly bypertensive patients.

\begin{tabular}{|c|c|c|c|c|}
\hline & \multicolumn{2}{|c|}{ young $(\mathbf{n}=9)$} & \multicolumn{2}{|c|}{ elderly $(\mathrm{n}=10)$} \\
\hline & placebos & verapanil & placebo & verapamil \\
\hline SBP (mmHg) & $148 \pm 4$ & $140 \pm 4^{2}$ & $159 \pm 5$ & $146 \pm 4^{2 i}$ \\
\hline DBP (mumllg) & $87 \pm 3$ & $80 \pm 32$ & $92 \pm 2$ & $83 \pm 2^{b}$ \\
\hline$\Delta \mathrm{P}$ (mmHg) & $61 \pm 2$ & $61 \pm 3$ & $67 \pm 3$ & $63 \pm 3$ \\
\hline $\operatorname{HR}(\mathrm{b} / \mathrm{min})$ & $74 \pm 5$ & $70 \pm 4$ & $76 \pm 3$ & $70 \pm 3^{b}$ \\
\hline b $(\mathrm{mm})$ & $69 \pm 0.3$ & $7.3 \pm 0.4$ & $7.5 \pm 0.2$ & $7.8 \pm 0.3$ \\
\hline$D C\left(10^{4} / \mathrm{KP} .1\right)$ & $13.1 \pm 1.6$ & $16.5 \pm 1.2$ & $8.5 \pm 1.1$ & $10.0 \pm 0.8$ \\
\hline$C C\left(\mathrm{~nm}^{2} / \mathrm{kPa}\right)$ & $0.38 \pm 0.06$ & $0.51 \pm 0.04$ & $0.30 \pm 0.05$ & $0.35 \pm 0.04$ \\
\hline
\end{tabular}

SBP: systolic blood pressure; DBP: diastolic blood pressure; $\Delta \mathrm{P}$ : pulse pressure; HR: heart rate; D: diameter; DC: distensibility cocfficient; CC: Compliance coeflecient Data are presented as mean \pm SEM; ${ }^{2} \mathrm{p}<0.05,{ }^{\mathrm{b}} \mathrm{p}<0.01 \mathrm{statistical}$ difference versus placebo.

\section{discussion}

The findings in the present study indicate that chronic treatment with the calciumantagonist verapamil increases distensibility and cross-sectional compliance of the common carotid artery in hyperiensive patients. These improvements have to be considered as changes in arterial wall distensibility because no significant differences in arterial diameter could be detected between treatment with the compound and placebo. The latter observation is interesting because verapamil apparently improves arterial wall distensibility without dilating the artery significantly.

That the improvement of cross-sectional compliance during verapamil treatment did not reach the level of significance for the left carotid artery is probably caused by the slightly, but non-significantly, smaller mean carotid artery diameter (maybe due to a stray value) on that side, especially since the distensibility is significantly improved. One should keep in mind that slight differences in diameter do have a great impact on cross-sectional compliance, because of the second power of this measure in the equation to calculate this parameter (see equation 2).

The improvement of the reduced vessel wall distensibility in hypertensive patients through chronic treatment with verapamil could result in a better management of the systolic flow jet from the heart, and may help to protect the patient against atherosclerotic complications of hypertension.

The diminished arterial wall distensibility, which means a stiffer behavior, in young hypertensive patients indicates that in these patients the arteries age faster (298). Therefore, antihypertensive treatment should not only aim at lowering arterial blood pressure, but also at improving the distensibility of the arteries. In this context, it should be kept in mind that improved arterial wall compliance during anti- 
hypertensive treatment may be achieved through arterial dilation without an effect on arterial wall distensibility. Although through this compensating mechanism arteries are able to better store volume energy, it remains to be seen whether dilation alone prevents the arteries from early aging. Further studies are required to assess the true value of these findings.

As expected blood pressure was higher in older patients and distensibility and compliance of the common carotid artery (CCA) were smaller. Despite the larger decrease in systolic blood pressure and pulse pressure during verapamil treatment in eldery patients, the change in vessel wall properties was smaller. This difference can hardly be explained by the difference in blood pressure alone, since in elderly patients blood pressure during verapamil was even lower than during placebo in young patients, but distensibility remained lower in the elderly. These data are compatible with a stiffer large vessel in the elderly and strongly suggest structural changes in the CCA of the elderly. These results suggest that verapamil given for 1 month has less effect on arterial distensibility and compliance when structural changes are present

A criticism of the method, as used in the present study, could be that pulse pressure was measured at the brachial rather than the common carotid anery. This was necessary because a non-invasive method to measure pressure in the latter artery is not available. In this approach, it is assumed that the pulse pressure in the brachial artery is representative of that in the common carotid antery. An indication of this assumption is the positive relationship between this pulse pressure and the relative diameter increase of the common carotid artery during systole, as found in a previous study (295), examining age-related changes in distensibility and crosssectional compliance in a similar way. However, early reflected pulse waves can occur especially in elderly patients. These pulse wave reflections can raise the pulse pressure in the ascending aorta and probably also in the carotid artery. The pulse pressure in the brachial artery is in general not influenced by pulse wave reflections $(169,299)$. It cannot be excluded that in the elderly patients of the present study the pulse pressure in the carotid artery is influenced by early reflected pulse waves. If this is the case, then compared to the younger patients pulse pressure would be higher and the vessel wall properties lower than measured. As a result the difference in distensibility and compliance between young and elderly patients would even be more pronounced.

In conclusion, the results of this study indicate that verapamil administration in hypertensive patients increases both carotid artery distensibility and crosssectional compliance. Although increased compliance probably compensates for a decrease in arterial elasticity, it does not necessarily protect the arteries against aging faster in hypertensive patients. The improvement in distensibility, as found with chronic treatment with verapamil in this study, could be even more important in this respect. 


\section{chapter 3. effect of nebivolol on distensibility and compliance of the common carotid artery ${ }^{3}$}

\section{introduction}

In hypertension large arteries are aging faster and atherosclerosis, a major cause of morbidity and mortality, is developing earlier than in normotensive patients (300). Therefore, the goals of modern antihypertensive treatment will be not only normalization of blood pressure and preservation of quality of life, but also prevention of atherosclerotic disease (125).

Not only in patients with essential hypertension (301) but also in borderline hypertensive patients (298) compliance of large arteries is diminished. Since stift arteries manage the systolic pressure pulse from the heart less adequately, endothelial injury and plaque rupture may occur more frequently in these vessels. Thus, improvement of the vessel wall properties of large vessels might protect them from atherosclerotic disease. The influence of physical factors on large vessels has been discussed in chapter 1 .

In this study the effect of nebivolol on vessel wall properties (distensibility and compliance) of the common carotid artery are investigated with a high resolution multigate pulsed Doppler system. Nebivolol is a selective $B_{1}$-adrenoceptor antagonist with vasodilating properties (302). Five $\mathrm{mg}$ once daily has on average a good antihypertensive eftect (303-304).

\section{materials and methods}

After a 4-week single blind run-in period, during which all antihypertensive drugs and diuretics were withdrawn, 29 patients (21 males, 8 females; aged $25-70$ yr) entered the study.

The study was a double-blind, randomized, placebo controlled crossover study. The study design is shown in Fig 2.2. In each crossover period, patients were given $5 \mathrm{mg}$ nebivolol or apparently identical placebo once daily for 4 weeks. At the end of each 4:week period, arterial blood pressure, heart rate and vessel wall properties were measured. Measurements were performed at a fixed time of the day, which was for each patient a fixed time after daily dose intake as well.

All patients suffered from essential hypertension and were recruited from outpatient clinics. None suffered from symptomatic atherosclerotic disease. Diastolic blood pressure was $>90 \mathrm{mmHg}$ but $<120 \mathrm{mmHg}$ at the end of the 4 -week washout period. All patients had normal liver function tests and normal serum protein and albumin levels. Serum creatinine levels were $<134 \mu \mathrm{mol} /$. The study was approved by the Medical Ethical Committee of the University of Limburg. Ail patients gave their written informed consent.

\footnotetext{
3 based or:

- LMAB Van Bortel, T Van Merode, FAM Smeets, RS Reneman, JMV Mooij, HAJ Struyker Boudier. Nebivolal improves the vessel wall properties of the common carotid artery. Drug Invest 1991 , 3(suppl 1):61-63.

- T Van Merode, LM Van Bortel, FA Smeets, RS Reneman, R Bōhm, KH Rahn. Verapamil and nebivolol improve carotid artery distensibility in hypertensive patients. J Hypertens 1989, 7(suppl 6): 262-263.
} 


\section{Study design}

\begin{tabular}{|c|c|c|}
\hline single-blind & double-blind & double-blind \\
\hline \multirow{2}{*}{ Run-in } & Nebivolol & Placebo \\
\hline & Placebo & Nebivolol \\
\hline
\end{tabular}

Fig. 2.2. Study design: a 4-week single blind placebo rum-in period was followed by 2 4-week double blind crossover periods (nebivolol, placebo).

Vessel wall properties of the common carotid artery were measured with a multigate pulsed Doppler system, as described previously (292). The relative change in diameter of the right common carotid artery during the heart cycle was recorded on-line. From the width of the on-line recorded velocity profiles in the artery, the diameter of the common carotid artery can be measured. The relative change in diameter can be recorded with an absolute accuracy of $0.5 \%$ (292). The diameter, as determined with this technique, is dependent on the angle of interrogation. Therefore, the values measured, were corrected for this angle, $60^{\circ}$ in the present study.

Simultaneously with the Doppler recordings of the common carotid artery, arterial blood pressure and heart rate were measured with a semi-automated device (Dinamap, Critikon, Tampa, USA). Blood pressure and heart rate were recorded at least 6 times during Doppler investigation. The average value of the last four recordings was taken as the patient's reading. Pulse pressure was calculated from the average values of the readings on the right and left arm. All measurements of vessel wall properties and arterial blood pressure were performed in supine position after 15 minutes of rest.

From the diameter (D), the relative change in diameter during the heart cycle $(\Delta D / D)$ and the pulse pressure $(\Delta P)$, distensibility coefficient (DC) and crosssectional compliance coefficient (CC) were calculated:

formula: $\mathrm{DC}=2(\Delta \mathrm{D} / \mathrm{D}) / \Delta \mathrm{P}$

formula: $C C=(\Delta D / D) \cdot \pi D^{2} / 2 \Delta P$

Statistical analysis was performed according to the non-parametric Koch procedure for the two-way crossover design (297), which allows testing for treatment, period and interaction effects. A p-value $<0.05$ was considered a significant difference. 


\section{results}

All patients completed the study. No serious adverse events occurred. No statistically significant interaction or period effects were seen between the two crossover periods. Data on haemodynamics are shown in Table 2.5 .

Table 2.5. Haemodynamic effects of 4 weeks nebivoln $5 \mathrm{mg}$ once daily

\begin{tabular}{|l|c|c|}
\hline & $\mathrm{P}$ & $\mathrm{N}$ \\
\hline $\mathrm{SBP}(\mathrm{mmHg})$ & $155 \pm 3$ & $145 \pm 3 \cdots$ \\
\hline $\mathrm{DBP}(\mathrm{mmH})$ & $97 \pm 2$ & $90 \pm 2 \cdots$ \\
\hline$\Delta \mathrm{P}(\mathrm{mmHg})$ & $62 \pm 2$ & $62 \pm 2$ \\
\hline $\mathrm{HR}(\mathrm{b} / \mathrm{min})$ & $80 \pm 3$ & $69 \pm 2 \cdots$ \\
\hline
\end{tabular}

P: placebo; N: nebivolol; SBP: systolic blood pressure; DBP: diastolic blood pressure; $\triangle \mathrm{P}$ : pulse pressure; HR: heart rate; $\cdots p<0.001$ statistical difference berween placebo and nebivolol; Data are presented as mean $\pm \mathrm{SEM}$.

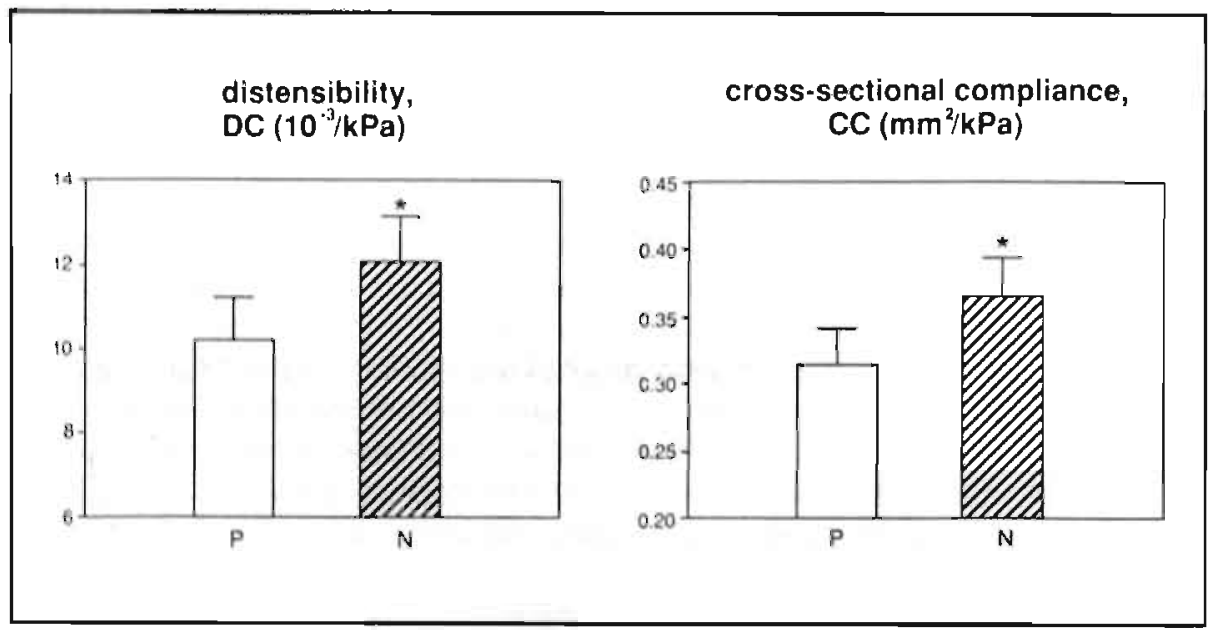

Fig 2.3. Distensibility coefficient (DC) and cross-sectional compliance (CC) of the common carotid artery during placebo $(P)$ and nebivolol $(N)$. Data are mean $\pm S E M . * p<0.05$, difference between double blind placebo and nebivolol.

Nebivolol decreased heart rate, systolic and diastolic blcod pressure $(p<0.001)$. Pulse pressure did not differ between placebo and nebivolol.

The diameter of the common carotid artery did not differ between placebo $(7.4 \pm 0.2 \mathrm{~mm})$ and nebivolol $(7.5 \pm 0.3 \mathrm{~mm})$. The effect of nebivolol on the distensibility coefficient and cross-sectional compliance of the common carotid artery is shown in Figure 2.3. With placebo, distensibility of the common carotid artery was $10.2 \pm 1.1 \quad 10^{-3} / \mathrm{kPa}$ and compliance was $0.31 \pm 0.03 \mathrm{~mm}^{2} / \mathrm{kPa}$. These two vessel wall properties increased $(p<0.05)$ during nebivolol treatment (distensibility : $12.1 \pm 1.1 \quad 10^{-3} / \mathrm{kPa}$; compliance: $0.37 \pm 0.03 \mathrm{~mm}^{2} / \mathrm{kPa}$ ). 


\section{discussion}

Distensibility and compliance are vessel wall properties of large arteries. Accurate non-invasive measurements of these vessel wall properties can be obtained by means of recently developed echo-Doppler techniques. In this study a first generation device developed by Dr Hocks and coworkers was used. The technique, its accuracy and limitations have been discussed in chapter 2.

Nebivolol is an antihypertensive drug with B-blocking and vasodilazing properties. As expected blood pressure and heart rate were decreased after 4 weeks treament with nebivolol.

The results of the present study also indicate that nebivolol favorably influences distensibility and cross-sectional compliance of the common carotid artery. The improved compliance was the result of an increased distensibility, without change in diameter of the common carotid artery. These findings might result in a better management of the systolic pressure pulse. The increased distensibility, the lower systolic blood pressure and heart rate might decrease the load on the vesset wall and as a consequence help fo protect the common carotid artery (and perhaps also other large arteries) from accelerated aging and atherosclerotic disease. The increased compliance might result in a smaller afterload for the heart, and as a consequence help to prevent cardiac hypertrophy.

As discussed in chapter 1, large artery compliance is improved by is-blocking drugs with vasodilating properties (26-268) like nebivolol and dilevalol and also by those with intrinsic sympathomimetic activity like pindolol. Non-selective B-blockers without ISA like propranolol (264) did not improve compliance, while selective $B_{1}$-adrenoceptor antagonists like bisoprolol (271) and atenolol (260) improved compliance, but metoprolol (270) did not maybe because of loss of selectivity due to a higher dose.

In conclusion, in patients with essential hypertension, $5 \mathrm{mg}$ nebivolol once daily had a good antihypertensive effect and improved vessel wall properties of the common carotid artery. This might help to prevent or delay cardiovascular disease in these hypertensive patients. 



\section{PART III \\ QUALITY OF LIFE PERCEPTION DURING ANTIHYPERTENSIVE TREATMENT}

\section{introduction}

Effective antihypertensive therapy is important since the risk of cardiovascular complications related. to hypertension increases continuously with increasing levels of both systolic and diastolic blood pressure (125). Effective antihyper. tensive therapy is influenced by both the prescribed antihypertensive drug and patient's drug adherence. Different classes of antihypenensive drugs are now available that can effect a comparable reduction in blood pressure. In contrast, patient's drug adherence is in general poor, as described in part 1 . Efforts should be made to improve it. In this respect quality of life perception cluring antihypertensive treatment is important. Since patients with mild-to-moderate hypertension are in general asymptomatic (186), therapy is not likely to improve functional status and sense of well-being, but can cause new symptoms and result in poorer adherence to therapy if the unwanted effects are substantial. As a result, it is not only important to control blood pressure but also to preserve the patient's quality of life (305-306).

Standardized information on quality of life has proven to be an important research tool in the development of drugs with equal therapeutic benefit (307-308). A quality of life measurement instrument must be reproducible and must have been validated. In addition, it has to detect clinically important changes; in other words, it has to be responsive (309). Information on quality of life can be obtained by general health profiles or disease-specific measures. General health profiles can identify aspects affected by the disease and its management. However, general measures may fail to examine adequately detailed aspects of treatment for specific symptoms or functions related to a disease (305). Choosing a good questionnaire is important, but avoiding systematic bias in questionnaire administration may even be more important. Inconsistencies in administration can be avoided by using trained interviewers or by using traditional self-administered questionnaires (309).

The Inventory of Subjective Health is a general health profile, which was originally developed by Dirken for detecting stress in the work place. Seven of its core items are identical to the items in the somatic subscale of the "John Hopkins Symptom Checklist" (HSCL). As it proved to be a sensitive instrument for health problems, it has been validated (310-311) and used in the last decade in numerous Dutch surveys - also in hypertension (312) - to indicate subjective health status. This health profile measures subjective physical well-being, also the physical well-being influenced by psychological or emotional factors. It consists of a selfadministered questionnaire containing 47 yes/no questions about self-experienced health. The lower the score, the better the subjective perception of well-being. Since the items of the Inventory of Subjective Health are mutually dependent, classical multivariate techniques are not appropriate for analysis (313).

In physically active patients, exercise tolerance may be an important determinant of quality of life. During normal and recreational physical activity maximal work capacity will usually not be reached. In contrast, prolonged exercise at a sub- 
maximal exercise level is regularly achieved. Therefore, endurance performance at a submaximal level rather than the maximal exercise capacity is the major exerciserelated determinant of patient's quality of life. A reduction in endurance capacity during is-adrenoceptor blockade has been well documented (314-320).

Chapter 1 investigates the effect on quality of life - as measured with the Inventory of Subjective Health (ISH) - of placebo and antihypertensive treatment with nebivolol, a $B_{1}$-adrenoceptor antagonist with an ancillary property. The ISH-score is compared with the results of perceived health rating and side effect diaries.

Since ACE-inhibitors are assumed to preserve quality of life better than other antihypertensive drugs, chapter 2 investigates the effect on quality of life of the ACEinhibitor enalapril compared with the effect of the selective $B_{1}$-adrenoceptor antagonist bisoprolol.

Chapter 3 investigates exercise tolerance with 2 different B-blocking agents. The effect of different antihypertensive drugs on exercise tolerance is also discussed.

\section{Appendix to introduction The Inventory of Subjective Health}

The items of the inventory of Subjective ilealth (circle yes or no).

\begin{tabular}{|c|c|c|c|}
\hline 1. & Are you regularly bothered by coughing? & yes & no \\
\hline 2. & Do you often have pains in the chest or heart region? & yes & no \\
\hline 3. & Do you regularly have unpleasant cold fingers, hands or feet? & yes & no \\
\hline 1. & Is your appetite less good than you consider normal? & yes & no \\
\hline 5. & Does your stomach often feel full and bloated? & yes & no \\
\hline 6 & Do you get short of breatl casily? & yes & no \\
\hline 7. & Do you often have an unpleasant or sweetish taste in your mouth? & yes & no \\
\hline 8. & Are you often bothered by pricking of watering eyes? & yes & no \\
\hline 9. & Are you bothered by roaring in the ears? & yes & no \\
\hline 10. & Do you feel fit lately? & yes & no \\
\hline 11. & Do you often have to clear your throat? & yes & no \\
\hline 12. & Do you often have sneezing fits? & yes & no \\
\hline 13. & Do you often feel hungry? & yes & no \\
\hline 14. & Do you often feel tight in the chest? & yes & no \\
\hline 15. & Do your bones or muscles ever ache? & yes & no \\
\hline 16 & Do you generally move your bowels every day? & yes & no \\
\hline 17. & Do you often feel tired? & yes & no \\
\hline 18. & Do you sometimes sweat heavily even when it is not hot? & yes & no \\
\hline
\end{tabular}




\begin{tabular}{|c|c|c|c|}
\hline 19. & Are you often bothered by itching? & yes & no \\
\hline 20 & Are you often bothered by headaches? & yes & no \\
\hline 21. & Do you often feel dizzy? & yes & no \\
\hline 22. & Do you often have indigestion? & yes & no \\
\hline 23. & Do you often feel slecpy or sluggish? & yes & no \\
\hline 24. & Do your arms and legs often go dead or tingle? & yes & no \\
\hline 25. & Do you often get upset? & yes & $\mathrm{no}$ \\
\hline 26. & Do you think you are too thin? & yes & no \\
\hline 27. & Do you think you are overweight? & yes & no \\
\hline 28. & Do you often feel listless? & yes & no \\
\hline 29. & Are you accident prone? & yes & no \\
\hline 30. & $\begin{array}{l}\text { Does alcohol effect you more than it used to? (if you never drink } \\
\text { alcoholic beverages put a circle around 'no') }\end{array}$ & yes & no \\
\hline 31. & Are you often irritable? & yes & no \\
\hline 32. & Do you feel tired now and then at the end of a strenuous day? & yes & no \\
\hline 33. & Do your hands ofien shake? & yes & no \\
\hline 34. & $\begin{array}{l}\text { When you get home after work, do you fall asleep in your chair right } \\
\text { away? }\end{array}$ & yes & no \\
\hline 35. & $\begin{array}{l}\text { Do you ofien have palpitations of the heart or throbbing in your } \\
\text { heart region? }\end{array}$ & yes & no \\
\hline 36. & Da you think you suffer from excessive thirst? & yes & no \\
\hline 37. & Do yous often have pains in the stomach region? & yes & nes \\
\hline 38. & Do you often have pains in or around the eyes? & yes & no \\
\hline 39. & Is your nose often blocked? & yes & no \\
\hline 40. & Do you fall asleep easily and do you sleep well? & yes & no \\
\hline 41. & Are you bothered by weak ot aching feet? & yes & no \\
\hline 42. & Are you often bothered by acne or boils? & yes & no \\
\hline 43. & Do you often feel nervous? & yes & no \\
\hline 44. & Do you generally get up feeling tired and not rested in the morning? & yes & no \\
\hline 45. & Do you often have stomach trouble? & yes & no \\
\hline 46. & Are you often troubled by backache? & yes & no \\
\hline 47. & Are you often bothered by sleeplessness? & yes & ก0 \\
\hline
\end{tabular}




\section{chapter 1. Effect of placebo and antihypertensive treatment with nebivolol on haemodynamics and quality of life as measured with the Inventory of Subjective Health ${ }^{1}$}

\section{introduction}

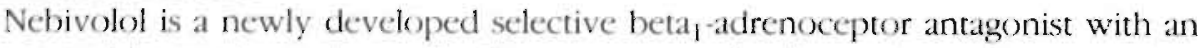
unusual haemodynamic profile. In contrast to other beta-adrenergic antagonists, nebivolol acutely lowered arterial blood pressure in spontaneously hypertensive rats. In healthy volunteers, nebivolol $(5 \mathrm{mg})$ lowered systemic vascular resistance during daily oral treatment and did not impair left ventricular function (302). This suggests the presence of a pharmacologic ancillary vasodilating property, apart from the beta $a_{1}$ adrenoceptor antagonism. The nature of this ancillary property is still unknown. In vitro and in vivo studies did not show alpha-, serotoninz, calcium-antagonism, nor a direct vasodilating action. There was also no evidence for intrinsic sympathomimetic activity (302). In hypertensive patients, the antihypertensive effect of nebivolol $5 \mathrm{mg}$ once claily was greater than that of $2.5 \mathrm{mg}$ once daily. Ten mg/day did not enhance the antihypertensive effect (303-304).

This study investigates the antihypertensive efficacy, adverse effects and influence on quality of life perception of a 4 and 8 -week treatment with nebivolol $5 \mathrm{mg}$ once daily,

\section{materials and methods}

Pationts

1.31 patients were recruited from outpatient clinics. At the end of a 4 -week single blind placebo run-in period 10 patients did not meet the blood pressure entry criteria and 7 were excluded for other reasons. 114 patients with essential hypertension were selected and entered the study. Demographic data are shown in Table 3.1. Inclusion criteria were a diastolic blood pressure between $95 \mathrm{mmHg}$ and $120 \mathrm{mmHg}$ or a diastolic blood pressure between 90 and $94 \mathrm{mmHg}$ with a systolic blood pressure of more than $160 \mathrm{mmHg}$ at the end of the 4-week run-in per̃iod. Serum levels of ALAT and ASAT did not exceed rwice the upper limit of normal. Serum levels of creatinine were lower than $190 \mu \mathrm{mol} / 1$. Fifty six patients regularly consumed 2 glasses ( $<1$ up to 10 ) alcohol per day. Twenty nine patients smoked 11 (1-30) cigarettes per day. Fifty-two of 114 patients were receiving other non-antihypertensive medications during double blind treatment. At baseline, 3 patients showed clear ECG signs of left ventricular hypertrophy with strain. Five patients had ECG parameters compatible with an old inferior wall and 2 with an old anterior wall infarction. Three patients had ECG signs of ischaemia of the inferior wall. Two complete right bundle branch block and 1 ECG with atrial fibrillation were seen.

I based on: LMAB Van Bortel, JGS Breed, J Joosten, JA Kragten, FAT Lustermans, JMV Mooij. Nebivolol in hypertension: a double blind placebo-controlled multicenter study assessing its antihypertensive efficacy and impact on quality of life. J Cardiovasc Pharmacol 1993, 21:856-862. 
Table 3.1. Demograpbic data $(n=114)$

\begin{tabular}{|l|l|}
\hline Male/female & $76 / 38$ \\
\hline Race & $\begin{array}{l}\text { Caucasian } 112 \\
\text { Black I } \\
\text { Asian I }\end{array}$ \\
\hline Age (years) & $54 \pm 1(25-77)$ \\
\hline Height (cm) & $169 \pm 1(150-191)$ \\
\hline Weight $(\mathrm{kg})$ & $78 \pm 1(48-127)$ \\
\hline
\end{tabular}

Data are mean $\pm S E M$ and (range)

\section{Study design}

The study design is shown in Figure 3.1. After a 4-week single blind placebo run-in period, during which all antihypertensive drugs wefe withdrawn, 114 patients entered a double blind placebo-controlled multicenter study. Major exclusion criteria were a systolic blood pressure of more than $200 \mathrm{mmHg}$ and a diastolic pressure of more than $120 \mathrm{mmHg}$, a heart rate lower than 50 beats/min, atrioventricular block (second and third degree), signs of heart failure, clinically relevant impaired renal and liver function, uncontrolled diabetes, severe vascular disease, clinically relevant signs of bronchospasm, a history of cerebrovascular accident or myocardial infarction within 3 months preceding the study, poor general condition or life expectancy, lack of compliance or pregnancy.

\begin{tabular}{|c|c|c|c|}
\hline single-blind & double-blind & double-blind & \multirow{5}{*}{ group $1(n=40)$} \\
\hline \multirow{3}{*}{$\begin{array}{l}\text { Placebo } \\
\text { Run-in }\end{array}$} & Nebivolol $5 \mathrm{mg}$ & Placebo & \\
\hline & Placebo & Nebivolol $5 \mathrm{mg}$ & \\
\hline & Nebivolol $5 \mathrm{mg}$ & Nebivolol $5 \mathrm{mg}$ & \\
\hline 4 weeks & 4 weeks & 4 weeks & \\
\hline \multicolumn{2}{|c|}{ Randomization } & & \\
\hline
\end{tabular}

Fig 3.1. Study design: A 4-week single blind placebo run-in period was followed by 2 4-week double blind periods: nebivolol-placebo, placebo-nebivolol, or nebivolol-nebivolol.

Selected patients were randomized into 3 groups and were given nebivolol $5 \mathrm{mg}$ solution $(5 \mathrm{ml}$ ) or placebo identical in appearance. Group 1 consisted of 40 patients and received nebivolol in the first double blind 4-week period followed by placebo 
in the second 4 -weck period. Group 2 consisted of 40 patients and was given placebo followed by nebivolol. Group 3 was given nebivolol for 8 weeks.

At the end of each 4 -week period supine blood pressure and heart rate were measured, a 12-lead electrocardiogram (ECG) was recorded and blood was taken for determination of hacmatologic and biochemical parameters. All measurements were performed at a fixed time of the day, which was for each patient a fixed time after daily dose intake as well. Time between dose intake and measurements varied between patients from 3 to 10 hours. At the same visit a quality of life questionnaire was filled out. In addition, patients were asked to rate their perceived health. During week 3 and 4 of each 4 -week period, prevalence and duration of side effects were assessed with a diary.

Volunteers were asked about their compliance. Volunteers' compliance was also checked by assessing the amount of drug retumed. The study was approved by the ethical committee of each participating center. All patients gave their written or witnessed oral informed consent.

\section{Methods}

Blood pressure and heart rate were measured after 15 minutes of supine rest and after 2 minutes standing. Data are means of 3 consecutive measurements in each position. Heart rate was measured by counting radial pulse during 30 seconds. Blood pressure was measured with a sphygmomanometer at the right arm and preferably by the same investigator. Diastolic blood pressure was defined at Korotkoff $V$. Response rates were calculated from patients in groups 1 and 2 $(n=68)$ with supine diastolic blood pressure at randomization (baseline) of $95 \mathrm{mmHg}$ or more. Patients were considered as responders if diastolic blood pressure during clouble blind treatment was normalized ( $\leqq 90 \mathrm{mmHg}$ ) or if diastolic blood pressure was decreased with at least $10 \%$.

Quality of life was measured with the Inventory of Subjective Health (ISH). This self-administered questionmaire contains 47 questions about common complaints that may be present or absent for some time. The ISH score is a valid indicator of subjective health status $(310-311)$. The lower the score, the higher the perception of well-being.

Perceived health was rated on a 5 -point scale from very $\operatorname{good}(=1)$ to very badl $(=5)$. This is a valid predictor of mortality over a 10-year period (321).

Adverse effects were assessed with a diary. Each day I page had ro be filled in. Each page contained questions about common symptoms in hypertension, including those known to be related to beta-blocker therapy. If a complaint was not listed it could be added to the list.

ECG parameters were calculated from an electrocardiogram recorded at $50 \mathrm{~mm} / \mathrm{sec}$. Parameters were means of at least 5 heart cycles. QT-time was corrected for heart rate by the $\mathrm{QT}_{\mathrm{C}}$ formula of Bazen (322): $\mathrm{Q}^{\circ} \mathrm{T}_{\mathrm{c}}=\mathrm{Q}^{\mathrm{T}} / \sqrt{\mathrm{RR}}$.

At the end of the visit, non-fasting blood samples were taken for determination of haematologic and biochemical parameters. Haematologic parameters consisted of red and white blood ceil counts, platelet count, haemoglobin, haematocrit and 1-hour red blood cell sedimentation rate (ESR). Biochemical parameters were serum levels of sodium, potassium, calcium, total protein, albumin, uric acid, urea, creatinine, total bilirubin, ASAT, ALAT, GGT, alkaline phosphatase, total cholesterol and glucose. 


\section{Statistical methods}

All data are presented as mean \pm S.E.M. Data after 4 weeks of nebivolol and double blind placebo were analysed in groups 1 and $2(\mathrm{n}=80)$, which form a crossover design. Statistical analysis was performed according to the non-parametric Koch procedure for the two-way crossover design (297) using the Mann-Whitney U-test. Comparative data analysis within group 3. $(n=32)$ was made by the Wilcoxon m.p.s.r. test. Haemodynamic data after 8 weeks of treatment with nebivolol (group 3) were also compared with those after 4 weeks of treatment with nebivolol (group $2 ; n=40$ ), which, for both groups, comprises 8 weeks after initial randomization. Statistical analysis of this parallel design was performed with the MannWhitney U-test. Comparison with baseline was made with the Wilcoxon m.p.s.r. test when appropriate. Baseline was defined as the end of the 4 -week placebo run-in period, which was at randomization. A p $=$ value $<0.05$ was considered a. significant difference.

\section{results}

At the end of each 4 -week period, patients returned the drugs left and were asked about their drug compliance. From patients' answers about their drug adherence (mean $98 \%$; range $91-100 \%$ of prescribed dose taken) and from the assessment of the amount of drug returned, patients' compliance was estimated as more than $90 \%$. Two patients dropped out. Both were randomized to group 3. One patient dropped out because of recurrent peptic ulcer. The second patient withdrew con. sent. The weight of patients did not change throughout the study.

Four weeks of treatment with nebivolol

Four weeks treatment with nebivolol was compared with 4 wecks double blind placebo in a crossover design (groups 1 \& 2). No statistically significant carry-over effects were seen between the two crossover periods. Except for supine systolic blood pressure, also no period effects were present.

Data on blood pressure and heart rate are shown in Fig 3.2

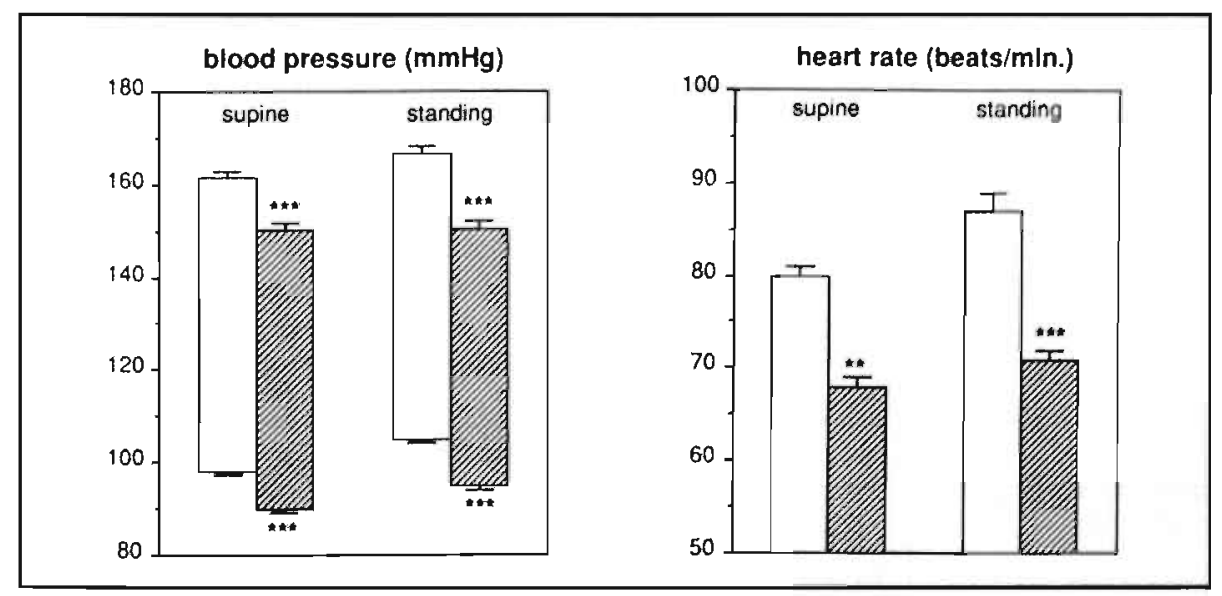

Fig 3.2. Supine and standing systolic blood pressure, diastolic blood pressure and beart rate after 4 weeks treatment with (ש) nebivolol versus double blind

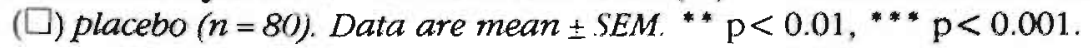


During nebivolol treatment, statistically significant decreases $(p<0.0001)$ in systolic and diastolic blood pressure as well as in heart rate were seen. In the supine position, blood pressure decreased from $161 \pm 2 / 98 \pm 1 \mathrm{mmHg}$ during placebo to $150 \pm 2 / 90 \pm 1 \mathrm{mmHg}$ during nebivolol, and heart rate from $80 \pm 1$ to $68 \pm 1 \mathrm{~b} / \mathrm{min}$. In standing position blood pressure was $166 \pm 2 / 105 \pm 1 \mathrm{mmHg}$ during placebo and decreased to $150 \pm 2 / 95 \pm 1 \mathrm{mmHg}$ during nebivolol. Heart rate was $87 \pm 2$ $\mathrm{b} /$ min during placebo and $71 \pm 1 \mathrm{~b} / \mathrm{min}$ during nebivolol. Blood pressure response rate to a 4-week treatment with nebivolol $5 \mathrm{mg}$ once daily was $65 \%$ (Fig 3.3). Response rate to placebo was $25 \%$.

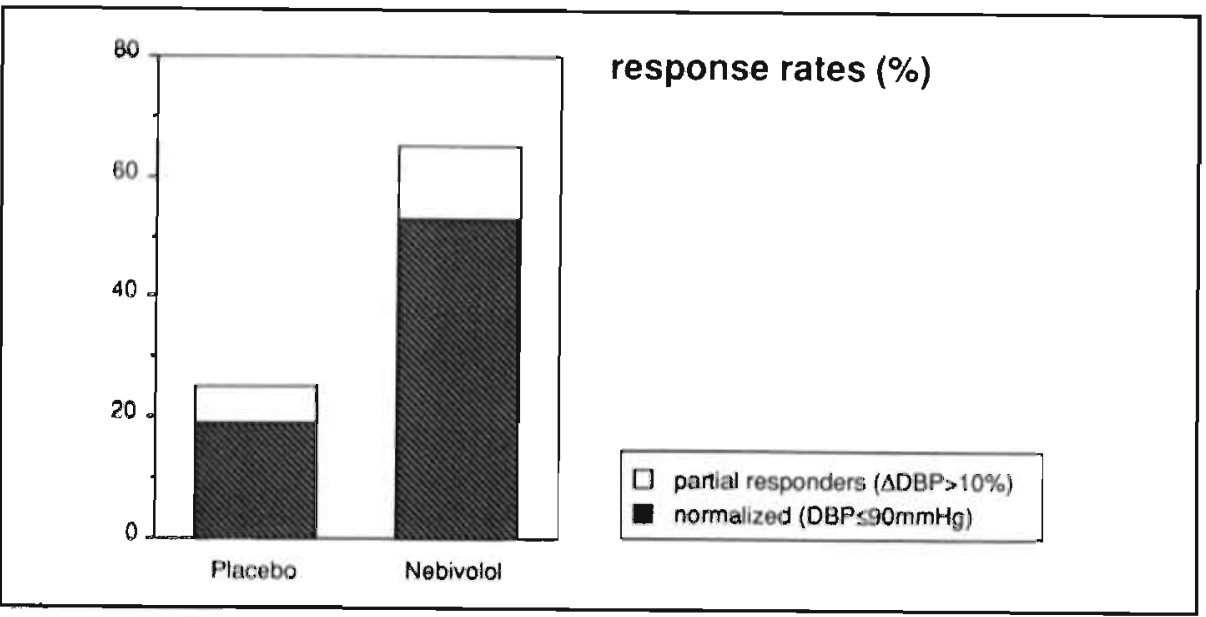

Fig. 3.3. Blood pressure response rates (\%) after 4 weeks treatment $(n=68)$

ECG parameters are shown in Table 3.2. The PQ interval increased with nebivolol and QRS-time remained unchanged. As a result of the reduction in heart rate QTinterval increased. QT-time corrected for heart rate showed a shortening of $\mathrm{QT}_{\mathrm{C}}$ during nebivolol. Two ECG's showing left ventricular hypertrophy with strain at baseline changed during double blind treatment: strain disappeared in one instance during nebivolol and in the other instance during placebo following the nebivolol treatment sequence. One right bundle branch block disappeared during nebivolol and was again present during the consecutive placebo period. The other ECG's remained unchanged in shape throughout the study.

Table 3.2. ECG parameters at baseline and after 4 weeks of treatment (groups I and 2).

\begin{tabular}{|l|l|l|l|}
\hline & Baseline & Placebo & Nebivolol \\
\hline PQ (ms) & $168 \pm 3$ & $169 \pm 3$ & $175 \pm 3^{* *}$ \\
\hline QRS (ms) & $86 \pm 2$ & $86 \pm 2$ & $86 \pm 2$ \\
\hline QT (ms) & $366 \pm 3$ & $367 \pm 3$ & $390 \pm 4^{* \cdots}$ \\
\hline $\mathrm{QT}_{\mathrm{c}}(\mathrm{ms})$ & $409 \pm 3$ & $412 \pm 3$ & $398 \pm 3^{*}$ \\
\hline
\end{tabular}

" $\mathrm{p}<0.05 ; \cdots$ p $\cdots 0.001$ double blind placebo versus nebivolol 
Quality of life perception - as measured with the Inventory of Subjective Health was evaluable in 79 patients. The ISH score was $11.2 \pm 0.8$ at the end of the run-in period and decreased $(\mathrm{p}<0.001)$ to $9.5 \pm 0.7$ during double blind placebo and nebivolol (Fig 3.4). Perceived health rated on a 5-point scale $(n=79)$ was $2.32 \pm 0.07$ at the end of the run-in period (Fig 3.4). During placebo perceived health was $2.25 \pm 0.06$ and did not differ $(p>0.5)$ during nebivolol treatment $(2.23 \pm 0.06)$. Baseline values did not differ significantly from the 2 double blind periods.

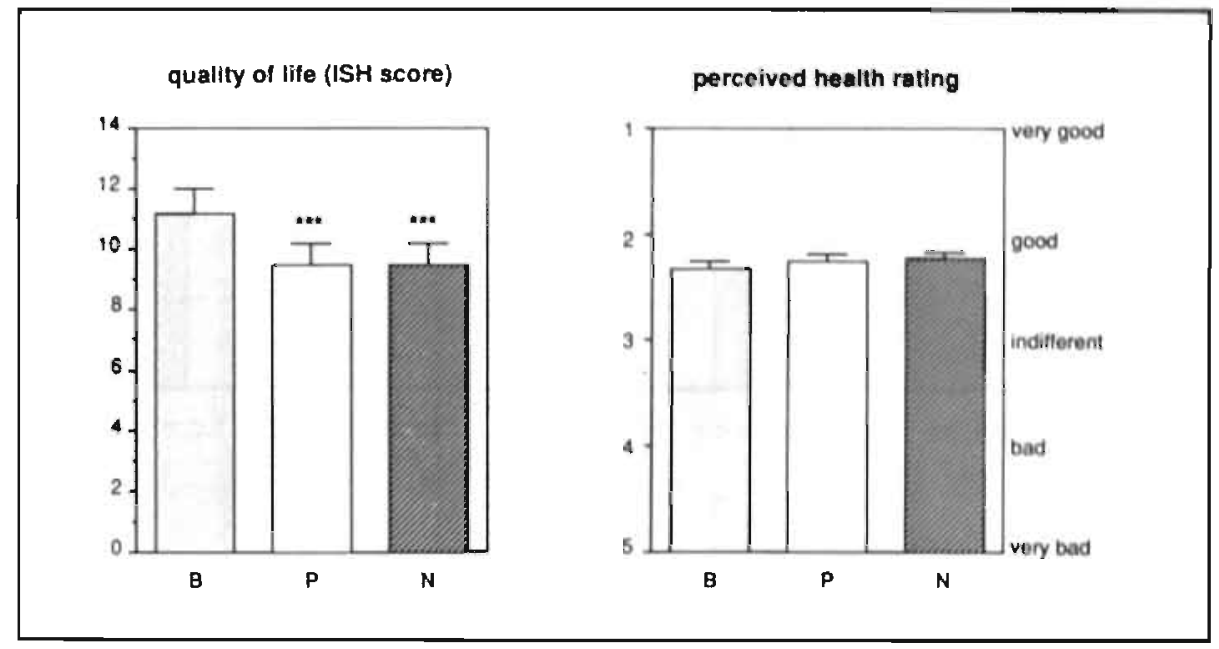

Fig 3.4. ISH score and perceived health rating after 4 weeks treatment (nebivolol and placebo) and at baseline $(n=79)$. The lower the score, the better the subjective well-being and perceived bealth. Data are mean \pm SEM. B(baseline); $P$ (placebo); $N($ nebivolol). ** $p<0.001$ (difference versus baseline).

Adverse effects were recorded in a diary. Diaries of 79 (all but 1) paticnts, who filled in at least 7 days, were analysed. Complaints were rated for presence in days per 100 patient days. Most relevant out of 65 complaints are shown in Fig 3.5. Only 3 complaints were statistically different between placebo and nebivolol. Headache decreased $(p<0.01)$ from 15.0 with double blind placebo to 9.4 days $/ 100$ patient days with nebivolol. Difficulty falling asleep (sleep problems) decreased $(\mathrm{p}<0.02)$ from 13.3 to 10.8 days $/ 100$ patient days during nebivolol treatment. Complaints of dry mouth also decreased statistically during nebivolol. Although often a trend was seen, other complaints did not differ statistically between nebivolol and placebo. The total number of complaints during double blind placebo was $333 \mathrm{com}$ plaints $/ 100$ patient days and 312 complaints $/ 100$ patient days during nebivolol. The difference was not statistically significant.

Table 3.3 lists the most relevant laboratory data after 4 weeks treatment with double blind placebo and nebivolol. In addition, it shows the number of patients with laboratory values within the normal range at baseline but with abnormal values during double blind placebo and nebivolol, respectively. For the laboratory data, which are not listed, this number of patients did not differ with more than 1 between placebo and nebivolol. 


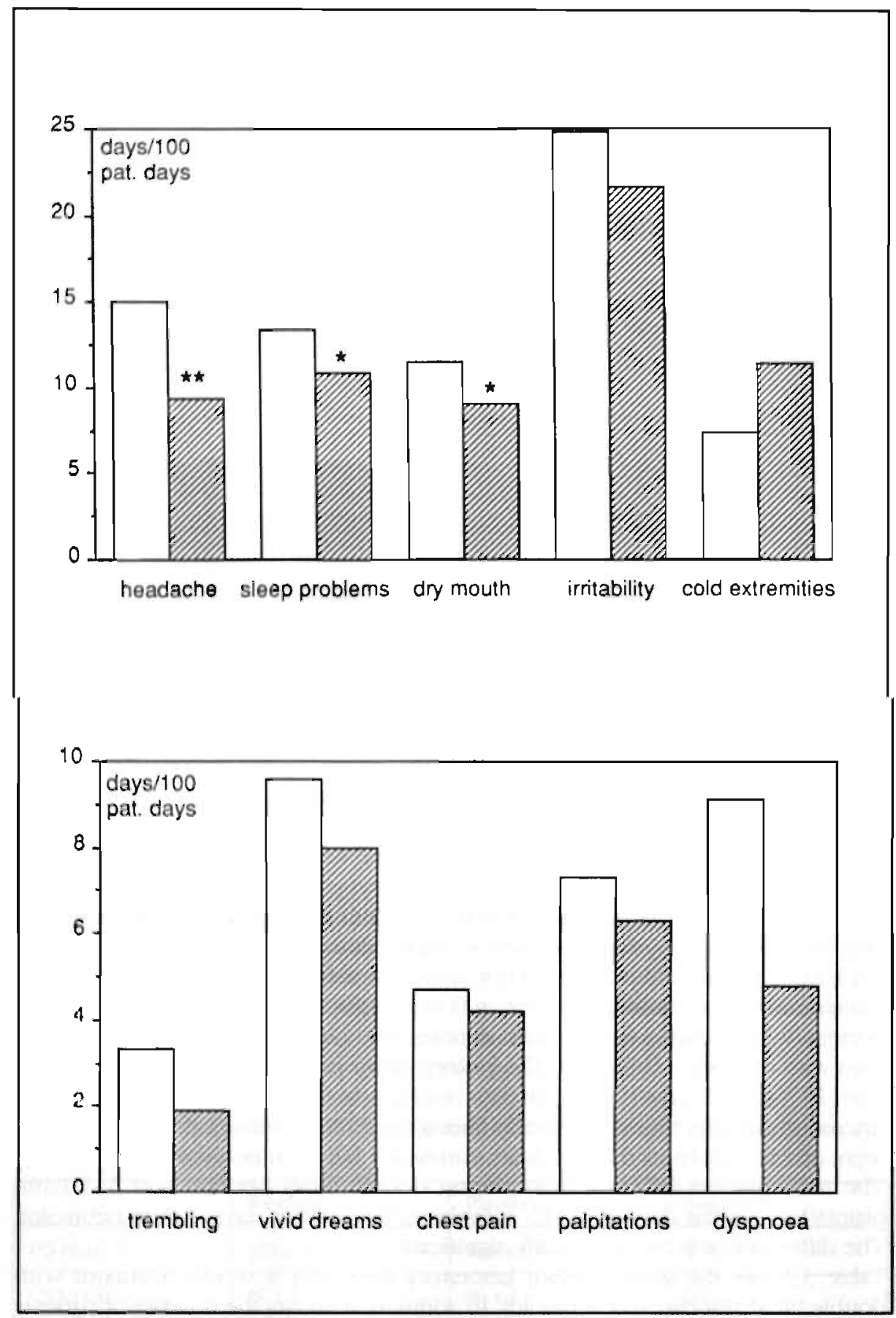

Fig 3.5. Adverse effects after 4 weeks nebivolol (1) versus placebo ( $\square$ ): most relev ant out of 65 complaints. Data are means. " $p<0.05,{ }^{* *} p<0.01$. "Vivid dreams" is the sum of 2 complaints: many dreams and nightmares; "Irritability" is the sum of nervousness and rapidly irritated. 
Table 3.3. Laboratory data after 4 weeks of treatment (groups I and 2).

\begin{tabular}{|c|c|c|c|c|c|c|}
\hline & \multicolumn{3}{|c|}{ Mean value $\pm S E M$} & \multicolumn{3}{|c|}{ Changes from baseline } \\
\hline & Bascline: & Placebo & Nebivo[ol & Change & $\begin{array}{l}\text { Placebo } \\
\text { (n) }\end{array}$ & $\begin{array}{l}\text { Nebivolol } \\
\text { (ni) }\end{array}$ \\
\hline ESR (mm) & $12 \pm 1$ & $11 \pm 1$ & $11 \pm 1$ & $\uparrow$ & 10 & 8 \\
\hline $\begin{array}{l}\text { Platelets } \\
\text { (giga/l) }\end{array}$ & $255 \pm 13$ & $246 \pm 12$ & $239 \pm 14^{\cdots}$ & $\downarrow$ & 0 & u \\
\hline $\begin{array}{l}\text { Calcium } \\
\text { (mmol/h) }\end{array}$ & $2.33 \pm 0.02$ & $2.35 \pm 0.01$ & $2.35 \pm 0.01$ & $\uparrow$ & 3 & 1 \\
\hline $\begin{array}{l}\text { Glucose } \\
(\mathrm{mmol} / \mathrm{)})\end{array}$ & $5.8 \pm 0.3$ & $5.8 \pm 0.3$ & $5.6 \pm 0.2$ & 1 & 4 & 5 \\
\hline $\begin{array}{l}\text { Total } \\
\text { cholesterol } \\
(\mathrm{mmol} / \mathrm{)})\end{array}$ & $6.4 \pm 0.1$ & $6.3 \pm 0.1$ & $6.3 \pm 0.1$ & 1 & 4 & 7 \\
\hline $\begin{array}{l}\text { Alkaline } \\
\text { phosphatase } \\
(U / I)\end{array}$ & $86 \pm 3$ & $83 \pm 3$ & $82 \pm 3$ & $\dagger$ & 1 & 3 \\
\hline GGT (U/I) & $33 \pm 3$ & $34 \pm 3$ & $37 \pm 4$ & $\dagger$ & 2 & 2 \\
\hline ALAT (U/l) & $18 \pm 2$ & $17 \pm 1$ & $19 \pm 3$ & $\uparrow$ & 2 & 2 \\
\hline Urea (mmol/l) & $6.1 \pm 0.2$ & $6.1 \pm 0.2$ & $6.5 \pm 0.2^{* *}$ & $\uparrow$ & 3 & 3 \\
\hline $\begin{array}{l}\text { Creatinine } \\
\text { ( } \mathrm{moll} \text { ) }\end{array}$ & $92 \pm 3$ & $95 \pm 3$ & $96 \pm 3$ & $\dagger$ & 2 & 1 \\
\hline $\begin{array}{l}\text { Uric acid } \\
(\mu \mathrm{mol} / \mathrm{l})\end{array}$ & $320 \pm 12$ & $327 \pm 11$ & $344 \pm 13^{\circ}$ & $\uparrow$ & 1 & 1 \\
\hline $\begin{array}{l}\text { Potassium } \\
\text { (mmol/1) }\end{array}$ & $4.2 \pm 0.1$ & $4.3 \pm 0.1$ & $4.7 \pm 0.2^{*}$ & $\begin{array}{l}4 \\
1\end{array}$ & 5 & $\begin{array}{l}6 \\
2\end{array}$ \\
\hline
\end{tabular}

statistical difference versus placebo ${ }^{*} p<0.05 ;{ }^{\cdots} p<0.01 ;(n)$ Number of patients with value within normal range at baseline and abnormal value during doụble-blind placebo or nebivolol. 
Eight weeks of treatment with nebivolol

Eight weeks treatment with nebivolol are compared with 4 weeks in group 3. Data on blood pressure, heart rate, quality of life and ECG are shown in Table 3.4. Shape of ECG's remained unchanged throughout the study.

Table 3.4. Data after 4 and 8 weeks nebivolol (group 3).

\begin{tabular}{|c|c|c|c|}
\hline hacmodynamics & baseline & I weeks & 8 weeks \\
\hline supine SBP $(\mathrm{mmHg})$ & $165 \pm 3$ & $148 \pm 3$ & $142 \pm 3 "$ \\
\hline supine DBP ( $\mathrm{mml} / \mathrm{g})$ & $101 \pm 1$ & $91 \pm 2$ & $87 \pm 2, \cdots$ \\
\hline supine IIR (b/min) & $82 \pm 2$ & $68 \pm 2$ & $69 \pm 2$ \\
\hline standing SBP (mmH/g) & $165 \pm 3$ & $147 \pm 4$ & $144 \pm 4$ \\
\hline standling DBP ( $\mathrm{mmH}$ H) & $106 \pm 1$ & $93 \pm 2$ & $92 \pm 2$ \\
\hline standing HR (b/min) & $88 \pm 2$ & $71 \pm 2$ & $72 \pm 2$ \\
\hline \multicolumn{4}{|c|}{ inventory of subjective health } \\
\hline score & $13.1 \pm 1.6$ & $11.3 \pm 1.6$ & $11.5 \pm 1.5$ \\
\hline \multicolumn{4}{|l|}{ perceived health rating } \\
\hline score & $2.43 \pm 0.12$ & $2.32 \pm 0.10$ & $2.31 \pm 0.09$ \\
\hline \multicolumn{4}{|l|}{ electrocardiogram } \\
\hline$P Q$ (msec) & $170 \pm 4$ & $175 \pm 4$ & $174 \pm 4$ \\
\hline QRS (msec) & $87 \pm 2$ & $88 \pm 2$ & $89 \pm 2$ \\
\hline QT (msec) & $367 \pm 6$ & $394 \pm 4$ & $394 \pm 5$ \\
\hline QTe (msec) & $420 \pm 5$ & $406 \pm 4$ & $404 \pm 4$ \\
\hline
\end{tabular}

SBP: sysiolic blood pressure; DBP: diastolic blood pressure; HR : heart rate; differences between week 4 and $8:{ }^{*} p<0.05 ; \cdots p<0.01$. Data are mean \pm SEM.

Heart rate and standing blood pressure did not differ between 4 and 8 weeks treatment with nebivolol. The decrease in supine blood pressure was larger after 8 weeks than after 4 weeks treatment. This could, at least in part, be due to a hidden placebo effect. Therefore, results after 8 weeks nebivolol (group 3) were compared with those after 4 weeks nebivolol (group 2). In group 2, supine blood pressure was $165 \pm 3 / 101 \pm 1 \mathrm{mmHg}$ and heart nate was $78 \pm 2 \mathrm{~b} / \mathrm{min}$. In the standing position these values were $167 \pm 3 / 107 \pm 1 \mathrm{mmHg}$ and $82 \pm 2 \mathrm{~b} / \mathrm{min}$, respectively. Decreases from these baseline values are shown in Fig 3.6. Supine and standing blood pressures and heart rates tended to decrease somewhat less after 4 than after 8 weeks of nebivolol. The differences between week 4 and 8 were not statistically significant.

Diaries for side effects of 31 patients (all but 1 ), who filled in at least 7 days, were analysed. The number of patients with complaints did not differ between week 4 and 8 of treatment with nebivolol, but was lower than at baseline.

No clinically relevant differences in laboratory values were seen between week 4 and week 8 . The number of patients - with a normal laboratory value at baseline and abnormal value during the double blind periods - did not differ with more than 1 berween week 4 and week 8 . 


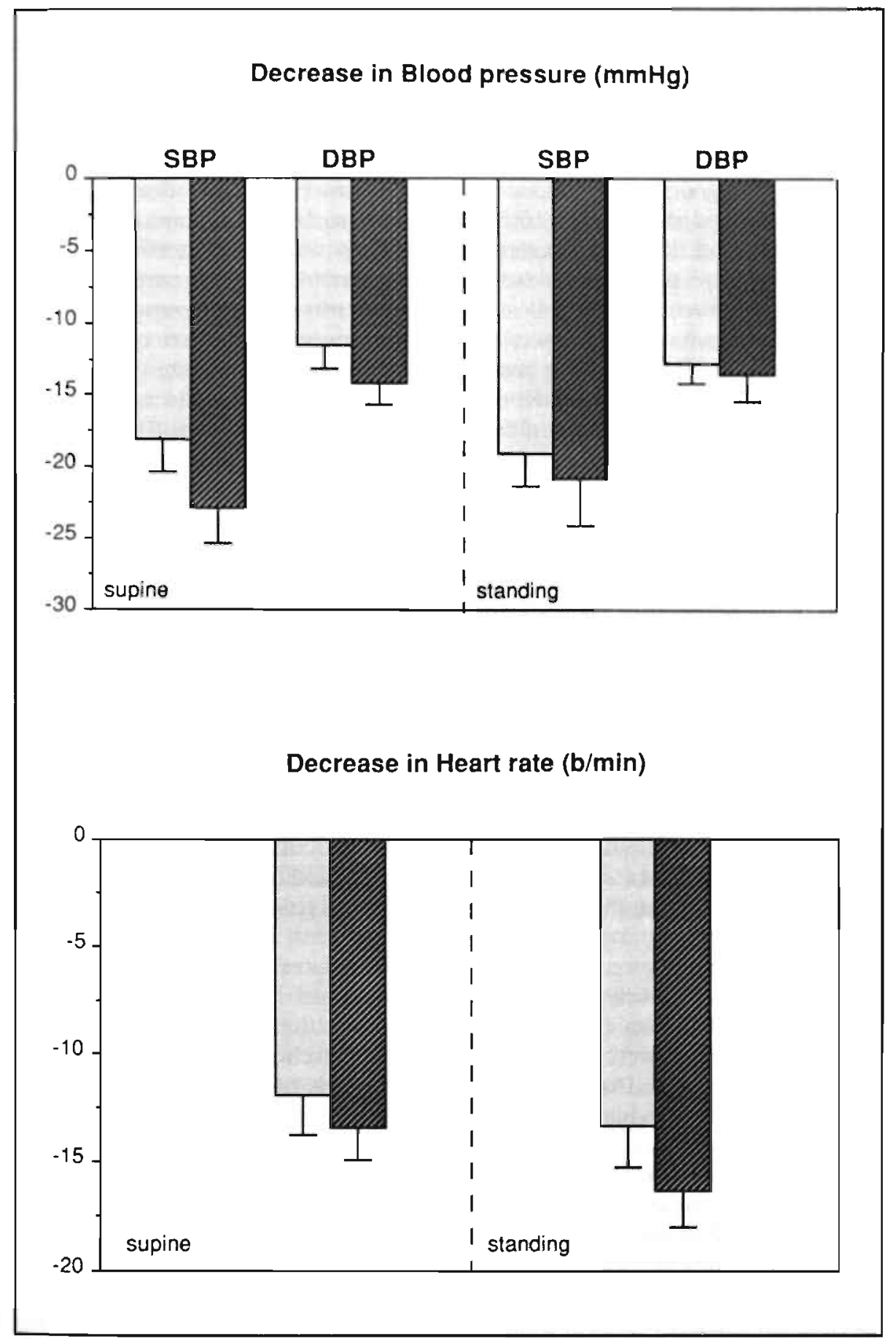

Fig 3.6. Decrease in supine and standing systolic blood pressure, diastolic blood

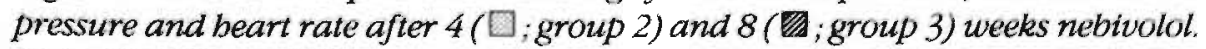
Data are mean $\pm S E M$. 


\section{discussion}

Nebivolol is an antihypertensive drug with beta-blocking properties. As a result resting supine and standing heart rate decreased during treatment with nebivolol. The decrease in heart rate was not larger after 8 than after 4 weeks of nebivolol. Although heart rate at rest is also largely detemined by vagal rone, these data suggest that steady state beta-blocking effect was reached within 4 weeks.

Nebivolol $5 \mathrm{mg}$ once daily induced a good antihypertensive effect in supine (10/8 $\mathrm{mmHg}$ ) and standing (16/10 $\mathrm{mmHg}$ ) position. After 2 minutes standing no signs of orthostatic hypotension were present. Response rate to placebo was $25 \%$. liesponse rate to $5 \mathrm{mg}$ ncbivolol was $65 \%$. Comparable response rates have been described for placebo (323-324) and antihypertensive monotherapy (32+326). A fixed close of nebivolol was used in the present study in contrast to other studies investigating response rates. The antihypertensive effect in the supine position was higher after 8 than after 4 weeks of treatment with nebivolol. In addition, the standing blood pressure did not differ berween week 4 and week 8 . This indicates absence of drug tolerance over the 8 -week period. Could the larger antihypertensive effect (in supine position) after 8 weeks be due to a period effect? Comparison of week 8 in group 3 with week 8 in group 2 (4 weeks placebo followed by 4 weeks nebivolol) can correct for a possible hidden period effect. Differences in antihypertensive eflect at week 8 between group 2 and 3 were not statistically significant. It secms reasonable to suggest that a period effect might be present. ECG changes during nebivolol are those associated with other beta-blocking agents (327-328). PQ-time increased during nebivolol treatment, but no atrioventricular block of the second or third degree occurred. QRS-time was not changed. QT-time increased, but heart rate corrected QT-time (QT ) was shorter than during placebo. Changes in the electrocardiogram did not differ between week 4 and 8 with nebivolol.

After a 4-week treatment period, no clinically important changes in mean values of laboratory data were found between nebivolol and double blind placebo. Similar to other beta-blockers (329-330) blood urea, uric acid and potassium increased slightly with nebivolol. Platelets showed a slight decrease. But for each of these 4 laboratory items, similarly few patients had a normal value at bascline and an abnormal value during treatment with nebivolol or placebo. As a consequence, the differences are not likely to be of clinical relevance. Liver enzymes and total cholesterol showed no clinically relevant changes during nebivolol. Since no fasted blood samples were taken, serum levels of HDL cholesterol and triglycerides were not determined. During nebivolol serum levels of plasma glucose did not differ from the double blind placebo period and were similar after 4 and 8 weeks of treatment. It is not clear whether glucose tolerance is less impaired with nebivolol than with other beta-blocking drugs. The effects of nebivolol on blood biochemistry are in accordance with those found by Chan et al (331). In this latter placebo-controlled study with 16 patients in the nebivolol group, fasted serum triglycerides levels did not increase during nebivolol.

It is generally accepted that quality of life trials have to be large, usually 150-200 patients per treatment group, owing the variability of data collected (332). This variability in collected data is in part due to the interindividual variability in studies with a parallel groups design. Quality of life perception depends upon different conditions such as age, sex and social status (333-336). In a study with a crossover 
design, differences in overall quality of life scores berween ketanserin and placebo could be shown with only $1^{-7}$ patients $\left(33^{7}\right)$. Therefore, the crossover approach has been suggested as useful with agents that have no carry-over effect (338). In the present placebo-controlled study, no carry-over effect was seen as measured with the Koch procedure for the two-way crossover design. The ISH score did not differ between double blind placebo and nebivolol. This indicates that during a 4 week treatment with nebivolol $5 \mathrm{mg} /$ day quality of life perception was preserved. Quality of life perception improved during both nebivolol and placebo versus baseline. This improvement versus baseline might be due to the participation in the trial (339). As a consequence an absolute change in quality of life can only be demonstrated in a placebo-controlled study and not by comparison with pretreatment baseline measurements. The majority of studies investigating quality of life are not placebo-controlled but comparative (338). As a result, in those studies the only conclusion can be that one drug is better than another. Comparison with the baseline status might be misleading, and might induce incorrect conclusions. Like the ISH score, the rating of perceived health did not differ between nebivolol and double blind placebo. It also tended to be better during both placebo and nebivolol versus baseline. These data are consistent with the resuls of the ISH score. ISH scores and perceived health rating did not differ between week 4 and week 8 of treatment with nebivolol. This indicates that quality of life perception was very constant and preserved for at least 8 weeks.

Adverse effects were registered with a diary. As with other beta-blocking drugs (340-341), the prevalence of headache decreased during nebivolol. Nebivolol might be devoid of CNS adverse effects, since sleep problems occurred less frequently and vivid dreams were not increased but even tended to decrease. During nebivolol dyspnoea tended to be less frequent than during placebo. This is unusual for a beta-blocking drug, and no $\mathrm{B}_{2}$-agonistic effect of nebivolol is known, which might induce bronchodilation. The lower incidence of dyspnoea during nebivolol might in part be due to the exclusion from the study of patients with overt signs of bronchospasm. In addition, it might reflect a lower incidence of the "shortness of breath" feeling clue to hyperventilation. This hyperventilation can be improved by beta-blocker induced decrease in nervousness.

The favorable adverse effect profile, the total number of complaints, which did not differ and even tended to decrease slightly (6\%) during nebivolol, and the fact that during the study only 2 patients $(<2 \%)$ dropped out, indicate that nebivolol is well tolerated and support the results of the quality of life assessments.

In conclusion, nebivolol $5 \mathrm{mg}$ once daily is an effective antihypertensive agent, with response rates comparable to those of beta-blocker monotherapy. No signs of drug tolerance were seen. Nebivolol $5 \mathrm{mg}$ once daily has a fivorable adverse effect profile and preserves quality of life as measured with the Inventory of Subjective Health and perceived health rating. As far as the electrocardiogram and blood analyses are concemed, treatment with nebivolol $5 \mathrm{mg}$ once daily scems to be safe. Whether glucose tolerance is preserved during nebivolol needs further investigation.

The present study also indicates that an absolute change in quality of life perception cannot be claimed by comparison with baseline, since participation in a trial already influences quality of life perception. The study also supports the validity of the Inventory of Subjective Health as a measure for general well-being, since results, were in accordance with both the perceived health rating and the side effect diaries. 


\section{chapter 2. Do ACE-inhibitors preserve quality of life better than other antihypertensive drugs? A comparative study between enalapril and bisoprolol $^{2}$}

\section{introduction}

Quality of life has become a relevant measure of efficacy in clinical studies. Its use is spreading and its importance growing as a valid indicator of whether or not a medical treatment is beneficial (342).

Short-term therapy with angiotensin-converting-enzyme (ACE)-inhibitors for hypertension is effective and weil-tolerated, and compared to beta-blockers, may cause fewer adverse reactions (313). This study investigates the antihypertensive effect and influence on patients' quality of life perception of the highly selective $B_{1}$-adrenoreceptor blocker bisoprolol compared to the ACE-inhibitor enalapril.

\section{material and methods}

\section{Paticnts}

Fifty-seven patients with mild-to-moderate essential hypertension were eligible. Fifty-four patients (37 males, 17 females: 14 smokers, 40 non-smokers) were selected to enter the active treatment period. Patients were from 20 to 69 years (mean 50 years) old, their height ranged from 148 to $190 \mathrm{~cm}$ (mean $172 \mathrm{~cm}$ ) and their weight from 60 to $119 \mathrm{~kg}$ (mean $82 \mathrm{~kg}$ ). Eighteen patients were not treated with any antihypertensive drug before starting the study. The other 36 selected patients were treated for hypertension for $4.8 \pm 0.8$ years $(0-18$ years) with different drugs (Table 3.5). Ten patients were treated for concomitant diseases at study entry. Concomitant medication consisted of clofibrate, simvastatin, omeprazol, prednisolone, tryptizol, lithium carbonate, dosulepine, disopyramide, baclofen and acitretine. Three eligible patients did not enter the study. Two of them had normal blood pressure after the run-in period and 1 withdrew consent.

\section{Study design}

After an initial screening visit, cligible patients stated the study with a 2 -weck run-in period, during which all anthypertensive drugs were withdrawn. At the end of the run-in period patients were selected. Included were patients with a supine diastolic blood pressure higher than $95 \mathrm{mmHg}$ but lower than $115 \mathrm{mmHg}$. Major exclusion criteria were a systolic blood pressure higher than $200 \mathrm{mmHg}$, a heart rate lower than 50 beats/minute, an atrioventricular block of the second or third degree, signs of heart failure, diabetes, impaired renal (creatinine $>140 \mu \mathrm{mol} / \mathrm{l}$ ) or liver function or lack of compliance. Selected patients entered a multicenter double blind comparative two-way crossover study. Patients were given in random order bisoprolol (Emcor ${ }^{R}$ ) $5 \mathrm{mg}$ tablets or enalapril (Renitec ${ }^{\mathrm{R}}$ ) $10 \mathrm{mg}$ tablets once daily for 8 weeks. The first treatment period was immediately followed by the second. If after 4 weeks of each 8 -week treatment period diastolic blood

\footnotetext{
2 based on: jGS Breed, R Ciampricotti, GP Tromp, FA Valster, E Lageweg, LMAB Van Bortel. Quality of life perception during antihypertensive treatment: a comparative study between Bisoprolol and Enalapril. J Gardiovasc Pharmacol 1992, 20:750-755.
} 
pressure was higher than $90 \mathrm{mmHg}$, doses were doubled (bisoprolol $10 \mathrm{mg}$ or enalapril $20 \mathrm{mg}$ ). At the end of the run-in period and at the end of each 8-week treatment period blood pressure and heart rate were measured and a quality of life questionnaire was filled out. Patients were also asked for possible complaints. A 12-lead electrocardiogram was taken and serum levels of sodium, potassium, creatinine and glucose were determined. In each patient, measurements were performed at the same time of the day. At the end of the double blind study patients were asked with an open question to indicate the treatment period during which they judged their subjective well-being better. The study was approved by the ethical committee of each participating center. All patients gave their written or witnessed oral informed consent.

\section{Table 3.5. Antibypertensive treatment before starting the study}

\begin{tabular}{|l|l|}
\hline \multicolumn{2}{|l|}{ no antihypertensive drugs $\mathrm{n}=18$} \\
\hline antihypertensive monotherapy $\mathrm{n}=19$ & $\mathrm{n}$ \\
diuretics (1) & 4 \\
beta-adrenoreceptor antagonists (2) & 6 \\
calcium-antagonists (3) & 3 \\
ACE-inhibitors (4) & 6 \\
\hline antihypertensive double therapy $\mathrm{n}=16$ & \\
(1) $+(2)$ & 1 \\
$(1)+(3)$ & 5 \\
(1) $+(4)$ & 2 \\
$(2)+(3)$ & 4 \\
(2) + (4) & 3 \\
(3) + (4) & \\
\hline antihypertensive triplc therapy $\mathrm{n}=1(2)+(3)+(4)$ \\
\hline
\end{tabular}

\section{Methods}

Two consecutive measurements of blood pressure and heart rate were performed after at least 3 minutes of supine rest and after 2 minutes in upright position. Means of these 2 values were used for statistical analysis. Heart rate was measured by counting the radial pulse frequency and blood pressure was measured with a mercury sphygmomanometer. Diastolic blood pressure was defined at Korotkoff V. Quality of life was assessed by the Inventory of Subjective Health. This validated questionnaire consists of 47 yes/no questions (310-311). The lower the score, the higher the perception of well-being. Patients were asked to judge their subjective well-being over the last 2 weeks. Only spontaneously mentioned adverse effects were registered by the physician. Patients were not asked for any particular complaint. At the end of the observation period, patients were asked which period they preferred for continuation of their treatment. Subsequently, the treatment code was broken by the treating physician, and the drug given in the preferred period was prescribed. 


\section{Statistical methods}

Data are presented as mean \pm S.E.M. Measurements at the end of each 8 -week treament period were used for statistical comparison. Statistical analysis was performed according to the nonparametric Koch procedure for the two-way crossover design using the Mann-Whitney U test (297). Apart. from "efficacy" analysis on all patients who completed the study, an intention-to-treat analysis was performed according to the "last observation carried forward" principle (343). Data from the intention-to-treat analysis are presented. A p-value lower than 0.05 was considered as significant.

\section{results}

Nine patients did not complete the study (Table 3.6). One patient had an overt lack of compliance during bisoprolol treatment and was therefore excluded from the study and study-analysis. Eight patients dropped out because of side effects. Seven of them during treatment with enalapril and 1 during bisoprolol.

Table 3.6. Dropouts

\begin{tabular}{|c|c|c|c|}
\hline & patient number & treatment period & reason of dropout \\
\hline & (1) & 1 & withdrawal of consent \\
\hline during bisoprolol & (2) & 2 & tiredness, arrhythmia \\
\hline during enalapril & $\begin{array}{l}\text { (3) } \\
\text { (4) } \\
\text { (6) } \\
\text { (7) } \\
\text { (8) } \\
\text { (9) }\end{array}$ & $\begin{array}{l}1 \\
1 \\
1 \\
1 \\
2 \\
2 \\
2\end{array}$ & $\begin{array}{l}\text { headache, dizziness } \\
\text { cdema } \\
\text { headache, dizziness, gastric pain, } \\
\text { impotency, intolerance for alcohol } \\
\text { cough, gastric and throat pain } \\
\text { dizziness } \\
\text { dizziness, palpitations } \\
\text { dyspnoea }\end{array}$ \\
\hline
\end{tabular}

The "intention-to-treat" population contained 53 patients, while the "efficacy analysable population" - all patients who completed the study - consisted of 45 patients.

Neither in the efficacy analysis nor in the intention-io-treat analysis was any carryover effect seen between the two crossover periods. Apart from a period effect $(p<0.04)$ in supine diastolic blood pressure in the efficacy analysis - which was not present in the intention-to-treat analysis - no period effects were seen between the two crassover periods. No qualitative differences in treatment effects were present between the efficacy and intention-to-treat analysis. This means that a statistically significant difference in treatment effect in the efficacy analysis was also statistically significant in the intention-to-treat analysis and vice versa. 
Figure 3.7 shows the data on blood pressure. Standing blood pressure decreased similarly from $161 \pm 2 / 104 \pm 1 \mathrm{mmHg}$ to $143 \pm 3 / 91 \pm 2 \mathrm{mmHg}$ during enalapril and $145 \pm 3 / 89 \pm 1 \mathrm{mmHg}$ during bisoprolol. Supine blood pressure decreased from $163 \pm 2 / 102 \pm 1 \mathrm{mmHg}$ to $148 \pm 3 / 90 \pm 1$ and $144 \pm 3 / 86 \pm 1 \mathrm{mmHg}$ during enalapril and bisoprolol, respectively. Supine diastolic blood pressure was lower $(p<0.02)$ during bisoprolol than during enalapril.

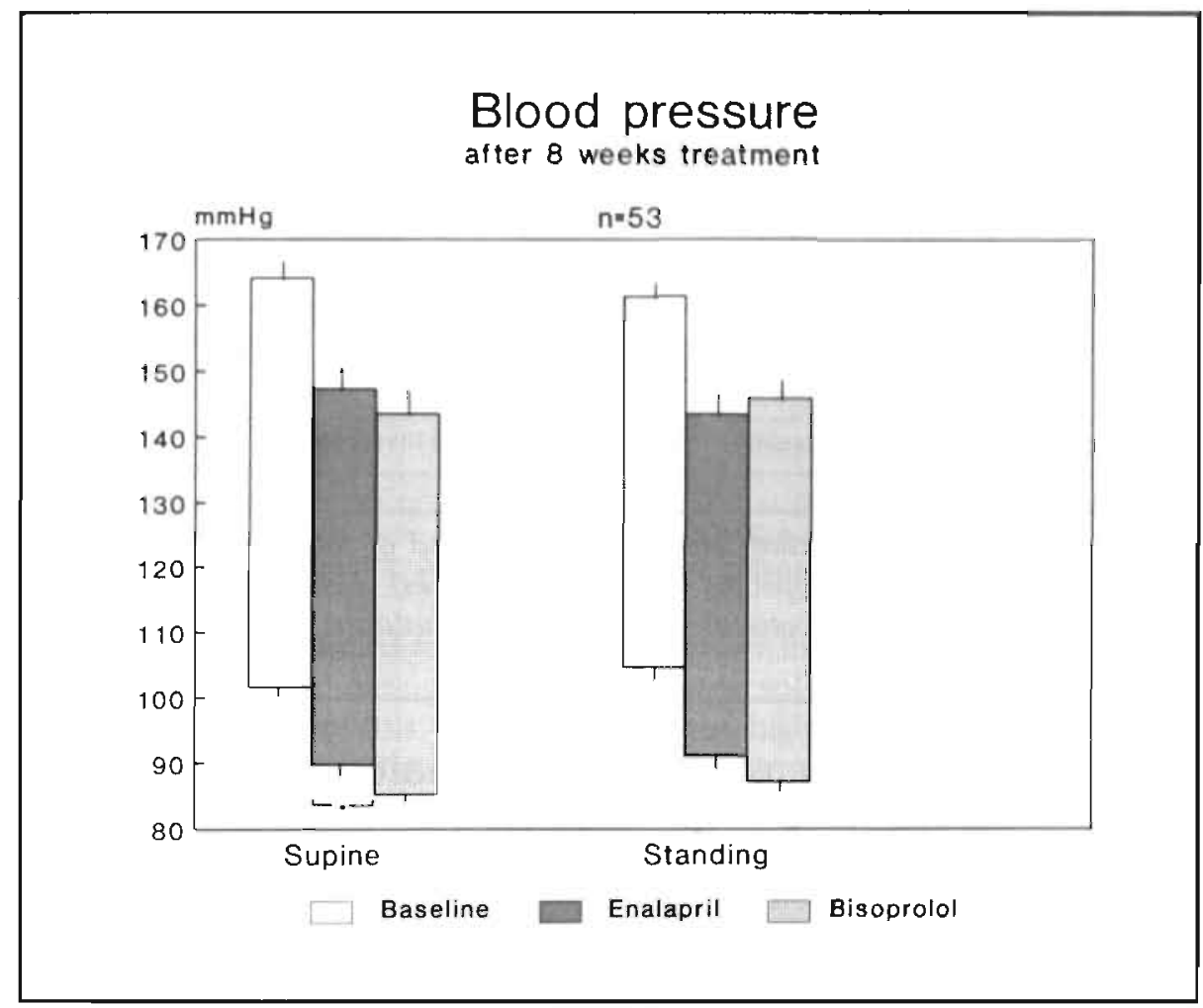

Fig 3.7. Supine and standing systolic and diastolic blood pressure at the end of the run-in period and during enalapril and bisoprolol treatment ( 8 weeks). Data are mean $\pm S E M .{ }^{*} p<0.02$, difference between bisoprolol and enalapril treatment.

Heart rate (Figure 3.8) was $84 \pm 2 \mathrm{~b} / \mathrm{min}$ in upright position and $78 \pm 2 \mathrm{~b} / \mathrm{min}$ in supine position. During bisoprolol, heart rates were lower $(p<0.001)$ in both standing $(66 \pm 2 \mathrm{~b} / \mathrm{min})$ and supine position $(63 \pm 1 \mathrm{~b} / \mathrm{min})$ compared to those during enalapril $(79 \pm 2,73 \pm 2 \mathrm{~b} / \mathrm{min}$, respectively).

Figure 3.9 shows scores of well-being as measured with the Inventory of Subjective Health. Scores were $15.8 \pm 1.0$ at baseline and did not differ between enalapril $(14.7 \pm 1.1)$ and bisoprolol $(14.0 \pm 1.1)$. The $95 \%$ confidence intervals of the differences in well-being scores between bisoprolol and enalapril ranged from -0.65 to 2.15. The retrospectively calculated power to detect a difference in quality of life score between the 2 drugs was $17 \%$. 


\section{HEART RATE}

after 8 weeks treatment

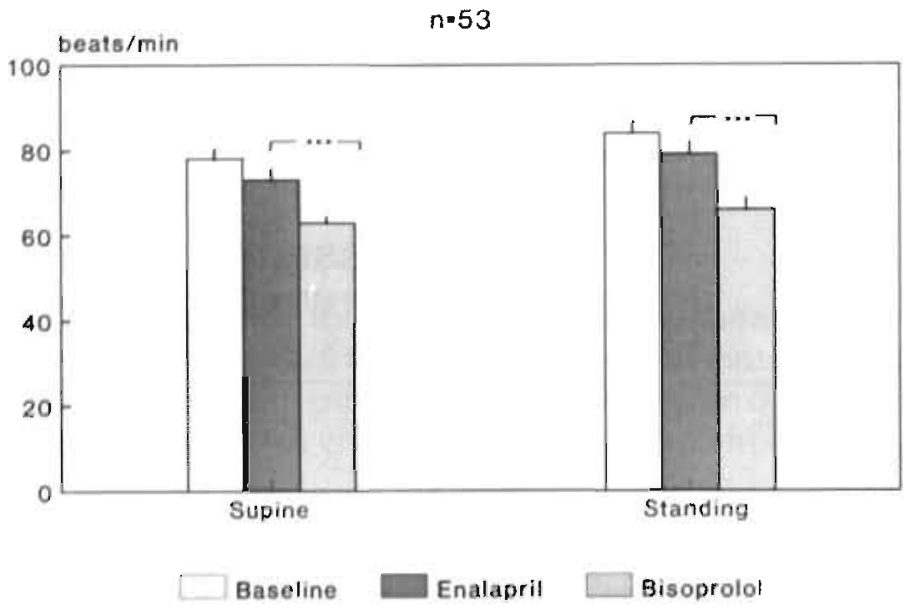

Fig. 3.8. Supine and standing beart rate at the end of the run-in period and during enalapril and bisoprolol treatment ( 8 weeks). Data are mean $\pm S E M$. *** $p<0.001$, difference between bisoprolol and enalapril treatment.

\section{Inventory of subjective health scores after 8 weeks treatment}

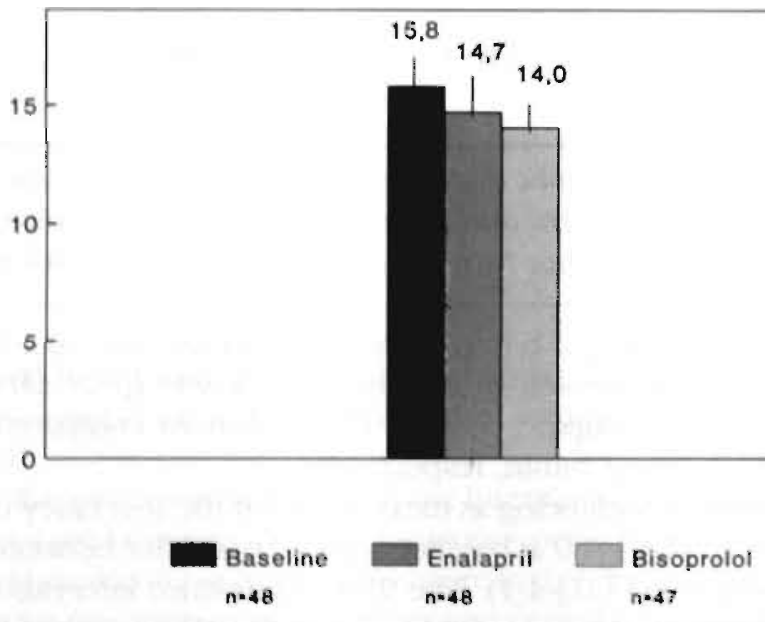

Fig. 3.9. Subjective well-being scores as measurea by the inventory of subjective bealth at the end of the run-in period and during enalapril and bisoprolol treatment (S weeks). Data are mean \pm SEM; the lower tbe score, the bigber the subjective well-being. 


\begin{tabular}{|l|c|c|c|}
\hline & run-in & enalapril & bisoprolot \\
\hline fatigue & 3 & 10 & $6(7)$ \\
\hline headache & 4 & $7(9)$ & 1 \\
\hline dizriness & 0 & $2(4)$ & $4(6)$ \\
\hline gastrocnteric & 0 & $2(3)$ & 2 \\
\hline throat complaints & 0 & $3(4)$ & 1 \\
\hline cough & 0 & $3(4)$ & 0 \\
\hline nervousness & 1 & 2 & 1 \\
\hline palpitations & 0 & 1 & $0(2)$ \\
\hline dyspnoca & $0(1)$ & $1(2)$ & $1(2)$ \\
\hline sexual disturbances & 0 & $1(2)$ & 0 \\
\hline other complaints & 1 & $8(10)$ & 7 \\
\hline TOTAL & $9(10)$ & $40(51)$ & $23(29)$ \\
\hline
\end{tabular}

Figures represent the number of patients with a complaint in the efficacy analysable population $(n=45)$. Figures in brackets show the number of patients with a complain in the intention-to-treat population $(n=53)$ if different from the efficacy analysable population.

Spontaneously mentioned adverse effects are shown in Table 3.7. Total number of complaints increased during enalapril and bisoprolol versus baseline. However, the number of complaints during enalapril was higher than during bisoprolol. During enalapril, headache and cough were regularly mentioned. Complaints of fatigue were also more frequent during enalapril than during bisoprolol. Slightly more dizziness was reported during bisoprolol treatment.

Fourty-five patients completed the study. At completion 31 patients preferred bisoprolol treatment and 11 preferred enalapril treatment. Three patients gave no preference for either drug (Figure 3.10).

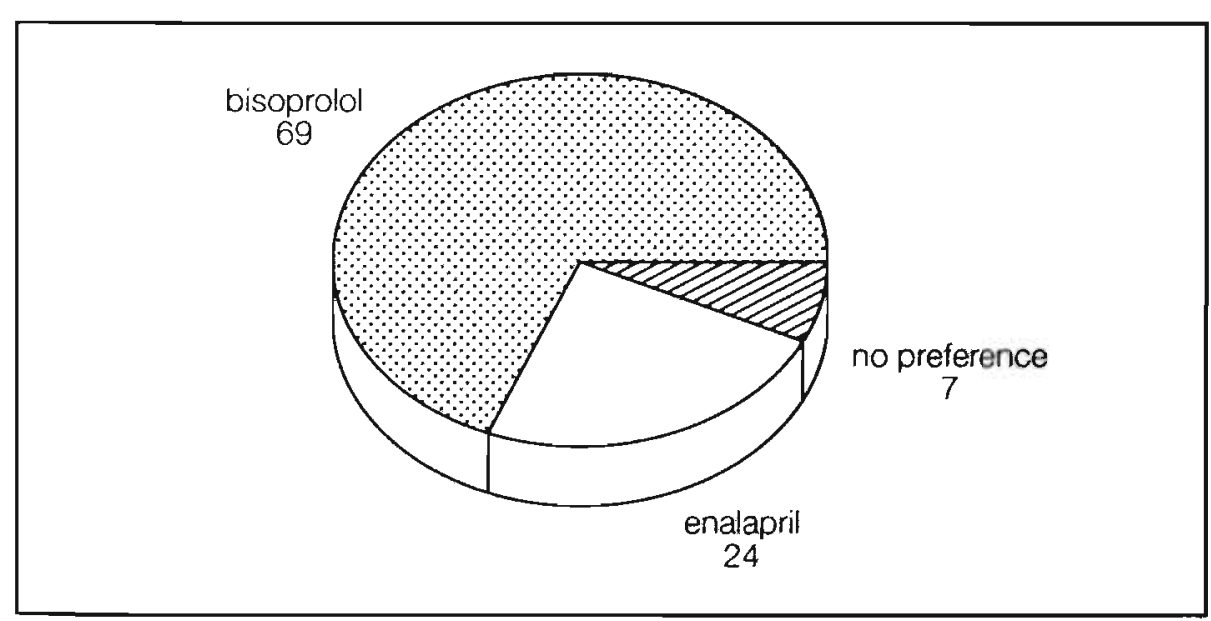

Fig 3.10. Percentage of patients' preferred drug at end of study (total $n=45$ ). 
At the end of the run-in period serum creatinine and random glucose levels averaged $91 \pm 4 \mu \mathrm{mol} / \mathrm{l}$ and $5.5 \pm 0.1 \mathrm{mmol} / \mathrm{r}$ respectively. Serum sodium was $1+1 \pm 0.3$ $\mathrm{mmol} / \mathrm{l}$ and serum potassium $4.2 \pm 0.1 \mathrm{mmol} / \mathrm{l}$. In all 4 parameters, no clinically relevant changes were found during the observation period. Body weight was $82 \pm 2 \mathrm{~kg}$ at baseline and did not change throughout the study.

Apart from a lower heart rate during bisoprolol, no clinically relevant changes in ECG were noted between the two treatment periods.

\section{discussion}

The decrease in blood pressure was at least as great with bisoprolol as with enalapril. Supine diastolic blood pressure was even lower during bisoprotol than during conalapril. A partial contribution of a slight period effect - present in the efficacy but not in the intention-to-treat analysis - in this difference cannot be excluded. As expected for a beta-adienoreceptor antagonist, bisoprolol decreased hear rate significantly compared to enalapril.

Eight paticnts dropped out because of side effects. Only 1 patient dropped out during bisoprolol treatment. Paticnt 8 (Table 3.6) dropped out carly in the second treatment period (enalapril) because of different complaints. One of them was palpitations, which might also be a bisoprolol withdrawal effect. Patient 9 dropped out during enalapril, but had already complaints of dyspnoea during bisoprolol treatment and even during the run-in period.

The adverse effects of ACE-inhibitors, such as cough, throat complaints and headache - were more often present during enalapril treatment. Fatigue is a wellknown side effect of elassical beta-blockers. It is remarkable that fatigue was more Irequenty reported during enalapril than during the highly selective beta-blocker bisoprolol. Dyspnoca and sexual disturbances were less frequenty present during bisoprolol. The total number of side effects was much higher during treatment with either agent than at baseline. This is in contrast to the results of the quality of life score. A possible explanation could be that at the end of the run-in period the physician did not interpret a complaint as an adverse effect. The total number of spontaneously mentioned complaints was lower during bisoprolol than during enalapril. It is not clear whether this is due to fewer or less severe side effects or due to a better subjective well-being perception.

In a study with a crossover design, difference in overall quality of life scores between ketanserin and placebo was shown with only 17 patients (337). Therefore, the crossover approach has been suggested useful with agents that have no carry-over effect (338). The present comparative study uses the crossover design with a sample size of 54 patients, which is 3 times that of the abovementioned placebo-controlled study. No carry-over effect was present as measured with the Koch procedure for the two-way crossover design.

The quality of life score, as measured with the Inventory of Subjective Health, was better during treatment with bisoprolol and enalapril than at the end of the run-in period. The results for enalapril are in accordance with other studies, where global scores of quality of life were improved with enalapril versus baseline $(313,341)$. This improvement versus baseline might - at least in part - be due to the participation in the trial (339). A real improvement in quality of life can only be proven in a placebo-controlled study. In the present study, no statistically significant difference in quality of life scores was observed between the two drugs. This is in 
accordance with results of other recent studies, comparing quality of life during treatment with the selective $B_{1}$-adrenoceptor antagonist atenolof versus an ACEinhibitor (341,344). However, in the present study, the score of the Inventory of Subjective Health even tended to be somewhat better during bisoprolol treatment than during enalapril. A larger sample size might provide a statistically significant difference. Since - with the sample size used- retrospectively, the power of the test to detect a difference in score between the 2 drugs is not more than $17 \%$, the sample size needed for $80 \%$ power has to be substantially larger. Moreover, a small but statistically significant difference does not imply clinical relevance. The fact that $31(69 \%)$ of the 45 patients, who made a blinded treatment choice at completion of the study, have selected the bisoprolol treatment period, and that less adverse effects were reported during bisoprolol, suggests that the slightly (but not statistically significant) better quality of life score during bisoprolol might be of clinical relevance.

In conclusion : in patients with mild-to-moderate hypertension, bisoprolol decreased blood pressure at least as well as enalapril. At the dosages used, bisoprolol had less side effects than enalapril. The well-being score during bisoprolol treatment tended to be better than during enalapril and more patients mentioned to feel better during bisoprolol. This study does not confirm the assumption that ACEinhibitors may cause fewer side effects than beta-blocking agents, and certainly not than selective $B_{1}$-blockers. 


\section{chapter 3. Exercise tolerance during antihypertensive treatment $^{3}$}

\section{introduction}

Beta-blocking agents are widely used in the treatment of hypertension and cardiac diseases. Patients treated with these drugs often complain of fatigue during exercise. Maximal exercise capacity $(345-349)$ and endurance performance $(314-320)$ are diminished during beta-adrenoceptor blockade in hypertensive as well as in normotensive subjects. Drug-induced changes in haemodynamics predominantly influence maximal exercise capacity, altough metabolic effects play an important role during endurance performance (350). A decreased exercise performance can alter the quality of life of physically active patients. On the other hand, betablocking agents can increase exercise capacity in patients with angina pectoris by delaying pain and other ischaemic signs. These anti-ischaemic effects of betablocking drugs are outside the scope of this study.

Nebivolol is a newly developed selective beta-adrenoceptor antagonist with a particular haemodynamic profile. This profile has been described in chapter 1. In hypertensive patients, the antihypertensive effect of nebivolol $5 \mathrm{mg}$ once daily was greater than that of $2.5 \mathrm{mg}$ once daily. Ten $\mathrm{mg} /$ day did not enhance the antihypertensive effect (303-304).

In this study, the effects of nebivolol on maximal and endurance exercise performance were compared with those of atenolol. The haemodynamic and metabolic effects of the two drugs during exercise were also studied. Since atenolol and nebivolol are antihypertensive drugs, comparison of dosages, inducing a similar blood pressure lowering effect at rest, is clinically relevant.

In a pilot study, atenolol $100 \mathrm{mg}$ and nebivolol $5 \mathrm{mg}$ once daily decreased blood pressure equally in healthy subjects. The equipotent antihypertensive effect of atenolol $100 \mathrm{mg}$ and nebivolol $5 \mathrm{mg}$ once daily has also been shown in hypertensive patients by means of 24-hour ambulatory blood pressure monitoring (351).

\section{subjects and methods}

\section{Subjects}

Before the study, voluntecrs underwent a progressive maximal exercise test for determination of maximal aerobic work capacity $\left(\mathrm{W}_{\text {max }}\right)$ on a bicycle ergometer. Females started the exercise test at $50 \mathrm{~W}$, while males started at $100 \mathrm{~W}$ workload foi 5 minutes. Subsequently, workload was increased every 3 minutes by $50 \mathrm{~W}$ for $25 \mathrm{~W}$ if heart rate was more than 160 beats/min), until exhaustion. Volunteers who were able to perform an endurance exercise test (at $70 \%$ of the predetermined $\mathrm{W}_{\max }$ ) for at least 30 minutes were selected.

Twenty-one apparently healthy volunteers entered and completed the study. Volunteers (males 15 ; females 0 ) were $23 \pm 1$ yr (19-31 yr); their weight was $71 \pm 2 \mathrm{~kg}$ $(53-86 \mathrm{~kg})$ and their height was $178 \pm 2 \mathrm{~cm}(160-192 \mathrm{~cm})$. In all volunteers serum levels of liver enzymes were less than 3 times the upper limit of normal and serum creatinine was $<115 \mu \mathrm{mol} / \mathrm{l}$. Volumteers were active in sports at least on a recreational level. Their maximal aerobic exercise capacity ranged from 160 to $367 \mathrm{~W}$.

\footnotetext{
3 based on: LMAB Van Bortel, MA van Baak. Exercise tolerance with nebivolol and atenolol Cardiovase Drugs Ther 1992, 6:339-247.
} 


\section{Study design}

Volunteers entered a double blind placebo-controlled three-way crossover study and were given in random order nebivolol (solution) $5 \mathrm{mg}$, atenolol (tablets) $100 \mathrm{mg}$, and placebo once daily for 2 weeks each, following the double dummy technique. Between treatment periods, there was a 3-week drug-free wash-out period. Atenolol and placebo tablets as well as nebivolol and placebo solution were identical in appearance. On day 10 of each treatment period, voluntecrs performed a progressive maximal exercise test. On day 14 a submaximal endurance test until exhaustion (at $70 \%$ of predetermined $W_{\text {max }}$ ) was performed on a bicycle ergometer (Lode, Groningen, The Netherlands). Exhaustion was defined as the moment at which pedalling rate could not be maintained above $50 \mathrm{rpm}$. Exercise tests were performed when peak effects of atenolol and nebivolol were anticipated, 3-4 hours after the last administration of the drug. Exercise tests in each subject were also carried out on the same time of the day. Volunteers were asked to keep all factors - and especially physical exercise - as constant as possible throughout the whole study period. In addition, subjects were asked not to consume alcohol, coffee, or nicotine on the day of the test and to avoid strenuous physical exercise during 24 hours preceding the test. Volunteers were also asked to take a small meal 2 hours before the test. The study was approved by the Ethical Committee of the University of Limburg. All volunteers gave their written informed consent. Volunteers were asked about their compliance. Volunteers compliance was also checked by counting tablets and by measuring plasma levels of the drugs.

\section{Protocol of the maximal exercise test}

After cannulation of an antecubital vein blood was drawn for bascline analysis. Resting blood pressure and heart rate were measured in sitting position after 10 minutes of rest. Exercise started at $100 \mathrm{~W}$ or $50 \%$ of the predetermined maximal work capacity $\left(W_{\max }\right)$ if $<100 \mathrm{~W}$ for 6 minutes. Subsequently, workload was increased with $10 \%$ of predetermined $W_{\max }$ every 3 minutes until exhaustion. At $100 \mathrm{~W}$ or $50 \%$ of predetermined $W_{\text {max }}$ blood pressure was measured and cardiac output was assessed using the $\mathrm{CO}_{2}$ rebreathing technique (352-353). Variation of this technique is less than $5 \%$ during exercise $(354-355)$.

Throughout the test minute ventilation $\left(\mathrm{V}_{\mathrm{E}}\right), \mathrm{O}_{2}$ consumption $\left(\mathrm{VO}_{2}\right)$ and respiratory exchange ratio (R) were measured continuously and averaged per $30 \mathrm{~s}$. During the last $30 \mathrm{~s}$ of each work load, at exhaustion and after 3 min recovery, systolic blood pressure (up to $80 \% \mathrm{~W}_{\max }$ ), heart rate and perceived exertion were measured and venous blood was drawn for determination of lactate, glucose, potassium, glycerol and non-esterified fatty acid (NEFA) concentrations.

\section{Protocol of the submaximal endurance test}

After cannulation of an antecubital vein blood was drawn for baseline analysis. Resting blood pressure and heart rate were measured in sitting position after 10 minutes of rest. Submaximal exercise at $70 \%$ of the predetermined maximal work capacity was performed until exhaustion or for $90 \mathrm{~min}$. At regular time intervals $(5,10,15,20,30,40,50,60,70,80$ and $90 \mathrm{~min})$ during exercise, at exhaustion, and at min 3, 6 and 10 during recovery, systolic blood pressure, heart rate and perceived exertion were measured and blood was drawn for determination of 
haemoglobin, haematocrit, potassium, glucose, glycerol, NEFA and lactate. From changes in haemoglobin and haematocrit, changes in plasma volume during exercise were calculated according to Costill and Fink (356).

\section{Methods}

Heart rate (HR) was measured by ECG and blood pressure with a mercury sphygmomanometer (resting diastolic blood pressure: Korotkoff V; diastolic blood pressure at $100 \mathrm{~W}$ or $50 \% \mathrm{~W}_{\max }$ exercise: Korotkoff IV). Minute ventilation ( $\mathrm{V}_{\mathrm{E}}$ ), oxygen consumption $\left(\mathrm{VO}_{2}\right)$, respiratory exchange ratio (R) and cardiac output $\left(\mathrm{Q} ; \mathrm{CO}_{2}\right.$ rebreathing) were measured with an automated device (Eos sprint, Jäger, Freiburg, Germany). Perceived exertion was measured using the Borg scale (357). Blood was immediately centrifuged for $1 \mathrm{~min}$. Serum and plasma were rapidly frozen in dry ice and stored at $-70^{\circ} \mathrm{C}$ until assayed. Plasma levels of glucose and lactate (Cobas auto-analyzer) and serum levels of glycerol (a modification of the triglyceride (Neutralfett) test combination Boehringer Mannheim) were measured enzymatically. Serum NEFA concentrations were determined colorimetrically (358). Plasma levels of $\mathrm{K}+$ were determined by flame photometry (Instrumentation laboratory 243). Plasma levels of atcnolol and nebivolol were determined at bascline ( 3 hours after the last administration of the drug) and assayed by HPLC (359-360).

\section{Data analysis}

Data are presented as mean \pm S.E.M. Data in figures are shown by bars and curves. $\mathrm{X}$-axis of maximal exercise curve figures show data at rest, at $100 \mathrm{~W}$ and at increasing workloads, expressed as percentage of the predetermined $W_{\text {max }} X$ axis of cndurance exercise curve figures is a time axis, representing the number of ninutes cycled by the subjects. Bars and curves show means of all 21 volunteers. This means that in each crossover period all volunteers cycled up to $80 \%$ or more of their predetermined $\mathrm{W}_{\max }$ during the maximal exercise test and for at least 20 minutes during the endurance test. The last point during exercise is the value at exhaustion, which is shown at the appropriate average workload or exercise time. Recovery starts at exhaustion. Therefore, values representing exhaustion and 0 minutes of recovery are identical.

Statistical analysis was performed by non-parametric analysis of variance and Zerbe's randomization test for curves (36i) with Scheffe's procedure for intergroup comparison. $\mathrm{P}<0.05$ was considered statistically significant. The following calculations were made: stroke volume $(\mathrm{SV})=\mathrm{Q} / \mathrm{HR}$; mean arterial pressure (MAP) $=$ diastolic blood pressure $(\mathrm{DBP})+1 / 3$ (systolic blood pressure $(\mathrm{SBP})-\mathrm{DBP})$; total peripheral resistance $(\mathrm{TPR})=\mathrm{MAP} / \mathrm{Q}$.

\section{results}

No major changes in volunteers' physical activity were reponed during the study. Volunteer compliance was good as measured by counting tablets (never less than 13 tablets were taken during the 14 -day period), measuring solution volume and by deiection of atenolol and nebivolol in plasma samples. All volunteers had detectable plasma levels of atenolol and nebivolol in the appropriate period. Plasma levels of atenolol ranged from 129 to $959 \mathrm{ng} / \mathrm{ml}$. Apart from 3 subjects with higher nebivolol plasma levels $(8.5,14.6$, and $17.8 \mathrm{ng} / \mathrm{ml}$, respectively), plasma 
levels of nebivolol ranged from 0.17 to $3.07 \mathrm{ng} / \mathrm{ml}$. Data on resting blood pressure and heart rate are shown in Fig 3.11. Mean arterial pressure (MAP) at rest was $91 \pm 1 \mathrm{mmHg}$ (SBP: $118 \pm 3 \mathrm{mmHg}$ : DBP : $77 \pm 1 \mathrm{mmHg}$ ) and decreased $(\mathrm{p}<0.01)$ similarly during atenolol $(83 \pm 2 \mathrm{mmHg})$ and nebivolol $(84 \pm 2 \mathrm{mmHg})$. Heart rate was $82 \pm 4 \mathrm{~b} / \mathrm{min}$ during placebo and decreased $(\mathrm{p}<0.001)$ during atenolol $(62 \pm 2 \mathrm{~b} / \mathrm{min})$ and nebivolol $(68 \pm 3 \mathrm{~b} / \mathrm{min})$.

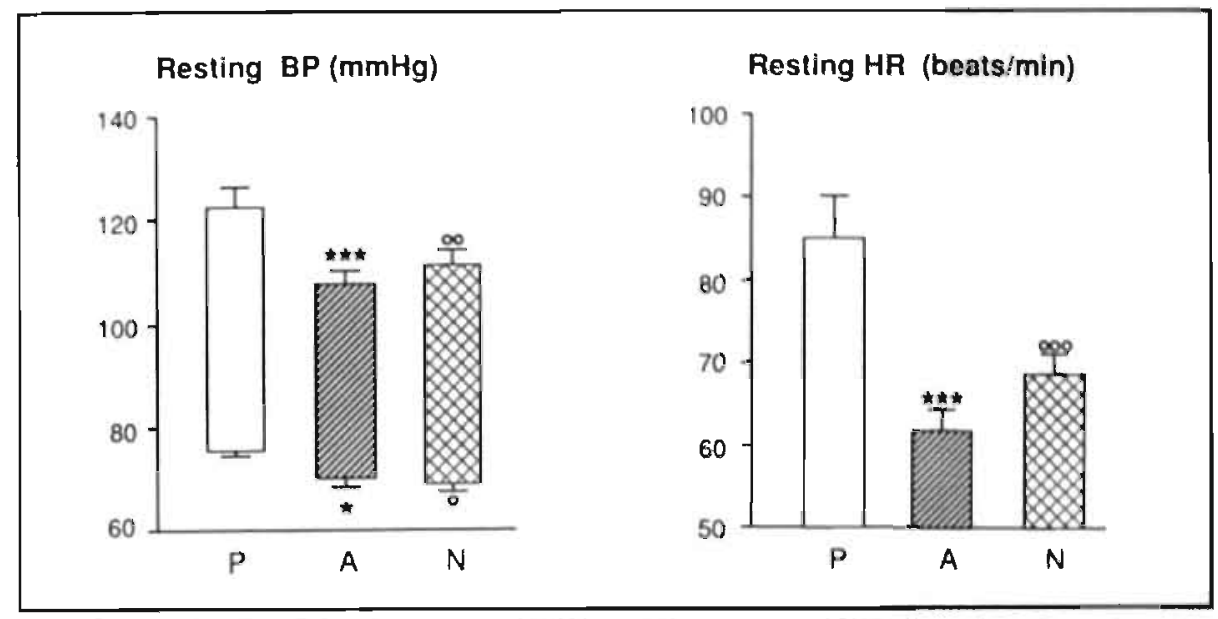

Fig. 3.11. Resting blood pressure (BP) and beart rate (HR) for placebo, atenolol and nebivolol. $\square$ placebo; atenolol; nebivolol; $" p<0.05$. ${ }^{*} p<0.001$ (atenolol-placebo); ${ }^{\circ} p<0.05,{ }^{\circ} p<0.01,{ }^{\circ 0}{ }^{\circ} p<0.001$ (nebivolol-placebo).

Haemodynamics during exercise at $100 \mathrm{~W}$ are shown in Table 3.8. MAP and heart rate decreased $(p<0.005)$ during atenolol and nebivolol at $100 \mathrm{~W}$ exercise. The atenolol induced heart rate reduction was larger $(p<0.005)$ than that due to nebivolol. At $100 \mathrm{~W}$ exercise, stroke volume increased $(p<0.001)$ similarly during atenolol and nebivolol. In contrast to atenolol, nebivolol tended (ns) to increase cardiac output. Nebivolol decreased $(p<0.03)$ calculated total peripheral resistance during exercise (100 W).

Table 3.8. Haemodynamics during exercise at $100 \mathrm{~W}$ (mean \pm S.E.M.)

\begin{tabular}{|l|c|c|c|}
\hline & Placebo & Atenolol & Nebivolol \\
\hline Heart rate (b.min $\left.{ }^{-1}\right)$ & $121 \pm 4$ & $94 \pm 3^{\circ}$ & $102 \pm 3^{\circ \circ}$ \\
\hline Systolic BP' (mmHg) & $153 \pm 4$ & $134 \pm 3^{\circ}$ & $1.38 \pm 3^{\circ}$ \\
\hline Diastolic BP (mmHg) & $75 \pm 3$ & $71 \pm 2$ & $70 \pm 2$ \\
\hline Mean arterial pressure (mmHg) & $101 \pm 3$ & $92 \pm 2^{\circ}$ & $92 \pm 2^{\circ}$ \\
\hline Cardiac output $\left(\mathrm{mmin} \mathrm{m}^{-1}\right)$ & $14.3 \pm 0.6$ & $14.2 \pm 0.8$ & $15.0 \pm 0.8$ \\
\hline Stroke volume $(\mathrm{ml})$ & $121 \pm 6$ & $156 \pm 11^{\circ}$ & $152 \pm 11^{\circ}$ \\
\hline Total peripheral resistance (mmHg.min. $\left.\mathrm{I}^{-1}\right)$ & $7.2 \pm 0.2$ & $6.8 \pm 0.3$ & $6.5 \pm 0.3^{\circ}$ \\
\hline
\end{tabular}

- Significant difference between placebo and atenolol: ${ }^{\circ}$ Significant difference berween placebo and nebivolol; ${ }^{\circ}$ Significant difference between atenolol and nebivolol 
Systolic blood pressure and heart rate curves during exercise are shown in Fig 3.12. Rise in systolic blood pressure was significantly smaller with atenolol than with nebivolol during endurance performance and tended to be smaller during maximal exercise. Exercise-induced tachycardia was less depressed with nebivolol than with atenolol during maximal and endurance exercise. Maximal heart rate averaged $187 \mathrm{~b} / \mathrm{min}$ during placebo and decreased to $161 \mathrm{~b} / \mathrm{min}$ with nebivolol (14\%) and $140 \mathrm{~b} / \mathrm{min}$ with atenolol $(25 \%)$.

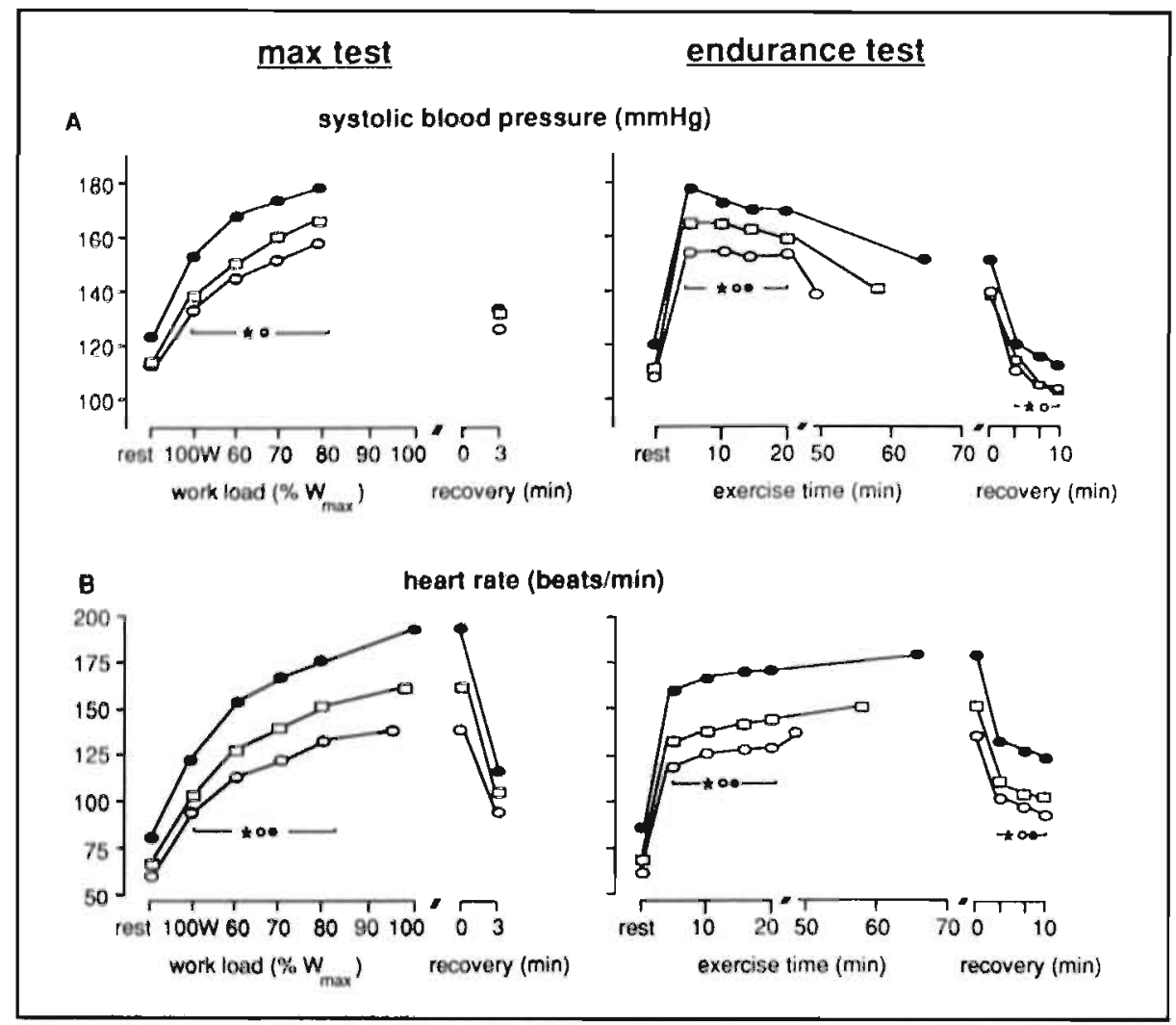

Fig. 3.12. Systolic blood pressure and beart rate curves during maximal and endurance exercise tests. placebo; $O$ atenolol ; $\square$ nebivolol. Significan difference between: "atenolol-placebo, "nebivolol-placebo, "atenolot-nebivolol. Fig. A: SEM did not exceed $6 \mathrm{mmHg}$ for placebo and nebivolol and $5 \mathrm{mmHg}$ for atemolol. Fig. B: SEM did not exceed $5 \mathrm{beats} / \mathrm{min}$ for placebo and $3 \mathrm{beat} / \mathrm{min}$ for atemolol and nebinolol.

Maximal work load was $263 \pm 15 \mathrm{~W}$ during placebo, $251 \pm 15 \mathrm{~W}$ during atenolol $\left(\mathrm{p}<0.02\right.$ ) and $256 \pm 16 \mathrm{~W}$ during nebivolol (n.s.). $\mathrm{VO}_{2 \max }$ was $3.40 \pm 0.17 \mathrm{l} / \mathrm{min}$ during placebo and was decreased $(p<0.03)$ during atenolol $(3.22 \pm 0.19 \mathrm{l} / \mathrm{min})$ and was $3.37 \pm 0.19 \mathrm{l} / \mathrm{min}$ (n.s.) during nebivolol (Fig 3.13). Submaximal endurance time was reduced from $65 \pm 5 \mathrm{~min}$ during placebo to $50 \pm 5 \mathrm{~min}$ during atenolol $(p<0.001)$ and was not significantly affected during nebivolol $(61 \pm 5 \mathrm{~min})$ (Fig 3.13). 


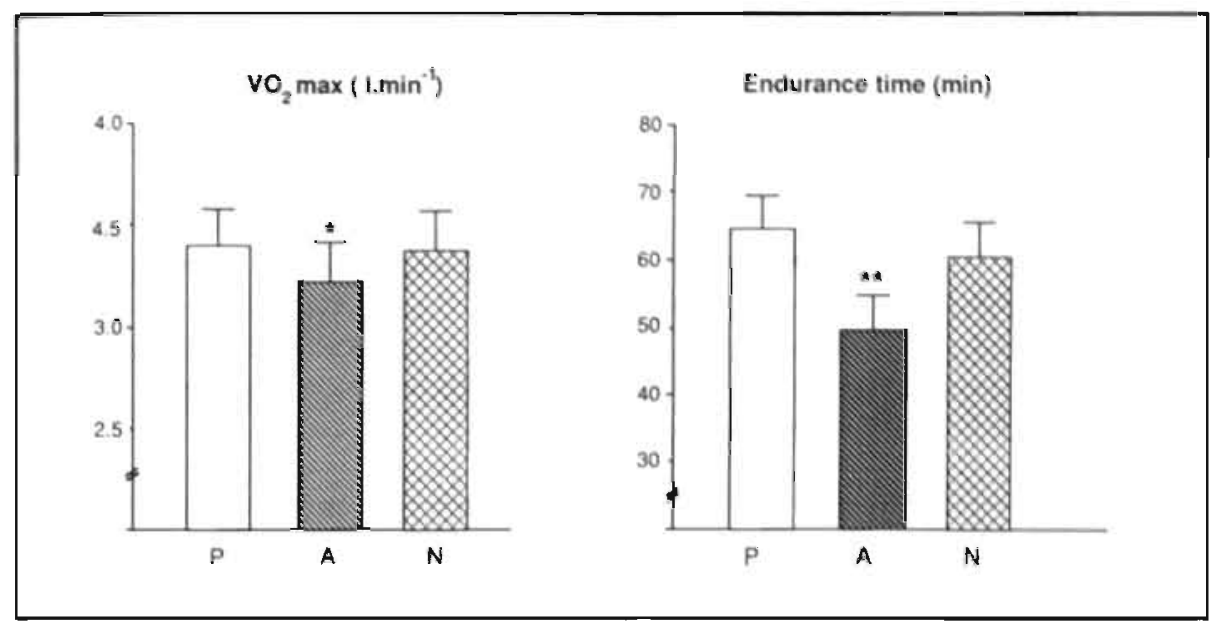

Fig. 3. 13. Maximal oxygen consumption $\left(\mathrm{VO}_{2} \max\right.$ ) and endurance exercise time during placebo, atenolol and nebivolol. $\square$ placebo; atenolol ; nebiwolol " $p<0.05, * p<0.01$ (atenolol-placebo).

During maximal exercise testing, no significant differences in perceived exertion rating (RPE) (Fig 3.14) curves were seen between placebo, atenolol and nebivolol. During endurance exercise RPE curves did not differ between placebo and nebivolol, but RPE was significantly $(p<0.02)$ increased with atenolol.

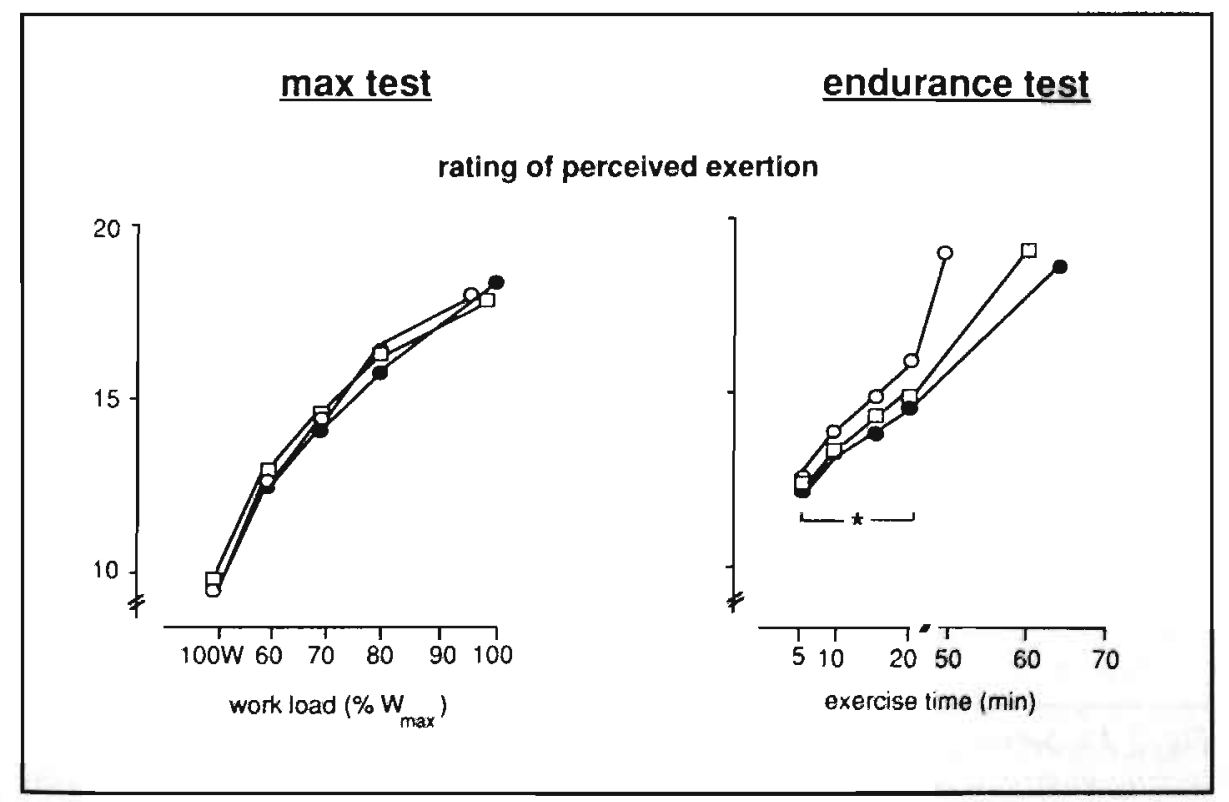

Fig. 3.14. Rating of perceived exertion measured with the 20-point Borg scale during maximal and endurance exercise tests. placebo; $O$ atenolol; $\square$ nebivolol. Significant difference between: *atenolol-placebo. SEM did not exceed 0.5 for placebo, atenolol and nebivolol. 
Serum NEFA and glycerol concentration curves are shown in Fig 3.15. During recovery of endurance exercise, NEFA and glycerol concentrations were reduced during atenolol and nebivolol as compared to placebo. These reductions tended to be more pronounced during atenolol than during nebivolol. Plasma potassium concentrations (Fig 3.16) during endurance exercise were significandy higher during atenolol than during placebo and nebivolol $(p<0.02)$. Although lactate concentration curves did not differ during exercise, lactate concentrations at maximal exercise were lower during atenolol $(10.0 \pm 0.6 \mathrm{mmol} / \mathrm{l} ; \mathrm{p}<0.05)$ and

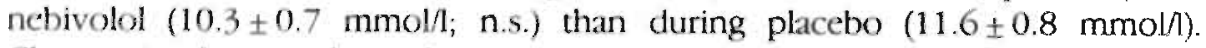
Changes in plasma volume during exercise and plasma concentrations of sodium and glucose did not differ between the 3 groups.

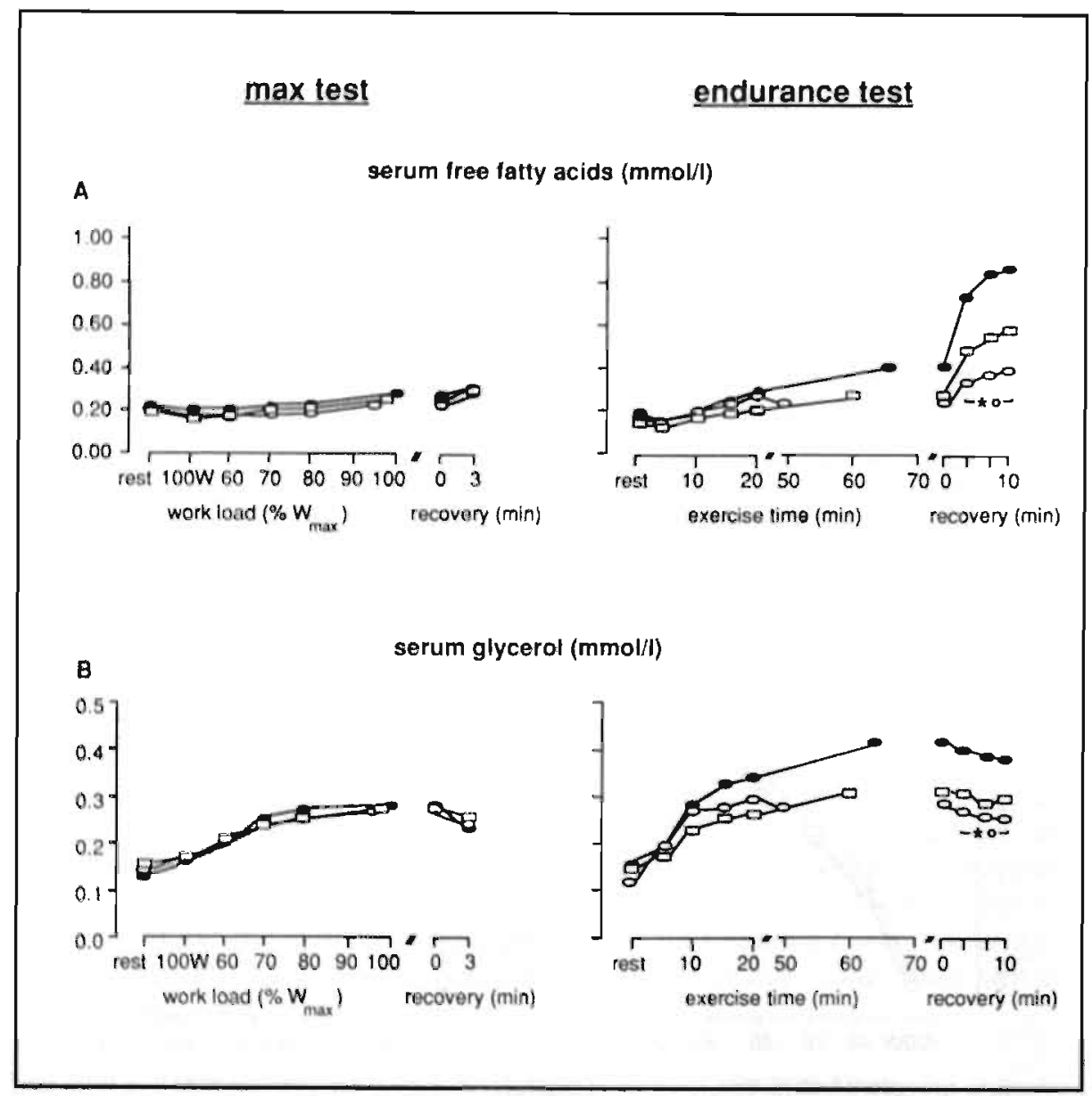

Fig. 3.15. Serum free fatty acid (A) and serum glycerol (B) concentration curves during maximal and endurance exercise tests. placebo; $\bigcirc$ atenolol; $\square$ nebivolol. Significant difference between: "atenolol-placebo, ' nebivolol-placebo. Fig. A: SEM did not exceed $0.08,0.03$ and $0.07 \mathrm{mmol} / \mathrm{for}$ placebo, atenolol and nebivolol respectively. Fig. B: SEM did not exceed 0.03, 0.04 and $0.02 \mathrm{mmol} / \mathrm{for}$ placebo, atenolol and nebivolol respectively. 


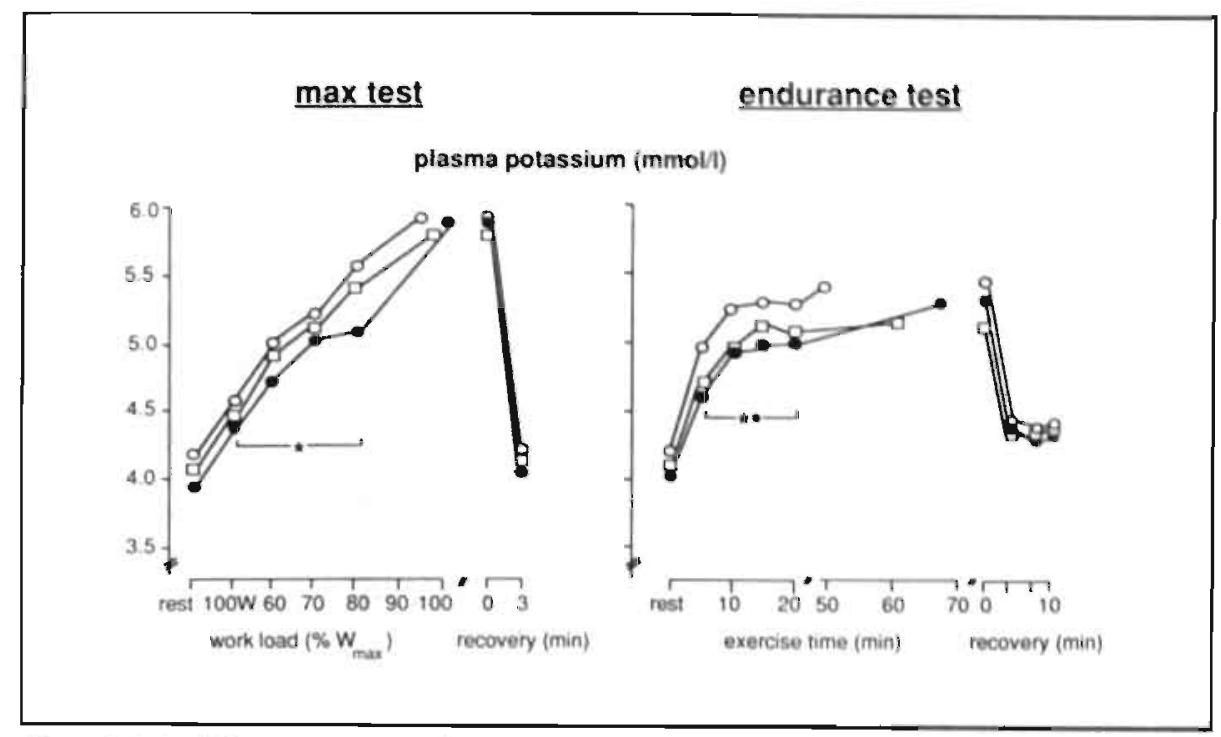

Fig. 3.16. Plasma potassium concentration cunves during maximal and endurance exercise tests. placebo; $\bigcirc$ atenolol; $\square$ nebivolol Significam difference between: "atenolol-placebo, "atenolol-nebivolol. SEM did not exceed $0.1 \mathrm{mmol}$ for placebo, atenolol and nebivolol.

\section{discussion}

As stated in the introduction, the aim of this study was to investigate the reduction in exercise capacity, a well-known and - for physically active patients - disturbing side-effect of classical beta-blocking drugs. This study did not investigate the - for patients with angina pectoris - beneficial effect of beta-blocking drugs on exercise time by delaying pain and other symptoms of heart ischaemia.

Volunteers' heart rate at rest was relatively high during placebo. This might at least in part be explained, on the one hand, by the position of the volunteer and timing of the measurement - immediately before starting the exercise test with the volunteer already sitting on the bicycle ergometer - and, on the other hand, by the volunteer study population - all volunteers were familiar with physical exercise, but the majority were not well-trained athletes. Based on plasma levels of nebivolol, 2 volunteers are probably poor metabolizers for nebivolol while 1 might be an intermediate metabolizer. This is in accordance with the incidence of poor metabolizers for debrisoquine in the general population (362).

This study shows that in apparently healthy subjects nebivolol $5 \mathrm{mg}$ once daily and atenolol $100 \mathrm{mg}$ once daily cause an equivalent decrease in resting blood pressure. In contrast, atenolol tended to reduce heart rate at rest more than nebivolol.

Maximal exercise capacity is reduced during beta-adrenoceptor blockade in normotensive as well as in hypertensive subjects (345-349). This reduction ranged from $2-15 \%(363)$. In confirmation of these observations, in this study maximal work capacity was statistically significantly decreased $(5 \%)$ during atenolol treatment, but was not reduced during nebivolol.

Since, during normal and recreational physical activity, maximal work capacity will usually not be reached, a small decrease in maximal work capacity does not need 
to alter the perception of the quality of life. However, reduced exercise endurance performance at submaximal exercise may alter the quality of life in physically active patients. Reduction in endurance capacity during beta-adrenoceptor blockade has been well documented (314-320). In this study atenolol decreased endurance time significantly (23\%). In contrast to classical beta-adrenoceptor blocking drugs, nebivolol did not decrease $(6 \%)$ endurance time significantly in healthy volunteers. Since in previous studies with classical beta-blockers no difference in effect on exercise capacity has been found between normotensive and hypertensive subjects (363), for nebivolol similar results might be expected in hypertensive patients. The effect of different antihypertensive drugs on exercise endurance performance is summarized in Fig. 3.17. In 8 studies (314-315, 317-319, 364-366) with non-selective R-blockers (propranolol, pindolol, nadolol), the decrease in endurance time ranged from 25 to $51 \%$ and was on average $40 \%$. During selective R-blockade (315-317,319-320,365 and present study) with atenolol, metoprolol, or epanolol, decrease in endurance time was $26 \%(14-39 \%)$. Two studies are published on the calcium-antagonist verapamil. One study mentioned a $16 \%$ reduction (364), the other (320) a reduction in endurance time of $7 \%$ (mean of the 2 studies: $11 \%$ ). Endurance time reduction was 3 and $12 \%$ with enalapril and $9 \%$ with captopril $(320,367-368)$; mean of the 3 studies with ACEinhibitors: $8 \%$. The reduction in endurance time during nebivolol was comparable with that during antihypertensive treatment with calcium-antagonists and ACE-inhibitors. Complaints related to a small decrease in endurance exercise capacity are not common during treatment with calcium-antagonists and $\mathrm{ACE}$ inhibitors. This suggests that during nebivolol, quality of life might also be pre-



Fig 3.17. Decrease in endurance time during treatment witb different antibypertensive drugs. Data are means of different shidies. NB. non-selective B-blockers; $S B$, selective $\beta_{l}$-blockers; $C A$, calcium-antagonists; $A I, A C E$ inbibitors; NE, nebivolol. 
served in this respect. This possible difference in effect on quality of life between nebivolol and classical beta-blockers is supported by the results obtained with the Borg scale. Classical beta-blockers increase perceived exertion (347). In the present study atenolol also increased perceived exertion during endurance exercise performance, while nebivolol did not significantly

In this study, both at rest and at $100 \mathrm{~W}$ exercise, mean arterial pressure decreased similarly during atenolol and nebivolol, though heart rate decreased more during. atenolol. Stroke volume was similarly increased during atenolol and nebisolof at 100 W exercise. During exercise cardiac output is usually decreased by betablocking agents (363). In contrast to our findings in a previous study (349), in the present study, no significant decrease in cardiac output was seen during atenolol at $100 \mathrm{~W}$ exercise. However, cardiac output tended to be higher during nebivolol than during atenolol. Total peripheral resistance decreased during nebivolol at $100 \mathrm{~W}$ exercise. It is not clear whether this effect was due to less beta -blockade or to some slight vasodilating properties of nebivolol which might explain why, despite less cardiac beta-blockade, in healthy subjects nebivolol $5 \mathrm{mg}$ daily shows a similar antihypertensive effect as atenolol $100 \mathrm{mg}$ daily.

During endurance exercise at $70 \%$ of the predetermined maximal work capacity (which is a work load $>100 \mathrm{~W}$ ), not only heart rate but also systolic blood pressure was lower with atenolol than with nebivolol, suggesting a larger degree of betablockade with atenolol.

The haemodynamic effects of beta-blockers are thought to play a major role in the alterations of maximal exercise capacity. A positive relationship between $\%$ reduction in maximal heart rate and in maximal work capacity has been shown with a threshold at $15-20 \%$ reduction. A maximal heart rate reduction below this threshold did not decrease maximal work capacity (369). In this study maximal heart rate reduction with nebivolol was below the threshold $(14 \%)$, while that with atcnolol was above the threshold (25\%). This might explain why ncbivolol did not change maximal exercise capacity.

During endurance performance metabolic effects play an important role (350). With regard to metabolic aspects, plasma glucose levels during beta-blockade were decreased during exercise in fasted subjects and also during maximal exercise with betaz-blockade (363). In this study, no change in plasma glucose levels was seen. Decreased glucose levels were not expected, since subjects were not fasting and

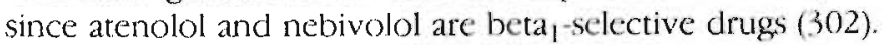

The literature data on the influence of beta-blockade on plasma lactate during max imal and during endurance exercise are inconsistent. In the present study plasma lactate concentration curves did not differ between placebo, atenolol and nebivolol.

NEFA are an important energy source for skeletal muscle, especially during prolonged exercise (363). Serum levels of NEFA and glycerol are a result of formation by lipolysis and utilization for energy supply and gluconeogenesis. During recovery from exercise, NEFA utilization decreases abruptly, while NEFA production continues for some minutes. Therefore, the increase in serum NEFA concentration during recovery is a measure of lipolysis. Lipolysis is inhibited by beta-blocking drugs. This inhibition was more pronounced during atenolol than during nebivolol. During and after maximal exercise; serum glycerol and NEFA concentrations did not differ between placebo, atenolol and nebivolol. The lack 
of increased NEFA. levels after maximal exercise suggests that during maximal exercise lipolysis is not important for energy supply.

During beta-blockade, plasma potassium increases during exercise due to a reduced uptake of $\mathrm{K}^{+}$in inactive tissues (370). The increase in $\mathrm{K}^{+}$concentration appears similar after beta, and non-selective beta-adrenoceptor blockade (317, 371-372). The loss of potassium during muscle contractions might affect muscle membrane excitability, reduce contractility and increase fatigue (373). In this study plasma potassium was increased during atenolol bur not during nebivolol.

This study shows that, at dosages of atenolol and nebivolol which produce a similar blood pressure reduction at rest and at $100 \mathrm{~W}$ exercise, nebivolol does not alter maximal work capacity nor endurance performance, nor perceived exertion. These objective (endurance time) and subjective (perceived exertion) findings are unexpected for a beta-blocking drug and suggest that during nebivolol treatment quality of life perception in physically active patients might be preserved. Two explanations for these surprising findings may be considered: a smaller degree of beta-blockade during nebivolol or an ancillary property, as also suggested by previous studies (302). The haemodynamic (heart rate and systolic blood pressure) and metabolic (glycerol and NEFA) effects, and the change in plasma potassium during exercise indicate that, at the dosages used, atenolol has at more pronounced beta-blocking effect than nebivolol. In confirmation of literature data (370), this different degree of beta-blockade might explain the difference in maximal work capacity between nebivolol and atenolol. Although not fully established, such a relationship between the degree of beta-blockade and endurance performance is very likely. This might explain why the well-documented reduction in endurance capacity during betablocker treatment does not seem present during treatment with nebivolol becaluse full beta-blockade is not achieved with nebivolol $5 \mathrm{mg}$ once daily. However, an incillary property, which might influence endurance capacity favorably cannot be excluded. Further investigation is needed to determine the equipotent beta-blocking dosages for atenolol and nebivolol and to investigate the nature of a possible ancillary antihypertensive property of nebivolol.

So far all antihypertensive drugs induced a decrease in endurance performance, albeit not always statistically significant. A small decrease up to $16 \%$ (364) obviously did not result in more complaints of fatigue. 


\section{conclusions}

\section{The Inventory of Subjective Health}

The 2 studies (chapter 1 and 2) showed a good association between the scores of the Inventory of Subjective Health on the one hand and perceived health rates, side effects, dropouts and patients' drug choices on the other hand. This relationship supports the validity of the Inventory of Subjective Health as a measure of general well-being, also during antihypertensive treatment.

The placebo controlled study with nebivolol showed a statistically significant improvement of the ISH-score versus baseline for both nebivolol and placebo. As a consequence this study underlines that an absolute change in quality of life perception cannot be claimed by comparison with baseline, since participation in a trial already influences quality of life perception.

\section{quality of life during antihypertensive treatment}

At the dosages used, nebivolol, bisoprolol and enalapril were effective anthypertensive drugs. Nebivolol, a B alter quality of life perception. The well-being scose during the highy selective $B_{1}$-blocker bisoprolol tended to be better than during the ACE-inhibitor cnalapril and more patients preferred to be treared with bisoprolol than with enatapril. These studies do not confirm the assumption that all s-blockers decrease quality of life and that ACE-inhibitors may preserve quality of life more than B-blocking agents, certainly not more than selective is 1 -blockers.

\section{exercise tolerance during antihypertensive treatment}

The reduction in endurance performance can influence quality of life of physically active patients. So far all antihypertensive drugs studicd, reduce endurance exercise time. However, a reduction of $8-12 \%$ - like with ACE-inhibitors and calciumantagonists - in general does not result in increased complaints of fatigue. In contrast the complaints of fatigue during B-blockade are very well known. The reduction in endurance perfomance depends on the selectivity of the B-blocker and the dosage used. Regarding nebivolol, a favorable effect of an ancillary property cannot be excluded. 


\section{PART IV \\ GENERAL DISCUSSION : \\ CURRENT ANTIHYPERTENSIVE THERAPY AND ISSUES FOR FUTURE RESEARCH}

The goal of antihypertensive treatment is prevention of the ill effects of the elevated blood pressure without impairing quality of life or inducing other cause morbidity and mortality.

Since target-organ damage is associated with marked increases in risk for people with hypertension and since evidence indicates that these aggravated risks are only partially reversibie by treatment, a key task is the early detection of high blood pressure - before target-organ damage has occurred - and sustained, intensive, and comprehensive treatment with the objective to prevent the development of targetorgan damage (19). Apart from treatment of the elevated blood pressure, management of other risk factors for ill effects of hypertension, like smoking, dyslipidaemia, insulin resistance and emotional stress is helpful. In atherosclerotic disease also antithrombotic agents like aspirin can be useful.

In this part of the thesis current antihypertensive therapy is discussed.

\section{1. which blood pressure to treat?}

There is still a lot of debate about which level of raised blood pressure should be treated. On the one hand, studies such as the Australian National Blood Pressure Study (ANBPS; 374) did not provide convincing evidence to treat diastolic blood pressures lower than $100 \mathrm{mmHg}$. An explanation could be some noxious effect of the drugs, or a lower than expected complication rate in mild hypertension. On the other hand, meta-analysis of nine major trials relating to mild hypertension has demonstrated a $34-40 \%$ lesser incidence of stroke in treated than in control hypertensive patients, while reductions in nonfatal events averaged $10 \%$ and fatal events $8 \%$, neither change being statistically significant (375).

\section{2. how far should blood pressure be lowered?}

There is no consensus that the aim of treatment in patients with hypertension is maximal lowering of blood pressure (376), either because little may be gained beyond a certain level in terms of risk reduction or, more seriously, because risk may increase at lower levels of treated blood pressure $(171,377-382)$. Due to the increased mortality risk of treated diastolic blood pressure below $85 \mathrm{mmHg}$ observed in different studies $(171,377-382)$, the treated blood pressure-risk relation forms a J-shaped curve. However, such a J-shaped curve is not seen in other studies, especially when elderly patients are excluded $(177,376)$. But, in patients with coronary heart discase cardiovascular events were increased at treated diastolic blood pressures below $85 \mathrm{mmHg}$ (383). Since diastolic pressure determines coronary perfusion pressure, a low diastolic pressure might be critical for the oxygen supply in patients with cardiac ischaemia. The strongest evidence against a treatment-induced causal relation comes from the Hypertension in Elderly Patients trial, in which an increased incidence of coronary heart disease was associated with the lowest diastolic pressures $(<80 \mathrm{mmHg})$. But this increased 
risk at the lowest pressures was found in both patients given active treatment and in the untreated controls (383). Thus, it seems that the J-shaped curve exists especially in elderly patients and the increased risk at the lowest blood pressures is not due to the antihypertensive therapy.

There is strong evidence from analysis of large trials in the elderly that $\mathrm{J}$ curves are due to the fact that preceding illness lowers blood pressure rather than that lowering the blood pressure precipitates death from coronary heart disease (376). For example, [1] a decrease in blood pressure after myocardial infarction is associated with poor survival (384), Also [2] the high pulse pressure - due to stiff large arteries is a plausible explanation; subjects with a high systolic pressure and low diastolic pressure are at greater risk for cardiovascular events than the general population (385). A third of the patients in the Systolic Hypertension in the Elderly Program trial had levels of treated blood pressure in this category (isolated systolic hypertension). A reduction in pressure by active treatment reduced the risk (376).

On balance, blood pressure can be decreased without danger to levels of 125/85 $\mathrm{mmHg}$ (376). A further decrease might be beneficial. Although many observations indicate that most of the increased risk of the J curve is due to the confounding effect of concomitant illness which lowers blood pressure, it cannot be ruled out that a low diastolic blood pressure might compromise coronary flow in some patients with advanced coronary heart disease.

\section{3. which antihypertensive drug to choose?}

\section{1. rationale.}

\subsection{1. effect on risk factors as a discriminating criterion?}

Regularly the choice of antihypertensive drugs is argued on the basis of their effects on risk factors for coronary heart disease. A recent externsive report on this issue has been published by Houston (386). In this article the influence of different anthypertensive drugs on 18 risk factors (Table 4.1) was mentioned. In addition, the author calculated a relative risk ratio for each antihypertensive drug by adding the number of known negative influences.

Table 4.1. Cardiouascular risk factors as publisbed by Houston (386)

\begin{tabular}{|l|l|l|}
\hline hypertension & dyslipidaemia & glucose intolerance \\
\hline exercise & potassium & insulin resistance \\
\hline magnesium & uric acid & leat veniricular hypertrophy \\
\hline blood viscosity & blood velocity & catecholamines \\
\hline mgiotensin li & antiatherogenic & arrhythmia potential \\
\hline platelet function & ibrinogen & thrombogenic potential \\
\hline
\end{tabular}

Apart from the fact that influences mentioned in this article are not always in accordance with other literature data and that no difference has been made between selective and nonselective ß-blockers and calcium-antagonists, also several methodological critiques can be made. [1] It is very unlikely that each listed risk factor should represent a same "risk weight" for coronary heart disease, which is an obligatory condition for simple addition of different risk factors. [2] Risk factors are often not independent. For example, blood viscosity is largely determined by the serum 
fibrinogen. level. [3] The calculation of the risk ratio did not account for positive or unknown influences. [4] Our knowledge of risk factors is not likely to be complete. For example, little is known about why some patients with high cholesterol levels, or smokers develop less atherosclerosis than others. As a consequence, a risk ratio from an incomplete list might result in completely aberrant results. $\lceil 5 \mid$ The clinical significance of drug-induced changes still remains unclear (387).

Different risk calculation formulae have been proposed. For example, the ischaemic heart disease risk formula of Gordon (see appendix; 388), which has been applied to the TAIM study (389), was recently published. Such formulac are clearly more valid than a simple addition of risk factors, since they are derived from large epidemiological studies, such as the Framingham Study (388). But, also these formulae do not face all of the aforementioned critiques, and certainly not the question whether the risk calculation also can be applied to drug-induced changes in risk factors

Appendix: risk for ischamic beart disease oter the next 8 years.

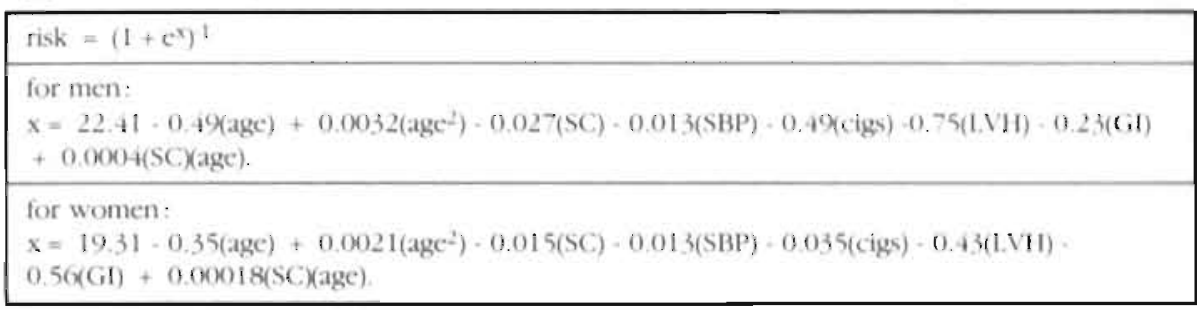

SC: serum cholesterol (mg/d); SBP : systolic blood pressure (mmHg), cigs : cigaretes (cnice I for : smoker, else 0); LVH : left ventricular hypertrophy (enter I if diagnosed by electrocardiogram, else 0), Gl: glucose intolerance (enter 1 if diabetic. casual blood sugar is over $6.7 \mathrm{mmol} / 120 \mathrm{mg} / \mathrm{dl}]$, or trace or more sugar in the urine, else $(0)$.

Indeed, there might be substantial doubt about the clinical significance in terms of morbidity and mortality of the observed drug-induced changes. For example, B-blockers (especially nonselective) may induce an unfavorable lipid profile. Total cholesterol does not change significantly, but triglycerides increase and, maybe more important, in several studies HDL was significantly decreased with nonselective is-blockers (390). In contrast, the nonselective s-blockers with intrinsic sympathomimetic activity appear to have less effect on the lipid profile than those without ISA. Paradoxically, despite the potentially adverse changes in the lipid profile, the B-blockers without ISA, and not those with ISA, provide a persistent reduction in mortality after myocardial infarction (391). Therefore, I have to conclude with Cruickshank (392) that the use of surrogates for coronary heart disease, e.g. coronary risk factors such as plasma lipids, blood sugar and insulin resistance can be dangerously misleading.

\subsection{2. major criteria to make a choice.}

[1] Most important is the effect of antihypertensive therapy on morbidity and mortality, the so-called hard endpoints. Since in hypertension heart disease is much more common $(30,177)$ and less decreased than stroke by antihypertensive treatment (177), drugs preventing heart disease might bring more progress. In addition, results from interventions with lipid lowering drugs - as discussed in part I - 
have taught that drug interventions have to be judged on all cause morbidity and mortality and not on cardiovascular morbidity and mortality alone (239). [2] The effect on quality of life is also very important because it cannot be the aim of therapy to impair quality of life for years and decades in a whole population of asymptomatic patients, while only a part of them might benefit from therapy. The cure should never be worse than the disease. In addition, poor patient compliance with therapy can substantially influence hard endpoints. Compliance may be substantially influenced by drug-induced changes in quality of life (187). 13] Concomitant conditions and diseases may also determine the choice of drug. $[4]$ In the absence of hard endpoints beneficial effects remain speculative. Very careful consideration of the influence on some risk factors might reduce the speculative error of the choice. Cardiac hypertrophy [1] seerns to be a key risk factor (part I chapter 2). Other important risk factors might be: progressive atherosclerosis (part I chapter 2), especially when [2] rupture prone weak cholesterol-rich plaques are present, [3] physical stress on large arteries (part II chapter l) - including stress frequency - and 14] a thrombogenic state (part I chapter 2). Serum cholesterol, glucose intolerance and smoking might increase the risk for these atherosclerosis-associated conditions.

\section{2. effect of antihypertensive drugs on criteria for drug choice.}

\subsection{1. diuretics}

effect on bard endpoints. Diuretics are effective against stroke, as shown by different studies such as the Australian National Blood Pressure Study (ANBPS; 393) and the Veterans Administration Cooperative Study (394).

In middle-aged males diuretics had a higher mortality from coronary heart disease than the untreated group (Oslo Study;395) and s-blocker therapy (MRC and IPPSH, Maphy; 396,397). Also the ANBPS study did not provide evidence for CHD prevention.

bard endpoints in subgroup. In contrast, in elderly hypertensive patients diuretics decreased not only stroke but also incidence and mortality from coronary heart disease (EWPHE study, SHEP study; 398,399). The MRC trial of treatment in older adults confirmed that diuretics, significantly reduced the frequency of myocardial infarction $(400)$. In this trial they were even better than is-blocker therapy.

quality of life. A metaranalysis on the effect of antihypertensive drugs on quality of life (401) showed that average score on general well-being with diuretics was worse than with ACE-inhibitors, calcium-antagonists and B-blockers. But diuretics were better than centrally acting $\alpha_{2}$-agonists.

\subsubsection{ACE-inhibitors}

bard endpoints. To date, no morbidity or mortality data are available for hypertensives treated with ACE-inhibitors.

bard endpoints in subgroup. In patients with sysiolic cardiac dysfunction and overt heart failure ACE-inhibitors lower mortality (CONSENSUS I, SOLVD, V Heft II, SAVE; 67-68,193-194).

speculative beneficial effects.

ACE-inhibitors are also more powerful than other antihypertensive drugs in reducing left ventricular hypertrophy (191), It is not clear whether they are superior to other antihypertensive drugs in primary prevention of cardiac hypertrophy and heart falure in hypertension. 
They have potential anti-arrhythmic effects (ischaemic and postinfarction antiarrhythmic effects have been shown in animal models) and may reduce infarct size (402-405). They might however disturb the cardiac repair process after myocardial infarction (197). In man the lower mortality in patients with cardiac dysfunction during ACE-inhibitor therapy was related to a reduction in the number of sudden deaths (CONSENSUS). This anti-arrhythmic effect might be due to an improved cardiac function, correction of hypokalaemia and hypomagnesiacmia and of the increased levels of catecholamines.

ACE-inhibitors also reduce insulin resistance (406). Diabetics might benefit from ACE-inhibitors, since they improve insulin sensitivity and possibly renal function (406-407).

quality of life. ACE-inhibitors are, in general, well tolerated. But, in contrast to what is generally assumed, they are not superior to selective $\aleph_{1}$-blocking agents in preserving quality of life (part III chapter 2, 341,401,408).

\subsection{3. calcium-antagonists}

bard endpoints. To date no data on primary prevention of morbidity or mortality are available for hypertensives treated with calcium-antagonists. Non-dihydropyridine calcium-antagonists like verapamil and diltiazem, probably reduce mortality after myocardial infarction. But unlike B-blockers, this effect is only present in patients with a good left ventricular function (DAVIT II, Diltiazem Postinfarcton Trial; 217-218).

The INTACT study (207) showed a significant reduction in new coronary lesions as compared with placebo, in postmyocardial infarction patients. But mortality with nifedipine $[\mathrm{n}=12]$ was higher than with placebo $[\mathrm{n}=2]$. Dihydropyridine calcium-antagonists are associated with a significant excess of myocardial reinfarction and also with death in the peri-infarction period or in chronic angina (409). A study on chronic angina, published by the United States Food and Drug Admin= istration mentioned a significant $63 \%$ excess of cardiovascular cvents in the calcium-antagonist studies in comparison with a $24 \%$ decrease in events in B-blocker studies (410). Among the possible reasons for this finding are increased heart rates and increased levels of angiotensin II and carecholamines (392). This hypothesis has been supported by the results of the HINT trial (411). This trial indicates that the dihydropyridine calcium-antagonist nifedipine, added to a cardioselective B-blocker, may have a beneficial effect on cardiac ischaemia, while nifedipine alone increased the risk of myocardial infarction.

quality of life. Calcium-antagonists were, on average , somewhat less positive in the preservation of general well-being (401). However, in this meta-analysis no difference has been made between selective and nonselective calcium-antagonists. Dihydropyridines like nifedipine, as monotherapy, are not very well tolerated (412). A recent quality of life study showed that nifedipine retard was associated with more symptomatic complaints and higher discontinuation rate than the ACE. inhibitor cilazapril and the B-blocker atenolol (408). Side effects of dihydropyridines are vasodilation-related side effects like tachycardia, headache, flushing and oedema (125). Most of these side effects are most predominant at peak plasma level. They can be diminished by decreasing the rate of change in plasma level (413). In this respect calcium-antagonists with a long half-life or controlled release: preparations (414) might be of interest. 


\subsection{4. $\alpha$-blockers}

hard endpoints. There are no data on hard endpoints. speculative beneficial effects.

They improve the blood lipid patterns, insulin resistance and the calculated risk for CHD (228,415). In hypertensive patients doxazosin reduced platelet aggregation (416), improved fibrinolytic activity and increased tissue plasminogen activity (417). Therefore, they are expected to retard atherogenesis and they might help to prevent acute coronary syndromes.

quality of hife. No controlled quality of life studies with $\alpha$-blockers are available. The main disadvantage is a tendency for postural hypotension. Apart from first dose hypotension and syncope, also during chronic therapy orthostatic hypotension may occur. Orthostatic hypotension is reported in up to $20 \%$ of patients, while syncope occurs in less than $2 \%(168)$. Orthostatic side effects are less frequem in second generation $\alpha$-bjockers like doxazosin and terazosin than during prazosin (168). Elderly patients are more susceptible to this side effect (125). Since elderly have more osteoporotic bones, a syncope or dizziness might result in life threatening events such as a hip fracture.

\subsubsection{S-blockers}

hard endpoints. B-blockers with ISA are not effective in reducing mortality after myocardial infarction (391). But, B-blockers without ISA are very effective in preventing stroke in both young and middle-aged patients with mild-to-moderate hypertension (MRC, MAPHY/HAPPHY, IPPPSH; 323,397,418) and elderly patients (Coope; 419).

In middle-aged men with severe hypertension atenolol-based treatment reduced mortality from myocardial infarction (420). Not only in hypertensives but also in normotensive patients, several B-blockers including propranolol, timolol, and metoprolol have been shown to reduce total and CHD mortality significantly after myocardial infarction (42 l-424). B-blockers were more effective than diuretics in preventing sudden death (MRC; 425) and myocardial reinfarction (Wikstrand, MAPHY/HAPPHY, MRC; 396-397,425).

A pooling study by Cruickshank (426) of all is-blocker trials in hypertension, including retrospective, prospective, controlled and uncontrolled trials suggested that R-blockers conterred a modest degree of primary prevention from myocardial infarction of $15 \%$ when compared with placebo, limited to nonsmoking men. This is due to the fact that nonselective ß-blocking agents were only beneficial for CHD in nonsmokers, while $B_{1}$-blockers were effective in both smokers and nonsmokers (397). Not only CHD mortality but also all-cause mortality decreased $(323,420)$.

In addition, s-blockers reduce the late morning peak incidence of myocardial infarction (81) and also reduce the likelihood of full-thickness myocardial infarction (427). In the peri-infarction period and 28 days after infarction B-blockers reduce mortality in patients with good and poor left ventricular function $(391,428)$. bard endpoints in subgroups. The effects in the elderly is not so clear. Two studies did not find a beneficial effect of atenolol on coronary heart disease (MRC, Coope; 400,419), while in the recent SHEP study (399) beneficial effects may have been associated with the presense of atenolol with a low-dose diuretic.

speculative beneficial effects. In animais, B-blockers have also proved effective in preventing coronary atheroma in spite of \&-blocker induced blood lipid changes 
(246). B-blockers have also a weak antiplatelet action, which might help in the prevention of thrombus formation $(429)$.

B-blockers are moderately effective in reversing left. ventricular hypertrophy (191), although dual acting agents (e.g. nebivolol) that reduce peripheral resistance and increase vascular compliance may be superior (392).

The negative inotropic and chronotropic effect of B-blockers could be vital components of their life-preserving quality $(430)$. These effects reduce the stress on the coronary arteries, thereby decreasing the risk of plaque rupture, which results in less progression of atherosclerosis and less "acute cardiac events". An association between heart rate and cardiovascular mortality has indeed been found in the Framingham study (431). Recently also clinical evidence of a beneficial effect of B-blockers in heart failure has been reported (432).

quality of life. The aforementioned meta-analysis indicated that B-blockers together with ACE-inhibitors were the best in preserving general well-being (401). However, in this analysis no difference has been made between nonselective and selective B-blockers. Nonselective B-blockers are not very well tolerated $(341,433)$, while recent studies have shown that $B_{4}$-blockers are as well tolerated as ACEinhibitors $(341,401,408)$ and highly selective B-blockers like bisoprolol might even be better tolerated (part III. chapter 2). Also the selective B,-blocker with vasodilating properties nebivolol preserved quality of life (part III chapter 1).

\section{3. influence of concomitant diseases or conditions on the choice of antihypertensive drugs.}

One of the most important determinants for selecting antihypertensive therapy is the presence of concomitant medical conditions and resulting use of medications (434). A concomitant disease can make the use of a drug less suitable or in contrast more appropriate. For example, B-blocker therapy can enhance bronchospasm in patients with obstructive pulmonary disease. Therefore, a B-blocker should not be: used in this condition ( 434 ).

A concomitant disease may favour the choice of drugs, which exert a beneficial effect on the concomitant clisease. For example, in hypertensive patients with angina pectoris, B-adrenoceptor antagonists and calcium channel blockers may be: firstline drugs (455). If the hypertensive patient also suffers from supraventricular arrhythmia, non-dihydropyridine calcium-antagonists or B-blockers might be helpful (436). Diuretics have proven their validity in treatment of congestive heart failure (437). Recently also ACE-inhbitors have shown to reduce mortality in this condition $(67,68,194)$. Studies with other antihypertensive drugs like selective calcium-antagonists and B-blockers are ongoing.

Concomitant therapy can also influence the choice of the drug. Elderly patients, for example, often suffer from arthritis (438), which can be treated with nonsteroidal anti-inflammatory drugs. However, these drugs can cause sodium retention. As a consequence, in this condition often a diuretic will be required (435).

Apart from concomitant diseases and their therapy, also some conditions of the patient can influence the choice of the antihypertensive agent. For example, in the elderly, diuretics have proven their validity (398-399) and might therefore have some preference above other antihypertensive drugs in these patients. Due to the reduction in exercise tolerance, nonselective $B$-adrenoceptor antagonists can better be avoided in physically active patients (part III, chapter 3 ). 


\section{4. monotherapy or low-dose combination therapy?}

Controversy exists as whether it will be preferable to increase drug doses or add or substitute other drugs if inadequate antihypertensive effects are not achieved. Since side effects mostly increase with increasing doses of a drug, in general, it may be preferable to add another drug when a partial response with good clinical tolerance to the first drug is achieved. Likewise, the substitution of another drug may be preferable when there is litte or no antihypertensive response or if adverse drug effects ensue due to the first agent (439). In this philosophy of low dose therapy and rapid combination of drugs, multiple action drugs gain interest.

\section{5. a personal view on current treatment of hypertension}

Most progress in the treatment of hypertension can be made by preventing the ill effects of atherosclerosis. Ideally atherogenesis itself should be prevented or slowed down. However, our knowledge on the pathophysiology and especially the treatment of atherogenesis is still insufficient to justify large and costly detection and intervention programs for early detection and treatment of atherosclerosis. Primary prevention of atherosclerosis and hypertension nowadays should be based on health education programs, dissuading people from smoking and promoting dicts with less fat and less saturated fatty acids, a lower sodium potassium ratio, and maybe also higher content of antioxidants. Also regular moderate physical exercise, alcohol restriction to 2 consumptions per day, and avoidance of overweight and excessive emotional stress (e.g. type A behaviour) should be advised. Early in atherogenesis, calcium-antagonists might become more frequently used to retard atherogenesis, providing they are well-tolerated and do not induce other morbidity or mortality.

Since early prevention of atherogenesis is not yet possible, our efforts should go to the prevention of the ill effects of atherosclerosis, which are mainly due to plaque rupture and thrombus formation. Therefore, if atherosclerotic plaques are present, aspirin (or in some cases a thromboxane synthetase inhibitor/receptor antagonist) could be used to prevent platelet aggregation. Risk factors for plaque rupture are weak (e.g. cholesterol-rich) plaques and physical stress on the vessel wall (systolic blood pressure, pulse pressure, hean rate, ardiac inotropy, and probably also shear stress). Since lipid lowering interventions did not improve all cause mortality and even might induce a more aggressive behaviour, these interventions should be restricted to patients who are at particularly high risk for death from coronary disease: those with substantially elevated serum cholesterol concentrations, history of coronary disease, or several risk factors in addition to hypercholesterolaemia (part I chapter 2).

Which antihypertensive drug to choose? Such a choice has to be made for each individual patient, but a preference drug(s) for the population of hypertensive patients can be considered. Since several drug-induced "speculative beneficial and adverse effects" have not fulfilled expectations on hard endpoints (see 3.1.2), in my opinion, anthypertensive therapy of choice should predominanty be based on the secure hard endpoints and effects on guality of life with a smaller weight for speculative effects. It is clear that the antihypertensive therapy of choice is based on current knowledge and is likely to change as knowledge progresses.

Nowadays, nwo classes of antihypertensive drugs seem the best in preserving quality of life: selective $B_{1}$-blockers and ACE-inhibitors. In addition, B-blockers have 
been shown effective in primary and secondary prevention of coronary heart disease, and also all cause mortality was improved. Therefore, selective $B_{1}$-blockers are in general the drugs of choice - certainly in middle-aged hypertensive patients without interfering illness. If ACE-inhibitors prove to have similar favorable effects on mortality, they may develop as alternative first choice drugs.

Further investigation is needed to confirm the beneficial effect of is-blockers in the elderly. In these patients the beneficial effects on hard endpoints are available for diuretics. It is very likely that, with ongoing research, also other anthyperiensive drugs might provide more data on hard endpoints (cardiovascular and all cause morbidity and mortality). However, $B_{1}$-blockers also possess several additional speculative beneficial effects (see 3.2.5).

$B_{1}$-selective blockers with vasodilating properties are promising since compared with other B-blockers they reduce the afterload. for the heart. This might be an additional beneficial effect in preventing cardiac hypertrophy. In addition, some of these B-blockers may preserve qualiry of life and - important in physically active patients - in contrast to other B-blockers do not substantially alter exercise performance (part III chapters 1 and 3).

\section{6. individualizing therapy}

Despite several decades of innovative research that has elucidated behavional and biological risk factors for coronary heart disease $(440)$, the ability 10 identify individuals who will eventually suffer acute thrombotic events is incomplete (441). In the United Kingdom Heart Disease Prevention Project (442), for example, highrisk patients identified by hypertension, smoking, and clevated blood cholesterol levels accounted for only $32 \%$ of all future infarctions. In addition, we have to bear in mind that results of trials are means of a population. There are patients doing better, and other patients doing worse. As a consequence, recommendations derived from trials will suit for the majority of patients, but not for each individual patient. Some individualization of therapy can be made by correcting for concomitant risk factors or diseases, but the black box with lack of knowledge about the "patient to treat" remains considerable. Measurement of cardiac mass, vessel wall properties and vessel wall structure might provide more information about the risk of the individual patient. Such measurements might be more helpful for the management of hypertension in the individual patient than current risk calculations.

\section{7. issues for future research}

\section{1. evaluation of current antihypertensive therapy}

Long-term prospective clinical trials are needed to compare morbidity and (all cause) mortality with ACE-inhibitors, calcium-antagonists, and $\alpha$-blockers to that of diuretics and the different subclasses of B-blockers (especially the $B_{1}$-blockers without ISA and those with vasodilating properties).

\section{2. prevention of ill effects}

Primary prevention of atherosclerotic complications remains the most important of the unresolved issues in antihypertensive therapy (387). Since atherosclerosis already starts at puberty, prevention has to start early in life. As long as atherogenesis cannot be prevented, much attention should be paid not only to 
progression and regression of atherosclerosis but also to prevention of plaque rupture by stabilizing weak plaques and by reducing the stress on these plaques.

As a consequence, much more attention should be paid to the role of physical factors - like circumferential and shear stress - in the pathogenesis of ill effects. Important issues for future research are effects of changes in local wall tension, determination of the relative impact of different stress factors of the pulsatile stress and impact of mean and pulsatile shear stress. Heart rate and pulse pressure are two vessel wall stress factors (part II, chapter 1). These factors might not only be important for large arteries. In a recent study Christensen found that $80 \%$ of the mediatumen ratio of resistance vessels in rats could be explained by the pulse pressure and heart rate, with the mean pressure playing but a small role (443). Long-term prospective studies are needed correlating different aspects of vessel wall stress with morbidity and mortality in hypertension.

\section{3. reversal of ill effects}

Reduction of blood pressure may result in reduction of some pressure related ancrial damage, but until now reversal of hypertension with drugs may not cause reversal of the hypertrophy and collagen content (380). Reversibility potential of organ damage should be further investigated.

Growth factor inhibitors, like ACE-inhibitors and possibly also nonselective HMG co-A reductase inhibitors like simvastatin (444), might be promising not only to prevent but also to reduce hypertrophied structures more rapidly. However, when eutrophy has been reached, do these drugs maintain this eutrophic state or are they inducing (cardiac and/or vascular) atrophy? In other words, can these drugs be used for a long indefinite period or are these drugs only for initial therapy, followed by other therapy when euthrophy has been reached? In addition, there is still little direct evidence that the altered vascular structure in essential hypertension is associated with vascular growth. There is more evidence that it may be associated with remodeling (rearangement of existing cells), as suggested by Mulvany, Baumbach and Heistad (445-446). This suggests that if anthypertensive treatment is to correct the vascular structure, it is possible that the treatment should be directed not so much at preventing growth, but more at facilitating rearrangement of existing cells (445).

\section{4. around the clock protection}

The effect of an antihypertensive therapy not only depends on the intrinsic efficacy' of a drug, but also on its ability to provide a good around the clock protection. This largely depends on drug adherence which is also influenced by the effect of therapy on quality of life. Investigation of the influence on quality of life should be continued. Not only the effect of different classes of antihypertensive drugs should be compared but also drugs within the same class, e.g. dihydropyridine calcium-antagonists with different half-lives.

Even with satisfactory drug adherence, current antihypertensive therapy often does not protect the early morning hours sufficiently without risk for hypotension during the night. Further development of drug delivery systems might resolve this problem. 


\section{5. identification of patients at risk}

Very important from a socio-economic point of view is the identification of patients at risk. Therefore, considerable efforts should be made to better assess the risk not only in the population but especially in the individual patient. In this respect cardiac hypertrophy, heart rate, vessel wall structure and properties might be key standards. New techniques to detect early stages of atherosclerosis and rup. ture prone small plaques should be developed. Intravascular echo is a step forward in this respect, but because of its invasive nature, it is not suitable for population screening.

\section{6. controlling drug exposure}

Poor compliance is a problem of drug therapy in general and not of antihypertensive therapy in particular, but its consequences for outcome of therapy may be very important. Compliance in hypertensive patients and methods to improve it are discussed in part 1, section 1.2.4. Poor compliance may also decrease the power of clinical trials (447). It has been calculated that if $10 \%$ of patients in a trial are noncompliant, the power of the study would be reduced from 0.95 to 0.90 , and if $30 \%$ are noncompliant, the number of patients has to be doubled $(448,449)$. In addition, if compliance is different in the 2 limbs of a comparative study, false conclusions about efficacy and side effects can be made (450).

Compliance has been measured by different methods. Patient report reveals only a fraction of the non-compliers (447), since 'patients often lie when they state they have taken certain medicines' (Hippocrates) to please the clinician or to avoid disapproval (451). Pill counts are frequently used in clinical trials since Good Clinical Practice guidelines ask for drug accountability (447). Pill counts are more reliable than patient report (452), but can grossly overestimate compliance (448), and misses some $10 \%$ of overt non-compliers (447). Pill counts only can indicate poor compliers if the amount of pills returned is obviously too large. It never can prove good compliance, since some non-compliers may discard tablets before returning containers $(447,451)$. This "pill dumping" has to be suspected if less pills are returned than expected (453). Drug plasma levels can prove that drug has been taken, but in general cannot detect the 'toothbrush' phenomenon, when noncompliers resume full compliance or more one or a few days before control visits $(451,454)$. Recently, two interesting methods have been developed to monitor drug adherence more adequately: the long half-life, low-dose chemical markers and the micro-electronic medication event monitoring system (MEMS). As low-dose markers phenobarbital ( $2 \mathrm{mg} /$ day) and digoxin ( $2.2 \mathrm{~g} /$ capsule) have been used ( 448 , 452). Phenobarbital has the advantage to give reproducible steady-state plasma levels with little variation between peak and trough levels (455) and without large interindividual variation (456). These markers can monitor drug adherence for the last days to weeks (448), but give no precise information on the time of dosing. With low-dose digoxin monitoring the proportion of good compliers was equal to those who have returned less than $15 \%$ of the capsules. Combination of digoxin monitoring with pill counts improved compliance control (452). The MEMS monitor registers every opening of the container with accurate time registration, but cannot prove drug intake (451). The fact that methods of compliance measurement usually have to be explained to patients prior to the study as a part of informed consent (447), can decrease the validity of pill counts and MEMS devices. 
From the results of these newer compliance monitoring methods used in 4 clinical trials, Urquhart estimates that 50 to $70 \%$ of patients will take greater than $80 \%$ of the prescribed doses, 30 to $40 \%$ will take 40 to $80 \%$ of prescribed doses, and a small percentage will take, respectively, 100 to $120 \%$ or less than $40 \%$ of prescribed doses (456). In a subset of the Helsinki Heant Study $31 \%$ of patients were identified as poor compliers (452). With MEMS and low-dose long half-life markers it was also confirmed that compliance with once daily regimens was slightly better than with BID, but markedly better than with TID or QID regimens $(457,458)$. In different shortoterm trials using once or twice daily regimens compliance averaged more than $80 \%$ of pills taken $(457,459)$, suggesting that noncompliance is also stimulated by chronic treatment.

Altough recently much progress in controlling drug adherence has been made, a gold standard is not yet available. Combination of different methods can improve compliance control (452). Efforts should be made to further improve methods of controlling compliance. Compliance should be considered as a variable in the analysis of trial data. If appropriate, a separate analysis on data of compliers should be made (447), 


\section{ACKNOWLEDGEMENTS}

The studies presented in part II, chapter 3, and part III chapters 1 and 3 were supported by a grant of the Janssen Research Foundation.

The logistic participation of the physicians M. van Bommel, J. Screever and J. Zuidweg in these studies is gratefully acknowledged.

The study presented in pant III chapter 2 was supported by a grant of the E. Merck company. The participation of the following investigators: J. Joosten, dept of Medical Sociology, University of Limburg, Maastricht; F. Lustermans, dept of medicine, De Wever Hospital, Heerlen; J. Smit, dept of medicine, Diakonessen Hospital, Naarden; L. de Vries, dept of medicine, De Sionberg Hospital Dokkum, is very much acknowledged.

Verapamil and placebo tablets (part II chapter 2) were kindly provided by Knoll $\mathrm{BV}$.

The expert technical assistance of Mrs A. Baisier, Mrs J. Bost, Mrs M. Fuss, Mrs A. Jacobs, Mrs M. Joris, Mrs M. Sysmans, Mrs A. Wuyts and the secretarial assistance of Mrs E. Geurts and Mrs M. Hogenboom are gratefully acknowledged.

Last but not least, I want to thank Prof Dr J.D. Fitzgerald and Prof Dr J. Urquhar for their expert advice on different studies. 


\section{REFERENCES}

1. Safar ME. Pulse pressure in essential hypertension: clinical and therapeutical implications. J Hypertens 1989, 7.769-776

2. Ferguson IJ. Randall OS. Hemodynamic correlates of arterial compliance. Catheterization and cardiovascular diagnosis 1986, 12:376-380.

3. Nakayama R, Azuma T. Noninvasive measurements of digital arterial pressure and compliance in man. Am J Physiol 1977, 2:H168-H179.

4. Guyton AC. Blood pressure control : special role of the kidneys and body fluids. Science 1991, $252: 1813-1816$

5. Cowley AW. Long-term control of arterial blood pressure. Physiol Reviews 1992, 72:231-300.

6. Guyton AC. Kidneys and fluids in pressure regulation. Small volume but large pressure changes Hypertension 1992, 19(suppl 1):12-18.

7. Hollander W. Hypertension, antihypertensive drugs and atherosclerosis. Circuiation 1973. $48: 1112-1127$.

8. Joint National Committec. Hypertension prevalence and the status of awareness, treatment, and control in the United States. Final Report of the subcommittee on definition and prevalence of the 1984 Joint National Committee. Hypertension 1985, 7:457-468.

9. Williams GH, Braunwald E. Hypertension. In: Harrison's principles of internal medicine. Braunwald E, isselbacher KJ, Petersdorf RG, Wilson JD, Martin JB, Fauci AS (ects), McGraw-Hill, New York, XIth ed. 1987, ppl024-1037.

10. Pickering G. High Blood Pressure. J\&A Churchill Ltd, London, 2nd ed. 1968.

11. Ledingham JJG. Secondary hypertension. In: Oxford textbogk of medicine. Weatherall DJ, Ledingham JGG, Warrell DA (eds). Oxford University Press, Oxford, New York, Toronto, 1985. pp13.278-13.288.

12. Ferguson RK. Cost and yield of the hypertensive evaluation. Experience of a community-based referral clinic. Ann Intern Med 1975, 82:761-765.

13. Muller FB, Laragh JH. lssues, goals and guidelines for choosing first-line and combination antihypertensive drug therapy. In: Hypertension: pathophysiology, diagnosis and management Laragh JH and Brenner BM (eds), Raven Press New York, 1990, vol 2, pp2331.2342.

14. Ganten $D$, Takahashi ST, Lindpaintner $\mathrm{K}$, Mullins $\mathrm{J}$. Genetic basis of hypertension. The reninangiotensin paradigm. Hypertension 1991, 18:111109-III114.

15. Blaustein MP, Hamlyn JM. Pathogenesis of essential hypentension. A link between dietary salt and high blood pressure. Hypertension 1991, 18:III184-III 195.

16. Pickering TG. Does psychological stress contribute to the development of hypertension and coronary heart disease? Eur J Clin Pharmacol 1990, 39:SI-S7.

17. Hunt SC, Williams RR, Barlow GK. A comparison of positive family history definitions for defining risk of future disease. J Chronic Dis 1986, 39:809-821. 
18. Williams RR, Hunt SC, Hasstedt SJ, Hopkins PN, Wu L.L, Berry TD, Stalts BM, Barlow GK, Schumacher MC, Lifton RP, Lalouel JM. Are there interactions and relations between. genetic and environmental factors predisposing to high blood pressure? Hyperaension 1991, 18(suppl 1):129-137.

19. Stamler J. Blood pressure and high blood pressure. Aspects of risk. Hypertension 1991, 18(supp/1): 195.1107.

20. Slater EE. Insulin resistance and hypertension. Hypertension 1991, 18(suppl 1):1108-1114.

21. Defronzs RA. The effect of insulin on renal sodium metabolism. Diabetologia $1981,21: 165-171$

22. Kaplan NM. The deadly quartet : Upper-body obesity, glucose intolerance, hypertriglyceridemia, and hypertension. Arch Intern Med 1989, 149:1514-1520.

23. Reaven GM. Role of insulin resistance in human disease. Diabetes 1988, 37:1595-1607.

24. Willams RR, Hun SC, Hopkins PN, Stults BM, WU LL, Hasstedt SJ, Barlow GK, Stephenson SH, Ialoucl JM, Kuida 11. Familial dyslipidemic hypentension: Evicience from $58 \mathrm{U}$ tah families for a syndrome present in approximately $12 \%$ of patients with essential hypertension. JAMA 1988, $259 \cdot 3579.3580$

25. Foster DW. Insulin resistance: a secret killer. N Engl J Mcd 1989, 320:733-734.

26. Neumamn F. Waas W, Zimmermann R, Haupt H, Tillmanns H, Kubler W. Hacmorheologic studies in patients with reduced coronary vasodilator capacity but nomal coronary angiogram (Syndrome X). Eur lleart ] 1989, 10:509-513.

27. Sokolow M, Perlof D. The prognosis of essential hypertension treated conservatively. Circulation $1961,23: 697-713$

28. Kannel WB. Hypertension and the risk for cardiovascular disease In: Hypertension: pathophysiology, diagnosis and management Laragh JH and Brenner BM (eds). Raven Press New York, 1990, vol 1, pp101.117.

29. Fonhlich ED, Apstein C, Amstrong ML, Cohn JN, Cutler JA, Devereux RB, Levy D, Pettinger WA. Schulman NB, Walker WG. Target organ consequences in hypertension: parhogenesis and prevention. Hypericnsion 1991, 18(stippl 1):1143-1145.

30. Bulpitt C1, Beevers DG, Butler A. Coles EC, Hunt D, Munro-Faure AD. Newson RB, ORiordan FW, Petrie JC, Rajagopalan B, Rylance PB, Twallin G, Webster J, Dollery T. The survival of treated hypertensive patients and their causes of death: a report from the DHHS hypertensive care computing project (DHCCP). J Hypertens 1986, 4:93-99

31. Sleight P. Hypertension. In: Oxford Textbook of Medicine. Weatherall DJ, Ledingham JGG, and Warrell DA feds). Oxford Medical Publications, Oxford, 1985, pp13.258-13.278.

32. Foikow B. Physiological aspects of primary hypertension. Physiol Rev 1982, 62:347.504.

33. Bohlen iHG. The microcirculation in hypertension. J Hypertens 1989, 7:5117-5124.

34. Le Noble JLMI, Smith Tl. Hutchins PM, Siruyker Boudier HAJ. Microvascular alterations in adult conscious spontanecusly hypertensive rats. Hypertension 1990, 15:415-419.

35. Mentich HA, Romen W. Heimgärtner W, Hartung E, Băumer F. Capillary rarefaction characteristic of the skeletal muscle of hypertensive patients. Klin Wochenschr 1988, 66:54-60. 
36. Harper RN, Moore MA, Marr MC, Watts LE, Hutchins PM. Arteriolar rarefaction in the conjunctiva of human essential hypertensives. Microvasc Res 1978, 16:369-372

37. Schwarz SM, Campbell GR. Campbell JH. Replication of smooth muscle cells in vascular disease. Circ Res 1986, 58:427-444.

38. Lever AF. Slow pressor mechanisms in hypertension a role for hypertrophy of resistance vessels? J Hypertens 1986, 4:515:524.

39. Le Noble ILML. Tangelder GJ. Slaf DW, van Essen H. Reneman RS, Struyker Bondier IIA! A func tional morphometric study of the cremaster muscle microcirculation in young sponaneously hypertensive rats. I Hypertens 1990, 8:741-748

40. Struyker Boudier IiAJ. Van Bortel LMAB, De Mey JGR. Remodeling the vascular tree in hypertension: drug effects. TiPs 1990, 11:240-245.

41. Petrovitch H, Vogt TM, Berge KG. Isolated systolic hypertension: lowering the risk of stroke in oldẹ patients. Geriatrics 1992, 47:30-38.

42. Kannel WB. Castell WP, McNamara PM. McKee PA, Feinleib M. Role of blood pressure in the development of congestive hean failure. Framingham Study. N Engl J Med. 1972, 287:781-787.

43. Kannel WB, Wolf PA, McGee DL, Dawber TR, McNamara P, Casieli WP. Systobe biood pressure, arterial rigidity and risk of stroke. The Framingham Study. JAMA 1981, 245:1225-1229

44. Rutan GH, Kuller LH, Neaton JD, Wentworth DN, McDonald RU, McJate-Smitf. W., Mortality associated with drastolic hyperiension and isolated systolic hypertension among men sereened for Muftiple Risk Factor Intervention Trial Circulation 1988, 77:504-51.4.

45. Kannel WB, Dawber TR, McGee DL. Perspectives on systolic hypertension. 'Tlac Framingliam Study. Circulation 1980, 61:1179-1182.

46. Wing S, Aubert RE, Hanson JP, Hames CG, Slome C, Tyroler HA. Isolated systolic hypertension in Evans County. I. Prevalence and screening considerations. J Chronic Dis 1982, 35:735-742.

47. Casale PN, Devereux RB, Milner M, Zullo G, Harshfield GA, Pickering TG, Laragh JH. Value of echocardiographic measurement of left ventricular mass in predicting cardiovascular morbid events in hypertensive men. Ann Intern Med 1986, 105:173-178.

48. Grossman W, Braunwald E, Mann T, McLaurin L.P, Green LH. Contractile state of the left ventricle in man as elevated from end-systolic pressure volume relations. Circulation 1977, 56:845-852.

49. Levy BI, Safar M. Ventricular atterload and aortic impedance. In Swyngedauw B (ed.): Cardiac hypertrophy and failure, J Libbey INSERM London, Paris 1990 pp $521-530$.

50. Safar ME, Toto-Moukouo JJ, Bouthier JA, Asmar RE, Levenson JA, Simon AC. Arterial dynamics, cardiac hypertrophy and antihypertensive treatment. Circulation 1987, 75(suppl 1):156-161.

51. Bouthier JD, De Luca N, Safar ME, Simon AC. Cardiac hypertrophy and arterial distensibility in essential hypertension. Am Heart J 1985, 109:1345-1352.

52. Delcayre C, Swynghedauw B. Biological adaptation and dysadaptation of the hear to chronic arteral hypertension: a revicw. J Hypertens 1991, 9(suppl 2):S23-\$29.

53. Francis GS, MCDonald KM. Left ventricular hypentophy : an initial response to myocardial injury. Am J Cardiol 1992, 69:36.9G. 
54. Mercadier JJ, De La Bastie D, Ménasché P, N'Guyen Van CAO A, Bouveret P. Lorente P, Piwnica A, Slama R, Schwartz K. Alpha-myosin heavy chain isoform and atrial size in patients with various types of mitral valve: dysfunction: a quantitative study. J Am Coll Cardiol 1987, 9: 102+1030

55. Weber KT, Brilla CG, Janicki jS. Signals for the remodeling of the cardiac interstitium in systemic hypertension. J Cardiovase Pharmacol 199) 1, 17(suppl 2):S14-519.

56. Carver W, Nagpal. MI., Nachtigal M, Borg TK. Terracio L. Collagen expression in mechanically stimulated cardiac fibroblasts. Circ Res 1991, 69:116-122.

57. Weber KT. Brilla CG. Pathological hypertrophy and cardiac interstitium. Fibrosis and reninangiotensin-aldosterone system. Circulation 1991, 83:1849-1865.

58. Bauwens FR, Duprez DA, De Buyzere ML, De Backer TL, Kaufman JM, Van Hoecke J, Vermeulen A, Cement DL. Influence of the arterial blood pressure and nonhemodynamic factors on left ventricular hypertrophy in moderate essential hypertension. Am J Cardiol 1991, 68:925-929.

59. Brilla CG, Weber KT. Reactive and reparative myocardial fibrosis in arterial hypertension. Cardiovasc Res 1992, 26:671-677.

60. Guazi M, Fiorentin C, Olivari MT. Cardiac load and function in hypertension. Am J Cardiol 1979 , $44 \cdot 1007-1012$

61. Balogun MO, Durn FG. Systolic and diastolic function following regression of keft ventricular bypertophy in bypertension. J Hypertens 1991, 9(suppl 2):S51-\$55.

62. Giles T. Antihypertensive therapy and cardiovascular risk. Are all antihypertensives equal? Ilyperension 1992. 19(suppl 1):1124-1129.

63. Levy D, Garrison RI, Savage DD, Kannd WB, Castelli WP. Prognostic implications of echocardiographically determined left ventricular mass in the Framingham Heart Study. N Engl J Med 1900, $322: 1561-1566$

64. Cruickshank JM, Lewis J, Moore V, Dodd C. Reversibility of left ventricular hypertrophy by differ* ing types of antihypertensive therapy. I Hum Ilypertens 1992, 6:85-90

65. Mclenachan JM, Henderson E, Morris KI. Dargic HJ. Ventricular arthythmias in patients with hypertensive left ventricular hypermophy. N Engl ] Med 1987, 317:787-792.

66. Messẹli F11, Venura HO, Etizardi DJ, Dunn FG, Frohich ED. Hypentension and sudden death: increased ventricular ectopic activity in left ventricular hypertrophy. Am J Med 1984, 77:18-22.

67. The CONSENSUS Trial Study Group. Effect of enalapril on mortality in severe congestive heart failure: results of the Cooperative North Scandinavian Enalapril Survivai Study (CONSENSIS). N Engl ] Med 1987, 316:1429-1435.

68. The SOLVD Investigators. Effect of enalapril on survival in patients, with reduced left ventricular ejection fractions and congestive heart failure. N Engl J Med 1991, 325:293-302.

69. Ross $\mathbf{K}$. The pathogenesis of atherosclerosis - an update. $\mathbf{N}$ Engl J Med 1986, 314:488-500.

70. Fuster V, Badimon 1., Badimon J. Chesebro JH. The pathogenesis of coronary artery disease and the acute coronary syndromes (first of two parts). N Engl J Med 1992, 326:242-250.

71. Karino T, Goldsmith HL. Motomiya M, Mabuchi S, Sohara Y. Flow patterns in vessels of simple and complex geometries. Ann N Y Acad Sci 1987, 516:422-441. 
72. Glagov S, Zarins C. Giddens DP, Ku DN. Hemodynamics and atherosclerosis: insights and perspectives gained from studies of human arteries. Arch Pathol Lab Med 1988, 112:1018.1031.

73. Ip JH. Fuster V, Badimon L, Badimon J. Taubman MB, Chesebro JH. Syndromes of accelerated atherosclerosis; role of vascular injury and smooth muscle cell proliferation. I Am Coll Cardiol $1990,15: 1667-1687$.

74. Blankenhorn DH, Kramsch DM. Reversal of atherosis and sclerosis. The two components of atherosclerosis. Circulation 1989. 79:1-7.

75. Zarins CK, Bomberger RA, Taylor KE, Glagov S. Artery stenosis inhibits regression of diet-induced atherosclerosis. Surgery $1980,88: 86-92$.

76. Davies MI. Bland MJ. Hangartner WR, Angelini A, Thomas AC. Factors influencing the presence or absence of acute coronary thrombi in sudden ischemic death. Eur Heart J 1989, 10:205-208.

77. Nobuyoshi M, Tanaka M, Nosaka H, Kimura T, Yokoi H, Hamasaki N, Kim K, Shindo T, Kimura K. Progression of coronary atherosclerosis: Is coronary spasm related to progression? $\mathbf{j}$ Am Coll Cardiol 1991, 18:904-910.

78. Lin C-S, Penha PD, Zak FG, Lin JC. Morphodynamic interpretation of acute coronary thrombosis, with special reference to volcano-like eruption of atheromatous plaque caused by coronary artery spasm. Angiology 1988, 39:535-547.

79. Richardson PD, Davies MJ, Born GVR. Influence of plaque configuration and stress distribution on fissuring of coronary atherosclerotic plaques. Lancet 1989, 2:941-944.

80. Mulcahy D. Keegan 1, Cunningham D, Quyyumi A, Crean P, Park A, Wright C, Fox K. Circadian variation of total ischaemic burden and its alteration with antianginal agents. Lancet 1988 , ii 755.758 .

81. Muller JE, Stone PH, Turi ZG, Rutherford JD, Czcisler CA, Parker C, Pook WK, Passamani $S_{\text {: }}$ Roberts R, Robertson T, Sobel BE, Willerson JT, Braunwald E, and the MII.IS study group. Circadian variation in the frequency of onset of acute myocardial infarction. N Engl J Med 1985. $313: 1315-1322$.

82. Willich SN, Linderer $\mathrm{T}$, Wegscheider $\mathrm{K}$, Schroder R. Increased risk of myocardial infarction in the morning. J Am Coll Cardiol 1988, 11:28 (abstr).

83. Willich SN, Levy D, Rocco MB, Tofler GH, Stone PH, Muller JE. Circadian variation in the incidence of sudden cardiac death in the Framingham Study population. Am J Cardiol 1987. $60: 801-806$.

84. Muller JE, Ludmer PL, Willich SN, Toller GH, Aylmer G, Klangos I, Stone PH. Circachan variation in the frequency of sudden cardiac death. Cifculation 1987, 75:131-138

85. Millar-Craig MW, Bishop CN, Raftery EB. Circadian variation of blood pressure. Lancet 1978 , i: $795-797$.

86. Tofler GH, Brezinski D, Schafer AI, Czeisler CA, Rutherford JD, Willich SN, Gleason RE, Williams CH, Muller JE. Concurrent moning increase in platelet aggregability and the risk of myocardia] infarction and sudden death. N Engi J Med 1987, 316:1514-15!8.

87. Perralito A, Mangiafico RA, Gibiino S, Cuffari MA, Miano MF, Marzella C, Fiore CE. Daity modifications of plasma fibrinogen, platelet aggregation, Howells time, PTT, TT, and antithrombin III in normal subjects and in patients with vascular disease. Chronobiologia 1982, 9:195-201. 
88. Willich SN, Pohjola-Sintonen S, Bhatia S, Tofler GH, Shook TL, Muller JE, Williams GH, Stone PH. Morning increase of platelet aggregability in patients with coronary artery disease. I Am Coll Cardiol 1988, 11:20-iA (abstr).

89. Rosing; DR, Brakman P. Redwood DR. Blood fibrinolytic activity in man : diurnal variation and the response to varying intensities of exercise. Circ Res 1970, 27:171-184.

90. Andreotti F, Khan MI, deBart A, Hackett D, Davies GJ, Maseri A, Kluft C. Circadian lluctuations in endothelium derived fibrinolytic factors in relation to the time of onset of myocardial infaretion and sudden cardiac death. I Am Coll Cardiol 1988, 11:27A (abstr)

5). Falk E. Unstable angina with fatal outcome: dynamic coronary thrombosis leading to infarction and/or sudden death: autopsy evidence of recurrent mural thrombosis with peripheral embolizaton culminating in total vascular occlusion. Circulation 1985, $71: 699.708$.

92. Woolf N, Davies MI. Interfelationship between atherosclerosis and thrombosis. In: Fuster V, Verstraete M, eds. Thrombosis in cardiovascular disorders. Philadelphia: W. B. Saunders, $1992: 41-77$.

95. Falk E. Morphologic features of unstable atherothrombotic plaques underlying acute coronary syndromes. Am !Cardiol 1989, 63:1 !4E-120E.

9. Whilerson J1, Golino P, Eidi J. Campbell WB, Buja M. Specific platelet mediators and unstable coronary añeту leshons: experimental evidence and potential clinical implications. Circulation 1989. 80:198-205.

95. Webster MWI, Chesebro JH, Smith HC, Frye RI. Holmes DR, Recuer GS, Bresnahan DR, Nishimura, RA, Clements IP, Bardsley WT, Grill DE, Bailey KR, Fusier V. Myocardial infarction and coronary artery ocelusion : a prospective 5-year angiographic snidy. I Am Coll Cardiol 1990 , 15(suppl):218 A abstract.

96. Ambrose 1A, Tannenbaum MA, Aiexopoulos D, Hjemdahl-Monsen CE, Leavy J, Weiss M, Borrico $S$, Gorlin R, Fuster V. Angiographic progression of coronary artery disease and the development of myocardial infarcion. I Am coll Cardiol 1988, 12:56-62.

97. Ambrose 3A, Winters SL, Arora RR, Eng A, Riccio A, Gorlin R, Fuster V. Angiographic evolution of coronary artery morphology in unstable angina. J Am Coll Cardiol 1986, 7:472-478.

98. Liule WC, Constantinescu M. Applegate RJ, Kutcher MA. Burrows MT, Kahl FR, Santamore WP. Can coronary angiography predict the site of a subsequent myocardial infarction in patients with mild-to-moderate coronary antery disease? Circulation 1988, 78:1157-1166.

99. Little WC. Angiographic assessment of the culprit, coronary artery lesion before acute myocardial infarction. Am J Cardiol 1990, 66:44G-47G.

100. Kragel A, Gertz, SD, Roberts WC. Mophologic comparison of frequency and types of acute lesions in the major epicardial coronary arteries in unstable angina pectoris, sudden coronary death and acute myocardial infarction. J Am Coll Cardiol 1991, 18:801-808.

101. Fuster V, Frye RL, Kennedy MA, Connolly DC, Mankin HT. The role of collateral circulation in the various coronary syndromes. Circulation 1979, 59:1137-1144.

102. Habib GB, Heibig J, Forman SA, Brown BG, Roberts R, Terrin ML, Bolli R. Influence of coronary collateral vessels on myocardial infarct size in humans. Results of phase 1 thrombolysis in myocardial infarction (TIMI) trial. Circulation 1991, 83:739-746. 
103. Cohen M, Sherman W, Rentrop KP. Gorlin R. Determinants of collatera! filling observed during sudden controlled coronary artery occlusion in human, subjects. I Am Coll Cardiol 1989. $13: 29^{-7}-303$.

104. Fuster V. Stein B, Ambrose JA. Badimon L, Badimon J. Chesebro JH. Atherosclerotic plaque rupture and thrombosis: evolving concepts. Circulation 1990, 82(suppl II):1147.1159

105. Hackett D, Davies G. Chierchia S, Maseri A. Intermittent coronary occlusion in acute myocardiat infarction. Value of combined thrombolytic and vasodilator therapy. N Engl J Med 1987. 317:1055-1059

106. Bush L.R, Campbell WB, Tilton GD, Buja LM. Willerson JT. Effects of the selective thromboxane synthetase inhibitor, dazoxiben, on variations in cyclic blood flow in stenosed canine coronary arteries. Circulation 1984,69:1161-11701.

107. Ashton JIH, Benedict CR. Fitzgerald C, Raheja S, Taylor A, Campbell WB, Buja LM, Willerson JT. Serotonin as a mediator of cyclic flow variations in stenosed canine coronary arteries. Circulation [986, $73: 572-578$.

108. Ashton JH, Schmitz JM, Campbell WB, Ogletree MIL., Raheja S, Taylor AL., Fitzgerald C, Buja LM, Willerson IT: Inhibition of cyclic llow variations in stenosed canine coronary arteries by throm boxane $\mathrm{A}_{2}$ /prostaglandin. $\mathrm{H}_{2}$ receptor antagonists. Circ Res 1986, 59:568-578.

109. Golino P, Buia LM, Ashton JH, Kulkarni P, Taylor A, Willerson JT. Effect of thromboxane and serotonin receptor antagonists on intracoronary platelet deposition in dogs with experimentally stenosed coronary arteries. Circularion 1988, 78:701-711.

110. Griffith TM, Lewis MJ, Newby AC, Henderson AH. Endothelium-derived relaxing factor. I Am Coll Cardiol 1988, 12:797-806.

1 I. Christie D. Prevalence of stroke and its sequelae. Med J Aust 1981, ii:182-184.

112. Bonita R. Epidemiology of stroke. Lancet 1992, 339:342-344.

113. Shurtleff D. Some characteristics related to the incidence of cardiovascular disease and deatli: the Framingham Study 18 years follow-up. In: The Framingham Sudy, an epidemiological investigarion of cardiovascular disease. Kannel WB, Gordon T, eds. Publication (NIH) 74-599, Washington DC: US Department of Health, Education, and Welfare, 1974, Section 30.

114. Wolf PA, Kannel WB, Verter J. Current status of risk factors for stroke. Neurol Clin 1983 , $1: 317-343$.

115. Bonita R, Beaglehole R, North JDK. Event, incidence and case-fatality of cerebrovascular disease in Auckland, New Zealand. Am J Epidemiol 1984, 120:236-243.

1 I6. Marmot MG, Poulter NR. Primary prevention of stroke. Lancet 1992, 339:344-347.

117. Giaconi S, Levanti C, Fommei E, Innocenti F, Seghieri G, Palla L, Palombo C, Ghione S. Microalbuminuria and casual and ambulatory blood pressure monitoring in normotensives and in patients with borderline and mild essential hypertension. Am J Hypertens 1989, 2:259-261.

118. Kaplan NM. Clinical Hypertension. Baltimore, Md: Williams \& Wilkins, 5th ed. 1990.

119. Dworkin LD, Benstein JA. Impact of antihypertensive therapy on progressive kidney damage. Am J Hypertens 1989, 2:162S-172S. 
120. Lindeman RD, Tobin JD, Shock NW. Hypertension and the kidney. Nephron. 1987, 47 (suppl 1): $62-67$.

121. Pickering G. Hypertension Definitions, natural histories, and consequences. In: Itypertension: pathophysiology, diagnosis and management. Laragh JH and Bresner BM (eds). Raven Press New York, 1990, vol 1, pp3-20.

122. Messerli FH, Frohlich ED, Dreslinski GR, Starez DH, Aristimuno GG. Serum uric acid in essential hypertension: an indicator of renal vascular involvement. Ann Intern Med 1980, 93:817-821.

123. Eggers PW. Effect of transplantation on the Medicane End-Stage Renal Disease Program. N Engi I Med 1988, 3[8:223-229.

124. Weir MR, Hise Mk. Hypertensive renal damage. Cardiovasc Clin 1991, 21:115-132.

125. 1988 Joint National Committee: The !988 Report of the Joint Nationall Committee on Detection, Evaluation, and Treatment of High Blood Pressure. Arch intern Med 1988, 148:1023-1038.

126. Report of the British Ilypertension Society working party: Treating mild hypertension: Agreement for the large trals. Br Med 1 1989, 298:694-698.

127. Kaplan NM bongtem effectiveness of nonpharmacological treatment of byperiension. IJypertension 1994, [8(supph 1):1153-1160.

128. Frohlich ED, Messerli FH, Reisin ED, Dunn FG. The problem of obesity and hypertension. 1Hypertension 1983, 5: 11171-11178.

129. Kannel WB, Brand N, Skinner J, Dawber TR, MeNamara PM. Relation of adiposity to blood pressure and development of hypertension: the Franingham Study. Ann Intern Med 1967, $67: 48-59$

1.30. MacMahon SW, Blacket RB, MacDonald G]. Hall W. Obesity, alcohol consumption and blood. pressure in Australtan men and women. The National Heart Foundation of Austraka Risk Factor Prevalence Study. I Hyperiens 1984, 2:85-91.

131. Reisin E, Frohlich ED, Messerli FH, Dreslinski GR, Dunn FG, Jones MM, Batson HM. Cardiovascular changes after weight reduction in obesity hypertension. Ann Intern Med 1983, $98: 315-319$.

132. Reisin E, Abel R, Modan M, Silverberg DS, Eliahou HE, Modan B. Effect of weight loss without salt restriction on the reduction of blood pressure in overweight hypertensive patients. N Engl J Med $1978,298: 1-6$.

133. Landsberg L. Young JB. Fasting, feeding, and regulation of the sympathetic nervous system. N Engl ] Med 1978, 298: 1295-1301.

134. Kempner W. Treatment, of hypertensive vascular disease in rice diet. Am J Med 1948, 4:545.577.

135. Staessen J, Fagand R, Lijnen P, Amery A. Body weight, sodium intake and blood pressure. J Hypertens 1989, 7(suppl 1):\$19-\$23.

136. Morgan T, Nowson C. The role of sodium restriction in hypertension. Can J Physiol Pharmacol $1986,64: 786-796$

137. MacGregor GA, Markandu ND, Sagnella GA, Singer DRJ, Capuccio FP. Double blind study of three sodium intakes and long-term effects of sodium restriction in essential hypertension. Lancet $1989,2: 124+1247$. 
138. Weinberger MH, Cohen SJ, Miller JZ, Luft FC, Grim CE, Fineberg NS. Dietary sodium restriction as adjunctive treatment of hypertension. JAMA. 1988, 259:2561-2565.

139. Australian National. Health and Research Council Dietary Salt Study Management Committee, Fall in blood pressure with modest reduction in dietary salt intake in mild hypertension. Lancet 1989. 1:399-402.

140. Kaufman FL., Hughson RL., Schaman JP. Effect. of exercise on recovery blood pressure in normotensive and hypertensive subjects. Med Sci Sports Exerc 1987. 19:17-20.

141. Fagard R, Duprez D, Clement D. Amery A. Physical exercise th the management of hyperiension: a consensus statement of the World Hypertension League. 1 Hypertens 1991, 9:283-287.

142. Fagard R. Habitual physical activity, training, and blood pressure in nomo and hypertension. Int I Sports Med 1985, 6:57-67.

143. Seals DR. Hagberg JM. The effect of exercise training on human hypertension : a review. Med Sei Sports Exerc 1984, 16:207-215.

144. Van Bortel IMAB, Wijnen /AG, Kool MJF, van Baak MA, Struykes Boudier HA). Effect of exercise training on ambulatory blood pressure. J Hypertens 1992, 10(suppl 4):\$166

145. Gilders RM, Voner C, Dudley GA. Endurance training and blood pressure in normorensive and hypertensive adults. Med Sci Sports Exerc 1989, 21:629-636.

146. Van Hool R, Hespel P. Fagard R, Lijnen P, Staessen J. Amery A. Elfect of endurance training on blood pressure at rest, during exercise and during 24 hours in sedentary men. Am J Cardiol 1989, 63:945-949.

147. Tashiro E, Miura S, Koga M, Sasaguri M, Ideishi M, Ikeda M, Tanaka H, Shindo M, Arakawa K. The low workload exercise is betier than the high work load exercise for antihypertensive therapy. J Hypertens 1992, 10(suppl 4):\$87.

148. Blomquist CG, Saltin B. Cardiovascular adaptations to physical training. Ann Rev Physiol 1983 , 45:169-189.

149. Duncan J, Farr JE, Upton SJ, Hagan RD, Oglesby ME, Blair SN. The effects of aerobic exercise on plasma catecholamines and blood pressure in patients with mild essential hypertension. JAMA 1985, 254: 2609.2613

150. Meredith IT, Jennings GL, Esler MD, Dewar EM, Bruce AM, Fazio VA, Korner Pl. Time course of the antihypertensive and autonomic effects of regular endurance exercise in human subjects. J Hypertens $1990,8: 859-866$.

151. Jennings, G, Nelson L, Nestel P, Eslec M, Korner P. Burton D, Bazelmans J. The effects of changes in physical activity on major cardiovascular risk factors, hemodynamics, sympathetic function and glucose utilization in man: a controlted study of four levels of activity. Circulation 1986, $73: 30-40$.

152. Blair SN, Goodyear NN, Gibbons LW, Cooper KH. Physical fitness andincidence of hypertension in healthy normotensive men and women. JAMA 1984, 252:487-490.

153. Moore RD, L.cvine DM, Southard J. Entwisle G, Shapiro S. Alcohol consumption and blood pressure in the 1982 Maryland hypertension survey. Am J Hypertens 1990, 3:1-7.

154. Keil U, Chambless L, Filipiak B, Härtel U. Alcohol and blood pressure and its interaction with smoking and other behavioural variables: results from the MONICA Augsburg Survey 1984-1985. J Hypertens 1991, 9:491-498. 
155. Mizushima S, Nara Y, Mano M, Sawamura M, Horie R, Yamori Y. Alcohol consumption as a risk factor for high blood pressure from the Cardiovascular Diseases and Alimentary Comparison siedy f Cardievasc Pharmacol 1990, 16(suppl 8):535-537.

156. Patel C, Marmot MG, Terry DJ. Carruthers M, Hunt B, Patel M. Trial of relaxation in reducing coronary risk : four year follow up. Br Med I 1985, 290:1 103-1106.

157. var Montfrans GA, Karemakes jM, Wieling W, Dunning Al. Relaxation therapy and continuous ambulatory blood pressure in mild hypertension: a controlled study. Br Med J 1990 . $300 \cdot 1368-1371$

158. Stamler J, Rose G, Stamler R, Elliot P, Dyer A, Marmot M. INTERSALT study findings: Public health and medical care implications. Hypertension 1989, 14:570-577.

159. Prichard BNC, Owens CWI. Drug treatment of hypertension. In: Hypertension. Genest J, Kuchel O, Jamet P, Cantin M (eds). McGraw-Hill, New York, 1983, pp 1171.1210.

160. Hypertension In: Betableckers in chinical practice. Cruickshank: IM, Prichard BNC (eds). Churchili Livingstone, Edinburgh, London, Melboume and New York, 1987, pp275-433.

16I. Jouston MC. New insights and new approaches for the treatment of essential hypertension: selection of thempy based on coronary heart disease risk factor analysis, hemodynamic profiles, quality of life, and subseis of hyperiension. Am Heart J 1989, 117:911.944.

162. Finnerty FA Jr, Maxwell MH, Lunn J, Moser M. Long-term effects of furosemide and hydrochlorothiajde in patienis with essential hypertension. A two-year comparison of efficacy and safety, Angiology 1977, 28:125-133.

163. Van Bortel I,MAB, Kool MJE, Breed JGS, Lustermans FAT, Mooi JMV, De Cree J. In contrast to aienolal, nebivolol does not increase systemic vascular resistance. Eur Heart J 1992, 13(Abstract stippl), 21 .

164. L.hing B. Vascular selectivity of felodipine. Drugs 1985, 29(suppl 2): $46-58$.

165. Brunner IIR, Waeber B, Nussberger J. What wouk we like to know about the antihypertensive mechanisrns of angiotensin converting enzyme inhibition. J Hypertens 1988, 6(suppl 3):S1-55.

166. Dzau VI, Safar ME. Large conduit arteries in hypertension: role for the vascular renin-angiotensin system. Girculation 1988, 77:947-954.

167. Weinberger MH. Optimizing cardiovascular risk reduction during antihypertensive therapy. Hypertension 1990, 16:201-211.

168. Khoury AF, Kaplan NM. Alpha-blocker therapy of hypertension. An unfulfilled promise. JAMA 1991, 266:394-398.

169. O'Rourke M: Arterial stiftness, systolic pressure, and logical treatment of arterial hypertension. Hypertension 1990, 15:339-347.

170. Duchier J, Lannascoll F, Safar M. Antihypertensive effect of sustained-release isosorbide dinitrate for isolated systolic systemic hypertension in the elderly. Am I Cardiol 1987, 60:99-102.

171. Cruickshank JM. Coronary flow reserve and the J shaped relation between diastolic blood pressure and myocardial infarction. Br Med J 1988, 297:1227-1230.

172. Lijnen P, Fagard R, Staessen J, Vanhees L, Amery A. Mechanism of hypotensive action of ketanserin in men. Eur Heart J 1987, 8:308-312. 
173. Noble MIM, Drake-Holland AJ. The involvement of serotonin in the formation of thrombi at critical coronary arterial stenoses in humans. Coronary Artery disease 1990, 1:675-679.

174. Laragh. JH. Strategies in choosing therapy for hypertension. In: New therapeutic strategies in hypertension. Kaplan NM, Brenner BM, Laragh IH (eds), Raven Press, New York, 1989. Ppl 1 1-169.

175. Brunner HR. Ménard J. Waeber B. Burner M, Biollaz I, Nussberger J. Bellet M. Treating the individual hypertensive patient: considerations on dose, sequential monotherapy and drug combinations j Hypertens 1990, 8:3-11.

176. Harington M. Kincaid-Smith P. McMichael J. Results of treatment in malignant hypertension. A seven-year experience in 94 cases Br Med I 195911.969979.

177. Collins R. Peto R, MacMahon S, Hebert P. Fiebach NH, Eberlein KA, Godwin J. Qizilbash N, Tayjor JO, Hennekens CH. Blood pressure, stroke, and coronary heart discase. Part 2, short-term reductions in blood pressure: overview of randomised drug trials in their epidemiological context. lancet 1990, 335:827-838.

178. Pardell H, Armario P. Hernandez R. Progress in the 1980 s and new directions in the 1990 with hypertension management. From stepped-care approach to individualized programme in hypertension treatment and control. Drugs 1992, 43:1.5

179. Haynes RB, Taylor DW, Sacket DL, Gibson ES, Bemholz CD, Mukherjec J. Can simple clinical measurements cietect patient noncompliance? Hypertension 1980, 2:757-764.

180. National Heart, Lung, and Blocd Institute Working Group. Management of patient compliance in the treatment of hypertension. Hypenension 1982, 4:415-423.

181. Weber MA. Factors affecting compliance in the management of hypertension. Prim Cardiol 1985. $2: 35-39$

182. Eisen SA, Miller DK, Woodward RS, Spitznagel E, Prezybeck TR. The effect of preseribed daily dose frequency on patient medication compliance. Arch Intern Med 1990, 150:1881-1884.

183. Inui TS, Carter WB, Pecoraro RE. Screening for noncompliance among patients with hypertension: Is self-report the best available measure? Med Care 1981, 19:1061-1064.

184. Widmer RB, Cadoret RI, Troughton E. Compliance characteristics of 291 hypertensive patients from a rural midwest area. J Fam Pract 1983, 17:619-625.

185. Haynes RB, Gibson ES, Taylor DW, Bernholz CD, Sackett DL. Process vs outcome in hypertension: a positive result. Circulation $1982,65: 28-33$.

186. Williams GH. Quality of life and its impact on hypertensive patients. Am J Med 1987, 82:98-105.

187. Winickoff RN, Murphy PK. The persistent problem of poor blood pressure control. Arch Intern Med 1987, 147:1393-1396.

188. Messerli FH, Nunez BD, Nunez MM, Garavaglia GE, Schmieder RE, Ventura HO. Hypertension and sudien death. Arch Iritern Med 1989, 149:1263-1267.

189. Messerli FH. Left ventricular hypertrophy, arterial hypertension and sudden death. J Hypertens 1990, 8(suppl 7):S181-\$185.

190. Koren MI, Ulin RJ. Laragh JH, Devereux RB. Reduction of LVM during treatment of essential hypertension is associated with improved prognosis. Am I Hypertens 1991, 4: 1A-2A. 
191. Dahlof B, Pennert K, Hansson 1. Reversal of left ventricular hypertrophy in hyperiensive patients. A metaanalysis of 109 treatment studies. Am J Hypertens 1992, 5:95-110.

192. Cohn JN, Johnson G, Ziesche SM, Cobb F, Francis G, Tristani F, Smithi R, Dunkman. WB, Loeb $H$, Wong $M$, Bhat $G$, Goldman S, Fletcher RD, Doherty J, Hughes CV, Carson P, Cintron G, Shabetai R, Hakenson C. A comparison of enalapril with hydralazine-isosorbide dinitrate in the ireatment of chronic congestive heart failure. N Engl i Med 1991, 325:305-310.

193. Cohn JN, Archibald DG, Ziesche S, Franciusa JA, Harston WE Tristani FE, Dunkman WB, Jacobs W, Francis GS, For KH, Goldman S, Cobb FR, Shah PM, Saunders R, Fletcher RD, Loeb HS, Hughes VC, Baker B. Effect of vasodilator therapy on mortality in chronic congestive heart failure: results of a Veterans Administration Cooperative Study. N Engl I Med 1986, $314: 1547-1552$.

194. Pfeffer MA, Braunwald E, Moyé LA, Basta L. Brown E], Cuddy TE. Davis BR, Geitman EM, Goldman S, Flaket GC, Klein M, Lamas GA, Packer M, Rouleau J. Rouleau Jl, Rutherford J. Wertheimer JH, Hawkins CM, The SAVE Investigators. Effect of captopril on mortality and morbidity in patients with left ventricular dysfunction after myocardial infarction. Resules of the Survival and Ventricular Enlargement Iirial. N Engl J Med 1992, 327:669-677.

195. Swedberg K. Held P, Kiekshus J, Rasmussen K, Ryden L, Wedel H, The CONSENSUS II Study Group. Flfects of the early administration of enalapril on monality in patients with acute myocardial infarction. N Eingl J Med 1992, 327:678-684.

196. Ambrosioni E, Borghi C, Magnani B, The SMILE Pilot Study Working Party. Early treatment of acute myocardial infarction with angiotensin-converting enzyme inhibition: safety considerafions. Am / Gardiol 1991, 68:101D-1101).

197. Schoemaker RG, Debets JJ, Struyker Boudier HA, Smits JF. Delayed but not immediate captopril therapy improves cardiac function in conscious rats, following myocardial infarction. J Mol Cell Carcliol 1991, 23:187.197.

198. Stary HC. Evolution and progression of atherosclerotic Iesions in coronary arteries of children and young adults. Atherosterosis 1989, 99(supp! 1):119-132.

199. Castellot J, Cochran D. Karnovsky M. Effect of heparin on vascular smooth muscle cell. I. Cell metabolism I Cell Physiol 1985, 124:21-38.

200. Cochran D, Castellot 1. Karnovsky M. Effect of heparin on vasculat smooth muscle cell. II. Specific protein synthesis. J Cell Physiol 1985, 124:39-46.

201. Fox PL, Dicorleto PE. Fish oil inhibits endothelial cell production of platelet derived growth factor-like protein. Science 1988, 241:453-456.

202. Huang SS. Ihang IS. Rapid tumovex of platelet-derived growth factor receptor in sis-transformed cells and reversal by suramin. I Biol Chem 1988, 263:12608-12612

203. Zanchetti A. The antiatherogenic effects of antihypertensive drugs: experimental and clinical evidence. Clin Exp Hypertens 1992, A14(1\&2), 307-331.

204. Steinberg D. Modified forms of LDL and their pathophysiologic significance. Presented at the 8th International Symposium on Atherosclerosis. Rome: ClC Edizioni International, 1988: 1-7.

205. Carew TE. Role of biological modified LDL in atherosclerosis, Am J Cardiol 1989, 64:18G-22G. 
206. Carew TE. Schwenke DC, Steinberg D. Antiatherogenic effect of probucol unrelated to its hypocholesterolemic effect: evidence that antioxidant in wo can selectively inhibit $1 \mathrm{DL}$. degradation in macrophage risk tatty streaks and slow the progression of atherosclerosis in the Watanabe heritable hyperlipidemic rabbit. Proc Natl Acad Sci l\$A 1987, 84, $7725,7729$.

207. Lichtlen PR. Hugenholtz PG, Rafflenbeul W, Hechker H, Jost S, Decker JW. Retardation of angiographic progression of coronary heart discase by nifedipine 1ancet $1990,3,35: 1109-1113$.

208. Kensy PD. Antiperoxidative actions of calcium antagonists and atherogenesis, / Cardiovasc Pharmacol 1\%)1, 18(suppl 1):S6-\$10.

209. Büher FR. The case for calcium antagonists as first-line treatment of hypertension. J Hypertens 1992, 10(suppl 3):\$17.\$20.

210. Brown G, Albers J], Fisher LD, Schacfer SM, Lin J-T, Kaplan C, Thao X-Q, Bisson BD, Fitzpatrick V7. Dodge HT. Regression of coronary artery disease as a result of intensive lipid-lowering therapy in men with high levels of apolipoprotein B N Engl ] Med 1990, 323:1289-1298.

211. Buchwald H, Varco RI, Mats JP, Long JM, Fith 1.l, Campbell GS, Pearce Ml, Yellin AE, Edmision WA, Smink RD, Sawin HS, Campos CT, Jansen B], Tuna N, Kamegis IN, Sanmarco ME, Amplatz K, Castaneda-Zuniga WR, Hunter DW, Bisset JK, Weber FJ, Stevenson IW, Leon AS. Chalmers TC. POSCH Group. Effect of partial leal bypass surgery on mortality and morbidity from coronary hean disease in patients with hypercholesterolemia. N Engl I Med 1990, $323: 946-955$.

212. Fuster V, Badimon L, Badimon J, Chesebro JH. The pathogenesis of coronary artery disease and the actie coronary syndromes. (second of two parts). N Engl I Med 1992, 326:310-318.

213. Pedersen T. A multicenter trial on timolol in secondary prevention after myocardial infarction. Acta Med Scand 1983, 674(suppl):1-129.

214. Olsson G, Rehnqvist N. Reduction of non-fatal rcinfarctions in patients with a history of hypertension by chronic postinfarction treatment with metoprolol. Acta Med Scand 1986, 220:33-38.

215. Wikstrand J. Reducing the risk for coromary heart disease and stroke in hypenensives - comments on mechanisms for coronary protection and quality of life. J Clin Pham Ther 1992, 17:9-29.

216. Fitggerald JD. By what means might beta blockers prolong life after acute myocardial infarction? Eur Hear J 1987, 8:945-951.

217. The Danish Study Group On Verapamil In Myocardial Infarction. Effect of verapamil on mortality and major events after acute myocardial infarction (The Danish Verapamil Infarction Trial II: DAVTT II). Am J Cardiol 1990, 66:779-785.

218. The Mulicenter Diltiazem Pastinfarction Trial Research Group. The effect of diltiazem on martality and reinfarction after myocardial infarction. N Engl J Med 1988, 319:385-392.

219. Chesebro JH, Webster MWI. Smith HC, Frye RL, Holmes DR, Recder GS, Bresnahan DR, Nishimura RA. Clements IP. Bradsley WT, Grill DE, Fuster V. Antiplatelet therapy in coronary disease progression: reduced infarction and new lesion formation. Circulation 1989, 80(suppl II) : 11.266 abstract.

220. Antiplatelet Trialists Collaboration. Secondary prevention of vascular disease by prolonged antiplatelet trearment. Br Med J 1988, 296:320-331

221. ISIS-z (Second International Study of infarci Survival) Collaborative Group. Randomised trial of intravenous streptokinase, oral aspirin, both or neither among 17187 cases of suspected acute myocardial infarction: ISIS-2. Jancet 1988, 2:349-360. 
222. Hennekens CH, Buring JE. Sandercock P. Collins R. Peto R. Aspirin and other antiplatelet agents in the secondary and primary prevention of cardiovascular disease. Circulation 1989. $80: 749-756$.

223. Stein B, Fuster V. Clinical pharmacology of platelet inhibitors. In: Fuster V. Verstraete M, eds. Thrombosis in cardiovascular disorders. Philadelphia: W.B. Saunders, 1992:99-120.

224. Smith P. Antithrombotic therapy in the chronic phase of myocardial infarcion. In: Fuster V, Verstracte M, eds. Thrombosis in cardiovascular disorders. Philadelphia: W.B. Saunders, $1992: 343-362$.

225. Kiowski W', Luscher TF, linder I., Buhler FR. Endothelin-1 induced vasoconstriction in humans: reversal by calcium channel blockade but not by nitrovasodilators or endothelium-derived relaxing factor. Circulation 1991, 83:369-475.

226. van Gilst WH, Kingma III, CATS Investigator Group, Early intervention with angiosensinconverting enzyme inhibitors during thrombolytic therapy in acute myocarcial infarction: mionale and design of captopril and thrombolysis study. Am J Cardiol 1991, 68:111D-115D.

227. Perondi R, Sano A, Tio RA, Pomidossi G, Gregorini L. Alessio P, Morganti A, Zancheti A, Mancia G. ACE inhibinon attenuates sympathetic coronary vasoconstriction in patients with coronary artery disease. Circulation 1992, 85:2004-2013.

228. Beme C. Pollare T, Lihell H. Effects of antihypertensive treatment on insulin sensitivity with special reference to ACE inhibition. Diabetes Care 1991, 14(suppl 4):39-47.

229. I. wheli H. Metabolic effects of antihypertensive drugs interacting with the sympathetic nervous system. Am lleart j 1992, 13(suppl A) :53-57.

230. Santuce A, Fern C. Insulin resistance and essential hypertension: pathophysiologic and therapeutic implications. J Hypentens 1992, 10(suppl 2):59-815.

231, Schmieder $R$, Friedrich $G$, Neus H, Rudel H, von Etf AW. The influence of beta-blockers on cardovascular reactivity and type 'A' behaviour pattern in hypertensives. Psychosom Med 1983. $45: 417-423$.

232. Kranz DS, Durel LA, Davia JE, Shafer RT, Arabian JM, Dembroski TM, MacDougall JM. Propranolol medication among coronary patients: relationship to type A behaviour and cardiovasculat response. J lluman Stress 1982, 8:4-12.

233. Fricdman M, Thoresen CE, Gill $\mathrm{l}$. Alteration of type 'A' behaviour and its effect on cardiac recurrences in posi myocardial infarction patients: Summary results of the recurrent coronary preventhon project. Am Heart I 1986, 112:653-665.

234. Martin M, Hulley S. Browner W; Kuller L, Wentworth D. Serum cholesterol, blood pressure, and mortality: implications from a cohor of 361,662 men. Lancet 1986, 2:933-936.

235. Davis CE, Rifkind BM, Brenner H, Gordon Dj. A single cholesterol measurement underestimates the risk of cononary heart disease. JAMA. 1990, 264:3044-3046.

236. The Lipid Research Cliniss Coronary Primary Prevention Trial results. I. Reduction in incidence of coronary heart disease. JAMA 1984, 251:351-364.

237. Canner PL. Berge KG. Wenger NK, Stamler J, Friedman L, Prineas RJ. Friedewald W. Fifteen year mortality in Coronary Drug Project patients: long-term benefit with niacin. J Am Coll Cardiol $1980,8: 1245-1255$. 
238. Frick MH, Elo O, Haapa K, Heinonen OP, Heinsalmi P, Helo P, Huttunen IK, Kaitaniemi P. Koskinen P, Manninen V, Mäenpaa H, Malkonen M, Manttari M, Norola S, Pasternack A, Pikkarainen J. Romo M, Sjoblom T, Nikkilä EA. Helsinki Heart Study: Primary-prevention trial with gemfibrozil in middle-aged men with dyslipidemia. N Engl J Med 1987. 317:1237-1245.

239. Rossouw JE, Lewis B, Rifkind BM. The value of lowering cholesterol after myocardial infarction. N Engl I Med 1990, 323:1112-1119.

240. Jacobs D, Blackburn H, Higgins M, Reed D, Iso H, McMillan G, Neaton J, Nelson J, Potter J, Rifkind B, Rossouw J. Shekelle R, Yusuf S. Report of the conference on low blood cholesterol: mortality associations. Circulation 1992, 86:1046-1060.

241. Ueda K. Hasua Y, Kiyohara Y, Wada I, Kawano H, Kato 1, Fujit 1, Yanai T, Omac T, Fujishima M. Intracerebral hemorrhage in a Japanese community. Hisayama: incidence, changing pattern during long-term follow-up, and related factors. Stroke 1988, 19:48-52

242. Ueshima H, lida M, Simamoto T, Komishi M, Komachi Y. Multivariate analysis of risk factors for Stroke: eight-year follow-up study of farming villages in Akita, Japan. Prev Med 1980, 9;722-740.

243. Muldoon MF, Manuck SB, Mathews KM. Lowering cholesterol concentrations and mortality: a quantitative review of primary prevention trials. Br Med J 1990, $304: 30 \%-314$.

244. Committee of Principal Investigators. A co-operative trial in the primary prevention of ischaemic heart disease using clofibrate. Br Heart J 1978, 40:1069-1 118.

245. Goldman L, Gordon DJ, Rifkind B. Hulley S, Detsky AS, Goodman DS, Kinosian B, Weinstein MC. Cost and health implications of cholesterol lowering. Circulation 1992, 85: 1960-1968.

246. Cruickshank JM, Smith JC. The beta-receptor, atheroma and cardiovascular damage. Pharmacol Ther $1989,42: 385-404$.

247. Linden T, Camejo G, Willund O, Warnold I, Olofsson S-O, Bondjers G. Effect of short term betablockade on serum lipid levels and on interaction of LDL with human arterial proteoglycins. J Clin Pharmacol 1990, 30(suppl):S124-\$131.

248. Amery A, Birkenhäger W, Brixko P, Bulpith C, Clement D, Denyttere M, De Schaepdryver A, Dollery L, Fagard R, Forette F, Forte J, Hamdy R, Henry JF, Joossens JV, Leonetti G, I.undJohansen P, O'Malley K, Petrie J, Strasser T, Tuomilehto J, Williams B. Mortality and morbidity results from the European Working Party on High Blood Pressure in the Elderly trial. Lancet 1985. i: $1349-1354$.

249. Warlow C. Secondary prevention of stroke. Lancet 1992, 339:724-727.

250. Hankey GI, Slattery JM, Warlow CP. The prognosis of hospital-referred transient ischaemic auacks. J Neurol Neurosurg Psychiatry 1991, 54:793-802.

251. Sandercock P, Willems. H. Medical treatment of ischaemic stroke. Lancet 1992, 339:537-539.

252. Whelton PK, Klag MJ. Hypertension as a risk factor for renal disease: review of clinical and epiclemiological evidence. Hypertension 1989, 13(suppl I):119-27.

253. O'Rourke MF. Basic concepts for the understanding of large arteries in hyperiension. J Cardiovase Pharmacol 1985, 7(suppl 2):S14-S21.

254. Safar ME, Simon AC, Levenson JA. Structural changes of large arteries in sustained essential hypertension. Hypertension 1984, 6(suppl III): 111.117. 
255. Reneman RS, van Merode T. Hick P. Hoeks APG. Flow velocity patterns in and distensibility of the carotid artery bulb in subjects of various ages. Circulation 1985, 71:500-509.

256. Kawasaki T, Sasayama S, Yagi S, Asakawa T, Hirai T. Non-invasive assessment of the age relared changes in stiffness of maịor branches of human arreries. Cardiovasc Res 1987, 21:678-687.

257. Levenson jA, Safar ME, Simon AC. Boulies I/A, Gricher I. Systemic and arterial hemodynamic: effects of nifedipine $(20 \mathrm{mg})$ in mild-to-moderate hypertension. Hypertension 1983, 5(suppl V): $\mathrm{V} 57 . \mathrm{V} 60$.

258. Van Merode T, Van iłortel I., Smeets F, Bohm R. Mooil J, Rahn KH, Reneman RS. The effect of verapamil on carotid antery distensibility and cross-sectional compliance in hypertensive patients. J Cardiovase Pharmacol 1990, 15:109-113.

259. Safar M, Bons J, Georges D, Cournot A, Duchier J. Calcium blocker lacidipine and carotid arteriolar vasodilation in healthy volunteers. Clin Pharmacol Ther 1989, 46:94-98.

260. Levenson J, Simon AC. Bouthier J, Marek BC; Safar ME. The cffect of acute and chronic nicardipine therapy on forearm arterial haemexlynamics in essential hypertension. Br f Clin Pharmac. $1985,20: 107 \mathrm{~S}-113 \mathrm{~S}$.

261. Bouthier J1, Safar ME, Benctos A, Simon AC, Levenson JA, Hugues CM Matmodynamic effects of vasodilating drugs on the common carotid and brachial circulations of patients with essential hypertension Br J C.lin Pharmac 1986, 21:137-142.

262. Simon AC, Levenson J. Bouthier JD, Safar ME. Effects of chronic administration of enalapril and propranolol on the large arteries in essental hypertension. J Cardiovasc Pharmacol 1985 , $7: 856-861$.

263. Asmar RG, Pannier B, Santoni JP, Laturent S, London GM, Levy BI, Safar ME. Reversion of cardiac hypertrophy and reduced arterial compliance after converting encyme inhibition in essential hypertension. Girculation 1988, 78:941.950.

264. Marek 13L, Bouthier JA, Simon AC., Levenson JA, Safar ME. Comparative effects of propranolol and pindolol on small and large ancries and veins of the forearm circulation in hypertensive man. J Cardiovasc Pharmacol 1986, 8(suppl 4):\$61-\$66.

265. Van Bortel LMAB, Van Merode T, Smeets FAM, Reneman RS, Mooij JMV, Struyker Boudier HAJ. Nebivolol improves the vessel wall properties of the common carotid ariery. Drug lnvest 1991 , $3($ suppl 1):61-63

266. Kelly RP, Daley IE, Avolio AP, O'Rourke MF, Arterial dilation and reduced wave reflection: Beneft of dilevalol over atenolol in essential hypertension. Hypertension 1989, 14:14-21.

267. Pithois-Merli I. Levenson J. Filitti V. Simon A. Comparative long-term vasoactive effects of atenolol and cartcolol on the properties of the small and large arteries of the upper extremities in humin essential hypertension. Clin Pharmacol Ther 1989, 46:686-692.

268. I.evenson 1, Le Quang Sang KH, Devynck MA, Gitel R, Simon A. The role of antihypertensive drugs in counteracting adverse influence on large arteries. Am Heart y 1987, $114($ suppl 4) part II: $992-997$.

269. Simon AC, Levenson JA, Levy BY, Bouthier JE, Peronneau PP, Safar ME. Effect of nitroglycerin on peripheral large arteries in hypertension. Br J Clin Pharmac 1982, 14:241-246. 
270. De Luca N. Rosiello G. Crispino M. Volpe M, Galetti P. Buzetti G. Trimarco B. Effects of chronic antihypertensive treatment with ketanserin versus metoprolol on blood pressure and targe arteries compliance in humans: a cross-over double-blind study. I Clin Pharmacol 1988 , $28: 332-338$.

271. Asmar RG. Kerihuel JG, Girerd Xj, Safar ME. Effect of hisoprolol on blood pressure and anterial hemodynamics in systemic trypertension. Am J Cardiol 1991, 86:61-64.

272. Laurene S, Lacolley PM, Cuche JI., Safar ME. Influence of diuretics on brachial artery diameter and distensibility in hypertensive patients. Fundam Clin Pharmacol 1990, 4.685-693.

273. Bouthier JA, Safar ME, Curien ND, London GM, Levenson JA, Simon AC. Effect of cadralazine on brachial artesy hemodynamics and forearm venous tone in essential hypertension. Clin Pharmaicol Ther $1986,39: 82-88$

274. Achimastos A, Girerd X. Simon A, Pithois-Merli I, Levenson I. The efficacy of transdermat formulation of clonidine in mild to moderate hypertension and ats effects on the arterial and venous vasculature of the forearm. Eur J Clin Pharmacol 1987, 33:111-114.

275. Yaginuma T, Avolio A, ORourke MF, Nicholls WW, Morgan J. Roy P, Baron D, Branson y. Feruetey M. Effects of glyeeryl uninitrate on peripheral arteries alters teft ventricular hydraulic load in man. Cardiovasc Res 1986, 20:15,3-160.

276. Fitchett D, Sinkus G, Beatdry J, Marpole D. Reflected pressure waves in the ascending aora: Effect of glyceryl trinitrate. Cardiovasc Res 1988, 22:494-500.

277. Gessner FB. Ifernodynamic theories of atherogenesis. Circ Res 1973, 33:259-266.

278. Joris I, Majno G. Inflammatory components of atherosclerosis. In: Weissman G, Samuelson H, Paoletti R (eds). Advances in inflammation research. New York, Raven Press, 1979:71-85.

279. Schaffiner T. Taylor K, Bartucci E], Fischer-Dzcgat K, Beeson III, Glagov S, Wissler RW. Arterial foam cels exhibit distinctive immunomorphologic and histochemical features of macrophages. Am J Pathol 1980, 100:57-80.

280. Uematsu M, Kitbatake A, Tanouchi J, Doi Y, Masuyama T, Fujii K, Yoshida Y, Ito Hiroshi. Jshihara $\mathrm{K}$, Hori M, Inoue M, Kamada $\mathrm{T}$. Reduction of endothelial microfilament bundles in low-shear region of the canine aorta: association with intimal plaque formation in hypercholesterolemia. Atherosclerosis and Thrombosis 1991, 11:107-115.

281. Glagov S, Oroa A.K. Significance of the relatively low incinlence of atherosclerosis in the pulmonary, renal and mesenteric arteries. Ann NY Acad Sci 1968, 149:940.955.

282. Glagow S, Rowley DA, Kohut R. Adherosclerosis of human aorta and its coronary and renal arteries. Arch Pathol Lab Med 1961, 72:558-571.

283. Klocke FJ, Mates RE, Canry JM, Ellis AK. Coronary pressure-flow relationships: controversial issues and probable implications. Circ Res 1985, 56:310-323.

284. Perski A, Olsson G. Landou C, de Faire U, Theorell T, Hamsten A. Minimum heart rate and coronary atherosclerosis: independent relations to global severity and rate of progression of angiographic lesions in men with myocardial infarction at a young age. Am Heart J 1992, 123:609-616.

285. Falk. E. Coronary thrombosis: Pathogenesis and clinicall manifestations. Am J Cardiol 1991, 68: 28B-35B. 
286. Bassenge F. Flow-dependent regulation of coronary vasomotor tone. Eur Heart / 1989, 10(suppl F), $22-27$.

287. Simon AC, Pithois-Merli I, Levenson J. Physiopharmacological approach to mechanical factors of hypertension in the atherosclerotic process.J Hum Hypert 1991, 5(suppl 1), 15-21.

288. Fleckenstein A. Vasodilator actions of calcium antagonists on peripheral resistance vessels in hypertension. In: Calcium antagonism in heart and smooth muscke (ed: Fleckenstein A). Wiley and Sons, New York, 1983, pp267.272.

289. Arndt JO, Klauske J, Mersch F: The diameter of the intact carotid artery in man and its change with pulse pressure. Pligers Arch 1968, 301:230-240.

290. Mozersky DJ. Sumner DS, Hokanson DE, Strandness DE. Transcutancous measurement of the elastic properties of the human femoral artery. Circulation 1972, 46:948-955

291. Hocks APG, Ruissen C., Hick P. Reneman RS. Transcutaneous detection of retative changes in artery diameter, Uhtrasound Med Biol 1985, 11:51-59.

292. Reneman RS, Van Merode T, Hick PIJ. Hocks APG. Cardiovascular applications of multigate pulsed Doppler systems. Ultrasound Med Biol 1986, 12:357-370.

293. Hocks APti, Reneman RS, Peronneau PA. A multigate pulsed Doppler system with serial data processing. IEEE Trans Sonics Utrason 1981, 28:242-247.

294. Hoeks APG. Pecters HPM, Ruissen CI. Renenan RS. A novel frequency estimator for sampled Doppler signats, IEEE Trans Biomed Eng 1984, 31:212-220.

295. Reneman RS, Van Merode T, Hick PJ]. Muytjens AMM, Hoeks APG, Age-related changes in carotid anery wall properties in man. Uhtrasound Med Biol 1986, 12:465-471.

296. Pessenhofer il. Single cuff comparison of two methods for indirect measurenent of arterial blood pressure : standlard auscultatory method versus automatic oscillometric method. Basic Res Cardiol 1986, $81: 101-109$.

297. Koch GG. The use of non-parametric methods in the statistical analysis of the two-period changeover design. Biometries 1972, 28:577.584.

298. Van Merode T, Hick PII. Hoeks APG, Rahn KH, Reneman RS. Carotid antery wall properties in normotensive and borderline hypertensive subjects of vanous ages. Ultrasound Med Biol 1988, $1 \cdot 4: 563-569$.

299. Laurent S, Arcaro G, Benetos A, Lafleche A. Hocks A, Safar M. Mechanism of nitrate-induced improvement on arterial compliance depends on vascular territory. J Cardiovasc Pharmacol 1992, $19: 641-649$

300. Robertson JIS. The large studies in hypertension: what have they shown? Be J Clin Pharmacol $1987.124($ suppl) $: 3.14$.

301. Simon ACH, Laurent A, Levenson JA, Bouthier J-E, Safar ME. Estimation of forearm arterial compliance in normal and hypertensive men. Cardiovase res 1983, 17:331-338.

302. Van de Water A, Janssens W, Van Nueten J, Xhonneux R, De Cree J, Verhaegen H, Reneman RS, Janssen PA. Pharmacological and hemodynamic profile of nebivolol, a biochemically novel, potent, and selective B1-adrenergic antagonist. J Cardiovasc Pharmacol. 1988, 11: 552-563. 
303. Schnaper H, Jackson D. Sit SP. Nebivolol: a new generation of $\beta$-blockers in hypertension. Am I Hypertension [991, 4:23A.

304. Sieben G, Van Nueten I., Symoens. J. Nebivolol in hypertension Drug Imvest 1991, 3(suppl 1):190-192.

305. Wenger NK, Furberg CD. Cardiovascular disorders. In: Quality of life assessments in clinical urials Spilker B (Ed), Raven Press New York, 1990: pp335-345.

306. Zachariah PK Quality of life with antihypertensive medication. J Hypertens 1987, 5(suppl 4) :\$105.\$110.

307. Wassertheil-Smoller S, Blaufox D, Oberman A, Davis B, Swencionis C, O'Connell Knerr $M$, Hawkins MC, Langford HG. Effect of antihypertensives on sexual function and quality of the: The TAIM study. Ann intern Med 1991, 114:613-620.

308. Deyo RA. The quality of life, research, and care. Ann intern Med 1991, 114:695-696.

309. Jaeschke R, Guyatt GH. How to develop and validate a new quality of life instrument. In: Quality of life assessments in clinical trials. Spilker B (Ed), Raven Press New York, 1990:47-57.

310. Visser Afh. De betekenis van de VOEG : enkele gegevens over de begripsvaliditeit. Gezondheid \& Samenkving 1983, 4:177-188.

3.1 I. Joosten J, Drop MJ. De berrouwbaarheid en vergelijkbaarheid van drie versies van de VOEG. Gezondheid \& Samenleving 1987, 8:251-265.

312. van Reek ], Diederiks ], Philipsen H, van Zutphen W, Seelen T. Subjective complaints and blood pressure. J Psychosom Res 1982, 26:155-165.

313. Herrick AL, Waller PC, Berkin KE, Pringle SO, Callender JS, Robertson MP, Andlay JG, Murray GD, Reid Jl, Lorimer AR, Weir RJ. Carmichael HA, Robertson JIS, Ball SG, McInnes GT. Comparison of enalapril and atenolol in mild to moderate hypertension. Am J Med 1989, 86:421-426.

314. Gabo H, Holst J, Christensen NJ, Hilsted J. Glucagon and plasma catecholamines during betareceptor blockade in exercising man. J Appl Physiol 1976, 40:855-863.

315. Lundborg P, Ästrom H, Bengtsson C, Fellenius E, von Schenck H, Svensson I, Smith U. Effect of B-adrenocepror blockade on exercise performance and metabolism. Clin Sci 1981, 61:299-305.

316. Kullner $T$, Kindermann $W$, Singer $M$. Effects on physical performance of intrinsic sympathomimetic activity (ISA) during selective B1-blockadc. Eur J Appl Physiol 1987, 56:292-298.

317. Van Baak MA, Bohm ROB, Arends BG, Van Hooff MEJ, Rahn KH. Long-term antihypertensive therapy with beta-blockers : submaximal exercise capaciry and metabolic effects during exercise. Int J Sports Med 1987, 8:342-374.

318. Van Baak MA, Bóhm ROB, Petri J, Rahn KH. Metabolic effects of verapamil and propranolol during submaximal endurance exercise in patients with essential hypertension. Int J Sports Med $1987,8: 270-274$.

319. Cléroux I. Van Nguyen P, Taylor AW, Leenen FHH. Effects of $\beta_{1}$ vs $\beta_{1}+\beta_{2}$ blockade on exercise endurance and on muscle metabolism in humans. J Appl Physiol 1989, 66:548-554.

320. Vanhees L, Fagard R, Lijnen P, Amery A. Effect of antihypertensive medication on endurance exercise capacity in hypertensive sportsmen. J Hypertens 1991, 9:1063-1068. 
321. Kaplan CA, Camachoi 'T. Perceived. Health and Mortality: a ten year follow up of the human population cohort. Am j Epidemiol 1983, 117:292-304.

322. Bazet IIC. An analysis of time-felations of electrocardiograms. Heart 1920, 7:353.

323. Medical Research Council Working Party. MRC, tral of treatment of mild hypertension: principal results. Br Med J 1985, 291:97-104

32- Capone P. Mayol R. Placebo-controlled double-blind multicenter study of celiprolol in the treatment of mild and moderate hypertension. I Cardiovasc Pharmacol 1986, 8(suppl 4):5119-5121.

325. Bühler FR, Berglund G, Anderson OK, Brunner HR. Scherrer C, van Brummelen P. Dister A, Philipp Th, Fogari R, Mimran A, Foureade J, dal Palu C. Prichard BNC, Backhouse Cl, Reid Jl, Filiot H. Yanchett A. Doubleblind companison of the cardioselective B-blockers bisoprolol and atenolol in hypertension: the bisoprolol international multicenter study (BIMS). J Cardiovase Pharmacol i986, 8(suppl 11):S122-\$127.

326. Weinberger MIt. The retationhip of sodium balance and conconitant diuretic therapy to blood pressure response with calcium entry blockers. Am J Med 1991, 17:155-20S.

327. Seides S7, Josephson ME, Basford WP, Weisfogel GM, Lau SH, Damato HN. The electrophysicology of propranolol in man. An Heart J 1974, 88:733-741.

328. Seipel I., Bicithardt G, Dohring IIP. Die Wirkung von Atenolol auf den Sinusknoten und die intrakardiale Erregungsteitung beim Menschen im Vergleich zu Propranolol. Z. Kardiol 1977. $60: 719$

329. Cruickshank JM, Frichard BNC. Adverse reactions. In: Beta-blockers in clinical practice. Cruiksslank JM, Prichard BNC. (eds). Edinburgh London Melboume and New York: Churchill Livingstone, 1987 pp823

330. Cruikshank IM. Prichard BNC. Pharmacodynamics In: Beta-blockers in clinical practice. Cruickshank JM, Prichard BNC (eds). Eslinburgh London Melbourne and New York: Churchill Livingstone, 1987:ppo2.93.

331. Chan TYK, WoO KS, Critchley JAIH, Swaminathan R, Nicholls MG. Effects of nebivolol on blood pressure, hormones and cardac function in chinese subjects with essential hypertension. Drug Invest 1991, 3(suppl 1):98-104.

332. Bulpitt CI \& Fietcher AE. The measurement of quality of life in hypertensive patients : a practicat approach. Br J Chin Plamacol 1990, 30:353-364t.

333. Wenger NK Quality of life issues in hypertension: consequences of diagnosis and considerations. in managenent. An Heart J 1988, $116: 628-632$.

334. Vandenturg MU. Bvans SJW, Kelly BJ, Bradshaw F. Curric WJC, Cooper WD. Factors affecting the reporting of symptoms by hyperiensive patients. Br ] Clin Pharracol 1984, 10:189-195.

335. Bulpitt Cl, Dollery CT, Carne S. Change in symptoms of hypertensive patients after referral to haspital clinic. Br Hear! ! 1976, 38:121:128.

336. Bengrsson C, Edstrom K, Furness B, Sigurdsson I, Tibblin G. Prevalence of subjectively experienced symptoms in a population sample of women with special reference to women with arterial hypertension. Scand J Prim Health Care 1987, 5:1-8.

337. MCCarthy ST, McCarthy GL, John SM, Gould SE. A placebo-controlled crossover study of ketanserin in elderly hypertensive patients. Cardiovase Drugs Ther 1990, 4:115-117. 
338. Hollenberg NK, Testa M, Williams GH. Quality of life as a therapeutic end-point. An analysis of therapeutic trials in hypertension. Drug Safety 1991.6:83-93.

339. Fetcher AE, Bulpitt CJ. Hawkins CM, Havinga TK. ten Berge BS, May IF, Schuurman FH, van der Veur E, Wesseling H. Quality of life on antihypertensive therapy : a randomized double-blind tral of captopril and atenolol. J Hypertens 1990, 8:463-460.

340. Tfelt-Hansen P. Efficacy of beta-blockers in migraine: a critical review. Cephalalgia 1986, 6(suppl 5): $15-24$

341. Steiner SS, Friedhoff AJ, Witson BL. Wecker JR. Santo JP. Antibypentensive therapy and quality of life: a comparison of atenolol. captopril, enalapril and propranolol I Hum Hypertens 1990 , $4: 217 \cdot 225$.

342. Spilker B. Introduction to the field of quality of tife trials. In Quality of life assessments in clinical trials. Spilker B (Ed). Riven Press New York, 1990, pp3-9.

343. Gillings $D$, Koch $G$. The application of the principle of intention-to-treat to the analysis of clinical rrials Drug Information Joumal 1991, 25:411-124

344. Palmer AJ, Fletcher AE, Rudge PJ, Andrews CD, Callaghan Ts, Bulpit CJ. Qualicy of life in hyperiensives treated with atenolol or captopril : a double-blind crossover trial. J11yperiens 1992 , 10. $1409-1416$.

345. Franz I-W, Lohmann FW, Koch G. Effects of chronic antihypenensive treatment with accbutolol and pindolol on blood pressures, plasma catecholamines and oxygen uptake at rest and during submaximal and maximal exercise. I Cardiovase Pharmacol 1982, 4:180-186.

346. Smyth GA, Hughson RL, Walters DJ, Ranney DA. Short-versus long-term effects of oral betablockers on exercise performance. J Cardiac Rehab 1984, 4:227.237.

347. Van Baak MA, Jennen W, Verstappen FTJ. Maximal acrobic power and blood pressure in normotensive subjects after acute and chronic administration of metoprolol. Eur I Clin Pharmacol 1985. $28: 143-148$.

348. Szlachcic J, Hirsch AT, Tubau JF, Vollmer C, Henderson S, Massic BM. Diltiazem versus propranolol in essential hypertension : responses of rest and exercise blood pressure and effects on exercise capacity. Am J Cardiol 1987, 59:393-399.

349. Van Baak MA, Koene FMM, Verstappen FTJ. Exercise hemodynamics and maximal exercise capacity during beta-blockade in normotensive and hypertensive subjects. Br $\mathrm{J}$ Clin Pharmacol $1988,25: 169-178$.

350. Sahlin K. Metabolic changes limiting muscle performance. In : Biochemistry of exercise V1. Saltin B (ed). International series on sport sciences, vol 16. Champaign: Human Kinetics Publisher, 1986:pp323-343.

351. Sanchez RA, Cianciulli T, Dopica AM, Gianonne C, Atamirano J, Ramirez AJ, Marco EJ. Effects of nebivolol on left ventricular function in patients with essential hypertension. Drug Invest 1991, 3 (suppl 1): $155-160$.

352. Collier CR. Determination of mixed venous $\mathrm{CO}_{2}$ tensions by rebreathing. J Appl Physiol 1956. 9:25-29.

353. Jones NL, Campbell EJM, McHardy GIR, Higgs BE, Clode M. The estimation of carbon dioxide pressure of mixed venous blood during exercise. Clin Sci 1967, 32:311-327. 
354. Van Herwarden CLA. Binkhorst RA. Fennis JFM, Van 't Laar A. Reliability of the cardiac output measurement with the indirect Fick principle for $\mathrm{CO}_{2}$ during exercise. Pflügers Arch 1980, $385: 21 \cdot 23$

355. Inman MD, Hughson RI., Jones NL. Comparison of cardiac output during exercise by singlebreath and $\mathrm{CO}_{2}$ rebreathing methods. J Appl Physiol 1985, 58:1372-1377.

356. Costill DI, Fink WJ. Plasma volume changes following exercise and thermal dehydration. I Appl Physiol 1974, 37:521.525.

357. Borg G. Perceived exertion as an indicator of somatic stress. Seand I Rehab Med 1970, 2:92-98.

358. Duncombe MG. The colorimetric micro-determination of non-esterified fatty acids in plasma. Clin Chim Ac1a I964، 9:122-125

359. Weddle OH, Amick EN, Mason WD. Rapid determination of atenolol in plasma and urine by highperformance liquid chromatography. J Pharm Sci 1978, 67:1033-1035

360. Woestenborghs R, Embrechts 1., Heykants ). HPLC fluorescence method for the determination of the new beta-1 adrenoceptor biocking agent nebivolol in human plasma. In: Bioanalysis of drug and metaholites, especially anti-inflammatory and cardiovascular. Reid E et al (eds). Volume 18 of the series "Methodological assays in biochemistry and analysis". Plenum Press, New York, $1988 \mathrm{pp} 215-216$.

361. Zerbe GD. Fandomization analysis of randomized blocks design extended $t 0$ growth and response curves. Commun Stat Theor Met 1979, A8:191-205

362. Laurence DR. Bennet PN. Incividual or biological variation. In: Clinical Phamacology Laurence

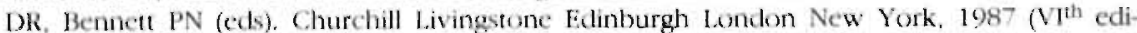
tion) : $p$ pl $36 \cdot 144$.

363. Van Baak MA. Beta-adrenoceptor biockade and exercise. An update. Sports Med 1988, 4:209-225.

364. Mooi\} J, Van Baak M, Botm R, Does R, Petri H, Van Kemenade J, Rahn KH. The effects of verapamil and propranolel on excrcise tolerance in hyperiensive patients. Clin Pharmacol Ther 1987. 41:490-495.

365. v Baak MA, Mooij JMV. The influence of glucose infusion on endurance performance after beta. blocker administration. Med Sci Spons Exerc 1991, 23(suppl): $\$ 77$.

366. Galbo H. Christensen NI. Holst II. Glucose-induced decrease in glucagon and epinephrine responses to exercise it: m.tn. J Appl Physiol 1977, $42: 525-530$.

367. Van Baak MA, Mooij JMV, Wijnen JAG, Tan ES Submaximal endurance exercise performance during enalapril treatment in patients with essential hypertension. Clin Pharmacol Ther 1991 . $50: 221 \cdot 227$.

368. Van Baak MA. Koene FMM, Verstappen FTJ, Tan ES. Exercise performance during captopril and atenolol treatment in hypertensive patients. Br J Clin Pharmacol 1991. 32:723-72.8.

369. Van Baak MA, Verstappen FTJ, Oosterhuis B. Twenty-four hour effects of oxprenolol oros and atenolot on heart rate, blood pressure, exercise tolerance and perceived exertion. Eur J Clin Pharmacol 1986, 30:399-406.

370. Katz A, Sahlin K, Juhlin-Dannfelt A. Effect of beta-adrenoceptor blockade on $\mathrm{H}^{*}$ and $\mathrm{K}$ * flux in exercising humans. J Appl Physiol 1985, 59:336-341 
371. Kullmer T, Kindermann W. Physical performance and serum potassium under chronic betablockade. Eur J Appl Physiol 1985, 54:350-354.

372. Leenen FH, Coenen CHM, Zonderland M. Maas AHJ. Effects of cardioselective and non-selective beta-blockade on dynamic exercise performance in mildly hypertensive men. Clin Pharmacol Ther 1980, 28:12-21.

373. Sipgaard G, Adams RP, Saltin B. Water and ion shifts in skeletal muscle of hunans with intense dynamic knce extension. Am J Physiol 1985, 248:R190-R196.

374. Management Committec of the Australian Therapeutic Trial in Mild Hypertension. Untreated mild hypertension. Lancet 1982, 1:185-191.

375. Mac Mahon SW, Cutler JA, Furberg CD, Payne H. The effects of drug treatment for hypertension on morbidiry and mortality from cardiovascular disease: A review of randomized controlled trials. Prog Cardiovasc Dis 1986, 29(suppl 1):99-118.

376. Flercher AE, Bulpit C. How far should blood pressure be lowered? N Engl J Med 1992 , $326: 251 \cdot 254$.

377. Beevers DG. Overtreating hypertension. Br Med J 1988, 297:1212-1213.

378. British Hypertension Society Working Pariy. Treating mild hypertension. Br Med J 1989, $298: 694-698$.

379. Zancheti A, Amery A, Berglund G, Cruickshank JM, Hansson L, Lever AF, Sleight P. How much should blood pressure be lowered? The problem of the f-shaped curve. I Hypertens 1989; 7 (suppl 6):\$338-5348.

380. Farnett L, Mulrow C, Linn WD, Lucey CR, Tuky MR. The J-curve phenomenon and the treatment of hypertension: is there a point beyond which pressure reduction is dangerous? JAMA 1991, $265: 489-495$.

381. Alderman MH. Meta-analysis of hypertension treatment trials. Lancet 1990, 335:1092-1093.

382. Kaplan NM. Meta-analysis of hypertension treatment trials. Lancet 1990, 335:1093.

383. Coope J. Warrender TS. Lowering blood pressure. Lancet 1987, 2:518.

384. Kanne] WB, Sorlie P, Castelli WP, McGee DI. Blood pressure and survival aftes myocardial infaretion: the Framingham Siudy. Am J Cardiol 1980, 45:326-330.

385. Coope J, Warrender TS, McPherson $\mathrm{K}$. The prognostic significance of blood pressure in the elderly. J Hum Hypertens 1988, 2:79-88.

386. Houston MC. New insights and approaches to reduce end-organ damage in the treatment of hyperiension: subsets of hypertension approach. Am Hear y 1902, 123:1337-1367.

387. Tift CP, Chobanian AV. Are some antihypertensive therapies more efficacious than others in preventing complications and prolonging life? Hypertension 1991, 18(suppl 1):1146-1152.

388. Gordon T, Sorlic P, Kannel WB. Coronary hean disease, atherothiombotic brain infarction, intermittent claudicatio - A multivariate analysis of some factors related to their incidence; 16 yeas follow-up. In: The Framingham Study, an epidemiologic investigation of cardiovascular disease. Appendix B, Washington DC, US Government Printing Office, 1971, section 27. 
389. Wassertheil-Smoller S, Oberman A, Blaufox MD. Davis B. Langford H. The Trial of Antihypertensive Interventions and Management (TAIM) Study. Final essults with regard to blood pressure, cardiovascular risk, and quality of life. Am J Hypertens 1992, 5:37-44.

390. Northcesc RI. Metabolic parameters: how important are phamacologically-induced changes? Jllypertens 1991, (suppl 7):\$21-525.

39). Yusuf S, Peto R, Lewis J, Collins R, Sleight P. Beta-blockade during and after myocardial infarcbion: an overview of the randomized trials. Prog Cardiovasc Dis 1985, 27:335-371.

392. Cruickshank JM. The case for beta-blockers as first-line antihypertensive therapy. J Hypertens 1902, $10($ suppl 3) $52.1-527$.

393. Management Commuttec of the Australian Therapeutic Trial in Mikd Hyperiension. The Australian Therapeatic Trial in Mile Hypertension Lancel 1980, 1:1261-1271.

394. Veterans Administration Cooperative Study Group on Anthypertensive Agents. Effect of treatment on morbidity in bypenension. If Results in patients with diastolic blood pressure averaging 90 through $119 \mathrm{mmH}$. JAMA 1970, 213:1143-1152.

395. Lerea P. Helgeland A. Coronary heart disease and treatment of hypertension: some Oslo Study data. $\mathrm{Am}$ I Mcd 1986, 80:3-6.

396. Wikstrand J. Beta-bleckers and cardioprotection: is there any good news from the recent trials? J Chin Pharmacol Ther 1987, 12:347-350

397. Wiksirand J. Wamold 1, Oksen G. Tuomilehto J. Elmfedt D, Berglund G. Primary prevention Wibl) metoprolol in patients whih hypertension: mortality results from the MAPHY study. JAMA $1988.259: 1976.1982$

398. Amen: A, Bukenhiger W, Brixko P, Bulpitt C, Clement D, De Leeuw P, de Plaen JF, Deruyttere M, De Schaepdryver A, Dollery C, Fagard R, Feltamp H, Forete F, Forte J. Handy R, Henry JF, Koistinen A. Teoneti G, Lund-Johansen P, Morris J, Nissinen A, O'Brien E, O Malley K. Terzoli L, Tuomilehto y, Webster J, Williams B. Influence of antihypertensive drug treatment on morbidity and mortality in patients over the age of 60 yeass. EWPHE results sub-group analysis based on cotry stratification. J Hypertens 1986, 4(suppl 6):\$642-5647.

399. SIIEP Cooperative Research Group. Prevention of stroke by anthypertensive drug treatment in olite persons wilh isolated systolic hypertension: final results of the Systolic Hypertension in the Bkerly Program (SHI:P). JAMA 1901, 265:3255-3264.

400. MRC Working Party. Medical kesearch Council trial of treatment of hypertension in older adults: principal results. Bi Med ] 1992, 304:405-412.

401. Beto JA, Bussal VK. Quality of life in treament of hypertension. A metaanalysis of clinical trials. Am I Hyperiens 1992, 5:125:133.

402. Linz W. Scholkens BA, H.un YF. Beneficial effects of the converting enzyme inhibitor ramipril in ischaemic fat heat. I Cardiovasc Pharmacol 1986, 8(suppl 10):591-599.

403. De Graeff PA, Van Gitst WII. Bet KI. De Langen CD, Kingma JH, Wesseling H. Concentration. dependent protecion by captopril against myocardial damage during ischaemia and reperfusion in a closed-chest pig model. J Cardiovasc Pharmacol 1987, 9(suppl 2):S37-S42.

4.04. Kingma Ji, De Graeff PA, Van Gilst WH, Van Binsbergen E, De Langen CD, Wesseling H. Effects of intravenous captopril on inducible sustained ventricular tachycardia one week after experimental infarction in the anaesthetized pig. Postgrad Med J 1986, 62(suppl 1):159-163. 
405. De Langen CDJ. De Graeff PA, Van Gilst WH. Effects of angiotensin 11 and captoprit on inducible sustained ventricular tachycardia two wecks after myocardial infarction in the pig. I Cardiovasc Pharmacol $1989,13: 186,191$.

406. Pollare T. Lithell H. Beme C. A comparison of effects of hydrochlorothiazide and aptoprit on glucose and lipid metabolism in patients with hypertension. N Fngl I Med 1989, 32 1 :868-873.

407. Insta A. Ribstein 1. Mimran A. Comparative effect of captopril and nifedipine in normotensive patients with incipient diabetic nephropathy. Postgrad Med I 1988, 64(suppl 3):59-62.

408. Fletcher AE, Bulpitt CJ. Chase DM. Collins WC, Furberg CD, Goggin TK, Hewetl Al, Neiss AM. Quality of life with three antihypertensive treaments : cilazapril, atenolol, nifedipine. Hypertension $1992,19: 499-507$.

409. Yusuf S. Held P. Furberg C. Update of effects of calcium antagonisis in myocardial infarction or angina in light of the second Danish Verapamil Intarction Trial (DAVIT II) and other recent sudies. Am J Cardiol 1991, 67:1295-1297.

410. Glasser SP, Clark PI, Lipicky RJ, Hubbard jM, Yusef A. Exposing patients with chronic, stable ex. ertional angina to placebo periods in drug trials. JAMA 1991, 265:1550-1554.

411. The Holland Interuniversity Nifedipine/Metoprolol Trial (HINT) Research Group), Early ureatment of unstable angina in the coronary care unit: a randomized double-blind, placebo controlled comparison of recurrent ischaemia in patients treated with nifedipine of metoptolol or both. Br Heart J 1986, 56:400-413.

412. Sorkin EM. Clissold SP, Brogden RN. Nifedipine: a review of is pharmacodymamic and phamacokinetic propertics, and therapeutic efficacy, in ischemic heart disease, hypertension, and related cardiovascular clisorders. Drugs $1985,30: 182-274$.

413. Kleinbloesem $\mathrm{CH}$, van Brummelen P. Danhof $M$, Faber H, Urquhart J, Breiner DD. Rate of increase in the plasma concentration of nifedipine as a major determinant of its hemodynamic effects in humans. Clin Pharmacol Ther 1987, $41: 26-30$.

414. Anonymous. Nifedipine: a new life with GITS? Lancet 1992, 340: $1507-1508$.

415. Talseth T, Westlic L, Daae L.. Doxazosin and atcnolol as monotherayy in mild and moderate hypertension: a randomized parallel study with a three-ycar follow-up. Ain Heart $\mathrm{J} 1991$, $121: 280-285$.

416. Hernandez RH, Carvajal AR, PajueloJG, Hernandez MJA, Padilla MCA, Barragan O, Boada JJB, Roa E. The effect of doxazosin on platelet aggregation in normotensive subjects and patients with hypertension: an in vitro study. Am Heart J 1991, $121: 389-394$.

417. Jansson JH, Johansson B, Boman K, Nilsson TK. Effects of doxazosin and aterolol on the fibrinolytic system in patients with hyperiension and elevated serum cholesterol. Eur J Clin Pharmacol 1991, 40:321-326.

418. IPPPSH Collaborative Group. Cardiovascular risk and risk factors in a randomised trial of treatment based on the $\{$ blocker oxprenolol : the International Prospective Primary Prevention Study in Hypertension (IPPPSH). J Hypertens 1985, 3:379-392.

419. Coope J, Warrender TS. Randomised Trial of hypertension of elderly patients in primary care. Br Med J 1986, 293:1145-1151.

420. Cruickshank JM, Pennert K., Sorman AE, Thorp JM, Zacharias FM, Zacharias F. Low mortality from all causes, including myocardial infarction, in well-controlled hypertensives treated with a beta-blocker plus other hypertensives. J Hypertens 1987, 5:489-498. 
421. Norwegian Study Group. Timolot-induced reduction in mortality and reinfarction in patients surviving acute myocardial infarction. N Engl f Med 1981, 304:801-807.

422. Beta Blocker Heart Attack Study Group. The Beta Blocker Heart Artack Trial. JAMA 1981, $246: 2073 \cdot 2074$.

423. Beta Blocker Jleart Attack Trial Research, Group. A randomized trial of propranolol in patients with acute myocardial infaretion: 1. Mortality results. JAMA 1982, 247:1707-1714.

424. Olssen G, Lubsen J, van Es G, Rechnquist N. Quality of life after myocardial infarction: effect of long-term metoprolol on mortality and morbidity. Br Med J 1986, 292:1491-1493.

425. Matl WE, Greenberg 6. Mild hypertension: Is these pressure to treat? An account of the MRC Trial Cambridge: Cambridge I niversity Press 1987.

426. Cruickshank JM. \& bleckers and primary prevention revisited. Cardiovase Rev Rep 1990, $11: 10 \cdot 16$

427. Fox FM, Chopra MP, Portal RW, Aber CP. Long-term beta-blockade: possible protection from myocardial infarction. Br Med ] 1975, 1:117-119.

428. Nidorf SM, Parsons RW. Thompson PL. Jamrozik KD. Hobbs MST. Reduced risk of death at 28 days in patiens taking a beta-blocker before admission to hospital with myocardial infarction. Br Med 1 1900, 300:71-74.

429. Frishman W1I, Chang CM. Beta-adienergic blockade in the prevention of myocardial infarction: a new theory. J IIypertens, 1991, 9(suppl 7):S31-S34.

430. Kjekshus IK. Importance of heart rate in determining beta-blocker efficacy in acute and long-term acute myocardial intervention trials. Am I Cardiol 1986, 57:43F-49F.

431. Kannel WB, Kannel C, Paffenbarger RS, Cupples LA. Heart rate and cardiovascular mortality: the Framingham Study Am Heart 1 1987, 113:1489-1494.

432. Regitz V, Lerchs B, Welnhofer E, Krults-Munch J, Fleck E. Improvement in hear failure with long $1 \mathrm{erm}$ ACE inhibitor and \&-blocker therapy is comparable. Circulation 1992, 86(suppl I):1-1 19 abstr 474.

433. Croog SH, Levine S, Testa MA, Brown B, Bulpitt Cl. Jenkins CD, Klerman GI., Williams GH. The eftects of antihypertensive therapy on the quality of life. N Engl J Med 1986, 314:1657-1664.

434. Case DB. Patient population as consideration for antihypertensive therapy: in: Management of hypertension: a multifictorial approach. Hollenberg NK (ed). Butterworth Publishers Stoneham MA 1987:pp101-120.

435. Hollenberg NK. Treatment of hypertension: The place of angiotensin-converting enzyme inhibitors in the nineties. I Cardiovasc Pharnacol 1992, 20(suppl 10):S29-532.

436. Bennet DH. Cardiac arrhythmias. In: Oxford textbook of medicine. Weatherall DJ, Ledingham JGG, Warrel! DA (eds). Oxford university press, Oxford New York Toronto I985:pp13.78-13.92.

437. Ledingham JGG. Diuretics. In: Oxford textbook of medicine. Weatherall DJ, Ledingham JGG, Warrell DA (eds). Oxford university press, Oxford New York Toronto 1985:pp13.61-13.63.

438. Schick FL. Statistical hạdbook on aging Americans. Oryx press, Phoenix AZ 1986:p109.

439. Kaplan NM. Guidelines for the treatment of hypertension. Am J Hypertens 1989, 2:75-77. 
440. Kannel WB. Bishop lecture: Contribution of the Framingham sudy to preventive cardiology. J Am Coll Cardiol 1990, 15:200-211.

441. Ridker PM, Hennekens CH. Hemostatic risk factors for coronary hear disease. Circulation 1991. $83: 1098-1100$.

442. Heller RF, Chinn S. Tunstall Pedoe HD, Rase G. How well can we prediet coronary hean disease? Findings in the United Kingdom Hean Disease Prevention Project. Br Med J 1984, 288: $1409-1411$.

443. Christensen KL. A reduction in cyclic pressure loading may be impontant for the effect of antihypertensive treatment on resistance vessel structure. Blood vessels 1991. 28:280.

444. Paoleti R, Corsini A, Soma M. Fumagalli R. Direet effects of statins on smooth muscle cells in arterial walls. Relation to the progression of atheroselerosis. Abstractbosk of the Vth Workl Conference on Clinical Pharmacology and Therapeutics 1992:p70.

445. Mulvany MJ. The development and regression of vascular hypertrophy. J Cardiovasc Pharmacol 1992, 19 (suppl 2):\$22-S27.

446. Baumbach GL, Heistad DD. Renodeling of cerebral arterioles in chronic hypertension. Hyperten. sion 1989, 13:968972.

447. Vander Stichele $\mathrm{R}$. Measurement of patient compliance and the interpretation of randomized trials. Eur J Clin Pharmacol 1991, $41: 27-35$.

448. Pullar T. Kumar S, Tindall H. Feely M. Time to stop counting the tablets? Cin Pharmacol Ther $1989,46: 163-168$

449. Anonymous. Patient compliance in therapeutic trials. Lancet 1991, 337:823-824.

450. Leventhal II. The role of theory in the study of adherence to treatment and doctor-patient interactions. Med Care 1985, 23:556-563

451. Bond WS, Hussar DA. Detection methods and strategies for improving medication compliance. Am J Hosp Pharm 1991, 48:1978-1988.

452. Maënpäi $H$, Manninen $V$, Heinonen OP. Comparison of the digoxin marker with capsule counting and compliance questionnaire methods for measuring compliance to medication in a clinical trial. Eur Heart J 1987, 8(suppl 1):39-43.

453. Rudd P, Byyny RL, Zachary V, LoVerde ME, Titus C, Mitchell WD. Marshall G. The natural history of medication compliance in a drug trial : Limitations of pill counts. Clin Pharmacol Ther 1989, $46: 169-176$.

454. Urquhart J Non-compliance: the ultimate absorption barrier. In: Prescoll LF, Nimmo WS (eds). Novel drug delivery and its therapeutic applications. John Wiley and Sons 1989, New York.

455. Pullar T, Birtwell AJ, Wiles PG, Hay A, Feely MP. Use of a pharmacologic indicator to compare compliance with tablets prescribed to be taken once, twice, or three times daily. Clin Pharmacol Ther $1988,44: 540-545$.

456. Urquhart J. Patient compliance as an explanatory variable in four selected cardiovascular studies. In: Cramer JA, Spilker B (eds). Patient compliance in medical practice and clinical trials. Raven Press 1991, New York. 
457. Kruse W, Eggert-Kruse W, Rampmaier J, Runnebaum B. Weber E. Dosage frequency and drugcompliance behaviour - a comparative study on compliance with a medication to be taken twice or four times daily. Eur J Cin Pharmacol 1991, $41: 589.592$.

458. Cramer JA, Mattson RII, Prevey ML, Scheyer RD. Ouclette VL. How often is medication taken as prescribed? A nesvel assessment technique. JAMA. 1989, 261:3273-3277.

459. Kruse W, Schilicr G, Weber E. Monitoring compliance in clinical trials. Lancet 1990 , $335: 803-804$ 


\section{SUMMARY}

Prevalence of hypertension is high. In most hypertensive patients, the real cause of hypertension is not known (essential hypertension). However, there is much evidence that alterations in small vessels (the microcirculation) play an important role in the onset of most cases of essential hypertension. A minority of hyperten. sive patients have severe hypertension, which might very quickly lead to death (malignant hypertension). Most patients have mild-to-moderate hypertension, which is, in general, asymptomatic. This class of hypertension can in the long run lead to cardiovascular disease and renal failure. The complications of hypertension are predominantly due to disease of the large vessels (macrocirculation). Therefore, prevention or retardation of these late complications [1] of hypertension has to be the goal of antihypertensive treatment in mild-to-moderate hypertension. This is only in part achieved by treatment of the high blood pressure as such.

The outcome of treatment depends largely on patient compliance to that treat ment. Since mild-to-moderate hypertension is asymptomatic, small adverse effects of a drug can easily decrease patient's quality of life [2] and lead to a lower patient compliance.

This thesis pays attention to the above mentioned $[1,2]$ non-antihypertensive aspects of antihypertensive treatment. Part two deals with the influence of physical factors on the vessel wall of large arteries, which might play a role in the appearance of late complications of hypertension. Part three investigates the influence of different antihypertensive treatments on patients' quality of life.

Chapter I of part 2 reviews different physical factors and their determinants (such as distensibility and compliance, shear stress and pulse wave reflections). Distensibility and compliance are vessel wall properties. Distensibility reflects the elastici. ty of the vessel wall, while compliance is a measure for the buffering capacity of the vessel. This chapter also reviews the influence of antihypertensive therapy on physical factors and their determinants. Not all antihypertensive drugs can improve vessel wall properties of large arteries. Calcium-antagonists, ACE-inhibitors and selective $\beta_{1}$-blockers improve vessel wall properties, while nonselective $\beta_{\text {-blockers }}$ do not. Chapter 2 and 3 investigates the effect on vessel wall properties of the common carotid artery of the calcium-antagonist verapamil and the selective $\aleph_{\text {- }}$-blocker with vasodilating properties nebivolol, respectively. Both drugs improved distensibility and compliance of the common carotid artery. This improve ment might postpone cardiovascular complications of hypertension.

Recently, increasing attention is being paid to the influence of drugs on the quality of life. Several questionnaires have been developed to measure quality of life more adequately. One of these questionnaires, the Inventory of Subjective Health (ISH), has becn developed in The Netherlands and is presented in the introduction of part 3. Chapter 1 of part 3 investigates the influence of antihypertensive therapy with nebivolol (a selective $\beta_{1}$-blocker with vasodilating properties) on blood pressure and quality of life. Nebivolol $5 \mathrm{mg}$ once daily induced a decrease in blood pressure, similar to that of other antihypertensive drugs, given as monotherapy. No drug tolerance was seen during the 8-week treatment period. Nebivolol did not impair quality of life. In addition, the study showed that participation in a trial by itself already improves quality of life. Therefore, comparison of quality of life 
during treatment with quality of life before treatment does not allow conclusions about a beneficial effect of a drug on quality of life.

Chapter 2 compares the influence on quality of life of antihypertensive treatment with the ACE-inhibitor enalapril and the selective $B_{1}$-blocker bisoprolol. At dosages, which induced a comparable decrease in blood pressure, less adverse effects were mentioned with bisoprolol than with enalapril. Quality of life, measured with the Inventory of Subjective Health, tended to be better with bisoprolol and the large majority of patients preferred continuation of treatment with bisoprolol. These results clearly do NOT support the assumption that ACEinhibitors induce less complaints than B-blockers, and certainly not less than selective $B_{1}$-blockers.

B-Blockers may decrease quality of life in physically active patients by decreasing exercise tolerance. Endurance exercise capacity decreases by about $40 \%$ with nonselective B-blockers, by $20-25 \%$ with selective $B_{1}$-blockers, and by $8-12 \%$ with ACE-inhibitors and calcium-antagonists. Chapter 3 investigates the influence on exercise capacity of ncbivolol $5 \mathrm{mg}$ once daily compared to atenolol $100 \mathrm{mg}$ once daily and placebo. Decrease in blood pressure was comparable during nebivolol and atenolol, but heart rate decreased less with nebivolol, suggesting less B-blockade with nebivolol. Endurance time decreased by $23 \%$ with atenolol, while only by $6 \%$ with nebivolol. Decrease in endurance time with nebivolol was similar to that with calcium-antagonists and ACE-inhibitors, and this decrease, in general, does not induce complaints of fatigue.

Part 4 discusses different aspects of current antihypertensive therapy. A preference for treatments with proven beneficial effect on morbidity and mortality is suggested above treatments with (based on their beneficial effects on risk factors) expected, but not proven, beneficial effect on morbidity and mortality. Finally, issues for future research are proposed such as further evaluation of current antihypertensive therapy, exploration of prevention and possible reversibility of ill effects, 24-hour protection, identification of patients at risk, and, although not limited to antihypertensive treatment, control of patient compliance. 


\section{SAMENVATTING}

Hypertensie is een vaak voorkomende aandoening. In de meerderheid der gevallen is haar oorzaak niet goed bekend. Deze vorm van hypertensic wordt essentiele hypertensie genoemd. Toch zijn er aanwijzingen dat in vele gevallen afwijkingen in de kleine bloedvaten (de microcirculatie) een belangrijke rol spelen bij het ontstaan van essentiële hypertensie. In een kleine minderheid van de patienten bestaat een sterk verhoogde bloeddruk die snel tot de dood kan leiden (maligne hypertensie). De meeste patiënten hebben echter een licht tot matig verhoogde blocddruk en ondervinden hiervan in de regel geen hinder. Deze vorm van hypertensie kan op den duur toch leiden tor hart- en vatziekten en nierfalen. De complicaties van de hypertensie situeren zich vooral in het hat en de grote vaten (macrocirculatie). De behandeling van hoge bloeddruk moet darom vooral gericht zijn op het voorkomen of vertragen van deze late complicaties. Behandeling van de hoge bloeddruk alleen, blijkt hierin slechts ten dele te slagen.

Het succes van een behandeling hangt in grote mate af van de trouw warmee de voorgeschreven medicatie werd ingenomen (therapietrouw). Omdat lichte tot matige hypertensie meestal symptoomloos is, kunnen geringe bijwerkingen van een medicijn de levenskwaliteit van een patiënt snel negatief beinvloeden en tot een lagere therapietrouw leiden.

In dit proefschrift wordt vooral aandache besteed aan de genoemde nietantihypertensieve aspecten van de behandeling van de hoge bloeddruk. Deel 2 behandelt de invloed op de vaatwand van grote vaten van fysische factoren, die een rol kunnen spelen bij het ontstaan van de late complicaties van hypertensie. Decl 3 onderzoekt de invloed van verschillende antihypertensieve behandelingen op de levenskwaliteit van de patient

Hoofdstuk I van deel 2 geeft een overzicht van de verschillende fysische factoren en hun determinanten, die een invloed kunnen hebben op de vaatwand (zoals distensibiliteit en compliantie, afschuifspanning en polsgolfreflecties). Distensibiliteit en compliantie worden ook vaatwandeigenschappen genoemd. De distensibiliteit is een maat voor de elasticiteit van de vaatwand, terwijl de compliantie het bufferende vermogen van het bloedvat weergeeft. Een overzicht van de invloed van verschillende bloeddrukverlagende medicijnen op de valwandeigenschappen toont aan dat niet alle bloeddrukverlagende middelen de valatwandeigenschappen verbeteren. Een verbetering werd beschreven met calciumantagonisten, ACE-remmers en $\AA_{1}$.selectieve blokkeerders, maar niet met aselectieve B-blokkeerders. Hoofdstuk 2 en 3 beschrijven het effect op de valwandeigenschappen van de arteria carotis van respectievelijk de calciumantagonist verapamil en van nebivolol, een $\beta_{1}$-selectieve blokkeerder met vasodilaterende eigenschappen. Met beide middelen verbeterde de distensibiliteit en compliantie van de halsslagader. Het is mogelijk dat hierdoor cardiovasculaire complicaties van hypertensie worden vertraagd of vermeden.

De laatste jaren is in toenemende mate aandacht besteed aan de invloed van medicijnen op de levenskwalitiet van de patiënt. Diverse vragenlijsten werden ontwikkeld om de levenskwaliteit adequat te meten. Een van deze vragenlijsten, de VOEGlijst (vragenlijst onderzoek ervaren gezondheid), werd in Nederland ontwikkeld en wordt in de introductie van deel 3 gepresenteerd. Hoofdstuk 1 van deel 3 
onderzockt de invloed van een antihypertensieve behandeling met nebivolol (selectieve $B_{j}$-blokkeerder met vasodilaterende eigenschappen) op de bloeddruk en de kwaliteit van leven. Nebivolol $5 \mathrm{mg}$ eenmaal daags gaf een goede bloeddrukdaling, vergelijkbaar met die van andere $\mathbb{B}$ blokkeerders in monotherapie. Gedurende de 8 weken durende studie trad geen tolerantic op. Nebivolol had geen negatieve invloed op de kwaliteit van leven. De studie toonde ook aan dat deelname aan een onderzoek op zichzelf de levenskwaliteit al verbetert. Daarom kan de vergelijking van de levenskwaliteit tijdens met die van vóór de behandeling geenszins een positieve invloed van een medicijn op de levenskwaliteit aantonen. Hoofdstuk 2 vergelijkt de invloed op de kwaliteit van leven van een antihypertensieve behandeling met de ACE-remmer enalapril met een behandeling met de $B_{i}$-selecticve blokkeerder bisoprolol. Bij een vergelijkbare bloeddrukdaling werden minder bijwerkingen gesignaleerd tijdens behandeling met bisoprolol dan tijdens cnalapril. Ook was (niet significant) de levenskwaliteitscore van de VOEG. lijst beter met bisoprolol en gaf een ruime meerderheid van de patienten de voorkeur aan het verderzetten van de behandeling met bisoprolol. Deze resultaten ondersteunen duidelijk NIET de aanname dat ACE-remmers minder klachten geven dan $B$-blokkeeiders, en zeker nict minder dan $B_{1}$-selectieve blokkeerders. Het is bekend dat is-blokkeerders de kevenskwaliteit van fysisch actieve patiënten duidelijk kunnen verminderen door vooral het uithoudingsvermogen van de patient te beperken. Gemiddeld neemt het uithoudingsvermogen voor lichamelijke inspanning met ongeveer $40 \%$ af bij behandeling met niet-selectieve B-blokkeerders. Met selectieve B-blokkeerders werd een vermindering van $20-25 \%$ gevonden, terwijl dit met ACE-remmers en calciumantogonisten 8-12\% bedrocg. In hoolstuk 3 werd het uithoudingsvermogen tijdens een behandeling met nebivolol $5 \mathrm{mg} 1 \mathrm{x}$ daags vergeleken met dat tijdens een behandeling met atenolol $100 \mathrm{mg} 1 \mathrm{x}$ daags en placebo. De bloeddrukdaling was vergelijkbaar tijdens nebivolol en atenolol, maar de daling in hartrequentie was minder groot met nebivolol. Dit suggereert minder \& blockade met nebivolol. Tijdens atenolol werd een daling van de volhoutijd van $23 \%$ gevonden, terwijl deze slechts $6 \%$ bedroeg met nebivolol. De daling van de volhoutijd met nebivolol ligt daarmee in dezelfde grootorde van die met calciumantagonisten en ACE-remmers en dit geeft in de regel kennelijk geen aanleiding tot vermoeidheidsklachten.

In deel 4 worden verschillende aspecten van de huidige antihypertensieve therapie bediscuteerd. Hierbij wordt gepleit voor een voorkeur voor behandelingen met een bewezen gunstig effect op morbiditeit en mortaliteit boven behandelingen mer (op grond van hun gunstige invloed op risicofactoren) verwacht, maar niet bewezen, gunstig effect op morbiditeit en mortaliteit. Tenslotte worden velden voor verder onderzoek angegeven zoals verdere evaluatie van de huidige antihypertensieve therapie, onderzoek naar preventie en eventuele omkeerbaarheid van complicaties van hypertensic, 24-uurs protectic, identificatic van risicopatienten en, alhoewel niet specifiek voor behandeling van hypertensie, het meten van de therapietrouw van de patiënt. 


\section{CURRICULUM VITAE}

1949,

18 november

geboren te Kalmthout, Belgie

1961.1967 Griekinatijns humaniora an her Sint-Michiclscollege te Brasschaat, België.

1967-1970 Kandidaatsjaren geneeskundige wetenschappen Rijksuniversitair Centrum Antwerpen, België.

1970-1974 Genecskunde studie Rijksuniversiteit Gent, België.

1974 diploma doctor in genees-, heel, en verloskunde (Rijksuniversiteit Gent).

1974-1976 Assistent interne geneeskunde in het AZ Stuivenberg te Antwerpen, België.

1976-1977 postgraduaat cursus Tropische Geneeskunde aan het Instituut voor Tropische Geneeskunde te Antwerpen, België.

1977-1978 assistent chirurgie-gynaecologie en verloskunde in het Sint-jans. gasthuis te Weert, Nederland.

1978-1980 assistent van het Instituut voor Tropische Geneeskunde Antwerpen met opdracht in Kasongo, Zaire.

1980-1985 assistent in opleiding Interne Geneeskunde in het De Weverziekenhuis te Heerlen, Nederland.

erkenning als internist.

1985-1988 assistent klinische farmacologie, vakgroep Farmacologie (Prof. dr. K.H. Rahn en Prof. dr. H.A.J. Struijker Boudier) Rijksuniversiteit Limburg, Maastricht, Nederland.

1986-heden 20\% gedetacheerd als internist aan het De Wever ziekenhuis Heerlen, afdeling Interne Geneeskunde.

1989-heden werkleider sectie klinische farmacologie, vakgroep farmacologie (Prof. dr. H.A.J. Struijker Boudier) Rijksuniversiteit Limburg, Matastricht.

erkenning als internist met aandachtsgebied klinische farmacologie. 



\section{Lijst van publicaties}

\section{Artikels}

1. LMAB Van Burtel, C van Deursen. Schistosomiase als imponzickte. Mol Med $7,1007-1014,1983$

2. KH Rahn, LM Van Bortel, IM Mooij. The use of calcium antagonists in patients with renal failure. J Hypertoth 5 (suppl 4), 67-69, 1987

3. C van Deursen, L. Van Bortel, R Nikkels. Trombopenic bij malaria. Tijdsche Geneeskd 44, 1529-1532. 1988

4. JMV Mooij, L Van Bortcl, B Janevski. Een patient met ernstige hypertensic. Hart Bulletin 20, 122-123, 1989

5. L Van Bortel, R Böhm, J Mooij, P Schiffers, KH Rahn. Total and free steadystate plasma levels and pharmacokincrics of nifedipine in patients with terminal renal failure. Eur J Clin Pharmacol 37, 185-189, 1989

6. L Van Bortel, R Böhm, J Mooij, P Schiffers, KH Rahn. Plarmacokinetics of nitrondipine in terminal renal failure. Eur J Clin Pharmacol 36, 457-471, 1989

7. T van Merode, LM Van Bortel, FA Smeets, RS Reneman, R Böhm, KlI Rahn. Verapamil and nebivolol improve carotid artery distensibility in hypertensive patients. J Hypertens 7 (suppl 6), 262-263, 1989

8. T van Merode, L Van Bortel, F Smeets, R Böhm, J Mooij, KH Rahn, RS Reneman. The effect of verapamil on carotid artery distensibility and crosssectional compliance in hypertensive patients. J Cardiovasc Pharmacol 15, $109-113,1990$

9. HAJ Struijker Boudier, LMAB Van Bortel, JGR De Mey. Renodeling of the vascular tree in hypertension: drug effects. Trends Pharmacol Sci 11, 240-245, 1990

10. L Van Bortel, P Schiffers, R Böhm, J Mooij, KH Rahn, HAJ Struijker Boudier. The influence of chronic treatment with verapamil on plasma atrial natriuretic peptide levels in young and elderly hypertensive patients. Eur J Clin Pharmacol 39 (suppl 1), 39-40, 1990

11. LMAB Van Bortel, T van Merude, FAM Smeets, ROB Bohm, RS Reneman. Antihypertensive treatment and vessel wall properties of large arteries. Basic Res Cardiol 86 (suppl 1), 91-95, 1991 
12. JAG Wijnen, H Kuipers, MJF Kool, MA van Baak, HAJ Struijker Boudier, FTJ Verstappen, LMAB Van Bortel. Vessel wall properties of large arteries in trained and sedentary subjects. Basic Res Cardiol 86 (suppl 1), 25-29, 1991

13. LMAB Van Bortel, T van Merode, FAM Smeets, RS Reneman, JMV Mooij, HAJ Struijker Boudier. Nebivolol improves the vessel wall properties of the common carotid artery. Drug Invest 3 (suppl 1), 61-63, 1991

14. MJ Kool, JA Wijnen, AP Hocks, II Struijker Bouclier, LM Van Bortel. Diurnal pattern of vessel wall properties of large arteries in healthy men. J Hypertens 9 (suppl 6), 108-109, 1991

15. LMAB Van Bortel, MA van Baak. Exercise tolerance with nebivolol and arenolol. Cardiovasc Drugs Ther, 6, 239-247, 1992

16. IP Kooman, JAG Wijncn, P Draajer, L Van Bortel, U Glaclziwa, H Peltenburg, HAJ Struijker Boudier, JP van Hooff, KML Leunissen. Compliance and reactiv. ity of the peripheral venous system in patients with chronic intermittent dialysis. Kidney Int 41, 1041-1048, 1992

17. MJ Kool, HA Struijker Boudier, JA Wijnen, AP Hocks, LM Van Bortel. Effects of diurnal variability and exercise training on properties of large arteries.

J Hyperiens 10 (suppl (9), 49-52, 1992

18. LMAB Van Bortel, AP Hoeks, MJ Kool, HA Struijker Boudier. Introduction to large artery properties as a target for risk reduction by antihypertensive therapy. I Hypertens 10 (suppl 6), 123-126, 1992

19. JP Kooman, U Gladziwa, G Böcher, JAG Wijnen, LMAB Van Bortel, AJ Luik, PW de Leeuw, JP van Hooff, KML Leunissen. Blood pressure during the interdialytic period in hemodialysis patients: estimation of representative blood pressure values. Nephrol Dial Transplant 7, 917-923, 1992

20. JP Kooman, U Gladziwa, G Böcher, JAG Wijnen, LMAB Van Bortel, AJ Luik, PW de Leeuw, JP van Hooff, KML Leunissen. The role of the venous system in hemodynamics during ultrafiltration and bicarbonate dialysis. Nephrol Dial Transplant $42,718-726,1992$

21. L Van Bortel, Th de Vries, H van Rees, J Vollebregt. Farmacotherapie-onderwijs in Nederland. In: Gezond Onderwijs I (eds : CPM vd Vleuten, AJ]A Scherpbier, MC Pollemans). Bohn Stafieu van Loghum, pp 48-53, 1992

22. JGS Breed, R Ciampricotti, GP Tromp, FA. Valster, E Lageweg, LMAB Van Bortel. Quality of life perception during antihypertensive treatment. A. comparative study of Bisoprolol and Enalapril. J Cardiovasc Pharmacol 20, $750-755,1992$ 
23. LMAB Van Bonel, JGS Breed, J Joosten, JA Kragten, FATh Lustermans, JMV Mooij. Nebivolol in hypertension: A double blind placebo-controlled multicentre study assessing its antihypertensive efficacy and impact on quality of life. J Cardiovasc Pharmacol 21,856-862, 1993

24. LMAB Van Bortel, R Ciampricotti, GP Tromp, FA Valster, E Lageweg, J Joosten, FAT Lustermans, JGS Breed. Bisoprolol versus cnalapril; een dubbelblind vergelijkend onderzock nar de invloed op de kwaliteit van het leven. TGO/JDR 18, 151-155, 1993

25. JAG Wijnen, MA van Baak, C de Haan, HAJ Suruiker Boudier, FS Tan, LMAB Van Bortel. Beta-blockack and lipolysis during exercise. Eur J Clin Pharmacol $45,101-105,1993$

26. P Draaijer, MJ Kool, JM Maesen, LM Van Bortel, PW de Lecuw, JP van Hooff, KM Leunissen. Vascular distensibility and compliance in salt-sensitive and salt= resistant borderline hypertension. J Hypertens, in press

27. MJF Kool, APG Hexeks, HAJ Struijker Boudier, RS Reneman, I.AIAB Van Borcel. Acute and chronic effects of smoking on arterial wall propertices in halsiual smokirs. J An Coll Carliol, in press.

28. MJF Kool, FAT Lustermans, JGS Breed, HAJ Struijker Boudier, APG Hocks, LMAB Van Bortel. Effect of perindopril and amiloride/hydrochlorothiazide on hatemodynamics and vessel wall properties of large arteries. J Hypertens, in press

29. JA Wijnen, MJ Kool, JP Kooman, MA van Baak, HA Struijker Boudier, IM Van Bortel. Vessel wall properties of large arteries and endurance training.

$\mathrm{J}$ Hypertens, in press

30. J Vollebregt, L Van Bortel, Th de Vries, H van Rees. Ontwikkeling en toetsing van een meetinstrument voor het bepalen van de farmacotherapeutische vaardigheden van de basisarts. Gezond Onderwijs II, in press

31. JAG Wijnen, MJF Kool, MA van Baak, H Kuipers, CHA de Haan, FTJ Verstappen, HAJ Struijker Boudier, LMAB Van Bortel. Effect of exercise training on ambulatory blood pressure. Int J Sports $\mathrm{Med}$, in press 


\section{Abstracts}

1. LMAB Van Bortel, ROB Böhm, JMV Mooij, PMH Schiffers, HHW Thijssen KH Rahn. Plasma levels of nifedipine and nitrendipine in terminal renal failure. Pharm Whbld Sci $8,305,1986$

2. L. Van Bortel, R Böhm, J Mooij, P Schiffers, KH Rahn. The influence of age on the pharmacokinetics of verapamil given orally. Naunyn Schmiedeberg's Arch Pharmacol 335 (suppl), R105, 1987

3. LMAB Van Bortel, ROB Böhm, JMV Mooij, PMH Schiffers, HHW Thijssen, KH Rahn. Steady-state kinetics of nifedipine and nitrendipine in terminal renal failure. Proceedings 8th Dutch Federation Meeting, no 69, 1987

4. I.MAB Van Bortcl, ROB Böhm, JMV Mooij. PMH Schiffers, KH Rahn. Invloed van leeftijd op de plasmaspiegels van verapamil. Ned Tijdschr Geneeskd 131, 1548,1987

5. L.MAB Van Bortcl, ROB Böhm, JMV Mooij, PMH Schiffers, KH Rahn. Plasma levels of verapamil and age. Pharm Wkbld Sci 9, 188, 1987

6. LMAB Van Bortel, ROB Böhm, JMV Mooij, PMH Schiffers, HHW Thijssen, KH Rahn. Plasmaspiegels van nifedipine en nitrendipine bij terminale nierinsufficientic. Ned Tijdschr Geneeskd 131, 1369, 1987

7. R Böhm, T van Merode, L Van Bortel, F Smeets, R Reneman, KH Rahn. Untersuchungen der Gefässwandeigenschaften bei Hypertoniken mit Hilfe eines netuen gepulsten mehrkanaligen Dopplersystemis. Klin Wochenschr 66 (suppl 1.i), 430,1988

8. PMH Schiffers, LMAB Van Bortel, HAJ Struijker Boudier. Effect of verapamil on the secretion of atrial natriuretic peptide in young and elderly hypertensive patients. Pharm Wkbld Sci 10,233, 1988

9. L. Vin Bortel, T van Merode, FA Smeets, RS Reneman, KH Rahn. Effect of verapamil on the vessel wall properties of the carotid artery in man. Naunyn Schmiedeberg's Arch Pharmacol 338 (suppl), R82, 1988

10. MA van Baak, LMAB Van Bortel. Effects of the new beta-adrenoceptor antagonist nebivolol and atenolol during dynamic physical exercise. Proceedings International Symposium on Cardiovascular Disease and Beta-adrenoceptor Function, Antwerp, 1988

11. L. Van Bortel, KH Rahn. Effect of nebivolol on the response to isoprenaline in man. Proceedings International Symposium on Cardiovascular Disease and Beta-adrenoceptor Function, Antwerp, 1988 
12. L Van Bortel, J Joosten, H Breed, J Kragten, F Lustermans, J Mooij. Quality of life with nebivolol. Proceedings International Symposium on Cardiovascular Disease and Beta-adrenoceptor Function, Antwerp, 1988

13. L Van Bortel, P Schiffers, J Mooij. Invasive hemodynamics of nebivolol in hypertensive patients. Proceedings International Symposium on Cardio. vascular Disease and Beta-adrenoceptor Function, Antwerp, 1988

14. L. Van Bortel, H Breed, J Kragten, F Lustermans, J Mooij. Antihypertensive effect of nebivolol, a placebo-controlled multicenter study. Proceedings International Symposium on Cardiovascular Discase and Beta-adrenoceptor Function, Antwerp, 1988

15. L. Van Bortel, J Mooij, KH Rahn. Steady-state pharmacokinetics and dynamics of nebivolol, a novel beta-blocking agent. Proceedings International Symposium on Cardiovascular Disease and Beta-adrenoceptor Function, Antwerp, 1988

16. T van Merode, L Van Bortel, F Smeets, R Reneman. Effect of ncbivolol on the vessel wall properties of the carotid artery in hypertensive patients. Proceedings International Symposium on Cardiovascular Disease and Betaadrenoceptor Function, Antwerp, 1988

17. T van Merode, LM Van Bortel, FA Smeets, RS Reneman, KH Rahn. Effect of chronic administration of verapamil on the vessel wall properties of the carotid artery in man. Proceedings Symposium on Prospects and Strategies in Cardiovascular Research, Maastricht, 1988

18. FMM Koene, MA van Baak, JAG Wijnen, JJME Adam, L. Van Bortel. The influence of clonidine on exercise performance in healthy volunteers. Proceedings 12 th PUIJO Symposium on Physical Activity and Hypertension, 1989

19. RS Reneman, T van Merode, L Van Bortel, F Smeets, APG Hoeks. Arterial wall properties in hypertensive patients. The effect of therapy. FASEB Journal 3, 515,1989

20. MA van Baak, LMAB Van Bortel. Comparison of the new betaadrenoceptor antagonist nebivolol and atenolol during dynamic physical exercise. Proceedings 12 th PUIJO Symposium on Physical Activity and Hypertension, 1989

21. LMAB Van Bortel, ROB Böhm, JMV Mooij, KH Rahn. Plasma human ANP levels in young and elderly hypertensive patients treated with verapamil. Naunyn Schmiedeberg's Arch Pharmacol 339 (suppl), R1 16, 1989 
22. L. Van Bortel, R Böhm, J Mooij, P Schiffers, H Thijssen, KH Rahn. Steady-state plasma levels of nifedipine and nitrendipine in terminal renal failure. Proceedings XIX Congress of the International Union of Therapeutics, 1989

23. LMAB Van Bortel, PMH Schiffers, ROB Böhm, JMV Mooij, KH Rahn, HAJ Struijker Boudier. Verapamil alters plasma human ANP levels in hypertensive patients. Eur J Clin Pharmacol 36 (suppl), A339, 1989

24. LMAB Van Bortel, T van Merode, FA Smeets, RS Reneman, KH Rahn. Verapamil improves the vessel wall properties of the carotid artery in man. Neth J Med 35, A40, 1989

25. LMAB Van Bortel, T van Merode, FAM Smeets, RS Reneman, HAJ Struijker Boudier. Effects of nebivolol on the vessel wall properties of the common carotid artery in hypertensive patients. Proceedings 30th FEDERA Meeting, 1989

26. L. Van Bortel, P Schiffers, R Böhm, J Mooij, K Rahn, J-l Struijker Boudier. The influence of a chronic treatment with verapamil on plasma human ANP levels in young and elderly hypertensive patients. Alsstractbook 4 th European Meeting on Hypertension 93, 1989

27. LMAB Van Bortel, PMH Schiffers, ROB Böhm, JMV Mooij, KH Rahn, HAJ Struijker Boudier. Antihypertensive treatment with verapamil raises plasma ANP levels in man. Pharm Wkbld Sci suppl H, H6, 1989

28. LMAB Van Bortel, T van Merode, FAM Smeets, RS Reneman, HAJ Struijker Boudicr. Vessel wall properties of the common carotid artery in hypertension. Pharm Wkbld sci suppl K, K3, 1989

29. LMAB Van Bortel, T van Merode, FA Smeets, RS Reneman, RO Böhm, KH. Rahn. Effect of verapamil and nebivolol on the carotid artery distensibility in hypertensive patients. Eur Heart J 10 (suppl), 117,1989

30, T van Merode, IM Van Bortel, FA Smeets, RS Reneman, R Böhm, KH Rahn. Verapamil and nebivolol improve carotid artery distensibility in hypertensive patients. Abstractbook 4th European Meeting on Hypertension 559, 1989

31. LMAB Van Bortel, T van Merode, FAM Smeets, ROB Böhm, RS Reneman. Vessel wall propertics of the carotid artery in hypertensive patients treated with verapamil and nebivolol. Naunyn Schmiedeberg's Arch Pharmacol 341 (suppl), R59, 1990

32. LMAB Van Bortel, MA van Baak. Different exercise endurance performance but similar antihypertensive effect of two beta-blocking agents. Proceedings 31 th Durch liblekA Meeting 76, 1990 
33. LMAB Van Bortel, PMH Schiffers, ROB Böhm, IMV Mooij, KH Rahn, HẠ Struijker Boudier. Verapamil alters plasma human ANP levels in bypertensive patients. Proceedings 31 th Dutch FEDERA Meeting, 76, 1990

34. MJF Kool, LMAB Van Bortel, JAG Wijnen, APG Hoeks, HA] Struijker Boudier. Circadian rhythm and vessel wall properties of large arteries. Neth J Med 37 (4). A38, 1990

35. LMAB Van Bortel, PMH Schiffers, ROB Bohm, JMV Mooij, KH Rahn, HA] Struijker Boudier. Plasma ANP levels during hypertensive treatment with verapamil. Neth J Med 37 (4), A37, 1990

36. L Van Bortel, J Joosten, J Breed, J Kragten, F Lustermans, J Mooij. Quality of life with nebivolol. Neth J Med 37 (4), A36, 1990

37. LMAB Van Bortel, MA van Baak. Similar antihypertensive effect but different exercise endurance performance of two beta-blocking agents. Neth J Med 37 (4), A36, 1990

38. L Van Bortel, J Joosten, J Breed, J Kragten, F Lustermans, J Mooij. Quality of life assessed by the ISH questionnaire during antihypertensive treatment with nebivolol. Plrarm Wkbld Sci suppl C, C4, 1990

39. LMAB Van Bortel, T van Merode, FAM Smeets, ROB Böhm, RS Reneman. Verapamil and nebivolol improve carotid artery distensibility in hypertensive patients. Abstractbook 2nd International Congress Prospects and strategies in cardiovascular research, 1990

40. LMAB Van Bortel, MA van Baak. Exercise endurance performance during treatment with two beta-blocking agents. Eur J Pharmacol 183, 862, 1990

41. L Van Bortel, J Joosten, J Breed, J Kragten, F Lustermans, J Mooij. Quality of life with and antihypertensive effect of nebivolol. Eur J Pharmacol 183, 838, 1990

42. MJF Kool, JAG Wijnen, APG Hoxks, HAJ Struijker Boudier, LMAB Van Bartel. $V$ sassel wall propertic's and circadian rhythm in large arteries. Eur J Pharmacol $183,825,1990$

43. LMAB Van Bortel, T van Merode, FA Smeets, ROB Böhm, RS Reneman, HA] Struijker Boudier. Carotid artery distensibility and compliance in hypertensive patients during treatment with verapanil and nebivolol. Eur J Pharmacol 183 , 696,1990

44. LMAB Van Bortel, 'T van Merode, FAM Smeets, ROB Böhm, RS Reneman. Anthypertensive treatment and vessel wall properties of large arteries. Abstract book symposium "Pharmacology of vascular and cardiac remodelling", Maastricht, 1990 
45. JAG Wijnen, MJF Kool, H Kuipers, MA van Baak, HAJ Struijker Boudier, FTJ Verstappen, LMAB Van Bortel. Vessel wall properties of large arteries in trained and sedentary subjects. Abstract book symposium "Pharmacology of vascular and cardiac remodelling", Maastricht, 1990

46. L Van Bortel, $T$ van Merode, F Smeets, R Reneman, H Struijker Boudier. Nebivolol improves the vessel wall properties of the carotid altery in hypertensive patients. Abstracts 1st Int Nebivolol Investigators' meeting, Antwerp. September 13-16, $1990(\mathrm{~B} 7)$

47. LMAB Van Bortel, MA van Baak. Exercise tolerance with nebivolol and atenolol. Abstracts ist Int Nebivolol Investigators meeting, Antwerp, September $13-16,1990(\mathrm{C} 13)$

48. L. Van Bortcl, M Kool, J Wijnen, II Struijker Boudier. Beta-adrenoceptor blockade and beta-1 selectivity of nebivolol and atenolol. Abstracts 1st Ine Nebivolol Investigators' necting, Antwerp, Scptember 13-16, 1990 (C11)

49. LMAB Van Bortel, MA van Baak, MJF Kool, JAG Wijnen, HAJ Struijker Boudier. Beta-adrenoceptor blockade, antihypertensive effect and exercise tolerance with nebivolol and atenolol. Pharm Wkbld Sci 12 (suppl 6), J4, 1990

50. T Noordzij, J Wijnen, L Van Bortel, K Leunissen. Venous compliance is norma! in normotensive dialysis patients. Nephrol Dial Transplant 5, 708, 1990

51. T van Mcrode, PJJ Hick, FAM Smeets, ROB Böhm. LM Van Bortel, RS Reneman. Decreased carotid artery distensibility in borderline hy pertensive men - long-term follow-up. J Hypertens 8 (suppl 3), 302, 1940)

52. WH Birkenhäger, HAJ Struijker Boudier, LMAB Van Bortel, PAF Jansen, JH Bolk, A van der Kuy. De fysiologie van de oudere hypertensie-patient. Pharm Wkbld sci 125 (35), 908-911, 1990

53. LMAB Van Bortel, T van Merode, APG Hocks, RS Reneman, HAJ Struijker Boudier. Large arteries and hypertension. Acta Clin Belg 46(4), 261, 1991

54. LMAB Van Bortel, J Breed, J Kragten, F Lustermans, J Mooij, E Snoeck and A Van Peer. The use of population kinetics. Pharm Wkbld Sci 13 (suppl E), E4, 1001

55. LMAB Van Bortel, MIF Kool, JAG Wijnen, HAJ Struijker Boudier. Betaadrenoceptor blocking properties and antihypertensive effect differ between nebivolol and atenolol. Proceedings Internistendagen, 91, P174 (abstr nr 99), 1991

56. MIJF Kool, T van Merode, APG Hocks, LMAB Van Bontel. Reproducibility measurements with a new vessel wall movement detector system. Proceedings Internistendagen 91, P187 (absur nr 112), 1991 
57. MIF Kool. I XG Wijnen, H Kuipers, MA van Baak, HAI Struijker Boudier, LMAB Van Bortel. Do vessel wall properties of superficial arteries differ between endurance trained and sedentary subjects? Proceedings Intemistendagen 91 , Pliss (abstr nr 113), 1991

58. I.M. AB Van Bortel. MA van Baak. Nebivolol, a selective beta- I adrenoceptor antagonist, does not alter exercise tolerance. Cardiovase Drugs Ther 5 (suppl 3), P+23 (abstr ne P+99), 1991

59. LMA Van Bortel. Vasculaire veranderingen als basis voor een juiste keuze van het antihypertensivum. Proceedings Simpelveld Symposium "Behandeling van risikofaktoren zonder nieuwe risiko's te introduceren", 1991

60. LMAB Van Bortel, PMH Schiffers, IMV Mooij. Invasive haemodynamics of nebivolol in hypertensive patients. Drug Invest 3 (suppl 1), 60, 1991

6l. LMAB Van Bortel, J Joosten, J Breed, J Kragten, Fi Lustermans, I Mooij. Nebivolol in hypertensive patients. A placebo-controlled multicentre study. Drug Invest 3 (suppl 1), 105.106, 1991

62. LMAB Van Bortel, MJF Kool, JAG Wijnen, HAJ Struijker Boudier. Betaadrenoceptor blockade and beta-1 selectivity of nebivolol and atenolol. Drug Invest 3 (suppl 1), 173, 1991

63. LMAB Van Bortel, MA van Baak. Exercise tolerance with ncbivolol and atenolol. Drug Invest 3 (suppl 1), 176, 1991

64. MJF Kool, JAG Wijnen, LMAB Van Bortel, HAJ Struijker Boudier. Circadian pattern of vessel wall properties of large arteries in healthy men. Ricerca Sci Educ Perman suppl 90, abstr nr 345, 1991

65. MJF Kool, FHM Derkx, JAG Wijnen, HAJ Struijker Boudier, LMAB Van Bortel. Different diurnal variation in prorenin and renin. Ricerca Sci Educ Perman suppl 90, abstr nr 346, 1991

66. MJF Kool, T van Merode, APG Hoeks, LMAB Van Bortel. Reproducibility of a newly developed vessel wall movement detector system. Ricerca Sci Educ Perman suppl 90, abstr nr 347, 1991

67. JP Kooman, JAG Wijnen, P Draijer, LMAB Van Bortel, HAJ Struijker Boudier, JP van Hooff, KML Leunissen. The peripheral venous system in normotensive and hypertensive patients on hemodialysis (HD). J Hypertens 9 (suppl 6), 547, 1991

68. JAG Wijnen, MJF Kool, H Kuipers, MA van Baak, HAJ Struijker Boudier, LMAB Van Bortel. Differences in vessel wall properties of superficial arteries in cyclists and sedentary subjects. Ricerca Sci Educ Perman suppl 90, abstr nr 773, 1991 
69. JAG Wijnen, MJF Kool, LMAB Van Bortel, MA van Baak, HAJ Struijker Boudier. Excrcise training and ambulatory blood pressure in sedentary male subjects. Ricerca Sci Educ Perman suppl 90, abstr nr 774, 1991

70. L. Van Bortel, T van Merode, F Smeets, R Reneman, H Struijker Boudier. Effect of antihypertensive treatment on the vessel wall properties of large arteries. Arch Int Pharmacodyn Ther 310, 190, 1991

71. JAG Wijnen, MJF Kool, MA van Baak, HAJ Struijker Boudier, LMAB Van Bortel. Exercise training does not lower ambulatory blood pressure in sedentary male subjects. Eur Heart J 12 (suppl), 29 (abstr nr 260), 1991

72. MJF Kool, FHM Derkx, JAG Wijnen, HAJ Struijker Boudier, L.MAB Van Bortel. Prorenin and renin do not have the same diurnal variation. Eur Heart J 12 (suppl), 151 (abstr nr 857), 1991

73. LMAB Van Bortel, MJF Kool, FHM Derkx, JAG Wijnen, HAJ Struijker Boudier. Diurnal variation in humoral factors. Pharm Wkbld Sci 13 (suppl L), L5, 1991

74. MJF Kool, JAG Wijnen, HAJ Struijker Boudier, L.MAB Van Bortel. Vessel wall properties of large arteries show a diurnal variation. Pharm W'kbld Sci 13 (suppl L). $[.5,1991$

75. All IM Houben, JAG Wijnen, LMAB Van Bortel, AC Nieuwenhuyzen Kruseman. Local hyperglycemia increases regional blood flow directly in man. Diabetes 4 (suppl 1), 212A, 1991

76. AJHM Houben, FHW Jungbauer, JAG Wijnen, LMAB Van Bortel, DW Slaaf, AC Nieuwenhuyzen Kruseman, NC Schaper. Effects of local hyperglycaemia on forearm blood flow. Int J Microcirc 10, 379, 1991

77. JP Kooman, P Draaijer, JAG Wijnen, LMAB Van Bortel, HAJ Struijker Boudier, JP van Hoofl, KMI. Leunissen. Functional reactivity of the peripheral venous system in patients on hemodialysis. Blood Purif $9(1), 35,1991$

78. JP Kooman, U Gladziwa, G Böcker, JAG Wijnen, L Van Bortel, JP van Hooff, KML Leunissen. Non-invasive blood pressure measurements during the interdialytic period in hemodialysis in patients. JASN 2(3), 334, 1991

79. JP Kooman, U Gladziwa, JAG Wijnen, G Böcker, L. Van Bortel, HG Peltenburg, IP van Hooff, KML. Leunissen. Representative blood pressure in hemodialysis patients by 48-hour ambulatory BP monitoring. Nephrol Dial Transpl $6(10)$, 803, 1991

80. IP Kooman, JAG Wị̣nen, TC Noordzij, L Van Bortel, JP van Hooff, KML Leunissen. Hypertension and not chronic renal failure is reducing peripheral venous compliance (PVC) in hemodialysis patients. Kidney Int 40,347, 1991 
81. LMAB Van Bortel. MA van Baak, MJF Kool. JAG Winnen. HAT Struijker Boudier. Beta-adrenoceptor blockade and exercise tolerance. Arch hu i1 1, 191-192, 1991

82. P Draaijer, M Kool, L Van Bortel, K Leunissen, L. Henderson. Arterial distensibility (DC) and compliance (CC) in salt-resistant and salt-sensitive borderline hypertensive patients (SRBHT vs. SSBHT). JASN 2, 474, 1991

83. HAJ Struijker Boudier, MJF Kol, JAG Wijnen, LMAB Van Bortel. Circadian changes and effect of exercise training, Abstract book workshop on "Func" tional and structural properties of large arteries", Paris, 1992

84. LMAB Van Bortel, APG Hoeks, MJF Kool, IIAJ Struijker Boudier. Large artery properties as a target for risk reduction by ancilyypertensive therapy. Abstract book workshop on "Functional and structural properties of large arteries", Paris, 1992

85. LMAB Van Bortel, MJF Kool, JGS Breed, J De Crée, FATh Lustermans, HHW Thijssen. Hemodynamic effects of nebivolol and atenolol. Naunyn Schmiedeberg's Arch Pharmacol 345 (suppl), 63, 1992

86. MJF Kool, APG Hoeks, HAJ Struijker Boudier, LMAB Van Bortel. Vessel wall properties in habitual smokers. Neth I Med 40, A58, 1992

87. MJF Kool, APG Hoeks, HAJ Struijker Boudier, LMAB Van Bortel. Acute effect of smoking on large artery compliance. Neth J Med 40: A59, 1992

88. MJF Kool, APG Hoeks, HAJ Struijker Boudier, LMAB Van Bortel. Effects of smoking on large artery compliance. Pharm Wkbld Sci (suppl D), D4, 1992

89. P Draajer, M Kool, L. Van Bortel, K Leunissen, P de Leeuw. Arterial distensibility and compliance in salt-resistant and salt-sensitive borderline hypertensive patients. J Hypertens 10 (suppl 4), \$86, 1992

90. LMAB Van Bortel, JA Wijnen, MJ Kool, MA van Baak, HAJ Struijker Boudier. Effect of exercise training on ambulatory blood pressure. ) Hypertens 10 (suppl 4), $\$ 106,1992$

91. L, Van Bortel, J Breed, J Kragten, F Lustermans, J Mooij, E Snoeck, A Van Peer. Population kinetics applied to steady-state plasma levels from a clinical trial. Abstracts Vth World Conference on Clinical Pharmacology and Therapeutics, O-37-05, 161, 1992

92. LMAB Van Bortel, MJF Kool, T van Merode, APG Hoeks, RS Reneman, HAJ Struijker Boudier. Non-invasive measurements of arterial compliance: reproducibility. Abstracts Vth World Conference on Clinical Pharmacology and Therapeutics, P-404-03, 263, 1992 
93. LMAB Van Bortel, MJF Kool, JAG Wijnen, HAJ Struijker Boudier. Nebivolol: not only a beta-1 selective adrenoceptor antagonist. Abstracts Vth World Conference on Clinical Pharmacology and Therapeutics, P-404-03, 1992

94. LMAB Van Bortel, MJF Kool, JGS Breed, FATh Lustermans, JMV Mooij, J De Crée. In contrast to atenolol, nebivolol does not increase systemic vascular resistance. Eur Heart J 13 (abstract suppl), 21, 1992 (abstr nir 240)

95. LMAB Van Bortel, MJF Kool, FHM Derkx, JAG Wijnen, HAJ Struijker Boudier. Diumal variation in hemodynamics and humoral factors. Arch Int Pharmacodyn Ther $315,127,1992$

96. JP Kooman, U Gladziwa, G Böker, LMAB Van Bortel, JP van Hooff, KML leunissen. Role of venous compliance (VC) in hemodynamics during ultrafiltration. Kidncy Int 41, 1434, 1992

97. P Draaijer, M Kool, L Van Bortel. JP van Hooff, KML Leunissen. Arterial distensibility (DC) and compliance (CC) in salt-resistant and salt-sensitive borderline hypertensive patients (SRBHT vs. SSBHT). Kidney Int 41, 1433, 1992

98. MA van Baak, JAG Wijnen, C de Haan, H Struijker Boudier, L Van Bortel. Beta-blockade and lipolysis during enclurance exercise. Med Sci Sports Exercise 24 (suppl 5), 187, 1992

9). P' Dratijer, M Kool, I, Van Bortel, P de Leeuw, K Leunissen. Arterial distensibility (DC) and compliance (CC) in saltresistant and salt-sensitive borderline hypertensive pattents (SRBHT vs. SSBHT). Am J Hypertens 5, 36A, 1992

100. J Vollebregt, I. Van Bortel, Th de Vries, H van Rees. Ontwikkeling en toetsing van een meetinstrument voor het bepalen van de farmacotherapeutische vaardigheden van de basisarts. Abstractbook "Gezond Onderwijscongres", 1992

101. AJ luik, U Gladziwa, JP Kooman, JP van Hooff, PW de Leeuw, LMAB Van Bortel, KML Leunissen. Blood pressure rise $(\Delta R R)$ in relation to the length of the interdialytic period (IDP). JASN 3, 377, 1992

102. L. Van Bortel, J Breed R Ciampricotti, G Tromp, F Valster, F Lustermans, E Lageweg. Quality of life during antihypertensive treatment. A comparative study between bisoprolol and enlalapril. Pharm Wkbl Sci 14 (suppl J), J3, 1992

103. LMAB Van Bortel, MJF Kool, FAT Lustermans, JGS Breed, AGP Hoeks, HAJ Struịker Boudier. Does antihypertensive treatment with an ACE-inhibitor and a diuretic result in different effects on hacmodynamics and vessel wall properties of large arteries? Pharmacy World \& Sci 1993, 15 (suppl E):E4 
104. MJF Kool, J Lambert, AJM Donker, HAI Struijker Boudier, LMAB Van Bortel. Large artery compliance in young insulin-dependent diabetes mellitus. Ricerca Scientifica ed Educazione. Permanente suppl 95, 366, 1993

105. LM Van Bortel, JA Wijnen, MJ Kool, JP Kooman, MA van Baak, HA Struijker Boudier. Vessel wall properties of large arteries and endurance training. Ricerca Scientifica ed Educazione Permanente suppl 95, 784, 1993

106. MJF Kool, FAT Lustermans, JGS Breed, HAJ Struijker Boudier, APG Hoeks, L.MAB Van Bortel. Effect of perindopril and amiloride/hydrochlorothiazide on haemodynamics and vessel wall properties of large arteries. Ricerca Scientifica ed Educazione Permanente suppl 95, 367, 1993

107. M Kool, F Lustermans, J Breed, H Struijker Boudier, A Hocks, L Van Bortel. Effect of Coversy $\left.\right|^{R}$ and Moduretic mitis ${ }^{R}$ on blood pressure, cardiac function and vesscl wall properties of large arteries. Eur Heart J 14, 65 (P527), 1993

108. M Kool, A Hoeks, H Struijker Boudier, R Reneman, L Van Bortel. Are the acute effects of smoking on arterial wall properties the trigger for acute cardiovascular events in habitual smokers? Eur Hearı J 14, 65 (P1984), 1993 
